\title{
Convergence of biological and psychological perspectives on cognitive coordination in schizophrenia
}

\author{
William A. Phillips \\ Department of Psychology, Center for Cognitive and Computational \\ Neuroscience, University of Stirling, Stirling FK9 4LA, Scotland, \\ United Kingdom. \\ wap1@stir.ac.uk
}

\section{Steven M. Silverstein}

Department of Psychiatry, Psychotic Disorders Division, Weill Medical College of Cornell University, New York Presbyterian Hospital, White Plains, NY 10605

steven.silverstein@att.net

\begin{abstract}
The concept of locally specialized functions dominates research on higher brain function and its disorders. Locally specialized functions must be complemented by processes that coordinate those functions, however, and impairment of coordinating processes may be central to some psychotic conditions. Evidence for processes that coordinate activity is provided by neurobiological and psychological studies of contextual disambiguation and dynamic grouping. Mechanisms by which this important class of cognitive functions could be achieved include those long-range connections within and between cortical regions that activate synaptic channels via NMDAreceptors, and which control gain through their voltage-dependent mode of operation. An impairment of these mechanisms is central to PCP-psychosis, and the cognitive capabilities that they could provide are impaired in some forms of schizophrenia. We conclude that impaired cognitive coordination due to reduced ion flow through NMDA-channels is involved in schizophrenia, and we suggest that it may also be involved in other disorders. This perspective suggests several ways in which further research could enhance our understanding of cognitive coordination, its neural basis, and its relevance to psychopathology.
\end{abstract}

Keywords: attention; cerebral cortex; cognitive coordination; cognitive neuropsychiatry; cognitive neuropsychology; context disorganization; Gamma rhythms; Gestalt theory; glutamate; grouping; memory; NMDA-receptors; PCP-psychosis; perceptual organization; schizophrenia

\section{Introduction}

Cognitive neuroscience provides a new conceptual framework for psychiatry by showing how psychological processes arise from neuronal activity (Kandel 1998). Conversely, it is possible that psychiatry will influence the future development of cognitive neuroscience by encouraging a better balance between localist and holistic conceptions of brain function. An apparent conflict between these conceptions has been central to the development of neuroscience, with the emphasis upon locally specialized functions emerging as clearly dominant. Neuroanatomy, neurophysiology, neuropsychology, and neuroimaging all show that different regions of the brain process information about different things, and that different cells within regions deal with different aspects of those things. Recent developments in experimental and theoretical neurobiology, however, are leading to an increased emphasis upon interactions that coordinate the activity of locally specialized processors. Here we argue that impairment of these coor-
William A. Phillips, Ph.D., is Professor of Neuropsychology at the University of Stirling, Scotland UK. He has published more than 70 papers on vision, visual memory, perceptual learning, childrens drawings, the effects of brain damage on reading and writing, and the theory of neuronal computation. He was a founder and the first Director of the Center for Cognitive and Computational Neuroscience at the University of Stirling.

Steven M. Silverstein, Ph.D., is Associate Professor of Psychology in Psychiatry at the Weill Medical College of Cornell University, in White Plains, NY, USA. He has published approximately 50 papers in the areas of schizophrenia and cognition. These include studies of information processing deficits in schizophrenia and other neurologic and developmental disorders, as well as demonstrations of treatment approaches to rehabilitate these impairments. 
dinating interactions may be central to some psychiatric disorders. Studies of such disorders may therefore shed further light on their nature and importance.

Many studies indicate that the strength and salience of neuronal responses depends on context, and that subsets of neuronal responses are grouped by synchronizing the spiking activity of which they are composed (Gray 1999; Phillips \& Singer 1997a). This implies that context can change the salience and timing of neuronal signals but without changing what they mean. These effects are a special kind of modulation, which we will refer to as cognitive coordination. Such effects were predicted by theoretical considerations (e.g., Edelman 1989; Sporns et al. 1989; von der Malsburg \& Schneider 1986), and Phillips and Singer (1997a) combine the experimental evidence with neurocomputational theory to argue that it is fundamental to normal cortical function. Many others agree with this view (Phillips \& Singer 1997b), and the possibility that contextual coordination may be impaired in schizophrenia was briefly noted by Silverstein and Schenkel (1997). This paper examines that possibility in detail. The viewpoint presented is consistent with the theory relating schizophrenia to re-entrant mechanisms (Edelman 1989; Tononi \& Edelman 2000), and with the disconnection hypothesis (Dolan et al. 1999; Friston 1999), with which it will be compared in section 7.1.

Changes in the effects of context are central to several influential theories of cognition in schizophrenia (e.g., Cohen et al. 1999a; Cohen \& Servan-Schreiber 1992; Gray et al. 1991; Hemsley et al. 1993; Shakow 1962). They are not central to a currently influential conception of the underlying pathophysiology (e.g., Olney et al. 1999), however. This hypothesizes that under-activity of NMDA (N-methyl-Daspartate) glutamate receptor channels plays a central role, and it is supported by a rapidly growing body of neuropharmacological studies cited below. Here we argue that these two streams of research are mutually supportive.

Many patients diagnosed with schizophrenia have impairments in perception, pre-attentive sensory gating, selective attention, working memory, and long-term memory, as well as other cognitive impairments more obviously interpreted in terms of context. We will argue that all these impairments involve cognitive coordination. As our theory predicts, they are most prominent in patients with thought disorder and other disorganized symptoms, so those are the symptoms on which we focus. Liddle (1987) reported evidence for three distinct groups of symptoms in schizophrenia: reality distortion (hallucinations and delusions), negative symptoms, and cognitive disorganization. Though subsequent factor analytic studies sometimes also suggest one or two additional factors, they reliably find evidence for these three (Andreasen et al. 1995). To a crude first approximation they can be seen as relating to Kraepelin's classic distinctions between paranoia, catatonia or negativism, and hebrephrenia, with the crucial proviso that they provide a 3-dimensional space that can be used to characterize some of the variation in schizophrenic symptomatology, without at all implying that patients are clustered within one of three separate regions of that space.

After 100 years of research on schizophrenia, it is still unclear whether schizophrenia is one disorder or several. The presenting symptoms are clearly heterogeneous, and at the neurobiological level multiple neurotransmitters and brain regions have been implicated (e.g., Benes 2000b; Jentsch \& Roth 1999). Recognizing this heterogeneity, we must make clear at the outset that we are here primarily concerned with the disorganization syndrome, and with the disorders of coordinating neuronal interactions, such as under-activity of NMDA-receptor channels, from which disorganization may arise.

It is sometimes suggested that much of the observed heterogeneity arises in various ways from a common underlying pathology. We will argue that the disorganization syndrome, which has a particularly large genetic loading (Cardno et al. 2001), may reflect such a pathology.

Nevertheless, we do not claim that this can explain all symptoms that have been associated with schizophrenia, and assume that some can arise independently of either cognitive disorganization or NMDA-hypofunction. Similarly, we do not attempt to explain all of the many neuropathological findings relating to schizophrenia, but focus on those that seem particularly relevant to our hypothesis.

We will discuss four crucial issues concerning cognitive impairments. First, what is "context"? Second, do changes in context-sensitivity underlie various apparently diverse cognitive impairments? Third, how are cognitive impairments related to clinical symptoms? Fourth, are these cognitive changes similar to those produced by substances such as PCP (phencyclidine) and ketamine, and, if so, what does that suggest about the pathophysiology of the underlying cognitive changes?

Dopamine dysregulation has been implicated in schizophrenia by a vast amount of research, but we will outline evidence suggesting that there is also a central role for underactivity of NMDA glutamate receptor channels. For brevity we will refer to this as NMDA-hypoactivity.

We will discuss three issues raised by this evidence. First, why does NMDA-hypoactivity have such widespread but distinctive effects upon cognition? Second, what is the role of NMDA-mediated interactions between pyramidal cells in producing these effects? Third, the most distinctive property of NMDA-channels is that, in addition to being ionotropic, they are voltage-dependent. Theories of cortical computation claiming that NMDA-channels play a major role in coordinating activity (e.g. Phillips \& Singer 1997a; Sporns et al. 1989) emphasize this property, and thus predict that NMDAhypoactivity will impair cognitive coordination.

We will cite previous work clarifying the key notions of "context" and "coordination" from psychological, neurobiological, and computational perspectives, but we see these concepts as open to further improvement. We will focus on coordination within the cortex but assume that it is also relevant to limbic, striatal, and thalamic functions. Although context has long been thought relevant to schizophrenia (e.g. Shakow 1962), different investigators think of it in different ways (Pickering 1993). Cohen and Servan-Schreiber (1992) identify context with task-relevant information supplied by preceding events and stored in a working memory (WM) involving the prefrontal cortex (PFC). For others (e.g., Gray et al. 1991; Hemsley et al. 1993; Jones et al. 1991) it includes effects of concurrent context on perception, and depends more upon long-term memory than upon working memory (WM). Our concept of context includes both of these views. It is based upon an underlying distinction between processes that determine what neural signals mean, that is, what they transmit information about, and processes that affect transmission of those signals without becoming part of their meaning. Effects upon salience or timing provide good examples because it is assumed that 
they do not change the meaning of the signals affected. We will call the input about which a processor transmits information to subsequent processors the "primary input." It arises from the receptive field (RF) input determining the stimuli to which cells are selectively sensitive. Context arising from other streams of processing plays a secondary role by modifying signal detection decisions, by influencing the choice between possible interpretations, by making relevant signals more salient, and by grouping those that go together. The input producing these effects has therefore been called the contextual field input (Phillips \& Singer 1997a). We do not identify context with particular kinds of knowledge, but with a particular class of effects, assuming that the outputs of most processors serve both as a basis for computing higher-order things and as a context for the processing of other things. Although we discuss the distinction between contextual interactions and the primary interactions that determine RF selectivity as though it were categorical, the biological reality may well be less simple, combining these two functions in various ways. ${ }^{1}$ Even if this is so, however, it remains necessary to make the concept of contextual interaction clear. Formal computational studies of our conception of context will be cited below. Here we try to convey the essentials through informal demonstrations.

Information can be ambiguous in several ways as the presence, interpretation, and relevance of signals can all be uncertain given just those signals alone. Figure 1 shows examples of disambiguation by concurrent stimulus context, making explicit the properties that distinguish the effects of context from the effects of primary input. Figure 1 emphasizes the effects of concurrent stimulus context, and deficits in such effects will be emphasized below. Task contexts are also important, however. Many paradigms have been used to study the effects of task-context in schizophrenia, and these effects are also usually much reduced (e.g., Perlstein et al. 1998).

Local stimulus elements are related to the context in which they occur by being grouped into coherent wholes or Gestalts. Evidence reviewed in section 4.1 shows that these basic organizational processes are often impaired in schizophrenia. The Gestalt theory-influenced psychiatrists Matussek (1952/1987) and Conrad (1958) saw core components of schizophrenia as arising from a disorder of perceptual organization, and Cutting (1989) concluded that Gestalt theory offers an understanding of otherwise inexplicable phenomena of schizophrenia. Here, we further develop these hypotheses by reviewing evidence that, as the Gestalt psychologists emphasized, such dynamic organizational processes may apply to cognition in general.

In summary, our working conceptions of coordination and context are as follows. Coordinating interactions are those that affect the salience or dynamic grouping of neuronal signals without changing what they mean. Such interactions do affect the interpretation of stimulus inputs, however. This is shown in Figure 1 where the interpretation of stimulus items is strongly influenced by surrounding items. We assume that this involves increasing the salience of the neuronal signals that convey the interpretation selected, which implies that those signals can be made more salient, without changing what they mean. Dynamic grouping creates new combinations of items while maintaining their distinct meanings. It is essentially the same as "compositionality" (Fodor \& Pylyshyn 1988), which refers to the ability to compose new structures, such as sentences, from elements,

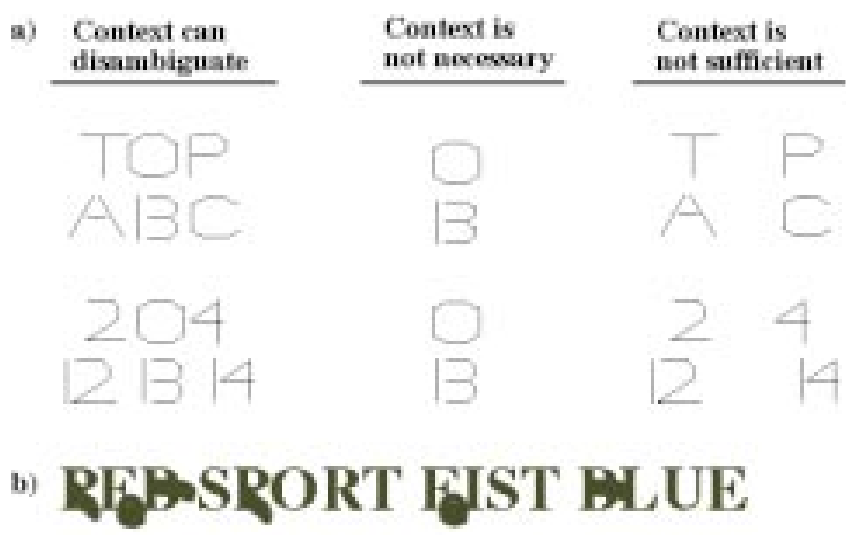

Figure 1. Simple examples showing the distinct roles of primary and contextual input, in the case of disambiguation by concurrent stimulus context. (a) Left column: nearby items affect the salience of the various possible interpretations of the central item. Though $\mathrm{T}$ and $\mathrm{P}$ influence the processing of $\mathrm{O}$, a response identifying it as a letter rather than a digit does not imply that it is flanked by $\mathrm{T}$ and $\mathrm{P}$, or indeed by any context at all. Thus the response transmits information about the primary input, and not about the context, even though it is affected by the context. Center column: context is not necessary as the central item is perceived in the absence of any context, though interpretation may then either select a dominant default option or fluctuate between alternatives. Right column: Primary input is necessary, however, as contextual items alone do not produce perception of items for which there is no primary evidence. (b) Mutual contextual disambiguation can occur when all items are ambiguous.

such as words, which retain their individual meanings while being used in many different combinations. Fodor and Pylyshyn (1988) argued that standard connectionist models fail to provide this capability and are therefore of limited value in modelling higher aspects of cognition. The coordinating interactions emphasized here could overcome this limitation because forming new combinations of elements while retaining their individual meanings is one of their central functions.

It may seem that localist conceptions of brain function have long since won the debate with holistic conceptions by explaining coordination in terms of a central executive located in specialized regions of the pre-frontal cortex (PFC). This cannot be the whole story, however. Organizing activity by imposing top-down strategic commands upon what would otherwise be anarchy could not by itself be adequate, because any strategic control system must be ignorant of nearly all of the details upon which effective local cooperation depends. In relation to perception it is now clear that the dynamic organizational processes emphasized by Gestalt psychology are largely pre-attentive (Watt \& Phillips 2000). In relation to motor control, the longestablished principle of "central executive ignorance" implies that dynamic coordination is largely local (Kelso 1995; Turvey 1977). Our working hypothesis is therefore that cortical activity is coordinated by widely distributed local interactions within and between regions, as well as by topdown strategic commands, and we will outline evidence that this is so.

These arguments imply that there are higher-level processes of strategic coordination. ${ }^{2}$ Our working hypothesis is that local and strategic coordination both depend on basic 
physiological mechanisms of dynamic grouping and contextual-modulation. In the following section we discuss the relevance of these mechanisms to pre-attentive perception. Here we briefly note their possible relevance to executive functions. The control of lower-level routines clearly requires modulatory interactions, as does attention. Working memory requires forming and maintaining a novel sub-set of items, which implies some form of dynamic grouping (Lisman et al. 1998; Luck \& Vogel 1997; Raffone \& Wolters 2001). Source memory and the assignment of affective significance both imply that contextual associations are formed from semantically distinct items, thus requiring compositionality. Lastly, although it is not known what neuronal processes are involved in creative problem-solving, they seem likely to include both dynamic organization and context-sensitivity.

What we call local coordination has much in common with "contention scheduling" (Shallice 1988; Shallice \& Burgess 1996), that is, with the automated resolution of conflicts between schemata. Our emphasis upon local coordination does not imply that we think executive functions have little to do. On the contrary, there is evidence that, in addition to their other roles, executive functions can modulate local processes of dynamic coordination (Fries et al. 2001; Gilbert et al. 2000; Ito \& Gilbert 1999). ${ }^{3}$

Our perspective on context and coordination has been presented here informally, but it has been given rigorous mathematical specification using Shannon's information theory (Phillips et al. 1995; Tononi et al. 1994; 1996), and it has been implemented in simulations of simplifying (Kay et al. 1998) and physiologically realistic (Sporns et al. 1989) neural networks. Introductory nonmathematical reviews of this work that discuss its relevance to cognition are available (Phillips \& Singer 1997a; Tononi et al. 1998b). Computational theories provide ways of bridging the gap between psychiatry and neurobiology (e.g., Cohen \& ServanSchreiber 1992; Hoffman \& McGlashan 1993). An explicit bridge is necessary, because we will never know whether an identified neural pathology is directly responsible for the cognitive impairments with which it is found to be associated unless we know how the mechanism that is impaired gives rise to the cognitive capacities concerned.

The central concern of this target article is therefore with the neural bases of cognitive disorganization in schizophrenia. Section 3 reviews evidence implicating NMDAhypofunction in this disorder. Section 4 reviews evidence that it involves deficits in basic processes of cognitive coordination, such as contextual disambiguation and dynamic Gestalt organization. We claim that the neurocomputational theories of Kay et al. (1998), Phillips et al. (1995), and Tononi et al. (1994) can explain why NMDA-hypofunction should lead to these particular cognitive deficits. Section 2 , therefore, first reviews psychophysical, anatomical, and physiological evidence for the central ideas expressed in those theories. The computational work simply shows that the operations involved are formally coherent and able to perform the functions involved. Empirical studies are necessary to determine whether they are relevant to neurobiology. Section 5 reviews studies of high-frequency cortical rhythms that provide further support for the view that cognitive coordination, NMDA-activity, and psychosis are all related. Section 6 shows how our perspective is compatible with the heterogeneity of symptoms in schizophrenia, and also briefly discusses the possibility that impaired cognitive coordination may be involved in some other disorders of mental function, such as autism. Section 7 compares our perspective with related theories. Section 8 lists several of the issues that arise, and which in our opinion deserve closer examination.

\section{A special class of neuronal interactions coordinate cortical activity}

\subsection{There is physiological and psychophysical evidence for coordinating interactions}

There is good evidence that both stimulus and task contexts affect activity by enhancing relevant and suppressing irrelevant activity. For example, parallel studies comparing results obtained from human psychophysics and single-unit recording in the visual cortex of alert monkeys show that they are quantitatively very similar (Kapadia et al. 1995). Here we outline this evidence, which has been thoroughly reviewed by others (Desimone \& Duncan 1995; Gilbert 1992; Kovács 1996; Lamme \& Spekreijse 2000; Maunsell 1995; Salin \& Bullier 1995; Singer \& Gray 1995; Wörgötter \& Eysel 2000; Zipser et al. 1996). Context influences the salience of neuronal responses. Pyramidal cells in the primary visual cortex, for example, are specialized to respond preferentially to stimuli with particular positions, orientation and size. This selectivity is determined by the primary input to each cell, which arises from a limited region of the visual field which is called the receptive field $(\mathrm{RF})$ of that cell. Responses to preferred stimuli within the RF can be amplified or suppressed by stimuli presented well beyond the classical RF, however. This interaction is highly specific to the geometric relations between elements, with facilitation being strong when the local elements taken together form some coherent entity such as a smooth contour (Kapadia et al. 1995; Kovács 1996). Facilitation is strongest when the RF input is weak, and decreases as the spacing between elements increases (Polat et al. 1993; Polat \& Sagi 1998). Locally specific contextual amplification in primary visual cortex has also been shown to be produced by feedback signals from extrastriate cortex that increases the salience of stimulus elements that are part of the figure rather than the ground (Zipser et al. 1996). Though in a sense "top-down," these particular effects are nevertheless still driven by stimulus context, which is used automatically to organize input into figure and ground (Zipser et al. 1996). Similar effects on salience can also be produced by processes of selective attention that depend upon the task (Desimone \& Duncan 1995; Maunsell 1995). These effects of task context are produced by the same kind of physiological mechanisms as those that mediate the effects of stimulus context (Treue \& Tujillo 1999). Furthermore, task context modulates the effects of local stimulus context (Gilbert et al. 2000; Ito \& Gilbert 1999).

Contextual interactions are also involved in the grouping of neuronal responses. The conditions in which simple visual elements, such as Gabor patches, are grouped, are so similar to those under which disambiguating interactions occur between them, that both effects could depend upon a common set of underlying neuronal connections (Kovács 1996). Dynamic Gestalt grouping has a fundamental role in vision, and is predominantly computed by pre-attentive mechanisms. One particularly clear example of a task in which dynamic grouping is necessary is the computation of 
coherent object motion from many local motion signals (Watt \& Phillips 2000), and relevant studies of motion perception in schizophrenia will be discussed in section 4.1.

Dynamic grouping could be signalled by synchronizing the activity to be grouped, and this hypothesis is supported by a wide variety of evidence (Gray 1999; Singer 1994; 1995; 1999; Singer \& Gray 1995). Synchrony was first observed in primary visual cortex of anaesthetised cats, and it has since been shown in awake monkeys (Kreiter \& Singer 1996), and within and between parietal and motor cortex (Roelfsema et al. 1997). Psychophysical evidence on the role of dynamic grouping in vision and its relation to synchrony is reviewed by Watt and Phillips (2000). Evidence from evoked potentials indicates that synchrony is enhanced by selective attention in auditory cortex (Tiitinen et al. 1993), and it has been argued that synchrony may be the neural correlate of sensory awareness (Engel \& Singer 2001). Though the role of synchrony remains controversial, Treisman (1999) concludes that there is so much evidence for grouping through convergence in pre-specified hierarchies and for grouping as signalled by synchronization that it is highly likely that both occur. Watt and Phillips (2000) argue that they are mutually supportive.

\subsection{Lateral and descending intracortical connections provide an anatomical basis for coordination}

Long-range communication within and between cortical regions is mediated predominantly by pyramidal cell axons (Braitenberg \& Schüz 1991). Though complex, this longrange communication includes some simple patterns of connectivity that are common to cortex as a whole (Felleman \& Van Essen 1991). Ascending feedforward connections provide the primary driving inputs that are processed by local cortical circuits. Long-range lateral connections within and between regions together with descending feedback connections provide additional input (Felleman \& Van Essen 1991), and this could in part be used to coordinate the primary feedforward processing. This general pattern of connectivity is shown as simply as possible in Figure 2a. Figure $2 \mathrm{~b}$ uses the canonical circuit hypothesized by Douglas and Martin (1990) to show how local circuits within cortical columns could receive both primary ascending inputs and long-range coordinating inputs. Figure 2 focuses on long-range connections omitting many details of intrinsic connectivity within the local circuits, such as the NMDAreceptor component of the local excitatory input to the inhibitory cells (Grunze et al. 1996). The long-range lateral connections within primary visual cortex have been studied in great detail. They are highly specific, and probably implement the coordinating interactions producing Gestalt organization (Gilbert 1992; Kovács 1996; Schmidt et al. 1997; Singer \& Gray 1995).

\subsection{NMDA glutamate receptor channels provide a synaptic mechanism for coordination}

The cholinergic, adrenergic, dopaminergic, and serotonergic systems are most prominent amongst neurotransmitters known to have modulatory effects. It is unlikely that these classical neuromodulators can implement the detailed coordinating interactions emphasized here, however. This is because those interactions must be between the processing streams that convey detailed information about what is be- a) Coordination within and between cortical regions

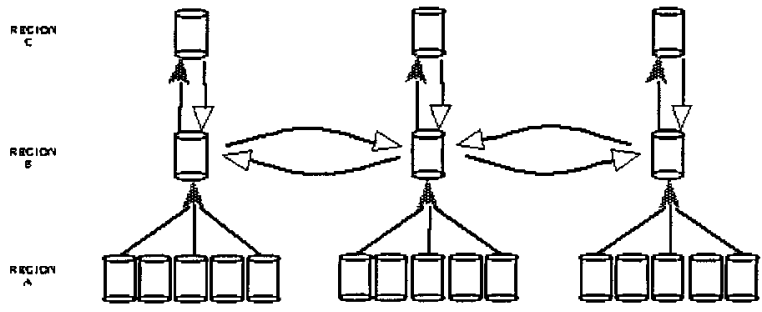

b) Connectivity within and between cortical columns

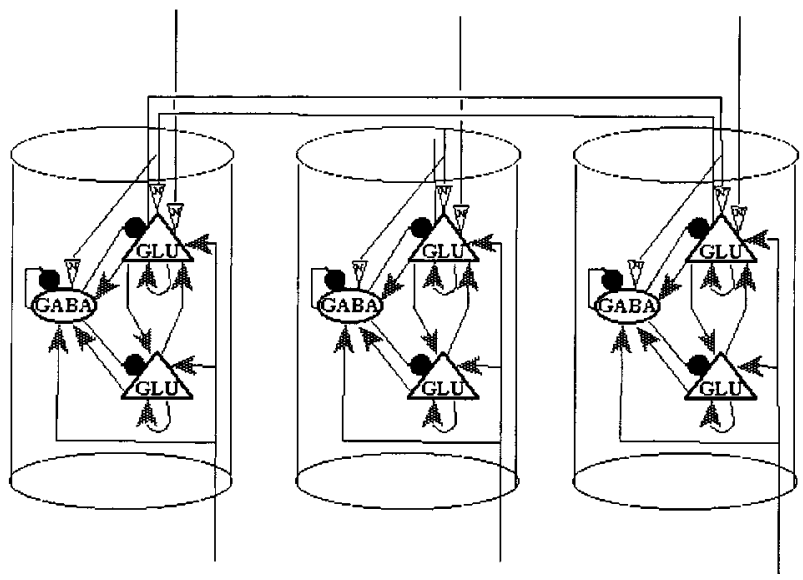

Figure 2. Common patterns of connectivity within and between cortical regions. (a) This shows the hypothesized distinction between RF connections that transmit primary feedforward information through cortical columns, shown with solid grey arrowheads and contextual field connections that coordinate that transmission, shown with open arrowheads. Only a few columns are shown in each region, and many complexities of connectivity are omitted. Each column of necessity receives input from only a small subset of the cells in the preceding region, and though these subsets may overlap for nearby columns (not shown) there is little overlap between the inputs of most columns within a region. Massively parallel but interactive processing is thus implied by this architecture. (b) Connectivity within and between columns is shown using the canonical cortical circuit hypothesized by Douglas and Martin (1990). Three columns processing separate parts of the input from the preceding region are shown. This pattern of connectivity is thought to occur throughout the cortex. Glutamatergic (GLU) pyramidal cells are divided into two populations with the upper group being those in Layers II, III, and IV and the lower being those in Layers V and VI. Excitatory synapses are shown by arrowheads. Inhibitory synapses formed by inhibitory cells are shown by filled circles. Synapses mediating long-range coordination within and between regions are shown by open arrowheads labelled N. They selectively link columns sensitive to distinct but correlated inputs, bypassing intervening columns that respond to inputs that are less correlated. The circuitry shown is highly simplified and omits much detail, including the pyramidal cell outputs to more distant sites, and the sources of the descending coordinating inputs from higher regions. For detailed reviews of evidence on which this figure is based, see Douglas and Martin (1990), Felleman and Van Essen (1991), and Salin and Bullier (1995). 
ing seen, heard, thought, or remembered, and so on. Consider the perception of the ambiguous stimuli in Figure 1, for example. Coherent interpretations at each level of processing are formed by coordinating the interpretation of each element with that of other elements. The information concerning these elements and their interpretation is transmitted by the axons of pyramidal cells, which are all glutamatergic (Braitenberg \& Schüz 1991). We therefore suggest that two major components of the glutamatergic system need to be distinguished: The first provides the primary input that produces post-synaptic output and determines what cells transmit information about. The second is neither necessary nor sufficient to produce post-synaptic output, but modifies the effects of the primary inputs. These modulatory interactions between pyramidal cells can be direct, but will often be mediated by local circuit neurons, as in the case of suppressive interactions for example, which depend on inhibitory interneurons. This distinction between primary and coordinating interactions is closely analogous to that between obligatory and modulatory interactions between cortical regions as inferred from fMRI (functional magnetic resonance imaging) data (Friston et al. 1995).

This argument is supported by a well-established distinction between two classes of glutamate receptor, one that produces primary excitatory drive and one that modulates the activity thereby produced. For a thorough introduction to these receptors see Feldman et al. (1997). AMPA and kainate glutamate receptors provide primary excitatory drive because they open whenever glutamate (GLU) binds to them. Metabotropic and NMDA-receptors both have a coordinating role, because both modulate the effects of direct excitatory inputs (Daw et al. 1993; Salt \& Eaton 1996). Though there may be a role for metabotropic receptors (Whittington et al. 1995), coordination on a fast time-scale is likely to be more dependent on NMDA-receptors because they operate more rapidly (Daw et al. 1993). This argument is made precise by computational studies showing how the voltage-dependent properties of NMDA-receptors can be used for the dynamic organization of both processing and learning (e.g., Kay et al. 1998; Phillips et al. 1995; Sporns et al. 1989). The argument is further strengthened by the evidence cited below, implicating NMDA-receptors in cognitive coordination.

As NMDA-receptors may play a crucial role in coordination we now note their most relevant characteristics: (1) They are voltage-dependent because at or below resting levels of post-synaptic membrane potential, they are blocked by magnesium ions (Fig. 3). Current flow through NMDA-channels thus requires both that glutamate binds to the receptor and that the post-synaptic membrane is already partially depolarized. The key point to note is that because of this voltage-dependency they could contribute to coordination by amplifying activity that is appropriate, and by suppressing, via inhibitory interneurons (Grunze et al. 1996), that which is inappropriate. They therefore have a gain-control effect on ongoing processing, as shown in Figure 3c (based on data in Fox et al. 1990). (2) They let calcium into the cell, and thus play a major role in the cascade of processes that underlie learning. (3) They open less rapidly than non-NMDA-channels, and open for longer. Note, however, that direct application of NMDA to neural circuits can activate rhythmic bursting (Daw et al. 1993), and that NMDA currents may have a rapidly decaying com- ponent with a time constant short enough to support fast bursting and synchronization (Jensen et al. 1996). (4) They have a rich set of sites that regulate channel function. Figure 3 shows only a few of them. (5) They are widely distributed across all cortical regions, and are especially dense in the hippocampus, basal ganglia, and pre-frontal cortex (Monaghan et al. 1989). (6) At the cellular level they form synapses on both pyramidal cells and on inhibitory interneurons (Grunze et al. 1996), and in the case of pyramidal cells they are predominantly located on distal dendrites (Siegel et al. 1994), which supports the hypothesis that it is predominantly the distal dendrites of pyramidal cells that receive long-range contextual input (Körding \& König 2000). (7) They have several subtypes, with subtly different physiological properties (McBain \& Mayer 1994). NMDAreceptors on pyramidal projection cells and on local circuit interneurons could therefore be of different subtypes (Grunze et al. 1996; Monyer et al. 1994).

Clearly, our hypothesis concerning the role of NMDAreceptors in cognitive coordination requires further development and test. Additional support discussed in section 5.2 concerns their relevance to the high-frequency synchronous rhythms that have been related to both cognitive coordination and schizophrenia.

\subsection{Coordinating interactions have general relevance for cognition}

Studies of vision are prominent in the evidence cited above because they relate neurophysiology and psychophysics in great detail. Higher cognitive functions are likely to be even more dependent on context-sensitivity and dynamic grouping, however. An empirical argument for supposing that such coordinating interactions have general relevance for cognition is that the mechanisms by which they could be implemented (such as those shown in Figs. 2 and 3) are widely distributed throughout the cortex. Furthermore, as will be outlined in section 5, EEG (electroencephalogram) recordings provide evidence that high-frequency synchronization plays an important role in various cognitive functions.

\section{NMDA-antagonists impair cognitive coordination and are schizomimetic}

If cognitive coordination depends upon ion flow through NMDA-channels then it should be disrupted when they are blocked. The effects of subanaesthetic doses of a class of drugs that include PCP and ketamine support this prediction as they produce their psychotomimetic effects via competitive or non-competitive antagonism with NMDAchannels ( Javitt \& Zukin 1991). For brevity we refer to this disorder as PCP-psychosis. Many reviews of PCP-psychosis and the associated glutamatergic hypothesis of schizophrenia are available (Coyle 1996; Ellison 1995; Goff \& Coyle 2001; Javitt \& Zukin 1991; Jentsch \& Roth 1999; Olney \& Farber 1995; Olney et al. 1999; Tamminga 1998). Only particularly relevant findings are summarized here. The schizomimetic effects of NMDA-antagonists suggests that schizophrenia may involve under-activity of NMDA-receptors, so evidence on this will also be discussed. Though PCP-psychosis seems more similar to schizophrenia than any other drug-induced psychosis, they are not claimed to be identical. Abi-Saab et al. (1998) give reasons for expect- 
Phillips \& Silverstein: Convergence of perspectives on cognitive coordination in schizophrenia

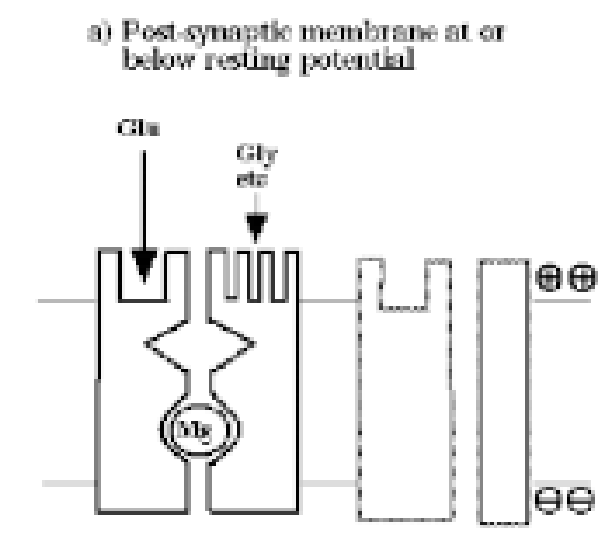

\section{b) Post-ssnaptic netembane abow resting potential}
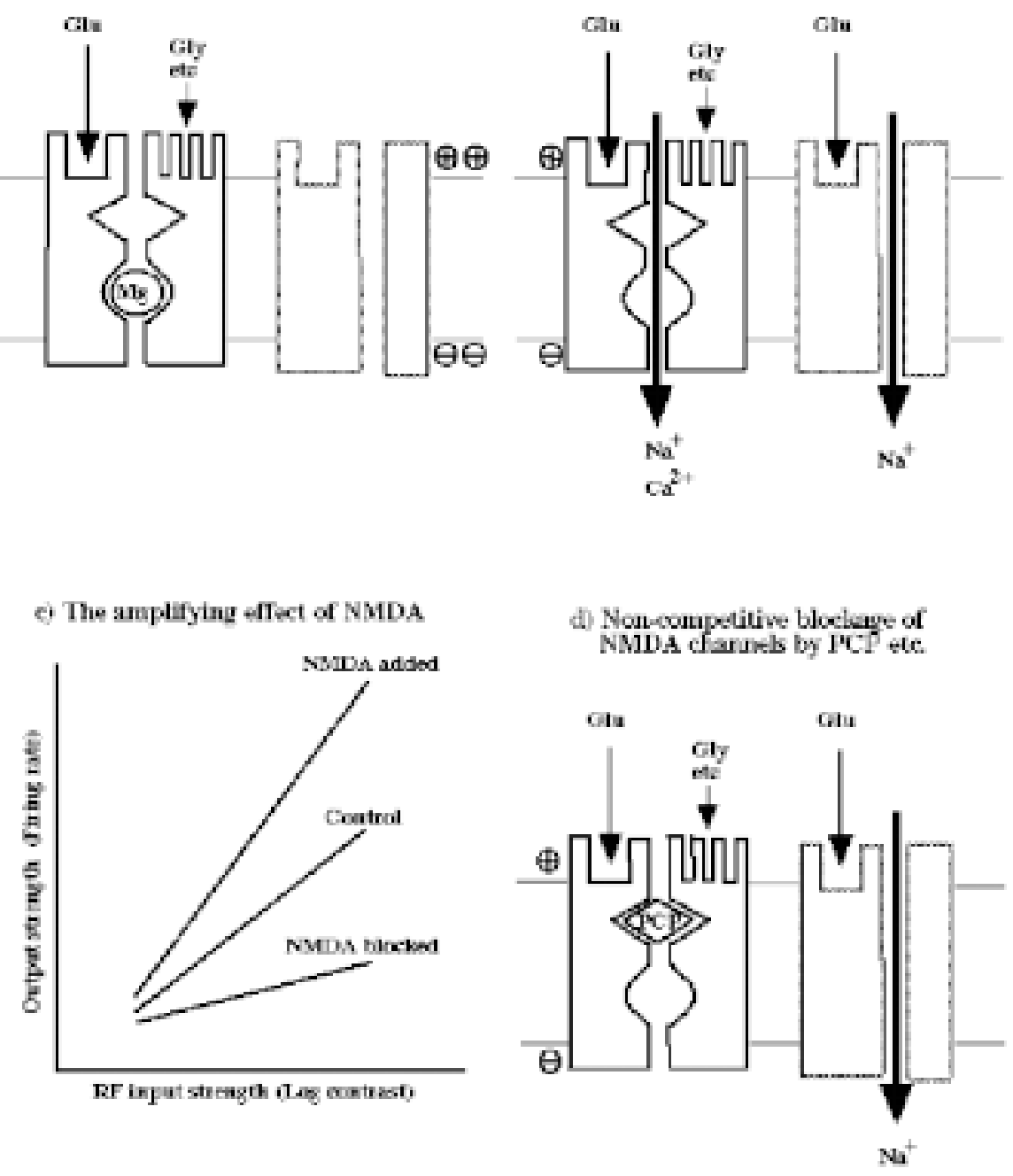

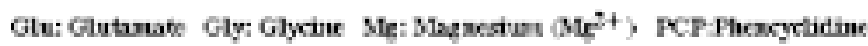

Figure 3. Outline of the voltage-dependency of NMDA receptor synaptic channels, emphasizing their amplifying function. NMDA receptor channels are shown in black outlines, non-NMDA (AMPA and KA) in grey outlines. Outward ion flow not shown. NMDA-channel function is regulated by several other sites in addition to those shown. (a) When the post-synaptic membrane is at or below resting potential, NMDA-channels are blocked by magnesium ions, which reduces ion flow through these channels when glutamate or NMDA binds to the receptor. (b) When the post-synaptic membrane is raised above resting potential, for example, by flow through non-NMDAchannels, the magnesium block is released, so ions then flow through these channels when glutamate binds to the receptor. (c) The amplifying effect of NMDA function, as shown by iontophoretic application of NMDA or of APV which blocks binding to the receptor. Graph based on the responses of cells in cat visual cortex to visual stimuli at their preferred orientation and of varying contrasts (Fox et al. 1990). (d) Binding to the PCP receptor site in NMDA-channels by phencyclidine, or its analogues ketamine, MK-801, and so on, reduces ion flow through those channels, but does not prevent flow through non-NMDA-channels.

ing differences, and conclude that the psychosis induced by NMDA-antagonists best models symptoms of cognitive disorganization including thought disorder. As we will show, it may also have relevance to other symptoms, however. Our focus here is therefore on the cognitive disorganization produced by reducing the activity of NMDA-receptor channels. NMDA-receptor antagonists, such as PCP, can influence other neurotransmitter systems also, however, so the complex psychopathology produced may also include effects due to those other influences.

Effects of NMDA-antagonists in humans are summarized in Table 1 . Acute effects of a single subanaesthetic dose occur within minutes and last a few hours; chronic effects of repeated doses can be long lasting. Their similarity to effects seen in schizophrenia is both striking and surprising, given the very different developmental courses of the two conditions. The effects of acute exposure to NMDA-antagonists show that most of the symptoms and deficits seen in schizophrenia can be produced without long-term structural damage. The relevance of NMDA-antagonists to schizophrenia is further strengthened by evidence that they provoke relapse in schizophrenic patients, who are highly sensitive to them (Allen \& Young 1978; Bakker \& Amini 1961; Lahti et al. 1995b; Malhotra et al. 1997; Tamminga 1998). PCP-psychosis also resembles schizophrenia in that it is age dependent, with its effects being much less appar- 
Phillips \& Silverstein: Convergence of perspectives on cognitive coordination in schizophrenia

Table 1. Effects of NMDA-antagonist exposure in humans

\begin{tabular}{|c|c|c|}
\hline Effect studied & Acute Exposure & Chronic Exposure \\
\hline \multicolumn{3}{|l|}{ 1. Positive Symptoms } \\
\hline Delusions & Paranoia, Reference $^{b}$ & Reference, Influence, Grandiosity, Religious ${ }^{\mathrm{d}}$ \\
\hline Hallucinations & Visual $^{\mathrm{b}}$ Auditory ${ }^{\mathrm{e}}$ & Auditory d \\
\hline \multicolumn{3}{|l|}{ 2. Negative Symptoms } \\
\hline Negativism/Withdrawal & $\begin{array}{l}\text { Emotional withdrawal, } \\
\text { Psychomotor retardation }{ }^{\mathrm{a}, \mathrm{b}, \mathrm{e}}\end{array}$ & \\
\hline Affective Flattening & $\mathrm{Yes}^{\mathrm{a}, \mathrm{e}}$ & \\
\hline \multicolumn{3}{|l|}{ 3. Disorganized Symptoms } \\
\hline \multicolumn{3}{|l|}{ Conceptual Disorg. and/or } \\
\hline Thought disorder & Yes $^{\mathrm{b}, \mathrm{c}, \mathrm{p}}$ & Yes $^{\mathrm{d}}$ \\
\hline \multicolumn{3}{|l|}{ 4. Cognitive Deficits } \\
\hline Working Memory & $\mathrm{Yes}^{\mathrm{i}, \mathrm{m}}$ & Yes $^{\mathrm{n}}$ \\
\hline Long-Term Memory & $\begin{array}{l}\text { Verbal }^{\mathrm{q}} \text { Semantic } \\
\text { Free Recall Declarative }{ }^{\mathrm{m}} \\
\text { Fecog. Memory }\end{array}$ & \\
\hline Executive Functioning & $\begin{array}{l}\text { WCST }^{\mathrm{b}, \mathrm{j}} \text { Verbal Fluency } \\
\text { Thinkinga,c Concrete } \\
\text { Matrices }^{\mathrm{a}}\end{array}$ & \\
\hline Attention & $\begin{array}{l}\text { Selective Attentiona Digit Span } \\
\text { Stroop Test }{ }^{\mathrm{a}} \text { Increased Reaction } \\
\text { Time }^{\mathrm{o}}\end{array}$ & $\begin{array}{l}\text { Reduced Attention Span }{ }^{1} \text { Trail marking } \\
\text { Test }(\mathrm{B})^{\mathrm{n}}\end{array}$ \\
\hline Eye Movements & Smooth Pursuitg & \\
\hline Context Processing & Auditory and Visual ${ }^{\mathrm{f}}$ & \\
\hline Perception & $\begin{array}{l}\text { Visual }^{\mathrm{a}} \text { Perceptual Awareness } \\
\text { Perceptual Constancy }\end{array}$ & \\
\hline 5. Affect & Anxiety ${ }^{\mathrm{b}}$ Euphoria \& Disorganization ${ }^{\mathrm{e}}$ & Anxiety, Euphoria, Labile \\
\hline 6. Brain Blood Flow & Increase in $\mathrm{PFC}^{\mathrm{e}}$ & Decrease in $\mathrm{PFC}^{\mathrm{k}}$ \\
\hline
\end{tabular}

${ }^{a}$ Bakker \& Amini (1961) brystal et al. (1994) cAdler et al. (1998a) dAllen \& Young (1978) eVollenweider et al. (1997b) ${ }^{\mathrm{f}}$ Umbricht et al. (2000) g Weiler et al. (2000) halhotra et al. (1996) ${ }^{\mathrm{i}}$ Ghonheim et al. (1985) jKrystal et al. (2000) ${ }^{\mathrm{k}}$ Hertzman et al. (1991) ${ }^{\mathrm{l}}$ American Psychiatric Association (1989) ${ }^{\mathrm{m}}$ Newcomer et al. (1998) ${ }^{\mathrm{n}}$ Cosgrove \& Newell (1991) ${ }^{\circ}$ Rosenbaum et al. (1959) pAdler et al. (1999)

qHarborne et al. (1996).

ent in immature subjects (Karp et al. 1980). PET-scans (positron emission tomography) show that in healthy volunteers these drugs produce an acute increase in metabolic activity in PFC, anterior cingulate, and in parietal and sensorimotor cortex, together with decreases in hippocampus and lingual gyrus, with effects 20-40 minutes later in the thalamus and cerebellum (Breier et al. 1997; Lahti et al. 1995a; Tamminga et al. 1995; Vollenweider et al. 1997a, 1997b). Repeated administration produces a chronic decrease in PFC activity, however, so chronic PCP-psychosis may provide the best model of schizophrenia (Jentsch \& Roth 1999). In vivo brain imaging, post-mortem neurochemistry, and clinical pharmacology all provide evidence of glutamatergic abnormalities in schizophrenia (Hirsch et al. 1997; Tamminga 1998). Furthermore, a recent study of post-mortem hippocampal tissue suggests that it may not be the number of NMDA-channels that is pathological, but their subunit composition (Gao et al. 2000). Further evidence for NMDA-hypofunction in schizophrenia are given in the reviews cited above. The NMDA-antagonist ketamine produces deficits very similar to those seen in schizophrenia in both the mismatch negativity paradigm, a reflection of pre-attentive contextual interactions, and in the AX version of the Continuous Performance Task, a measure of post-attentive contextual interactions (Javitt et al. 2000; Umbricht et al. 2000). The schizomimetic effects of
NMDA-antagonists, including reductions in context-sensitivity, are therefore well established. We therefore need to determine how these effects occur.

Some cognitive deficits in both PCP-psychosis and schizophrenia may be due to generalized overexcitation and any consequent neuropathophysiology, rather than being direct consequences of NMDA-hypofunction. The neurotoxic effects of chronic NMDA-hypofunction are well established (Ellison 1995; Olney \& Farber 1995; Olney et al. 1999). This may contribute to long-term psychopathology but is unlikely to explain the acute psychological effects of PCP, which occur within minutes and are transient. Furthermore, neuronal degeneration in schizophrenia often seems too minimal to produce the deficits observed (Tamminga 1998). The neuronal degeneration that does occur can be explained as resulting from overactivity due to the failure of NMDA-receptors to facilitate excitatory input to inhibitory interneurons (Coyle 1996; Ellison 1995; Olney \& Farber 1995). Interactions with the dopamine system could also lead to overactivity. Some cognitive deficits may thus be consequences of generalized overactivity (Coyle 1996; Olney et al. 1999). It is hard to see how this can explain all of the observed deficits, however. First, it is hard to see how generalized overactivity can be used to predict the specific changes in context-sensitivity and perceptual grouping emphasized throughout this article. Increases in activity, for 
example, as reflected by neuroimaging, are often assumed to be functional, not malfunctional. Second, impaired sensory gating does not reflect general overactivity because response latencies and amplitudes to individual stimuli are unaffected (Catts et al. 1995; Javitt et al. 1996). Third, neuroimaging does not show general overactivity in psychosis. Fourth, if the effects of NMDA-hypofunction were largely due to a consequent under-activity of inhibitory cells, then agonists for inhibitory cells might be prominent in pharmacotherapy, but they are not (Olney et al. 1999). Finally, many NMDA-channels are localized on pyramidal cells (Daw et al. 1993; Grunze et al. 1996; Siegel et al. 1994), and thus have facilitatory rather than inhibitory effects on pyramidal cell activity. Direct evidence that they facilitate sensory gating will be outlined below.

The role of NMDA-channels in learning is widely accepted, and learning is impaired in both PCP-psychosis and schizophrenia, so some of the other cognitive deficits may be a secondary consequence of impaired learning. This cannot account for many of the symptoms observed, however, as effects on immediate experience are prominent, which is to be expected, given the direct effect of NMDA-channels on glutamatergic activity. Furthermore, although there are some similarities between amnesia and schizophrenia, there are also clear differences (Heinrichs 1993). Impaired learning is thus more likely to be a consequence than a cause of impaired coordination.

Some of the cognitive effects of NMDA-dysfunction will arise as consequences of interactions between the NMDAsystem and other neurotransmitters, such as dopamine and serotonin, and so on (Olney et al. 1999). Glutamatedopamine interactions are complex (Coyle 1996; Jentsch \& Roth 1999). Various studies suggest that reduced NMDAreceptor function can facilitate sensitization of dopamine systems (Duncan et al. 1999). NMDA-antagonists produce dopamine dysregulation (Jentsch \& Roth 1999; Jentsch et al. 1997a; Moghaddam et al. 1997), for example, and these effects include an acute increase in the use of dopamine in dorsolateral PFC, followed by a chronic decrease (Jentsch et al. 1997a; 1997b; Moghaddam et al. 1997). This is compatible with theories proposing a chronic reduction in prefrontal dopamine leading to excessive mesolimbic dopamine in schizophrenia (Davis et al. 1991; Jentsch \& Roth 1999). Conversely, dopamine may be involved in regulating NMDA-activity (Greengard et al. 1998; Olney et al. 1999), and there is evidence that antipsychotic drugs acting through the D2 dopamine receptor can enhance NMDA function (Goff \& Coyle 2001).

Some cognitive deficits resulting from NMDA-hypofunction are therefore likely to be due to interactions with other neurotransmitter systems. Some are likely to be directly due to NMDA-hypofunction, however, as that directly alters glutamatergic transmission. The challenge before us is to sort out which is which. One way to do this is via computational theories that relate specific properties of NMDA-channels, such as their voltage-dependence, to specific cognitive functions (e.g., Kay et al. 1998; Phillips et al. 1995; Tononi et al. 1994; 1996). PCP-psychosis has from the start been interpreted in terms of processes that somehow "integrate" incoming information (Bakker \& Amini 1961; Luby et al. 1962). If cognitive coordination depends on NMDA-channel activity as proposed here, then this can explain why incoherent, irrelevant, and fragmented perceptions and thoughts arise from NMDA-hypofunction.
Another way to test hypotheses concerning direct effects of NMDA-dysfunction is to test specific predictions that they make concerning cognitive changes, and substantial progress has already been made in this direction (e.g., Javitt et al. 1996; 2000; Umbricht et al. 2000). Evidence on the direct effects of NMDA-hypofunction comes from studies of the effects of NMDA-antagonists on sensory gating in auditory cortex. Impaired sensory gating occurs in both schizophrenia and PCP-psychosis (Jentsch \& Roth 1999), and has been studied using mismatch negativity (Javitt et al. 1996). The response to an auditory stimulus that occurs as a deviant within a sequence of repetitive stimuli is greater than if it occurs as a repeated stimulus. This, and related effects of stimulus context, are much reduced in schizophrenia (Catts et al. 1995; Javitt et al. 1996; Judd et al. 1992), and it has been shown that NMDA-hypofunction can produce this effect (Javitt et al. 1996). NMDA-antagonists were infused into a microregion of primary auditory cortex in awake monkeys, and multichannel electrodes recorded neural activity in response to repetitive and deviant auditory stimuli. NMDA-antagonists removed the effect of stimulus context without affecting the primary obligatory response to the individual stimuli (Javitt et al. 1996). Neuronal degeneration, dopamine dysregulation, and impaired learning are all unlikely to be involved in this case, so this is evidence that reduced context-sensitivity in sensory cortex can be directly due to NMDA-hypofunction. Together with the studies of Umbricht et al. (2000), showing that ketamine impairs contextual interactions in healthy volunteers, these results therefore provide strong support for the perspective we advocate.

\section{Cognitive coordination is impaired in schizophrenia}

\subsection{Deficits in perceptual grouping}

In a study of the subjective experiences reported by patients, Cutting and Dunne (1989) found that perceptual dysfunctions were the most invariant feature of the early stage of schizophrenia. Goodarzi et al. (2000) found that, in people scoring highly on schizotypy, local processing dominates global processing, thus producing a deficit in perceptual Gestalt organization. Gestalt organization is a paradigmatic example of cognitive coordination. Many studies show that it is impaired in schizophrenia (Cox \& Leventhal 1978; Knight 1984; 1992; Knight \& Silverstein 1998; Place \& Gilmore 1980; Rabinowicz et al. 1996; Silverstein et al. 1996a; 1996b; 1998a; 2000). These impairments are a specific feature of the illness itself. They are not due to a generalized cognitive deficit because patients perform relatively better than normal in conditions where grouping would interfere with performance (Place \& Gilmore 1980; Silverstein et al. 1996a, as shown in Fig. 4). They are not an epiphenomenon of treatment, because: (1) there is no relation between either oral dose or blood level of depot medication and performance on perceptual organization tasks (Knight 1992); (2) patients medicated using traditional neuroleptics, and unmedicated patients, do not perform differently on these tasks (Rabinowicz et al. 1995); and (3) impairments have been demonstrated in unmedicated patients (Frith et al. 1983).

Perceptual grouping is far from being completely absent in schizophrenia, however. Patients perform adequately 
Phillips \& Silverstein: Convergence of perspectives on cognitive coordination in schizophrenia

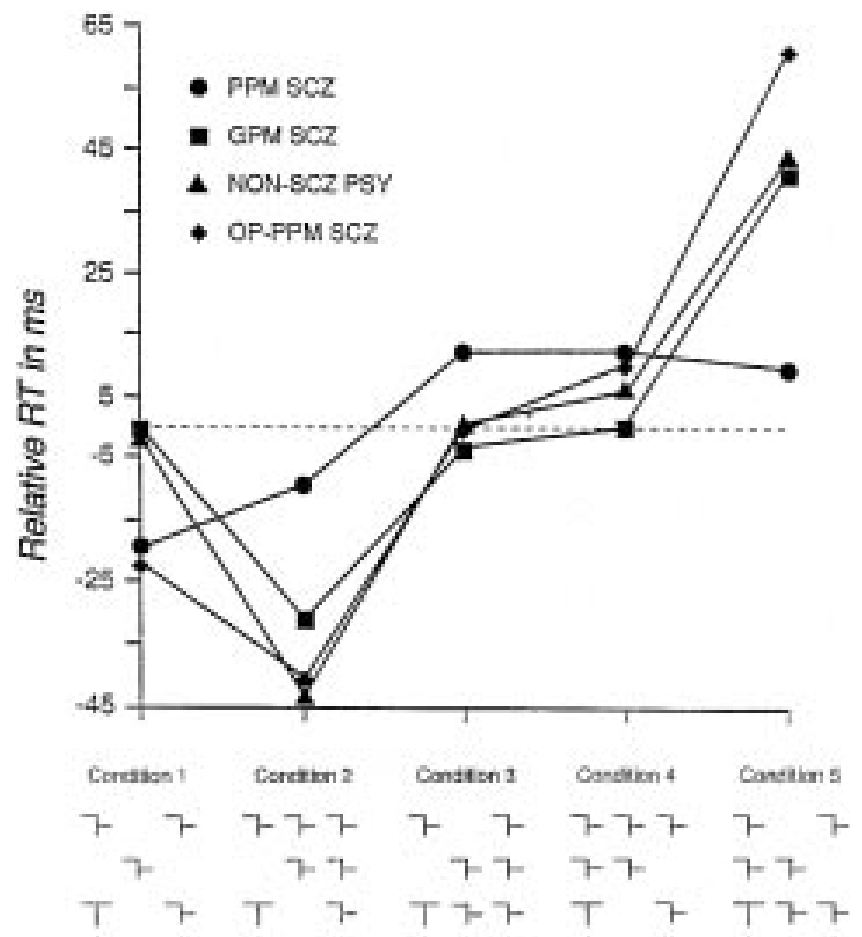

Figure 4. The effect of five different grouping conditions on mean reaction time to decide whether the letter in an array is a $\mathrm{T}$ or an $\mathrm{F}$ for four different groups of subjects. All, except for the inpatient poor premorbid schizophrenic group, show the same effects of grouping condition as that obtained with normal subjects. $\mathrm{PPM}=$ poor premorbid; $\mathrm{SCZ}=$ schizophrenia ; $\mathrm{GPM}=$ good premorbid; PSY = psychosis; OP = outpatient (data from Silverstein et al. 1996a).

when the task involves stimuli with continuous contour or strong configural properties (Chey \& Holzman 1997; Knight \& Silverstein 1998; Silverstein et al. 1998d). In the absence of strong cues to grouping, however, schizophrenia patients show clear impairments in combining noncontiguous elements (e.g., dot or line patterns) into perceptual wholes (Cox \& Leventhal 1978; Place \& Gilmore 1980; Silverstein et al. 1996a; 1998a; 2000). Patients are also impaired in their ability to alter perceptual organization of ambiguous stimuli based on current context (Silverstein et al. 1996b), or to develop perceptual organization for unstructured patterns after repeated exposure (Place \& Gilmore 1980; Silverstein et al. 1998a). Furthermore, the performance deficit shown by patients was removed by strengthening the contextual input, suggesting that they required stronger context than normal (Silverstein et al. 1996a). In addition, symmetrical stimuli comprised of noncontiguous components are not processed as groups by schizophrenia patients without strong top-down influences that use context (Knight 1992; Silverstein et al. 1998a). Schizophrenia thus involves a reduced ability to organize activity into coherent groups, but this only impairs performance when cues to grouping are weak in some way.

Perceptual grouping depends on the combined operation of two different but mutually supportive processes: grouping through convergence in pre-specified feature hierarchies; and grouping through dynamic Gestalt organization (Watt \& Phillips 2000), which involves processes that create novel groupings that can be specified only after the input is known. The computation of local motion signals can be pre-specified, but the perception of the global coherent motion of a whole surface requires dynamic grouping because it depends upon working out which local motion signals arise from a single surface in each particular instance (Watt \& Phillips 2000). Our perspective therefore predicts that global, but not local, motion perception will be impaired in schizophrenia. A recent psychophysical study supports this prediction (Chen et al. 2001), and fMRI data show that activity in higher motion perception areas of visual cortex is much reduced in neuroleptic-naive patients (Tost et al. 2001). These findings provide strong support for our perspective.

\subsection{Deficits in contextual disambiguation}

Many paradigms have been developed to study different forms of contextual disambiguation and, though diverse, all require detailed contextual information to modify the processing of information about other things. We first discuss effects that can plausibly be explained as depending on executive functions, then those that are more plausibly explained in terms of other functions. Reduced context-sensitivity in schizophrenia has been found in many paradigms designed to assess executive function and selective attention, including: the Wisconsin Card Sort Test (WCST) (Gold et al. 1997); the Stroop task (Cohen et al. 1999a; Perlstein et al. 1998); various versions of the Continuous Performance Task (CPT) (Cohen et al. 1999a; Pigache 1999); the anti-saccade task (Curtis et al. 2001); and N-back tasks (Perlstein et al. 2001). Such findings have often been replicated (e.g., Servan-Schreiber et al. 1996), and include deficits in first-episode as well as in chronic patients (Riley et al. 2000). Abnormalities in the Stroop paradigm are complex, but can be interpreted as arising from an impaired ability to focus attention on those aspects of the stimulus that are relevant to the task (Barch et al. 1999). This reduced sensitivity to task-context is most closely associated with disorganized symptoms (Barch et al 1999), that is, with the same sub-group of patients who show reduced sensitivity to stimulus-context. Contextual disambiguation is also impaired in language comprehension (Cohen et al. 1999a; Hoffman \& McGlashan 1993; Kuperberg et al. 1998), and production (Barch \& Berenbaum 1997; Patterson et al. 1986; Spitzer et al. 1994). These deficits have also been explained in terms of a component of WM that stores task relevant contextual information in the PFC (Cohen \& ServanSchreiber 1992).

Reduced context-sensitivity has also been found in many paradigms testing non-executive functions, including: reduced effects of irrelevant letters flanking a target letter in a choice reaction-time task ( Jones et al. 1991); reduced prepulse inhibition (Adler et al. 1998b); reduced use of context for phonemic disambiguation (Rochester 1978); reduced effects of context in weight perception and related paradigms (reviewed in Magaro 1980); and reduced mismatch negativity amplitudes, which is an event-related potential that reflects preattentive perceptual processing (Catts et al. 1995; Javitt et al. 1996; 2000). Delayed-tone matching is also impaired, and is due to impaired sensory comparison of a current tone with the immediately preceding context, rather than to increased distractibility (Rabinowicz et al. 2000). The decreased amplitude of mismatch negativity 
scalp potentials to unattended stimuli are found in the same patients that show deficits in the CPT (continuous performance task) ( Javitt et al. 2000). The contextual interactions involved in the mismatch negativity deficit are pre-attentive, whereas those involved in the CPT deficit are post-attentive. Both deficits of contextual processing could arise from the same kind of underlying pathology, however, and this view is supported by the finding, noted in section 3, that similar deficits in both mismatch negativity and the CPT are produced in normal volunteers by ketamine (Umbricht et al. 2000).

Paradigms in which context is misleading (e.g., Jones et al. 1991; Kuperberg et al. 1998) provide strong evidence for a specific reduction in context-sensitivity, because in those paradigms context interferes with the performance of patients less than it does with that of control subjects. Reduced interference cannot be due to a general performance deficit.

Contextual disambiguation is therefore impaired in tasks that assess executive functions and in tasks that assess nonexecutive functions. Furthermore, as will be discussed further in section 4.4, deficits of context-sensitivity correlate most strongly with disorganized symptoms (e.g., Barch et al. 1999; Cohen et al. 1999a).

\subsection{Memory deficits}

Memory deficits are relevant here because, as the Gestalt psychologists emphasized, dynamic organizational processes are also important in memory functions. Both episodic and semantic memory deficits occur in schizophrenia, with the latter being relatively more impaired than in the amnesic syndrome (Chen \& McKenna 1996; Duffy \& O'Carroll 1994). In addition, the influence of contextual constraints between items in the recall of verbal material is reduced in thought-disordered patients (Maher et al. 1980). Long-term memory deficits are to be expected from our perspective for at least four reasons. First, NMDAchannels, synchronization, and coherence have all been implicated in long-term memory formation (Singer 1995). Second, context is important for retrieval from long-term memory. Third, it has been hypothesized that contextual interactions are important for both the learning of associations between features and the discovery of those features that are associated (Kay et al. 1998). Fourth, source memory and memory for affective significance both require the formation of assemblies that relate items to the relevant context.

Though less clear than long-term memory deficits, WM deficits have also been associated with schizophrenia (e.g., Park \& Holzman 1992). Digit span is reduced from about seven to about six digits (Gruzelier et al. 1988), and shortterm visual memory capacity is also reduced (Knight et al. 1985). Though such small impairments could easily be discounted, they may have important consequences for cognition under demanding or stressful conditions. WM depends upon dynamic grouping because it requires memory for novel combinations of familiar elements. Experiments with normal subjects show that only about three or four dynamic groupings can be held simultaneously in visual WM (Luck \& Vogel 1997), and these groupings could be signalled through synchronization (Lisman \& Idiart 1995; Luck \& Vogel 1997; Raffone \& Wolters 2001). Simulations of recurrent networks composed of physiologically realistic ele- ments show rigorously how NMDA-currents can select and maintain any novel subset from a set of familiar elements (Lisman et al. 1998). Furthermore, an independent analysis of the dynamics of synaptic transmission, for example, in PFC circuits, also suggests a critical role for NMDAreceptors in WM (Wang 1999). Working memory, NMDAcurrents, and dynamic grouping may therefore be closely related, and, if so, this could help explain subtle WM deficits in schizophrenia (Lisman et al. 1998). Dopamine dysregulation in PFC has also been related to WM deficits in schizophrenia (e.g., Arnsten 1998; Braver et al. 1999), so it is possible that NMDA-hypofunction could produce WM deficits both directly and by contributing to dopamine dysregulation.

\subsection{Relationships between cognitive coordination and clinical symptoms}

At the level of signs and symptoms, fragmentation of mental functions is prima facie evidence of impaired cognitive coordination. At a phenomenological level, this could give rise to experiences in which the individual components of mental or motor activity take precedence over any coherent or integrated pattern of activity. Reviews of phenomenological reports by patients with schizophrenia support this view (Carr \& Wale 1986). Reports of fragmented experience can be found throughout the clinical literature, and they suggest that cognitive impairments often involve the reduced coordination of distributed activities.

Cognitive disorganization and thought disorder are more related than are other symptoms to each of the major aspects of cognitive coordination, for example, to sensorimotor gating (Perry et al. 1999), context-sensitivity in language perception (Kuperberg et al. 1998), context-sensitivity in language production (Spitzer et al. 1994), WM for task-relevant information (Cohen et al. 1999a), and knowing what goes with what within the semantic system (Goldberg et al. 1998). The relation of clinical status to perceptual grouping impairments has been studied in detail. They are more pronounced in patients who have been recently hospitalized for acute psychotic episodes compared to patients who have been living in the community for at least six months (Silverstein et al. 1996a). Furthermore, several studies show that patients with poor premorbid social function have more impaired perceptual grouping (Knight 1992; Knight \& Silverstein 1998; Silverstein et al. 1996a; 1998c). The Positive and Negative Syndrome Scale (Kay et al. 1987) has been used to relate impairments in cognitive coordination to specific symptom clusters. In one study, the best predictors of performance in a perceptual organization task were determined using a five factor solution (Lindenmayer et al. 1994). Only the cognitive or disorganization factor (consisting of conceptual disorganization, mannerisms and posturing, disorientation, difficulty in abstract thinking, and poor attention) was a significant predictor (Knight \& Silverstein 1998). Further evidence comes from a study in which symptoms of the Positive and Negative Syndrome Scale were related to the development of memory representations for configural and nonconfigural stimuli (Silverstein et al. 1998a). Once again, only the cognitive factor significantly predicted task performance. A reduced ability to develop a memory-assisted perceptual organization is therefore related to the disorganization component of symptomatology. A precise psychophysical study (Silver- 
Phillips \& Silverstein: Convergence of perspectives on cognitive coordination in schizophrenia

stein et al. 2000) also found that a reduced ability to perceive closed contours comprised of noncontiguous Gabor patches was related to higher levels of disorganized symptoms in chronic schizophrenia patients.

Evidence relating abnormal perceptual grouping to disorganized symptoms has also been obtained using the Thought Disorder Index (Solovay et al. 1986). Scores from 21 acutely psychotic schizophrenic patients showed that perceptual organization ability was correlated only with scores of disorganized and associative thought disturbance (Knight \& Silverstein 1998). These correlations were not due to a general performance deficit because degrees of disorganized and associative thought disturbance were not associated with baseline levels of perceptual task performance, and because the degree of perceptual organization impairment was not related to overall levels of thought disorder (summed across all four a priori factors). Further evidence of a relationship between abnormal perception and thought disorder or disorganized symptoms is that: (1) there is a significant correlation between an index of perceptual fragmentation on the Hooper Visual Organization Test (Lezak 1995) and degree of disorganized thinking (Osborn et al. 1994); and (2) thought disorder correlates with performance on the degraded stimulus version of the continuous performance test, which is a task that involves perceptual grouping (Knight \& Silverstein 1998; Nuechterlein et al. 1992; Silverstein et al. 1998b).

\section{Cortical rhythms, cognition, NMDA, and psychosis}

We argue above that cognitive coordination, NMDA-activity, and psychosis are related. Here we note that evidence relating each of these to high-frequency cortical rhythms strengthens the argument.

\subsection{High-frequency rhythms and cognition}

Gamma rhythms (30-80 Hz) are currently prominent in electrophysiological studies of cognition (Nunez 2000; Tallon-Baudry \& Bertrand 1999). They are relevant to theories of dynamic organization as they reflect synchronized activity that changes on a fast time-scale. They have been related to several aspects of dynamic organization, including Gestalt perception (e.g., Keil et al. 1999), attention (e.g., Tiitinen et al. 1993), and visual search and working memory (Tallon-Baudry \& Bertrand 1999). Gamma rhythms are first seen in infant scalp potentials at around eight months of age, which is when other evidence indicates that Gestalt perception shows major developments (Csibra et al. 2000). Though much of this research emphasizes gamma-band activity that is not phase-locked to stimulus events, activity that is phase-locked to stimulation is also relevant (Fries et al. 2001; Haenschel et al. 2000). Finally, coherent dynamics involves more than gamma-band activity (Habeck \& Srinivasan 2000). For example, beta rhythms (12-30 Hz) can tolerate longer conduction delays and still synchronize robustly, so they may be involved in coordinating activity between cortical regions (Bressler \& Kelso 2001; Bressler et al. 1993; Haenschel et al. 2000). Beta rhythms in the PFC could thus help coordinate activity in posterior cortex that is locally synchronized within the gamma-band (Kopell 2000).

\subsection{High-frequency rhythms and NMDA-receptor activity}

Mechanisms for the generation and control of high-frequency synchronized rhythms are complex, and not fully understood. As far as we can tell, inhibitory local circuit neurons play a major role in the primary generation of highfrequency rhythms, but NMDA-receptor activity has a role in controlling their strength, duration, and synchronization. Computational modelling clarifies these issues and suggests a role for NMDA-receptors (Jensen et al. 1996; Wright et al. 2001). Direct evidence relating NMDA-receptor activity to high frequency rhythms in auditory cortex (Javitt et al. 1996) was outlined in section 3 , but further evidence is also available. Whittington et al. (1997) showed that the transient high-frequency response of hippocampal pyramidal cells to brief input has a component mediated by NMDA and metabotropic glutamate receptors. Funahashi and Stewart (1998) report evidence that the duration of the gamma response of retrohippocampal cells to low-frequency repetitive input depends on NMDA-receptor activation. Faulkner et al. (1998; 1999) show that ketamine and other dissociative anaesthetics can disrupt the synchronization of gamma activity across separate sites and reduce the amount of beta-activity that is induced by gamma activity. Finally, Doheny et al. (2000) found that the NMDA-antagonist ketamine greatly reduced the difference between the gamma responses induced by novel and repeated stimuli in hippocampal slices. This is therefore analogous to the reduced effects of habituation and perceptual learning in schizophrenia.

Thus, although synchronized activity has often been thought to be due primarily to obligatory or driving connections, the above evidence suggests that non-linear contextual connections mediated by NMDA-receptors play an important role in controlling synchronization and thereby helping to coordinate cognitive activity as hypothesized by Phillips and Singer (1997a).

\subsection{High-frequency rhythms and schizophrenia}

As high-frequency synchronized rhythms are related to both dynamic organization and NMDA-receptor activity, our perspective predicts that they will be changed in schizophrenia. As a first approximation, we can hypothesize that gamma activity will be reduced, particularly in tasks requiring dynamic organization. Low-frequency rhythms may reflect more rigidly specified activity and may therefore be increased. A rapidly growing body of evidence supports these predictions. Using the degraded-stimulus CPT, Hoffman et al. (1996) found reduced gamma activity and increased low-frequency activity in neuroleptic-free schizophrenia patients. Clementz et al. (1997) found evidence relating sensory gating deficits to suppression of gamma activity. Kwon et al. (1999) studied the entrainment of the auditory EEG to clicks presented at 20,30, or $40 \mathrm{~Hz}$, and found that at $40 \mathrm{~Hz}$ this was much less in schizophrenia patients than in controls. Green et al. (1999) interpret the increased effects of visual masking that are associated with schizophrenia as being due to abnormalities in establishing high-frequency rhythms. Kissler et al. (2000) found that, when performing mental arithmetic, schizophrenia patients showed abnormalities in gamma activity, including general decreases of activity in the high gamma-range $60-71 \mathrm{~Hz}$. 
The report of exceptionally large $40 \mathrm{~Hz}$ rhythms during somatic hallucinations in a schizophrenia patient (Baldeweg et al. 1998) suggests that the hypothesis of reduced high-frequency activity is too simple. Abnormal patterns of high-frequency synchronization across cortical regions may also occur. Furthermore, different schizophrenic syndromes may show different EEG abnormalities. Gordon et al. (2001) related activity in narrow gamma-band (37-41 $\mathrm{HZ}$ ) responses to target and non-target stimuli in a standard auditory oddball paradigm. Overall, schizophrenic patients showed reduced gamma, but different syndromes were associated with different patterns of abnormality. Reality distortion was associated with increased gamma to targets (which may be an "over-attention" similar to that found by Baldeweg et al.). Psychomotor poverty was associated with reduced gamma to targets. Disorganization was associated with reduced gamma to non-targets, which Gordon et al. interpreted as reflecting a diffuse deficit involving contextsensitivity, with the other two syndromes reflecting distinct patterns of compensatory adaptation to this core deficit. For a more extensive review of evidence relating schizophrenia to changes in high-frequency rhythms see Lee et al. (2003b).

\section{Impairments of cognitive coordination are heterogeneous}

The depth of the heterogeneity in schizophrenia is unclear. It may be a family of disorders involving impairments of common underlying processes (Andreasen 1999). We will not know whether this is so until we identify any such process. Cognitive coordination is a candidate, and its disorders may be heterogeneous in several senses. They may be heterogeneous in that different aspects of coordination may be impaired to different extents and in different combinations. They may also be heterogeneous in the sense that disorders of coordination may be combined with disorders of various other aspects of mental function to produce differences both within and between classically defined psychiatric conditions.

Our hypothesis does not imply that schizophrenia is a homogeneous condition, but leaves much room for heterogeneity, both of neuropathology and of symptomatology. ${ }^{4}$ It suggests that some of the symptoms associated with schizophrenia are a direct consequence of NMDA-hypofunction, and that others are a consequence of associated pathophysiology, such as dopamine dysregulation or neuronal degeneration (Jentsch \& Roth 1999). We have focused on the attempt to characterize those that are directly due to disorders of coordination. Some non-psychotic visual disorders show that such disorders can be very specific. One example is strabismic amblyopia, which involves both impaired perceptual grouping (Polat et al. 1997) and impaired neuronal spike synchronization (Roelfsema et al. 1994). Impairments of particular aspects of cognitive coordination may also occur in some psychiatric disorders. For example, impairments to sensory gating can occur in mania (Franks et al. 1983). Impairments of many different aspects of cognitive coordination can also occur together, however, as in PCP-psychosis. The covariance of many aspects of cognitive coordination with thought disorder in schizophrenia also suggests that they are often jointly impaired.

The covariance of impairments in coordination with thought disorder does not imply that they are unrelated to other symptoms. First, NMDA-antagonists can rapidly induce hallucinations, delusions, and negative symptoms in normal volunteers, and profoundly exacerbate all of these symptoms in schizophrenic patients ( Jentsch \& Roth 1999). Second, in patients whose glycine levels are initially sub-optimal, glycine agonists (which then facilitate NMDA-channel opening) can improve negative symptoms (Goff et al. 1999; Heresco-Levy et al. 1999). Third, poor motor learning discriminates children at risk of developing schizophrenia from normal children (Mednick \& Silverton 1988), so disorders of motor coordination may be related to disorders of cognitive coordination. This is consistent with neurophysiological studies of motor cortex showing that spike synchronization occurs during normal sensorimotor coordination (Murthy \& Fetz 1996; Roelfsema et al. 1997), and with cognitive studies indicating that feature binding mechanisms are operative in action planning (Stoet \& Hommel 1999). Fourth, Carr and Wale (1986) present detailed arguments for viewing many hallucinations and delusions as compensatory adaptations to impairments of the processes that organize information into coherent wholes prior to the allocation of attention. Finally, internally generated mental activities also need to be linked to their source (Frith 1992), and if this linking fails, then various hallucinations and delusions may occur. ${ }^{5}$

Impairments of any particular aspect of cognitive coordination can also vary in severity. Consider perceptual organization. Patients with histories of poor premorbid social functioning show abnormalities that patients with good premorbid histories do not (Knight 1984; 1992; Knight \& Silverstein 1998; Silverstein et al. 1996a). This suggests that some perceptual abnormalities reflect more severe disability. This is supported by evidence that perceptual organization abilities on admission to a long-term psychiatric rehabilitation unit significantly discriminated between patients who could be discharged within three years from those needing longer hospitalizations (Silverstein et al. 1998e). Impairments of perceptual organization may therefore occur at different levels of severity (Silverstein \& Schenkel 1997). Subtle sensory gating abnormalities may also occur in the absence of psychosis because failure to attenuate the P50 psychophysiological response to the second of two auditory stimuli is distributed across affected and unaffected relatives of schizophrenic patients in a pattern consistent with autosomal dominant transmission (Adler et al. 1998b). More severe dysfunction of more aspects of perceptual processing may occur during states of acute psychosis. Evidence for this is that perceptual grouping disturbance is more prevalent among acutely psychotic poor premorbid inpatients than among outpatients with schizophrenia who have poor premorbid histories (Silverstein et al. 1996a), and that degree of perceptual organization disturbance in schizophrenia patients is significantly correlated with severity of disorganized symptomatology (Knight \& Silverstein 1998; Silverstein et al. 1998a; 2000). We therefore conclude that impairments of cognitive coordination can range from mild impairments of a few aspects to severe impairments of many.

The neurodevelopmental model of Hoffman and McGlashan (1993) may be useful in explaining why perceptual grouping impairments are found mainly among schizophrenia patients with poor premorbid functioning, and among such patients when they are exhibiting psychotic 
symptoms. Noting evidence of exaggerated developmental neuronal pruning, they proposed that corticocortical connectivity may be abnormal in schizophrenia in one of three ways, depending on the number of initial connections and the amount of later pruning that occurs. Low initial connectivity combined with normal pruning would lead to an early age of onset, whereas a normal amount of initial connectivity combined with abnormally extensive pruning would lead to good premorbid functioning and a fast onset of psychosis. This is consistent with the view that more global adaptive difficulties occur as a result of reduced density/connectivity earlier in life, and that more specific and serious manifestations of impaired connectivity and coordination occur later, when further reductions in connectivity create the potential for psychosis to emerge (Silverstein \& Schenkel 1997). These more serious manifestations of impaired connectivity and coordination could include both disorganized symptoms and the impairments in perceptual grouping with which they are associated (Knight \& Silverstein 1998; Silverstein et al. 1996a; 1998a; 2000).

Cognitive disorganization and disorders of holistic Gestalt processing, may also be involved in other conditions, such as autism and some other neurodevelopmental disorders. ${ }^{6}$ The tendency for autistic subjects to process inputs as sets of independent parts, rather than as coherent wholes, has been clearly shown in various cognitive domains, including visual perception, visuospatial construction, and language processing (Frith \& Happé 1994; Happé 1997; 1999). Furthermore, a mild variant of this cognitive style is seen in the parents of children with Asperger's Syndrome (AS), and is more characteristic of males than of females (Baron-Cohen \& Hammer 1997). A recent study found that of fourteen men diagnosed as autistic, seven met criteria for schizophrenia of the disorganized type (Konstantareas \& Hewitt 2001). Genetically specified variations in the strength of coordinating neuronal interactions may therefore play an important role in producing different cognitive phenotypes, and this is in agreement with KarmiloffSmith's (1998) argument that many neurodevelopmental disorders arise from impairments of cellular-level processes that are common to many brain regions, rather than to impairments that are localized in high-level cognitive modules with particular roles that are genetically prespecified.

\section{The hypothesis of impaired coordination is related to several other theories}

Jansen and Faull (1991) note that it is impossible to propose a model of schizophrenia without ignoring most of the data. It is also impossible to do so without ignoring most of the other theories! Though we have focussed on coordinating interactions within the cortex, we assume that dynamic organization and contextual disambiguation are also relevant to the function of other brain regions such as the basal ganglia, the limbic system, thalamus and cerebellum. All of these have been implicated in schizophrenia, and have been emphasized by many different theories of its pathophysiology. Though there is no space to discuss them here, our working assumption is that the coordination of activity, mediated in part via NMDA channels, is as important to their function as it is to that of the cortex. ${ }^{7}$

As our discussion of relevant theories is necessarily selective, we emphasize only those that have led us to the per- spective advocated. We discuss three groups of theories that have for the most part been developed separately, hoping to show ways in which they are mutually supportive.

\subsection{Theories emphasizing disconnection between cortical regions}

The disconnection hypothesis is built on conceptual foundations that combine ideas developed within the fields of theoretical neurobiology, cognitive neuropsychology, and neuroimaging (Dolan et al. 1999; Friston 1999). Schizophrenia is interpreted as arising from malfunctions of the dynamic interactions between cortical regions. It therefore contrasts with classical neuropsychological syndromes in that the disorder is not localized within specialized regions. The disconnection is not primarily anatomical but functional, that is, it is a disorder of the effective connectivity that mediates dynamic interactions. These change both on long and on short time-scales. The former relates to learning, and the latter to processing, and in particular to the dynamic modulation of effective connectivity. Symptoms of reality distortion and disorganization are seen as arising from a failure of the dynamic organizational processes that integrate activity across different regions. One example is the explanation of verbal hallucinations as a failure in selfmonitoring, as suggested by Frith (1992) and outlined in section 6. Another is that delusions of alien control can be seen as a failure to integrate the intention to act with the perceptual registration of the consequences of that action (Friston 1999). In relation to the neuropathophysiology it is suggested that changes in effective connectivity arise in part from over-activity of dopamine D1 receptors in the anterior cingulate gyrus (Dolan et al. 1999).

There are several similarities between this hypothesis and that presented here. (1) Both emphasize the distinction between functional segregation and functional integration (Tononi et al. 1994). (2) Both emphasize dynamic interactions between cortical regions. For example, the explanations for verbal hallucinations and alien control just outlined depend upon what we call dynamic grouping. (3) Both distinguish between linear and non-linear interactions, with the form of the non-linear interaction that they use being remarkably similar (compare Equation 2 of Friston et al. 1995 with Equation 2 of Phillips et al. 1995). In both approaches, this non-linear interaction is interpreted as modulating the response of cortical neurons to their inputs from other sources. (4) Both emphasize that long-term changes to synaptic connectivity result from changes to the dynamic modulation of short-term processing. Indeed, learning was the initial focus of the Coherent Infomax theory (Phillips et al. 1995), where analytically derived rules for synaptic plasticity were used for the contextual guidance of learning, and were shown to have detailed similarities to a form of synaptic plasticity for which there is physiological evidence (Phillips \& Singer 1997a). For an improved and even more physiologically plausible version of these learning rules, see Körding and König (2000).

There are also differences between the disconnection hypothesis as developed so far and the perspective presented here. (1) Friston et al. emphasize faulty modulation of long-term plasticity, whereas we primarily emphasize the immediate effects of modulatory interactions on the dynamic organization of processing, and interpret the longerterm changes in connectivity as being dependent on that. 
(2) They emphasize faulty interactions between cortical regions, whereas we also emphasize interactions within regions. (3) Though they distinguish between modulatory and obligatory connectivity (Friston et al. 1995), they do not emphasize the primary role of the modulatory connections in the dynamic coordination of activity, whereas we do. (4) As a consequence of the preceding, we put more emphasis on the NMDA-receptor and its possible pathologies than they do. Overall, the similarities are more fundamental than the differences, so we see the two approaches as complementary.

\subsection{Theories emphasizing impairments in cognitive coordination}

Our perspective extends the pioneering work of the Gestalt psychologists and Gestalt theory-influenced psychiatrists. For Matussek (1952/1987), the perceptual world of schizophrenia patients was characterized by a splitting of individual perceptual components from their natural context. This allowed normally incidental objects in the environment to take on unusual significance, and could relegate important stimuli to background status. Matussek reported cases of intense attentional focus on environmental details, and compared this to the heightened sense of an object that can occur under the influence of psychotomimetic agents such as mescaline. Experiences of attentional alteration, reduced awareness of surroundings, and an altered sense of reality and the self have been reported among nonpatients during states of "absorption" (Tellegen \& Atkinson 1974), and similar states occur in schizophrenia (Fisher 1976a; 1976b; Silverstein 1988).

A major difference between the transient states and psychotic episodes in schizophrenia is that the latter are not under the control of the individual, and thus have more serious long-term effects. These include giving atypical meanings to stimuli and the formation of new perceptual contexts that would further pull the patient away from reality. A classic example of this process is provided by one of Matussek's patients: "these objects seemed altered from the usual. They did not stand together in an overall context, and I saw them as meaningless details" (Matussek 1952/1987). Further research supports several of Matussek's hypotheses. For example, he hypothesized that the contextual weakening would vary depending on the severity of the illness. This has been confirmed by evidence reviewed earlier (Knight \& Silverstein 1988; Silverstein et al. 1996a; 1998a; 1998c; 1998e). Matussek also believed that awareness of appropriate contextual relationships could be brought about by drawing attention to relevant information, but that this awareness of context would be of only limited duration and would soon disintegrate. The ability to improve perceptual organization and other forms of context processing in schizophrenia through attentional manipulations has now been demonstrated experimentally (Silverstein et al. 1996a, Study 2 ), as has the temporary nature of the effect (Cromwell 1975; Kaplan 1974; Nuechterlein 1977).

A more recent view of schizophrenia based on similar principles is that of Carr and Wale (1986), who hypothesized that there are basic deficits in processes that organize both externally and internally generated activity. They view much of schizophrenic disorganization as reflecting the consequent failure to impose order on experience. They see a subtle impairment in cognitive organization as a stable as- pect of the illness, with more serious manifestations being due to sensory gating impairments becoming so severe that a system already deficient in organizing input is then overwhelmed by an even greater amount to be processed.

Many theories emphasize specific deficiencies in the use of context; for example, Gray et al. (1991) suggest that a key cognitive deficit in schizophrenia consists in the failure to relate specific associations to the context in which they occur, thus leading to impaired determinations of "relevance." Context plays a central role in the work of Cohen and colleagues (Braver et al. 1999; Cohen \& Servan-Schreiber 1992; Cohen et al. 1999a), who show how impairments in the CPT, the Stroop task, and lexical disambiguation could be due to malfunction of a WM system in the PFC. In an early version of their theory, these impairments were modelled as arising from reduced gain of units in a component of the model interpreted as being in the PFC (Cohen \& Servan-Schreiber 1992). In a later version, they are modelled as arising from increased noise levels in mesocortical dopamine (Braver et al. 1999). In both cases, the changes are hypothesized to arise from reduced dopaminergic modulation in the PFC. Our work builds in part upon theirs, but with important differences. Both approaches combine psychological, biological, and computational perspectives in their attempts to understand cognition in schizophrenia; but we emphasize general principles derived from the Coherent Infomax theory of cortical computation (Phillips \& Singer 1997a), and they emphasize connectionist simulations of performance in particular cognitive tasks. [For a detailed description of relations between computational theory and computational modelling, see Phillips and Singer (1997b, Table R2).]

Both approaches hypothesize that many cognitive deficits and clinical symptoms arise from changes to a few basic mechanisms involving context. In both, this is thought of as "information that is relevant to but does not form part of the content of the response" (Cohen \& Servan-Schreiber 1992 , p. 46), but whereas we see this as a basic aspect of cortical computation in general, they emphasize only task-relevant information stored in a WM component of PFC. The importance of the distinction between working memory and sensory levels of processing is not in question, having been clearly demonstrated long ago (Phillips 1974). The point we emphasize here is that contextual interactions are relevant to all levels. In support of this view we emphasize contextual interactions within perception. An important difference is therefore that Cohen et al. identify context with a particular set of representations, whereas we identify context with a widely distributed class of synaptic interactions. Another difference is that we see dynamic grouping as a fundamental form of coordination, and this relates our approach in very specific ways to high-frequency cortical rhythms as outlined in section 5. Finally, Cohen et al. emphasize dopaminergic modulation of explicit task-related information in PFC, whereas we emphasize NMDA-hypofunction. These are compatible. Indeed, they must be complementary, as the neurotransmitter systems involved are very different, but both have modulatory or coordinating functions. A major goal for future research is therefore to discover how they are combined.

\subsection{Theories emphasizing NMDA-hypofunction}

Several recent theories of pathophysiology in schizophrenia emphasize NMDA-hypofunction (Coyle 1996; Ellison 1995; 
Hoffman \& McGlashan 1993; Javitt \& Zukin 1991; Jentsch \& Roth 1999; Olney \& Farber 1995; Tamminga 1998), but they rarely relate that to cognitive coordination, and they do not emphasize the ability of NMDA-channels to provide gain-control through voltage-dependence. One research group that does both, however, is that of Javitt and colleagues. They interpret their studies of primary auditory cortex (Javitt et al. 1996) as showing that NMDA-channels are involved in modifying the strength of response to infrequent deviant stimuli within a sequence of repetitive stimuli. They note that this modification involves the contextual component of response rather than the obligatory stimulusinduced response, and interpret it as showing how a stimulus of one type can alter the response to a related but different stimulus. This component therefore provides a clear example of what we call contextual coordination. Javitt et al. (1996) also suggest further ways in which the role of inhibitory interneurons can be incorporated into an account of NMDA function and malfunction. Furthermore, although they demonstrate malfunction of preattentive processes in sensory cortex, they hypothesize, as we do, that analogous malfunctions can also apply to higher cognitive processes. Their studies using the CPT and mismatch negativity in both schizophrenia (Javitt et al. 2000) and in healthy volunteers (Umbricht et al. 2000) provide strong support for this hypothesis.

\section{Issues arising}

The perspective advocated here has implications for conceptions of cognitive neuropsychiatry, which when modelled too closely on cognitive neuropsychological studies of the effects of brain damage tends to neglect cellular physiology and neuropharmacology. This stands in stark contrast to the central role of pharmacotherapy in psychiatric practice. From our perspective, cognitive neuropsychiatry has much to gain by relating psychological disorders to neuroscience at the cellular level. Our perspective also encourages the application of new analytic techniques to neuroimaging data. Studies of cerebral blood flow have associated symptoms of disorganization with decreased perfusion in the right ventral prefrontal cortex and insula and with increased perfusion in the right anterior cingulate gyrus (Liddle et al. 1992). These are not the regions expected to be involved in the perceptual disorders outlined above. Failure to find changes in gross activity in perceptual regions is not incompatible with the hypothesis of impaired perceptual coordination, however, because both contextual disambiguation and dynamic grouping could occur with little or no change in gross activity. This emphasizes the need to consider not just gross activity within each region, but also its fine spatiotemporal structure both within and between regions. Techniques for imaging the extent to which brain regions interact (Tononi et al. 1998a) may therefore be relevant to psychosis, particularly when applied to data with high temporal resolution. Similarly, techniques for imaging synchronization on a fast time scale using EEG data (Phillips \& Pflieger 2000; Tallon-Baudry \& Bertrand 1999), and for distinguishing modulatory from obligatory intracortical interactions using fMRI data (Friston et al. 1995), may also have much to contribute.

The central hypothesis examined in this target article is that disorganization in some forms of psychotic cognition arises from reduced ion-flow through the NMDA family of glutamate receptors. Though both the cognitive and the neuropharmacological components of this argument are currently receiving much attention and support, neither is yet clearly established, and the wide-ranging implications of their mutually supportive relationship have been little discussed. We are well aware that our argument goes beyond what is currently widely agreed, and four central issues for commentary arise. (1) Does reduced ion-flow through NMDA-channels play a crucial role in the pathophysiology of cognitive disorganization in schizophrenia, and, if so, how does that relate to dopamine dysregulation and other pathophysiologies more widely thought to be involved in the disorder? (2) Is a reduction in context-sensitivity central to many of the cognitive impairments known to be associated with schizophrenia? Some investigators have suggested that context-sensitivity is not reduced in schizophrenia, but increased (David 1994). Should further tests show this to be so, then that will disprove what we advocate. (3) Does reduced flow through NMDA-channels play a central role in producing disorders of context-sensitivity? If it is shown that both occur, but apply to different patients, then that will be strong evidence against the position we advocate. (4) Does cognitive disorganization in PCP-psychosis provide evidence that normal cognition depends upon coordinating interactions that are implemented via NMDA-receptors?

Several closely related issues arise, the most important being as follows. (1) Can impairments of a fundamental underlying process in schizophrenia account for disorganization across cognitive, behavioral, and symptomatic domains, or are deficits in these domains best seen as being independent and capable of occurring in many different combinations? (2) Insofar as contextual interactions are involved in these disorders, is the relevant context only that which is provided by preceding information held in a working memory that is located in prefrontal cortex, or is it much wider than that, including current stimulus context and part-whole relationships? (3) Clinically, semantic associations are seen to be much less focussed in thought disordered patients, and this may be reflected by greater indirect semantic priming (Spitzer et al. 1993). How can our perspective be related to this? The first thing to note is that semantic associations require compositionality, which can be implemented via the dynamic grouping processes we have emphasized. These depend on contextual interactions, and mediated or indirect contextual interactions require primary input to the mediating items (Kay et al. 1998). Thus, if primary sources of activation, for example, from memory retrieval processes, noise, or other sources of activation is less focused in thought disorder, then more indirect associations can be predicted. (4) NMDA-receptors are widely thought to play a major role in learning, but not in ongoing processing, so how well supported is our claim that this view is mistaken and that NMDA-receptors also play a crucial role in ongoing processing? (5) The voltagedependence of NMDA-channels is usually ignored in discussions of their role in schizophrenia and PCP-psychosis, so how justified is our emphasis upon this property? (6) Are any other receptors plausible candidates for the role of implementing coordinating interactions between the pyramidal cells whose activity embodies our cognitive contents? (7) Which clinical symptoms are likely to be due to reduced context-sensitivity, and which are not? (8) How similar are 
the cognitive deficits that occur in PCP-psychosis and schizophrenia? (9) Is there a spectrum of cognitive phenotypes characterized by genetic variations in the balance between locally independent processes and context-sensitive holistic Gestalt interactions? (10) Is there evidence for underactivity of NMDA receptor channels in any other disorders involving cognitive disorganization? (11) Is the molecular and regional diversity of NMDA receptor channels (Kutsuwada et al. 1992) crucial, and is NMDA dysfunction limited to the cortex or does it also occur in other brain regions? (12) What pathophysiologies other than NMDA-hypofunction can impair the specific class of neuronal interactions that underlie cognitive coordination? Though we have focussed on NMDA-hypofunction, they could also be disrupted in other ways, such as via abnormal pruning (Hoffman \& McGlashan 1993; McGlashan \& Hoffman 2000), or via impaired consolidation of coordinating connections due to abnormal input during development, for example. (13) Do high-frequency rhythms provide a useful window on processes of dynamic organization and their impairment in schizophrenia? (14) Do postmortem and other studies indicate that cognitive disorganization is associated with reduced lateral and descending long-range connections of the kind that mediate contextual coordination via NMDA-receptors? (15) Why do some antipsychotic medications reduce cognitive disorganization? One recent review (Mechri et al. 2001) concludes that clozapine significantly reduces the ketamine-induced positive symptoms in schizophrenic patients. Another concludes that the effects of some atypical antipsychotics such as clozapine and olanzapine on NMDA receptors differentiates them from typical antipsychotics such as haloperidol (Goff \& Coyle 2001). It is therefore possible that some widely used antipsychotics enhance NMDA-receptor activity and thus reduce cognitive disorganization. If so, then agents more specifically designed to enhance NMDA activity, perhaps via the glycine modulatory site, may be even more beneficial. (16) Finally, how widely supported is our view that neuroscience at cellular and molecular levels should play a far greater role in cognitive neuropsychiatry than it has done so far in cognitive neuropsychology?

\section{ACKNOWLEDGMENTS}

The work of William A. Phillips on this paper was carried out in part during the tenure of a Visiting Fellowship at the Neurosciences Institute, San Diego. The research of Steven M. Silverstein was supported by grants from the Scottish Rite Schizophrenia Research Program and the Committee to Aid Research to End Schizophrenia. We thank Alan Baddeley, Steve Bressler, Peter Cahusac, Vaughan Carr, Eric Chen, Nigel Daw, Chris Frith, Jeffrey Gray, Peter Hancock, Dan Javitt, David Linden, John Lisman, Ronan O'Carroll, Mark Pflieger, Kenji Sakamura, Wolf Singer, Olaf Sporns, Jim Waltz, and Jim Wright, for their help and comments on the issues discussed. Special thanks to Peter Uhlhaas for help throughout this work, including the preparation of Table 1. Karl Friston, Michael Green, John Gruzelier, David Jentsch, and four other referees provided valuable advice.

\section{NOTES}

1. Consider illusory contours. Prima facie, this seems to be a case where context provides primary drive as it produces signals for things that are not there. This is clearly different to the examples shown in Figure 1a, right-most column, where context does not produce the perception of absent things. One way to handle such exceptions is to say that, to the extent that surrounding items produce primary drive, then they are, by definition, not acting as context. This view could be tested for coherence with the rest of our perspective in various ways, including investigations of the RF inputs to cells in visual cortex to see whether they included input from surrounds that produce illusory contours. Note that this is not merely a matter of finding out whether surrounds influence the activity of cells in visual cortex (we know that they do), it is a matter of finding out whether that influence is driving or modulatory. It could be both. Another way to view such exceptions is therefore to see them as the faint ghosts of modulatory inputs that in these exceptional cases produce output, possibly by amplifying selected components in background or noise activity. This latter view implies that the function of processes reflected in illusory contours is to amplify relevant but weak evidence that is actually present, rather than to signal the presence of absent things.

2. The creation, maintenance, and use of strategies that meet longer-term goals of the organism as a whole are computationally feasible because they are performed by systems that do not need to coordinate all the local details. Psychology and neurobiology have for many years provided evidence of such strategic or executive functions (Baddeley 1996; Fuster 1997; Miller \& Cohen 2001; Shallice 1988). Functional neuroimaging now adds to that evidence (Posner \& Raichle 1994), and shows that new theories are required to improve our conceptions of executive functions (Grafman et al. 1995). They involve several distinct sub-divisions of the PFC and anterior cingulate gyrus which operate via interactions with limbic, striatal, and posterior cortical regions. Though no definitive taxonomy is yet agreed, several classes of executive function are often postulated: modulation of schemas or routines in lower-level systems, including the inhibition of dominant response tendencies when they are inappropriate in the current context (Frith 1992; Liddle \& Morris 1991; Shallice 1988); modulation of perception and the control of attention in visuospatial and other modalities (Posner \& Raichle 1994); the maintenance or use of information in components of working memory (Goldman-Rakic et al. 2000); the formation and use of contextual associations in episodic and source memory (Janowsky et al. 1989: Shallice et al. 1994) and the assignment of affective significance (Damasio 1994; Petrides 1994; Robbins 1996); and the creative manipulation of information to solve novel problems (Owen 2000; Robbins 1996; Shallice \& Burgess 1996). Anatomical localization within PFC depends on semantic content, for example, with some parts being specialized for phonological information and others for visuo-spatial information (Goldman-Rakic et al. 2000). Different subdivisions of PFC may also be specialized for some executive functions rather than others. For example, some may be specialized for selectively disinhibiting lower-level routines, and others for the creative manipulation of information (Owen 2000).

3. Another aspect of executive function may be the gating of input to a component of WM in PFC that holds contextual information (Braver et al. 1999). It was clear from the original studies of visual short-term memory that interference due to subsequent activities was a major cause of short-term forgetting, and that central executive functions are important in controlling that interference (Phillips \& Christie 1977). The theory of Braver et al. (1999) offers a connectionist model that relates such interference to the gating of access to $\mathrm{WM}$ in $\mathrm{PFC}$.

4. The rich anatomical complexity of the glutamatergic system greatly increases the scope for heterogeneity. NMDA-channels are built from varying combinations of five different sub-units with varying regional distributions, developmental histories, and physiological properties (Watanabe et al. 1992). Different disorders, including those with genetic origins, could therefore effect these different subtypes of NMDA receptor in different ways; for example, schizophrenia is associated predominantly with abnormalities of the obligate NMDA-receptor subunit (MeadorWoodruff \& Healy 2000). The close interactions between the NMDA and dopamine systems implies that a primary pathology in either could lead to dysregulation of the other. Another possible source of heterogeneity is that the amount of neurodegeneration may depend upon whether glutamatergic overactivity due to 
reduced NMDA-channel activity on inhibitory cells outweighs underactivity due to reduced NMDA-channel activity on glutamatergic cells. This is compatible with evidence for cases of schizophrenia both with and without progressive degeneration (Benes \& Coyle 1998).

5. When patients experience verbal hallucinations they do not know that the words they experience are due to activity in those other parts of their cognitive system that generate things to be said (Frith 1992). Such hallucinations are possible because the sub-systems that instantiate the phonological and semantic forms of words can be activated by input from any of several different sources, some internal, some external. To signal the origin of activity within them on any particular occasion, that activity must be dynamically linked to the activity from which it arose on that occasion. Failure of this dynamic linking could produce the strange experience of having words in mind, but not as part of a larger pattern of activity that links them to their origin in other brain regions on that occasion. Verbal activity might then be experienced as coming from outside the self, and delusional beliefs might then reflect attempts to account for this experience.

6. Silverstein and Palumbo (1995) describe similarities between schizophrenia and nonverbal perceptual-organization-output disability, which is a severe form of nonverbal learning disability thought to fall within the autism spectrum (Rourke 1982). They suggest that this disorder also involves impaired stimulus organization mechanisms, and that studies of such disorders could compliment high-risk studies in the attempt to uncover the aetiology of schizophrenia. Furthermore, some learning disabled individuals have deficits in backward masking (Blackwell et al. 1983) and span of apprehension tasks (Tarnowski et al. 1986), which are often thought to be vulnerability markers for schizophrenia. Thus, there is evidence of common cognitive impairments in schizophrenia and some other neurodevelopmental disorders. It is not the case that all of these populations perform similarly simply by virtue of a general intellectual impairment. Certain developmental disorders exhibit a very different pattern of cognitive deficits. For example, patients with Williams Syndrome show increased rather than decreased global processing (Pani et al. 1999), when tested on the same perceptual organization task as used with schizophrenic patients by Silverstein et al. (1996a). Much may therefore be gained by comparing these disorders using relevant process-oriented designs (Knight 1992; Knight \& Silverstein 1998).

7. Discussing the role of the thalamus in the pathophysiology of schizophrenia, Patterson (1987) concludes "If one were to single out a brain structure that displayed the possibility for central 'timing' functions in brain, it would most likely be the thalamus." The basal ganglia are also often implicated in schizophrenia (Robbins 1990). Graybiel (1997) argues that just as they contribute to the coordination of motor output so they may also contribute to the coordination of cognitive activity. She argues that in both cases this coordination involves dynamic binding through the synchronization of firing patterns so as to produce appropriate and precisely timed sequences of activity. In their review of the functional architecture of the basal ganglia, Alexander and Crutcher (1990) conclude that "the functional integration that is widely assumed to occur within these circuits may prove to be based less upon the spatial convergence of functionally disparate pathways than upon the temporal coincidence of processing within pathways whose functional segregation is rather strictly maintained" (p. 270). All these views are highly consonant with that proposed here. One function of the limbic system is thought to include putting things in context while maintaining their individual identities, so that too, may involve the formation of contextual associations that are implemented in part by NMDA-receptors, which are particularly dense in the hippocampus.

\section{Open Peer Commentary}

Commentary submitted by the qualified professional readership of this journal will be considered for publication in a later issue as Continuing Commentary on this article. Integrative overviews and syntheses are especially encouraged.

\section{The ketamine model for schizophrenia}

\section{Murray Alpert and Burt Angrist \\ Department of Psychiatry, NYU School of Medicine, New York, NY 10016. murray.alpert@nyu.edu}

Abstract: This commentary compares clinical aspects of ketamine with the amphetamine model of schizophrenia. Hallucinations and loss of insight, associated with amphetamine, seem more schizophrenia-like. Flat affect encountered with ketamine is closer to the clinical presentation in schizophrenia. We argue that flat affect is not a sign of schizophrenia, but rather, a risk factor for chronic schizophrenia.

The Phillips \& Silverstein (P\&S) target article provides striking evidence for the explanatory power of drug models of psychotic psychopathology, although the paper is broader than the ketamine story, touching on clinical, cognitive, electrophysiologic, neuroanatomic, and other domains. We will focus on the clinical aspects of drug models of psychosis and compare ketamine with amphetamine, with some consideration of hallucinatory processes and loss of insight. In addition, we will touch on questions related to the role of flat affect. The target article should facilitate empirical study of important questions such as differences between ketamine and competing drug models.

At present, a number of drugs tied to different neurotransmitters have been shown to provoke psychotic symptoms. Early on, LSD produced considerable interest because of the tiny dose required for an induction. It was possible to imagine that a metabolic error could produce an endogenous intoxicant. Mescaline attracted interest because of its structural similarity to dopamine, and the authority of the transmethylation hypothesis. Neither LSD nor mescaline produced a clinical presentation that had the "look and feel" of schizophrenia. It appeared that different transmitters might provide some specificity for the different psychoses: Prolonged exposure to steroids could produce states that mimicked manic psychoses, and a ditran induction shared characteristics with the alcohol-withdrawal psychoses (Alpert et al. 1970).

The amphetamine model psychosis provides the "look and feel" of paranoid schizophrenia and nests nicely with the dopamine hypothesis of schizophrenia. Because of the risk of cardio-toxic effects, the rate of dosing of amphetamine must be slow, and the rate and duration of dose increase may be important for the amphetamine model (Alpert \& Friedhoff 1980). Many of these issues appear accessible to empirical study within the conceptual framework of the $\mathrm{P} \& S$ article. The amphetamine model seems more attractive than ketamine for a number of reasons. A model should demonstrate a schizophrenic presentation without altering consciousness, and ketamine is an anesthetic. It has a narrower range of action below a threshold for clouding.

In addition, the hallucinatory phenomena with ketamine are less compelling: The hallucinations are more mixed with illusions and there is a shift to visual compared with auditory changes. In surveys of hallucinations in schizophrenic patients (Alpert \& Silvers 1970 ), about $50 \%$ of the patients reported auditory hallucinations and about $20 \%$ reported visual hallucinations, and all of the patients with visual hallucinations also had auditory ones. Schizophrenic hallucinations are primarily verbal, of high intelligibility, and give the impression of "thoughts becoming audible." The alcoholic auditory hallucinations resemble "sounds becoming 
verbal." The hallucinating schizophrenic differs from the nonhallucinator in regard to cognitive style and semantic processing (Alpert et al. 1976). Amphetamine-associated hallucinations are phenomenologically like those in schizophrenia.

In their Figure 2, P\&S suggest horizontal and vertical neuroanatomic geometric models of neurotransmitter interactions for psychopathologic disturbances. It has been shown that sensory transduction of auditory sharpening mechanisms (lateral inhibition) may be affected by alcohol exposure in alcohol-withdrawal psychoses (Alpert \& Bogorad 1975). Similar processes may occur in schizophrenia, and could be accessible to psychophysical examination. In addition, hallucinators differ from nonhallucinating schizophrenics in regard horizontal organization of cognitive processing (Alpert \& Martz 1977). The P\&S model provides a reasonable context for investigation of these issues.

The loss of insight and other behavioral effects with amphetamine can be very impressive. Among Angrist's amphetamine subjects, one was reluctant to report his auditory hallucinations for fear that he would be locked away in a psychiatric hospital. He had predicted at baseline that he would experience verbal hallucinations as part of the amphetamine experience. When they occurred, he thought that he was becoming schizophrenic. Another subject spoke of "setups and traps" and rejected our attempts to reassure him. He was convinced that a gang was coming to the ward to get him. A third subject felt that he had received special enlightenment and had become a "prophet." He preached to the ward for about an hour (Angrist 1972; Angrist \& Gershon 1970). Loss of insight appears to be a direct, primary effect of the amphetamine induction, not the subjects' reaction to their perplexing subjective experiences. These important aspects of the induction do not appear to be duplicated in the ketamine model.

A ketamine induction, perhaps more than amphetamine, is associated with affective flattening. Although the DSM IV (Diagnostic and Statistical Manual IV, of the American Psychiatric Association) has added flat affect as a diagnostic criterion for schizophrenia, this may be an error. Flat affect appears early in life, perhaps years before schizophrenia appears (Knight \& Roff 1985), and may diminish at the time of an acute schizophrenic episode. Similarly, flat affect is reduced in cocaine abuse while hallucinations and delusions are markedly increased (Serper et al. 1995; 1996). Emotions appear to be intact in schizophrenics with flat affect (Alpert et al. 2000), and flat affect can be conceptualized as a disturbance in motor expression. Flat affect may worsen in treatment with typical neuroleptics but respond to treatment with atypical antipsychotic drugs, even while other psychotic signs remain. For these reasons, flat affect does not appear to be coherent with diagnostic signs for a schizophrenic episode. It may be conceptualized as a risk factor for schizophrenia rather than a sign of schizophrenia. Further, flat affect may represent a condition involving lowered dopamine turnover. The role of flat affect in ketamine model psychosis may represent complex interactions with dopamine. The P\&S article will help to clarify the actions of neurotransmitters in psychosis.

\section{Where the rubber meets the road: The importance of implementation}

\section{Deanna M. Barch and Todd S. Braver \\ Department of Psychology, Washington University, St. Louis, MO 63130. dbarch@artsci.wustl.edu http://iac.wustl.edu/ ccpweb/ tbraver@artsci.wustl.edu}

Abstract: Phillips \& Silverstein argue that a range of cognitive disturbances in schizophrenia result from a deficit in cognitive coordination attributable to NMDA receptor dysfunction. We suggest that the viability of this hypothesis would be further supported by explicit implementation in a computational framework that can produce quantitative estimates of the behavior of both healthy individuals and individuals with schizophrenia.
Phillips \& Silverstein (P\&S) put forth an interesting and provocative hypothesis as to the ways in which NMDA receptor dysfunction might lead to disturbances in cognitive coordination in schizophrenia. They do an elegant job of synthesizing psychological, computational, and neurobiological perspectives on the cognitive coordination construct and its underlying mechanisms. We are grateful that P\&S acknowledge our own work (with Jonathan Cohen and colleagues) as trying to achieve similar goals with regard to understanding cognition in schizophrenia (Braver et al. 1999). P\&S contrast their hypotheses to our theory, which suggests that one of the core cognitive deficits in schizophrenia is a dysfunction in the ability to represent and maintain context information, as a result of a disturbance in dopamine function in prefrontal cortex. P\&S highlight a potentially more fundamental mechanism of context processing (cognitive coordination in their model) that involves the NMDA-receptor and computational processing within, as well as between, cortical modules. As such, $P \& S$ suggest that deficits in the kinds of cognitive control mechanisms that are central to our theory could arise from disturbances in basic mechanisms that may be involved in processing throughout the entire brain. This contrasts with our theory, which focuses on processing mechanisms that more selectively involve dopamine interactions with prefrontal cortex, and on the cognitive capabilities that depend on such interactions. We have argued that disturbances in such mechanisms among individuals with schizophrenia give rise to relatively selective cognitive deficits that are most severe under particular task conditions.

We are excited by the prospect of a theory of cognition in schizophrenia that attempts the same integration of psychological, computational, and neurobiological perspectives that we have tried to incorporate in our work. An especially exciting prospect is the suggestion by $P \& S$ that their mechanism could account for deficits among individuals with schizophrenia, both on high-level cognitive tasks and in more basic sensory and perceptual domains. If this were true, it would constitute an advance upon our own theory, which is admittedly more constrained in terms of the phenomena for which it attempts to account. Phillips and colleagues have conducted computational studies demonstrating that NMDAreceptors have properties (i.e., their voltage-dependence) that allow these receptors to help organize processing and learning. However, a more convincing demonstration of the explanatory power of the P\&S model would be to explicitly demonstrate that a disturbance in the same mechanism could lead to changes in both high-level cognitive processing and sensory/perceptual (e.g., Gestalt grouping phenomena).

$\mathrm{P} \& \mathrm{~S}$ refer to a distinction between computational theory and computational modeling. Their theory seems to be rooted in the former approach. In contrast, our work has focused on the latter approach, using simulations of specific cognitive tasks. We would advocate that explicit simulations of cognitive tasks provide an useful means by which to compare and contrast theories such as ours and that of $\mathrm{P} \& \mathrm{~S}$. In particular, simulations of actual cognitive tasks enable quantitative estimates of the success with which a model can account for the relevant behavioral phenomena. Such estimates provide an objective metric by which to evaluate competing models. For example, one would judge the $\mathrm{P} \& \mathrm{~S}$ model to be a more successful model of cognition in schizophrenia than our own if, in addition to accounting for sensory/perceptual phenomena, the P\&S model could also account for the behavior of individuals with schizophrenia on tasks such as our AX version of the Continuous Performance Task (a task that our theory suggests is highly dependent on integrity of context processing functions) with the same degree of success that our model can.

Such explicit implementation may also help to identify task conditions that would help arbitrate between competing theories. For example, our simulation work has suggested that deficits in context processing among individuals with schizophrenia should be amplified under conditions in which context needs to be actively maintained in working memory and/or used to inhibit dominant response tendencies that are not appropriate for the task at hand. 
A number of empirical studies provide support for these model predictions (e.g., Barch et al. 2003; Cohen et al. 1999b; Javitt et al. 2000; Servan-Schreiber et al. 1996; Stratta et al. 1998). However, it is not clear from the level of description provided by $\mathrm{P} \& \mathrm{~S}$ whether their theory would also predict that such factors should influence the severity of cognitive deficits in schizophrenia. It is also possible that simulations of specific cognitive tasks in the $P \& S$ framework would identify other conditions that are especially dependent on their proposed NMDA-receptor mechanism. In our experience we have found that the process of simulating empirical phenomena forced us to refine and elaborate our initial conceptual hypotheses in ways that we could not have predicted ahead of time.

In summary, we are intrigued by the theory put forth by $\mathrm{P} \& \mathrm{~S}$ and encourage the authors to take this theory to the next level by providing an explicit computational implementation that can be compared with competing theories.

\section{A wide-spectrum coordination model of schizophrenia}

\section{Hendrik Pieter Barendregt}

Nijmeegse Instituut voor Informatica en Informatiekunde, 6500 GL Nijmegen, The Netherlands. henk@cs.kun.nl http://www.cs.kun.nl/ henk

\begin{abstract}
The target article presents a model for schizophrenia extending four levels of abstraction: molecules, cells, cognition, and syndrome. An important notion in the model is that of coordination, applicable to both the level of cells and of cognition. The molecular level provides an "implementation" of the coordination at the cellular level, which in turn underlies the coordination at the cognitive level, giving rise to the clinical symptoms.
\end{abstract}

The model of schizophrenia presented by Phillips \& Silverstein (P\&S) can be depicted as follows:

$$
\text { NMDA } \downarrow \Rightarrow \text { neur. coord. } \downarrow \Rightarrow \text { cogn. coord. } \downarrow \Rightarrow \text { schiz. } \uparrow
$$

This requires some explanation from the following dictionary:

$\begin{array}{ll}\text { NMDA } & \text { N-methyl-D-aspertate glutamate receptor activity } \\ \text { neur. coord. } & \text { neuronal coordination } \\ \text { cogn. coord. } & \text { cognitive coordination } \\ \text { schiz. } & \text { schizophrenia symptoms } \\ X \downarrow & X \text { decreases } \\ X \uparrow & X \text { increases }\end{array}$

In somewhat more detailed terms, the model states the following. If the activity of NMDA glutamate receptors in the cortex is below normal, then neural coordination within and between cortical regions is decreased; this in turn implies decreased cognitive coordination, such as disambiguation and dynamic grouping; this then will be the direct cause of the symptoms of schizophrenia, such as impairments of perception, preattentive sensory gating, selective attention, working memory, and long-term memory. The authors choose to focus on disorganization.

The way the authors come to their model is as follows: NMDAantagonists cause schizophrenia-like symptoms; schizophrenia implies impaired cognitive coordination, and vice versa; neural coordination is behind cognitive coordination. The model postulates that the NMDA glutamate channels provide a control for the neural coordination. The rationale behind this is that the NMDAreceptors are voltage-gated, that is, they depend on both the ligand and the right voltage to be opened. So they may indeed be used to coordinate processes (they essentially have the function of an AND-gate in a computer).

One virtue of the model is that it is wide-spectrum. It ranges from a molecular mechanism via cellular phenomena, via cognition, to psychiatric symptoms. The model makes predictions about patients suffering from schizophrenia: There is impairment of global, but not local, motion perception; high frequency rhythms (gamma) will be reduced. This implies that the model is falsifiable.

The main virtue of $P \& S$ 's model is its emphasis on coordination, interaction. In computer science, a notion and theory has emerged that seems relevant here: that is, the notion and theory of communicating systems (see Milner 1999). Although everything happening in a computer may be described by fluctuating bits, the theory of communication forms a convenient level of abstraction. Some bits encode meaningful information to be used later, other bits represent actions that are relevant at the very moment. An interacting communication, the most fundamental concept in the mentioned theory, needs two half-acts, each waiting for the other half to be present simultaneously (like two persons who want to shake hands). ${ }^{1}$ All this may be useful for a thorough theoretical underpinning of the way in which coordination is implemented by NMDA glutamate channels.

Although a single model for schizophrenia is presented, this does not imply that it is a homogeneous condition. For, there are many ways in which coordination can be impaired. Also, the effects can vary in severity. The authors give several examples of this and it is also apparent from the computer science theory of communicating systems, mentioned above.

The authors mention how their model is similar to many other theories, though not in all aspects. The theories they put forward regarding the cause of the disconnection between cortical regions are mainly similar to each other, apart from the fact that they do not speak about coordination within regions and focus on long term, that is, learning, effects (see Dolan et al. 1999; Friston 1999). $\mathrm{P} \& S$ do focus in their model on the cortex but mention that other brain regions will also be involved. A paper not mentioned by the authors, in which such an involvement is described, is van Hoof (2002). Van Hoof provides a model of the pathogenesis of schizophrenia, in which the drive and guidance mechanisms in the brain (specific brain regions are mentioned) are said to be underdeveloped (in the terminology of the target article, they do not coordinate well). Such intentional aspects fit well with the model of P\&S.

The target article ends by stating many open questions. Yet, one puzzle that has been ignored is the claim in Menninger et al. (1963) that some of the schizophrenic patients get "weller than well."

The theory of mobile systems (also see Milner 1999) goes beyond that of communicating ones. The intended model in ICT (Information and Communication Technology) is that of mobile telephones, or Web pages with links. Here, the number of action channels is variable and a communication may create a new channel between other processes. This theory may model very well the way in which cells communicate. In some cases, there is no receptor in a cell for a certain transmitter $T$, but there is for another transmitter $T^{\prime}$. Reception of $T^{\prime}$ will cause the DNA code for the receptor for $T$ to be read from the genome, and brought to expression, so that $T$ can be received.

\section{NOTES}

1. A typical example of a communicating process is a vending machine. It has a slot for coins and one button for coffee and one for tea. The process of the machine is:

$$
\mathrm{M}=\text { want_coin.(ready_tea-button + ready_coffee-button).M. }
$$

This means that the machine $(\mathbf{M})$ is waiting for a coin and, after that, for a push on either the tea or the coffee button. Here, the period (.) stands for sequential composition and the $(+)$ for choice. The $\mathrm{M}$ is repeated on the right-hand side because we'd like the machine to keep operating. A human that regularly wants to use the machine has the process:

$$
\mathrm{H}=\text { put_coin.(push_tea-button + push_coffee-button).H. }
$$

Now, the interaction of the human $(\mathrm{H})$ with the machine $(\mathbf{M})$ is denoted by $\mathrm{H} \| \mathrm{M}$. Provided that we postulate that there are communications $c$, such that:

$$
\mathrm{c}(\text { put_coin,want_coin })=\text { accept_coin }
$$

$\mathrm{c}($ push_tea-button,ready_tea-button $)=$ pour_tea $\mathrm{c}$ (push_coffee-button,ready_coffee-button) = pour_coffee 
and that noncommunicating processes (like c[push_coffee-button,ready_ tea-button] are abstracted away, we obtain:

$$
\mathrm{H} \| \mathrm{M}=\text { accept_coin.(pour_tea + pour_coffee).(H\|M) }
$$

This is indeed the outlook on the world from the point of view of such a vending machine (we left out considerations that the machines need to be refilled, and that water and energy are available in unlimited quantities). We see the difference with ordinary algorithmic programming, which is directed towards termination. Programming a process is often directed in an interactive environment to unlimited continuation.

The theory of communicating systems carefully describes processes with a global control, versus ones with a local distributed control without global knowledge.

\section{Context rules}

\section{Steven L. Bressler}

Center for Complex Systems and Brain Sciences, Florida Atlantic University, Boca Raton, FL 33431. bressler@fau.edu

http://www.ccs.fau.edu/ bressler

Abstract: It is proposed that cortical activity is normally coordinated across synaptically connected areas and that this coordination supports cognitive coherence relations. This view is consistent with the NMDAhypoactivity hypothesis of the target article in regarding disorganization symptoms in schizophrenia as arising from disruption of normal interareal coordination. This disruption may produce abnormal contextual effects in the cortex that lead to anomalous cognitive coherence relations.

The human brain is an engineering marvel. Its range of capabilities far surpasses that of any animal or machine. Understanding the factors that give the human brain its unique cognitive abilities is of central importance to numerous human endeavors. Awareness has been growing in recent years that a major factor determining the brain's computational power is its connectional complexity (Stone \& Kotter 2002). While it is commonly agreed that cortical areas are specialized for processing different types of information, relatively little attention has been given to the dependence of this specialization on the connectional architecture of the cortex. A major determinant for an area's ability to process a certain type of information is the inputs that it receives. Yet, the connections between areas appear to be overwhelmingly supported by bidirectional pathways, implying that, through recursive interactions, an area's inputs from other areas will be affected by the output signals that it sends to them. Therefore, it would seem that the unique processing that is characteristic of each cortical area must be defined in terms of its interactions with other areas. It is therefore necessary, in seeking to determine the function of a cortical area, to consider the collection of areas with which it is connected, and with which it may jointly process information. This collection has been called an area's "connection set" (Bressler 2002) or "connectional fingerprint" (Passingham et al. 2002).

The anatomical pathways linking the areas of a connection set are undoubtedly crucial for defining what inter-areal interactions are possible, but the specific interactions that occur will ultimately depend on the dynamics of inter-areal coordination (Bressler \& Kelso 2001). Phillips \& Silverstein (P\&S) are amply justified in addressing the basic question of how specialized cortical processes are coordinated (Varela et al. 2001). They rightly stress the importance of dynamic coordination in visual perception (Bressler 1996) and its possible disruption as a determinant of schizophrenia (Bressler 2003). Moreover, they correctly assess the importance of coordination for the issue of local contextual effects within cortical areas (Bressler 1999; 2002).

$\mathrm{P} \& \mathrm{~S}$ are on weaker ground, however, when they attempt to formulate a general principle of cortical function from the distinction between primary and contextual influences. To define the "primary input" to cortical neurons as arising from their receptive fields, as P\&S do, is a decision fraught with difficulties. The con- cept of receptive field cannot serve as a sound basis for deriving a universal computational property of cortical neurons. Neurons in non-sensory cortical areas do not have unambiguous receptive fields, and neurons in higher-level sensory areas have large receptive fields that derive from multiple converging inputs rather than clearly defined primary inputs. In short, cortical areas with a clearly defined primary input pathway are the exception rather than the rule.

The overall lack of primary inputs should not, however, be taken to denigrate the role of local context in cortical processing. In a broad sense, all inputs to a cortical area may be considered as contextual - even those primary inputs that can obviously be defined as directly originating in the periphery. Therefore, contextual influence may be seen as a common outcome of cortical function, a property that emerges from the coordinating interactions in which a cortical area engages with the other areas of its connection set. Included within the various types of coordinating interactions may be top-down effects from high-level areas (connectionally far from the periphery), as well as bottom-up effects from low-level areas (connectionally near the periphery).

From this perspective, the interactions that a cortical area undergoes in conjunction with the members of its connection set, automatically provide context for that area's local processing (Bressler \& Kelso 2001). An understanding of the rules that govern the contextual influences exerted by cortical areas on one another may come from the study of cognitive coherence (Thagard 2000). If we assume that cognitive domains are spatially mapped in the cortex, then the dynamic coordination of cortical areas, constrained by the cortical connectional architecture, may instantiate cognitive coherence relations. In this interpretation, cognitive state depends on interacting cortical areas, which normally reach a consensus that resolves cognitive coherence and incoherence relations among participating cognitive domains. Large-scale networks of coordinated cortical areas that emerge during cognitive processing consequently reflect the recruitment and exclusion of areas according to the satisfaction of these relations. Areas that are able to express mutually consistent information are included in these networks, thereby satisfying coherence relations (positive constraint). Conversely, areas that would express information that is inconsistent with any of the included areas are excluded from participation, thereby satisfying incoherence relations (negative constraint). This viewpoint is consistent with that of $P \& S$ when they assign a functional role to cognitive coordination in schemata conflict resolution.

A prediction from this perspective is that cognitive dysfunction of the type presented by the disorganization syndrome in schizophrenia reflects an underlying discoordination of cortical areas (Bressler 2003). This interpretation is consistent with the NMDAhypoactivity hypothesis proposed by $\mathrm{P} \& \mathrm{~S}$ if one assumes, as they do, that inter-areal constraints are mediated by NMDA synapses. In neural terms, inter-areal discoordination would mean that cortical areas were unable to maintain a proper balance between engagement in and disengagement from large-scale coordinated networks (Bressler \& Kelso 2001). In terms of cognitive coherence, discoordination would be expected to result in cognitive states marked by a breakdown of coherent relations and the manifestation of incoherent ones. The disruption of coordination between areas that normally would be coordinated might appear phenomenologically as a failure to make correct associations among sensory fragments, percepts, events, or concepts, depending on the areas involved. The coordination of areas expressing inconsistent information could result in erroneous associations among those same entities. Therefore, discoordination could produce both degradative and illusory symptoms in schizophrenia. These predicted effects would not involve a malfunction of the activity within any cortical area, so they could not be detected by recording the activity of any single neuron or single area. Rather, they would have to be detected as departures from normal patterns of coordination, reflecting violations of the normal rules of context. 


\section{Spatial integration in perception and cognition: An empirical approach to the pathophysiology of schizophrenia}

\section{Yue Chen}

Mailman Research Center, Harvard Medical School/McLean Hospital, Belmont, MA 02478. ychen@wjh.harvard.edu

\begin{abstract}
Evidence for a dysfunction in cognitive coordination in schizophrenia is emerging, but it is not specific enough to prove (or disprove) this long-standing hypothesis. Many aspects of the external world are spatially mapped in the brain. A comprehensive internal representation relies on integration of information across space. Focus on spatial integration in the perceptual and cognitive processes will generate empirical data that shed light on the pathophysiology of schizophrenia.
\end{abstract}

When Bleuler (1911/1961) coined the term schizophrenia, few realized how difficult it would be to unravel the brain mechanisms underlying this devastating mental disorder. Bleuler's view of "split mind" as a basis of schizophrenia is sensible but nevertheless intricate for empirical assessment, at least up to now. Several theories have been developed to address issues of functional impairments of the brain associated with schizophrenia (e.g., Beaumont \& Dimond 1973; Friston 1998). Because the search for a relationship between schizophrenia and observable organic loss in the brain regions does not seem very promising, the hope of using dysfunction in brain networks to understand schizophrenia has been revived. Phillips \& Silverstein's (P\&S’s) perspective on cognitive coordination in schizophrenia represents a new endeavor in this direction.

By incorporating recent advances in neurophysiology into the study of psychotic conditions and reviewing experimental data associated with schizophrenic patients, P\&S conjecture that dysfunction in cognitive coordination is implicated in schizophrenia. This perspective appears to be reasonable in that it is consistent with many clinical and laboratory observations reported for schizophrenic patients. On the other hand, some of these observations are also consistent with a different perspective - a dysfunction restricted to a specialized perceptual or cognitive process.

Does dysfunction of cognitive coordination play a major role in some, rather than in other, pathophysiological processes of schizophrenia? Or does dysfunction in cognitive coordination processes and in specialized cognitive processes co-exist in schizophrenia? To distinguish these two possibilities, current empirical evidence is not adequate. To further appreciate the cognitive coordination issue, we need empirical data that address not only abnormal functions in cognitive coordination but also normal functions in relevant specialized cognitive processes in schizophrenia.

Separating a deficit in cognitive coordination from a deficit in a specialized cognitive process has never been an easy task. This is because many cognitive functions involve both specialized and coordinated processes and, as a consequence, the two types of deficits sometime show similar characteristics in behavioral responses. One aspect of cognitive function - spatial integration is, however, distinctive in this respect. Information from the external world is represented largely point-to-point in the perceptual and cognitive systems. Take vision as an example: Images of visual fields are retinotopically mapped to the striate cortex in primates. In order for the visual brain to form a coherent percept, information from different spatial locations must be integrated. This representation principle used by the brain presents a unique opportunity to differentiate the two types of dysfunctions - cognitive coordination versus functioning of a specialized cognitive process - that are potentially linked to schizophrenia. By assessing the processing of spatially localized information versus spatially distributed information, we can, in principle, dissociate the coordination of cognitive processes from the functioning of specialized cognitive processes in schizophrenia (Holzman 1994).

Perceptual organization, a visual process requiring cognitive co- ordination, has been explored in schizophrenia. The results remain inconclusive as far as whether a grouping dysfunction exists (Rief 1991). On the one hand, Place and Gilmore (1980) showed that, when asked to report the number of lines displayed heterogeneously, a task that is performed more efficiently without grouping, schizophrenic patients performed better than normal subjects, suggesting deficient perceptual organizing ability. On the other hand, Chey and Holzman (1997) showed that schizophrenic patients performed as well as normal controls did on tasks requiring the application of Gestalt principles of organization (i.e., proximity, collinearity, or similarity). A number of other studies showed results either consistent or inconsistent with a dysfunctional perceptual grouping in schizophrenia (e.g., John \& Hemsley 1992; Silverstein et al. 2000). These apparent inconsistencies may be due to schizophrenic patients' reduced but not diminished ability to combine information into a coherent whole, as pointed out by $\mathrm{P} \& \mathrm{~S}$. Before attributing these results to a dysfunction in cognitive coordination, it is necessary to assess whether the processing of other elementary information - for example, spatially localized information - is normal in schizophrenia. This critical assessment is often missing from many studies. Without such an assessment, the demonstrated deficits in schizophrenia cannot be attributed solely to a dysfunction in perceptual grouping.

One example of incomplete separation of a specialized versus a coordination deficit can be seen in the study of global and local motion processing in schizophrenia (Chen et al. 2003; cited by $P \& S)$. This study aimed to identify the stage of motion processing, the global or the local, responsible for the deficient behavioral manifestation shown in previous studies (e.g., Chen et al. 1999a; 1999b), and found that detection of coherent motion embedded in a random-dot field, but not detection of motion embedded in gratings, was deficient in schizophrenic patients. Because only detection of coherent motion requires spatial integration of motion signals, the results were taken as evidence for a deficit in global motion processing (or dynamic grouping, as stated by $\mathrm{P} \& \mathrm{~S}$ ). The detection of coherent motion also involves rejection of noise; whether this nonintegration component contributes to the performance of the patients remains unclear. Thus, further empirical studies are needed to differentiate whether or not the deficit shown in the patients is due mainly to a failure in spatial integration, rather than in noise rejection. The same reasoning can be applied to the contour integration study in schizophrenia (Silverstein et al. 2000; also cited in the target article). That study showed that schizophrenic patients were less able to detect the contours that are composed of Gabor elements. Again, contour detection in that stimulus configuration also involves rejection of Gabor elements that do not belong to contours (distractors or noise). To be qualified as evidence for dysfunction in cognitive coordination, empirical studies need to show functional integrity of other cognitive processes, including filtering out noise and encoding spatially localized visual information (such as single dot, in the case of detection of coherent motion, or single Gabor element, in the case of contour detection).

The concept of cognitive coordination, put forward by $\mathrm{P} \& \mathrm{~S}$, emphasizes the importance of the interaction among different cognitive processes, rather than the integrity of individual cognitive processes. One empirical approach to address the issue of cognitive coordination is to study spatial integration in relation to spatial structure of the perceptual and cognitive systems. Take vision again as an example. One aspect of spatial interaction in the visual system can be described as the effect of visual stimulation in the surround on the responses to visual stimulation in the center. This effect has been shown at both the neuronal and the psychophysical levels (e.g., Allman et al. 1985; Born 2000; Xing \& Heeger 2000). One advantage of this center-surround paradigm is that there is no involvement of noise or distractor in the visual stimuli. Application of this paradigm will allow spatial integration of visual information to be isolated from specialized sensory encoding and allow assessment of how visual information at different spatial locations interact with each other. The outcomes of this type of study 
Commentary/Phillips \& Silverstein: Convergence of perspectives on cognitive coordination in schizophrenia

will then shed new light on cognitive coordination in schizophrenia.

\section{ACKNOWLEDGMENTS}

I thank Dr. Philip S. Holzman and Ms. Cinnamon Bidwell for helpful comments on the early version of the commentary.

\section{Mechanisms of disrupted language comprehension in schizophrenia}

\author{
Ruth Condray and Stuart R. Steinhauer \\ Department of Psychiatry, Western Psychiatric Institute and Clinic, University \\ of Pittsburgh School of Medicine, Pittsburgh, PA 15213. \\ condrayr@msx.upmc.edus sthauer@pitt.edu \\ www.wpic.pitt.edu/research/biometrics
}

\begin{abstract}
Mechanisms that contribute to perceptual processing dysfunction in schizophrenia were examined by Phillips \& Silverstein, and formulated as involving disruptions in both local and higher-level coordination of signals. We agree that dysfunction in the coordination of cognitive functions (disconnection) is also indicated for many of the linguistic processing deficits documented for schizophrenia. We suggest, however, that it may be necessary to add a timing mechanism to the theoretical account.
\end{abstract}

The notion that aberrations in sensory-perceptual and attentional processing contribute to higher-order cognitive dysfunction in schizophrenia was apparent to the first clinicians that studied the disorder. Development of behavioral and neurophysiological methodologies in the past four decades has provided neurobiological links to those observations. Phillips \& Silverstein (P\&S) provide a careful and compelling integration of such studies, beginning with the experimental evidence indicating consistent difficulties in perceptual grouping and organization which cannot be explained by inattention alone.

$\mathrm{P} \& \mathrm{~S}$ describe the interference of perceptual discriminations that is indicative of failures of Gestalt organization. We agree that similar integrative and organization failures may contribute to schizophrenia patients' deficits in language comprehension, and that the types of rhythmic activity (i.e., gamma band oscillations) that P\&S emphasize as relevant for primary perceptual integration are likely to be significant for language function. We emphasize, however, the importance of a timing mechanism for any theoretical account of language dysfunction in this disorder. We will direct our comments to the relevance of both mechanisms (cognitive coordination and temporal processing) for language function in schizophrenia.

First, our data on receptive syntax processes in schizophrenia (Condray et al. 2002) are consistent with a formulation of the type advocated by P\&S. Compared with controls, patients exhibited reduced accuracy (i.e., not knowing who did what to whom) about object-relative sentences ("The senator that the reporter attacked admitted the error."). More important, receptive syntax and general intelligence were correlated in controls; these functions were not associated in patients. Recent additional analyses of those data illustrate P\&S's argument regarding the failure of higher-order coordination of functions that may be more locally specialized. An initial multiple regression analysis determined comprehension accuracy was predicted by a model that included the variables temporal processing accuracy (intelligibility of rapid speech) and a diagnosis $x$ semantic knowledge (WAIS-R Vocabulary subtest score) interaction term [Model: $\mathrm{R}^{2}=.32$, Adjusted $\mathrm{R}^{2}=.30, \mathrm{~F}_{2,50}=11.88, \mathrm{p}<.001$. Predictors: temporal processing (t-test $=3.21, \mathrm{p}=.002)$; diagnosis $x$ semantic knowledge $(\mathrm{t}$ test $=2.94, \mathrm{p}<.01)]$.

Table 1 presents the results of the separate regression analyses conducted for each group to increase understanding about the significant interaction. Findings show different patterns of association for the two groups: For patients, temporal processing pre-
Table 1 (Condray \& Steinhauer). Summary of separate multiple regression analyses for variables predicting comprehension accuracy for schizophrenia patients and normal controls

\begin{tabular}{|c|c|c|c|c|c|}
\hline $\mathrm{R}^{2}$ Adj. & $\mathrm{R}^{2}$ F-ratio & df & $\mathrm{p}$ beta & SE beta & $t$ test $\mathrm{p}$ \\
\hline Patients $(n=32)$ & .22 & .16 & 4.02 & 2.29 & .03 \\
\hline \multicolumn{6}{|l|}{ Variables } \\
\hline Temporal Processing & .64 & .25 & 2.63 & .014 & \\
\hline Semantic Knowledge & .03 & .02 & 1.06 & .30 & \\
\hline Controls $(n=21)$ & .59 & .54 & 12.92 & 2,18 & $<.001$ \\
\hline \multicolumn{6}{|l|}{ Variables } \\
\hline Temporal Processing & .92 & .46 & 1.99 & .061 & \\
\hline Semantic Knowledge & .10 & .03 & 4.13 & .001 & \\
\hline
\end{tabular}

dicted comprehension accuracy, but semantic knowledge did not; for controls, the reverse was true. Overall, the cumulative patterns obtained for patients' receptive syntax performance are generally consistent with $\mathrm{P} \& S$ 's assumption of a failure to coordinate cortical activity within and between cognitive sub-systems. These data suggest the additional importance of temporal processing for patients' language comprehension.

As a second consideration, we suggest that inclusion of a timing mechanism in theoretical accounts is necessary to explain the full range of language dysfunction in this disorder. Deficits in time-dependent processing as a core feature of schizophrenia have been pursued as an independent line of investigation (for a review of the early literature, see Braff et al. 1991). Findings indicate that schizophrenia is associated with disturbances in the processing of sequential, rapidly presented stimuli, including the disruptions in auditory sensory gating and visual backward masking discussed in the target article. Recognizing that this disturbance may be more complex than a mere slow processing speed, Braff and colleagues suggested that more refined distinctions are necessary, such as Breitmeyer's transient/sustained neural channel model (Breitmeyer \& Ganz 1976). That model is based on the parallel and complementary pathways of the visual system, with functional distinctions made on the basis of temporal latency, and temporal and spatial resolution. Backward masking effects are assumed to be a result of the interruption of the slower responding of the sustained channels to the target stimulus by the faster responding of the transient channels to the mask. One hypothesis is that the visual backward masking deficit in schizophrenia is due to an overreactive transient channel that compromises sustained channel function (Green et al. 1994).

Most of the experimental tasks described by $\mathrm{P} \& S$ involve rapid, sequential presentation of stimuli, but it is not clear whether they subsume a dysfunction of timing under their cognitive coordination mechanism. In combination with our receptive syntax data, considerations regarding semantic memory deficit in schizophrenia include the possibility that some type of timing dysfunction is a key mechanism. In particular, compromised semantic memory, as indexed by semantic priming deficits, may be due to dysfunction in the temporal dynamics of neural channel activation and synchronization. Recent visual backward masking studies have demonstrated that disruptions to patients' perception of rapid, sequential bits of information (single letters) represent a robust phenomenon (Butler et al. 2003; Cadenhead et al. 1997; Green et al. 1999). It is not known, however, if visual backward masking deficit can account for semantic priming disturbance in schizophrenia. Alternatively, it is possible that a temporal processing disturbance alone is sufficient to explain semantic priming dysfunction in this population. This latter type of account has been advanced for theories of dyslexia ("dyschronia": Llinas 1993; cf. the "cognitive dysmetria" for schizophrenia proposed by An- 
dreasen et al. 1998). Moreover, researchers pursuing both lines of investigation (dyslexia and schizophrenia) have suggested that processing of rapid, sequential information produces cortical oscillations in the gamma range. Thus, converging lines of inquiry and discussion include emphases on temporal processing and on binding and coherence activity that may be reflected by high-frequency cortical oscillations.

Physiologically, coherent activity of disparate brain regions must occur to process relationships among stimuli. High-frequency electrocortical oscillations in the gamma range $(30-50 \mathrm{~Hz})$ have been proposed as one of the key types of binding processes. Pulvermuller (1999) has proposed the importance of this type of activity for semantic memory formation and lexical access. John (2001) has emphasized that electrocortical binding of functions, based on gamma activity and other key oscillatory frequencies, appears to progress from patterns of coherent activity across brain regions to states where there is zero lag in onset of activity in different regions. He refers to this process as resonance. Thus, development and learning may underlie the progression to resonance. As noted by $\mathrm{P} \& S$, schizophrenia may involve a neurodevelopmental pathogenesis (Marenco \& Weinberger 2000). To the extent that brain organization is disrupted during crucial developmental periods, such as the migration of cortical neurons during prenatal development and the synaptic pruning during adolescence, the likelihood of interference in the progression toward resonance would therefore be increased.

We agree with the authors that the normal pattern of interconnectivity among cognitive functions is disrupted in schizophrenia, with some type of disconnection account potentially explaining a range of language disturbances for this population. We wish to emphasize, however, the importance of adding a timing mechanism to theoretical accounts of language dysfunction in schizophrenia.

\section{ACKNOWLEDGMENTS}

This work was supported by the National Institute of Mental Health (MH 50631; MH 55762), and with resources and the use of facilities at the VA Pittsburgh Healthcare System, Highland Drive Division.

\section{Setting domain boundaries for convergence of biological and psychological perspectives on cognitive coordination in schizophrenia}

\section{J. P. Ginsberg \\ Neuropsychology Services, 6 Viking Court, Columbia, SC 29229-3330. jpg9999@juno.com}

http://www.med.sc.edu:96/Neuroscience/Neurosci.htm

\begin{abstract}
The claim that the disorganized subtype of schizophrenia results from glutamate hypofunction is enhanced by consideration of current subtypology of schizophrenia, symptom definition, interdependence of neurotransmitters, and the nature of the data needed to support the hypothesis. Careful specification clarifies the clinical reality of disorganization as a feature of schizophrenia and increases the utility of the subtype.
\end{abstract}

The authors make clear at the outset that they are primarily concerned with the "disorganization syndrome" of schizophrenia. More should be said, then, about how the disorganization syndrome fits into the bigger clinical picture of this heterogeneous brain disorder.

Subtyping schizophrenia. It is fair to say that heretofore, subtyping schizophrenic disorders has not approached the degree of validity necessary to produce agreement about individual patients among professionals who are practicing in the clinical setting. For cognitive coordination, and its underlying neuropathology, to represent an isolatable subtype with clinical utility, it is necessary to examine current schizophrenic subtypology briefly, and to support a modification of its reformulation with better specification of symptoms.
Conceptualizations of subtypes of schizophrenic disorder from the 1930s to the 1990s used dichotomous categorizations: Type I/ Type II, Nondeficit/Deficit, Reactive/Process, and Positive/Negative. The first of each listed pair would be generally characterized by good premorbid function, abrupt onset with an identifiable stressor, flat affect, and fair to good prognosis; the second of the pair is characterized by a baseline of social withdrawal, insidious onset, absent stressors, affective lability, and unfavorable prognosis.

The Diagnostic and Statistic Manual-IV (DSM-IV) does not employ any dichotomous classification of schizophrenia. The Axis II, Cluster A personality disorders (Schizotypal, Schizoid, and Paranoid) comprise what was earlier designated as Simple Schizophrenia (Sanislow \& Carson 2001). Although paranoid conditions are still viewed as distinct from other psychotic disorders (Blaney 1999), they are widespread throughout the DSM-IV, falling into Cluster A, Delusional Disorder, and Paranoid Schizophrenia. The remaining DSM-IV subtypes of schizophrenia are Disorganized, Catatonic, Undifferentiated (also referred to in current literature as "Mixed"), and Residual. Disorganized thought (and behavior) are choice principle criteria that, when predominant, are sufficient to define the subtype. However, negative symptoms are not placed into classification as a single subtype, but rather are listed as one of the criteria of the choice principle, and so may be associated with any subtype.

Recent studies, in line with the target article, have now established that the dichotomous factor designated "Positive" is better divided into two factors: Psychotic (hallucinations and delusions) and Disorganization. A third factor, Negative symptoms, still emerges. (Suggestions that there is furthermore a fourth dimension - relational - are not as well supported at this time.) Awareness among the authors of the DSM-IV in 1994 evidently was great enough to spur them to include an appendix with "Alternative Dimensional Descriptors for Schizophrenia" that corresponds exactly to the three-factor solution: Psychotic (Hallucinations or Delusions), Disorganized, and Negative.

Symptom definition. The three-factor solution of schizophrenia has the diagnostic effect of separating Disorganized thought from the Psychotic symptoms in one subtype, although one may reasonably hope that this was a de facto outcome of careful observation and diagnostic acumen anyway. The importance of this insight is its etiologic implications. Following the lines of clinical correlation, it appears that Negative symptoms are still associated with the Psychotic as well as the Disorganized subtype. This raises questions about the relationships among the symptom complexes. Negative symptoms could be either a downstream effect of delusions/hallucinations and thought disorder or could be a fundamental deficit that has different outcomes. This is a question to be explored further empirically, for example by using clinical notes. Similarly, one would like to know the comorbidity rates of Negative symptoms with Disorganized and Psychotic subtypes. By the way, it proves a difficult task to find surprisingly simple demographics about the population with schizophrenia, such as the relative prevalence of subtypes. One reference notes 55\% Paranoid subtypes among successive admissions with any type of schizophrenia (Hachem et al. 1997), but a prevalence figure for the Disorganized subtype was not found.

Neurotransmitter systems. The authors are well aware that hypofunction of NMDA receptors has effects on other neurotransmitters systems, and note that dysregulation of dopamine in prefrontal cortex, resulting in a chronic decrease of utilization, is produced by NMDA-antagonists. This fact seemingly adds to the basis on which Disorganization symptoms (NMDA-hypofunction) can be separated from Negative symptoms (prefrontal dopamine decreased utilization). The dissociation (or lack of it) of these neurotransmitter system abnormalities is not directly addressed. This harks back to the need, mentioned above, for comorbidity prevalence data, to determine how often a schizophrenic Disorganization syndrome occurs with and without Negative symptoms. Clinical anecdotal perspective suggests that many patients with 
Disorganized schizophrenia have little or no trouble with Negative symptoms, and in fact can be impulsive, aggressive, and unpredictable with potential for violence. It would be important to know under what conditions prefrontal dopamine and glutamate levels do interact. The suggestion has also been made, that hypofrontal glutamate activity causes excessive mesolimbic phasic dopamine reactivity, producing psychotic symptoms (Grace 1991).

Data. The clinical research evidence linking the construct of cognitive coordination to frank thought disorder is not as strong as it needs to be to be conclusive. Disorganized symptoms were found to be associated with exposure to NMDA-antagonists in humans, but so were Psychotic symptoms (delusions and hallucinations) and Negative symptoms. Contextual disambiguation is heavily relied on as an overarching proxy for cognitive coordination, stressing the fact that both information per se, and the meaning of the signals, are embedded in context. Experimental operationalization of cognitive coordination depended heavily on the perceptual-grouping method. Although it is quite clearly indicated that perceptual-grouping scores were correlated with scores of disorganization and associative thought disturbance, there is a possible tautology in other citations which use language perception and production as indicators of cognitive coordination.

Stimulus configuration studies (Rudy \& Sutherland 1989; Sutherland \& Rudy 1989) developed a method of studying acquisition of associated stimuli in rodents (light or tone, or light plus tone). Although the original focus of interest was primarily the role of hippocampus in learning and memory, there is a connection indicated by the authors in their consideration of the influence of contextual constraints on long-term memory formation. A possible source of controlled experimental data may exist if this method were used to assess the effects of PCP-like substances on configural stimulus acquisition in rats.

ACKNOWLEDGMENT

The encouragement of E. Selman Watson, is gratefully acknowledged.

\section{Cognitive coordination deficits: A necessary but not sufficient factor in the development of schizophrenia}

\author{
Diane C. Gooding a and Jacqueline G. Braun ${ }^{\mathrm{b}}$ \\ a Departments of Psychology and Psychiatry, University of Wisconsin- \\ Madison, Madison, WI 53706; 'bepartment of Psychology, University of \\ Wisconsin-Madison, Madison, WI 53706. dgooding@facstaff.wisc.edu \\ jgbraun@facstaff.wisc.edu \\ http://psych.wisc.edu/faculty/bio/gooding.htm
}

\begin{abstract}
The Phillips \& Silverstein model of NMDA-mediated coordination deficits provides a useful heuristic for the study of schizophrenic cognition. However, the model does not specifically account for the development of schizophrenia-spectrum disorders. The P\&S model is compared to Meehl's seminal model of schizotaxia, schizotypy, and schizophrenia, as well as the model of schizophrenic cognitive dysfunction posited by McCarley and colleagues.
\end{abstract}

Since Meehl's (1962) address to the American Psychological Association, investigators have conjectured that a failure of inhibition at the cellular level is associated with a failure of inhibition at the cognitive level. In Meehl's model, a genetic diathesis produces schizotaxia, an integrative neural defect in the CNS of preschizophrenic individuals. In combination with social learning influences, the genetically determined defect of schizotaxia, gives rise to a latent personality organization known as schizotypy. According to Meehl's (1962; 1989) model, all individuals who possess schizotypy would be expected to show some evidence of aberrant information processing.

Like others (cf. McCarley et al. 1999), Phillips \& Silverstein
$(\mathrm{P} \& S)$ invoke advances in neuroscience to account for the information-processing deficits observed in schizophrenia. P\&S assert that hypoactivity in the NMDA glutamate receptor channels serves as the mechanism for cognitive dysmetria. There is considerable overlap between the $\mathrm{P} \& \mathrm{~S}$ theory of cognitive coordination and the McCarley et al. (1999) model of cognitive dysfunction. Both models discuss NMDA receptor blockage as a fundamental mechanistic factor in the underlying cognitive deficits of schizophrenia. The latter model also ties in event-related potential (ERP) findings, most notably those pertaining to the N100 component, which indicate that schizophrenia-spectrum subjects show contextual processing abnormalities. While McCarley et al. have focused primarily on temporal cortical regions, $\mathrm{P} \& S$ propose that the anatomical substrates of the cognitive coordination deficit are more global, including, but not limited to, the prefrontal cortex.

The authors' conceptualization of a failure in cognitive coordination maps on very nicely to Meehl's construct of schizotaxic cognitive slippage, which he proposed could account for the cognitive, clinical, and behavioral symptoms associated with schizophrenia. However, the cognitive impairments that characterize schizophrenia are necessary but not sufficient for the development of schizophrenia. In Meehl's model, a key aspect is an underlying genetic diathesis for the disorder. The $\mathrm{P} \& S$ model is distinctly lacking a behavioral genetics perspective. Explicitly relating a specific genetic diathesis for schizophrenia to NMDAchannel hypoactivity might link the disruptions on the cellular level not only to disruptions on the cognitive and phenomenological levels, but also to a schizophrenia-spectrum outcome.

According to Meehl, there are various outcomes for schizotaxia; these outcomes might include aberrant personality traits and/or laboratory test performance, schizophrenia-spectrum disorders such as schizotypal personality disorder and schizoaffective disorder, and schizophrenia. In the P\&S model, the ways in which schizophrenia arises from a failure in cognitive coordination are not explicated. The failure in cognitive coordination that is posited in the P\&S model accounts for various psychotic conditions, including, but not limited to, frequently observed phenocopies of schizophrenia, such as PCP-psychosis. Although P\&S compare the effects of NMDA-antagonists to the impairments observed in schizophrenia, the same impairments could be noted in non-schizophrenia patients who suffer from psychosis. Thus, the $\mathrm{P} \& S$ model appears to fall short in accounting for the ways in which the neural bases of cognitive disorganization lead specifically to schizophrenia and/or schizophrenia-spectrum disorders.

One positive aspect of the P\&S model is that it incorporates a neurodevelopmental perspective. However, one corollary of a neurodevelopmental model of schizophrenia is that signs of the underlying liability for schizophrenia should precede the manifestation of schizophrenia symptoms. Schizotypal traits are more common among first-degree relatives of schizophrenia patients (Gottesman 1991) and other individuals at increased risk for the later development of schizophrenia (Kwapil 1998). In our lab, we have observed that schizotypal individuals are more likely than nonschizotypal controls to display deficits similar to the ones observed in schizophrenia patients, namely, cognitive slippage (Gooding et al. 2001), disinhibition as measured by an anti-saccade task (Gooding 1999), increased perseverative errors on the Wisconsin Card Sorting Test (Gooding et al. 1999), and subtle working memory impairments (Tallent \& Gooding 2000), as well as smooth-pursuit eye-tracking deficits (Gooding et al. 2000). Findings that such individuals also show evidence of NMDA-receptor channel hypoactivity and/or higher levels of endogeneous NMDA-receptor blocker, N-acetyl-aspartyl-glutamate (NAAG), would buttress support for the P\&S model.

We also assert that the cognitive coordination deficit described by $P \& S$ does not adequately capture the cognitive signature of schizophrenia. Inhibition is broadly defined to include the following processes: deliberate controlled suppression of prepotent responses; decrease in activation of some nodes (as in connectionist 
models) and/or an increase in other nodes; and sensory gating or filtering (Miyake et al. 2000). Through these processes, inhibition provides interference control and assists in processing relevant information. Although inhibition and working memory are associated constructs, they are not entirely overlapping processes. While $\mathrm{P} \& \mathrm{~S}$ discuss inhibition as one aspect of cognitive coordination, they discuss inhibition primarily in terms of its service in the maintenance of context. However, the disinhibition seen in patients with schizophrenia encompasses a broader array of impaired processes. In addition to disinhibition of prepotent responses such as those seen during Stroop and anti-saccade tasks, schizophrenia patients also manifest sensorimotor gating deficits as measured through prepulse inhibition paradigms (cf. Braff et al. 1999) and perseveration in behavioral responses as well as in language production. We would therefore assert that disinhibition, in addition to failure to maintain contextual representations, is part of the cognitive signature of schizophrenia.

The P\&S model departs from that of Meehl (1962; 1989) by allowing for etiological as well as phenomenological heterogeneity. Indeed, the authors assert that their model is particularly relevant for the disorganized subtype of schizophrenia. In the final section of their paper, $\mathrm{P} \& \mathrm{~S}$ present several avenues for further theorizing and investigation. One further avenue for refining the P\&S model might be to relate other aspects of neurochemical dysregulation, for example, the effects of serotonergic hallucinogens and/or dysregulation of the hypothalamic-pituitary-adrenal (HPA) axis (cf. Walker \& DiForio 1996) to the coordinating interactions that are implemented via NMDA-receptors. Such theorizing might account for the exacerbation of at-risk individuals' cognitive impairments under conditions of stress.

In summary, the P\&S model fails to link the NMDA-mediated cognitive dysmetria specifically to the development of schizophrenia and spectrum disorders. Nonetheless, we believe that it provides a valuable heuristic for our understanding of schizophrenia and are encouraged by its potential for further research inquiry.

\section{Linking brain to mind in normal behavior and schizophrenia}

\section{Stephen Grossberg \\ Department of Cognitive and Neural Systems, Boston University, Boston, MA 02215. steve@bu.edu http://www.cns.bu.edu/Profiles/Grossberg}

Abstract: To understand schizophrenia, a linking hypothesis is needed that shows how brain mechanisms lead to behavioral functions in normals, and also how breakdowns in these mechanisms lead to behavioral symptoms of schizophrenia. Such a linking hypothesis is now available that complements the discussion offered by Phillips \& Silverstein (P\&S).

This interesting target article emphasizes the important role of "cognitive coordination" - notably contextual disambiguation and dynamic grouping - in schizophrenia, and proposes that a failure of such coordination may occur during schizophrenia as a result of reduced ion-flow through NMDA glutamate receptors. The article summarizes a variety of useful data that support this hypothesis to varying degrees. There is, however, a large gap in the authors' argument that would need to be filled for the hypothesis to be more compelling: Namely, no linking hypothesis is supplied to explain how brain mechanisms of cognitive coordination work, or how they generate contextual properties at the behavioral level in normal individuals; or how the proposed breakdown of these mechanisms leads to schizophrenic symptoms. As a result, the authors' arguments are basically arguments of consistency with some known data, rather than arguments from which a compelling deduction of cause and effect can be derived.

Because of this absence of computational rigor, some of their statements can be confusing or even misleading. For example, the authors write that "subsets of neuronal responses are grouped by synchronizing the spiking activity of which they are composed" (target article, sect. 1, para. 2). This seems to say that synchrony causes grouping, whereas it is detailed neural models of vision which show how grouping can sometimes cause synchrony (Grossberg 1976; Grossberg \& Grunewald 1997; Grossberg \& Somers 1991). A second possible cause of confusion is that mechanistically different types of cognitive coordination tend to be lumped together, rather than distinguished by their unique characteristics. For example, the authors mention working memory in the prefrontal cortex (PFC) as an example of cognitive coordination (sect. 1) but do not indicate that the circuits that control working memory in the PFC (e.g., Bradski et al. 1994; Grossberg 1978) can have different organization and properties than, say, those which govern grouping in V1 and V2 (e.g., Grossberg 1999; Grossberg \& Raizada 2000)

The authors' working hypothesis is "that cortical activity is coordinated by widely distributed local interactions within and between regions, as well as by top-down strategic commands" (sect. 1 , para. 12), a view that is presently shared by many neuroscientists. Stated so broadly, this hypothesis is hard to use effectively. In fact, detailed models of the laminar architecture of the neocortex now clarify how such bottom-up, top-down, and horizontal interactions are organized for purposes of perceptual grouping, attention, development, and learning (e.g. Grossberg 1999; Raizada \& Grossberg 2003), and would be a good foundation for testing the authors' general hypothesis. These LAMINART models have qualitatively explained and quantitatively simulated key grouping and attentional effects about visual What stream processing that the authors review; for example, in section 2.1. The authors then immediately discuss processing in the Where cortical stream of coherent object motion. Although these What and Where processes share some mechanisms, they also have computational complementary properties (Grossberg 2000b), as a result of the differences between generating orientationally sensitive groupings of object form and generating directionally sensitive groupings of object motion. Key data about coherent object motion have also been quantitatively simulated (Chey et al. 1997; Grossberg et al. 2001), and one can now study precisely how form and motion processes differ, and how they interact to overcome their complementary deficiencies.

Models have elsewhere been proposed that attempt to show how cognitive-emotional neural processes can explain normal behavioral and brain data about learning in animals and humans, and prescribed breakdowns in these models give rise to schizophrenic negative symptoms. These models include feedback interactions between brain regions such as the sensory cortex, amygdala, and prefrontal cortex, and propose how an overaroused or underaroused opponent process in the amgydala or prefrontal cortex can lead to negative symptoms (Grossberg 1984; 2000c). These models do include a possible role for DA (dopamine) dysfunction in giving rise to overaroused or underaroused condition. Given the derivation of these models from properties of cognitive-emotional learning, they may also accommodate problems with NMDAreceptors. Related models of sensory and cognitive learning suggest how tonically hyperactive volitional signals, say from the basal ganglia, could lead to positive symptoms like hallucinations (Grossberg 2000a), as a manifestation of the learned top-down expectations that normally help to stabilize learning and, along the way, focus attention on, and prime, the brain to selectively process objects of interest. 


\section{Schizophrenic cognition: Taken out of context?}

\section{David R. Hemsley}

Psychology Department, Institute of Psychiatry, Denmark Hill, London SE5 8AF, England. d.hemsley@iop.kcl.ac.uk

Abstract: This commentary addresses: (a) the problems of definition which have been prominent in the use of the term context in schizophrenia research; (b) potentially useful distinctions and links with other theories of schizophrenic cognition; and (c) possible pathways to schizophrenic symptoms. It is suggested that at least two major aspects of the operation of context may be distinguished and that both may be impaired in schizophrenia.

In this stimulating article, the authors define context in terms of its mode of operation rather than in terms of stimulus parameters. This neatly sidesteps the problem of the various ways in which context has been defined in the schizophrenia literature, ranging from task instructions to an immediately preceding simple stimulus. Phillips \& Silverstein (P\&S) are therefore dealing with what Mayes et al. (1985) refer to as interactive context. However, the characteristics of nontarget stimuli will determine whether they influence target processing. Although such factors as temporal and spatial contiguity will play a role, a crucial determinant will be the pattern of stored predictive relationships existing between target and nontarget stimuli, as emphasized by Phillips and Singer (1997a). This is similar to the operation of Broadbent's (1971) "pigeon-holing" mechanism, which acts to bias category state thresholds; a disturbance of this process in schizophrenia was proposed many years ago (Hemsley 1975).

A number of distinctions may be important to an understanding of schizophrenic dysfunctions: (1) Temporal versus spatial context. These may influence the processing of target stimuli in different ways and in many experimental situations may be difficult to disentangle. For example, the situation may be complicated by abnormalities of visual scan paths in schizophrenia (e.g., Green et al. 2000). (2) The parameters of the target/nontarget relationship may crucially affect the nature of the influence observed and the extent of any schizophrenic abnormalities (cf. Strandburg et al. 1997). (3) Context can clearly have both inhibitory and facilitatory effects (cf. Cohen et al 1996). Abnormalities in both inhibitory (e.g., Braff et al. 1999; Hemsley 1994) and facilitatory (e.g., Spitzer 1997) processes have been documented in schizophrenia, although findings on the former, employing a variety of paradigms, appear better established. (4) Linked to (2) is the issue of "tonic" versus "phasic" context. Lubow (1989) goes so far as to define context as "all of those environmental stimuli relatively constant during the course of a procedure" (p. 74). Depending on one's definition of "procedure," this could exclude many findings that have been considered relevant to contextual disturbance in schizophrenia.

The word context is derived from the Latin contexere - to weave together - and can mean both "the connection of the parts of a discourse" and, more concretely, "the parts which immediately precede or follow any particular passage of text and determine its meaning" (Standard Oxford English Dictionary). It is clear that the above definition relates specifically to language, and in fact many of the early studies of schizophrenic contextual disturbance were concerned with language behaviour (e.g., Hemsley \& Richardson 1980). However, current research is clearly not restricted to this domain, and the presumed abnormality is claimed to underlie a much wider range of behaviours and experiences characteristic of schizophrenia. Thus, Anscombe (1987) argued that "perceptions are not placed in a context of background knowledge" (p. 256). In similar vein, Hemsley (1994) suggested that it is a failure of context to activate appropriate schema to guide processing that is crucial to the emergence of some forms of delusional beliefs. For example, the application of entirely inappropriate frames of reference to sensory input could correspond to primary delusions.
Although the most straightforward link between contextual disturbance and schizophrenic symptoms is that concerning language behaviour, thought disorder is but one instance of the disorganisation that may characterise psychosis. Jensterle et al. (2000) argue that there are "two sources of constraint on our behaviour which increase its long term coherence and organization" (p. 116). First, there is "contextual priming," roughly equivalent to Shallice's (1988) "contention scheduling system." Second, a "top-down" process, dependent on goals and plans. Failure of either may lead to disorganised behaviour, but it is apparent that at times the latter has also been viewed as a contextual disturbance - for example, when context is taken to include task instructions. This well illustrates the problems of definition that exist in this area and which the target article goes some way to resolve.

A disturbance in the operation of context has even been invoked to explain the disruption in the "sense of self" that many see as characteristic of schizophrenia (cf. Hemsley 1998). In this paper (Hemsley 1998), I argued that the influence of context may correspond to the "transitive" aspects of conscious experience. In a related formulation, Epstein (2000) suggests that one component of James' (1890) "stream of thought" consists of "a fringe of dimly sensed contextual information" that controls the entry of information into awareness (Epstein 2000, p. 550). Hemsley (1998) argued that the sense of self in normal subjects in part arises from the consistent manner in which context activates stored material to integrate with sensory input; a disturbance in the system is emphasized in the model of schizophrenia put forward by Gray, Hemsley, and their colleagues (e.g., Gray et al. 1991; Hemsley et al. 1993)

To emphasise the breadth of research findings which have been subsumed under the context deficit model, it should be noted that this model has been invoked to explain schizophrenic performance on Latent Inhibition (LI), Negative Priming (NP), and PrePulse Inhibition (PPI) paradigms. Therefore, in Hemsley (1994), I suggested that all involved a weakening of contextually elicited inhibitory processes. While I acknowledged that they appear to be very different, all are heavily dependent on the integration of previously presented material with current sensory input.

It is clear that in the schizophrenia literature, a disturbance in the operation of context has been inferred from numerous paradigms. However, a study by Elvevag et al. (2000) employing a variety of tasks "did not suggest a simple unitary context deficit" (p. 885). This is perhaps not surprising: Even within the field of animal learning theory, at least four different roles for context have been proposed (Hemsley et al. 1993). It is possible that abnormalities in schizophrenia exist in several aspects of the operation of context. A possible way forward is suggested by O'Reilly and Rudy (2001). While accepting the important role of the hippocampus in "encoding conjunctions between context and stimulus elements" (p. 316), they argue that this system must operate in parallel with a contextual system which "integrates over multiple experiences to extract generalities" (p. 311). They emphasize the bidirectional influences operating, and note that "the development of cortical representation can affect the trajectory of hippocampal learning and vice versa." If the development of schizophrenic symptoms is based on circuits involving both frontal and temporal regions (cf. Friston 1998), abnormalities in both aspects of the operation of context might be expected. 


\section{NMDA-receptor hypofunction versus excessive synaptic elimination as models of schizophrenia}

\section{Ralph E. Hoffman and Thomas H. McGlashan \\ Department of Psychiatry, Yale University School of Medicine, New Haven, CT 06520.ralph.hoffman@yale.edu thomas.mcglashan@yale.edu}

Abstract: We propose that the primary cause of schizophrenia is a pathological extension of synaptic pruning involving local connectivity that unfolds ordinarily during adolescence. Computer simulations suggest that this pathology provides reasonable accounts of a range of symptoms in schizophrenia, and is consistent with recent postmortem and genetic studies. NMDA-receptors play a regulatory role in maintaining and/or eliminating cortical synapses, and therefore may play a pathophysiological role.

The target article by Phillips \& Silverstein $(\mathrm{P} \& \mathrm{~S})$ proposes that the primary lesion producing disorganization symptoms in schizophrenia is dysfunction of the NMDA-receptor. They postulate that a primary consequence of this pathophysiology is a breakdown of local coordination of cortical processes, expressed behaviorally as a disruption in contextual sensitivity of information processing

It would be surprising to us if NMDA-receptors were not somehow involved in the pathophysiology of schizophrenia. Alterations in learning and adaptation are hallmarks of this disorder. These processes rely, at least in part, on neuroplastic alterations mediated by NMDA-receptors (Contestabile 2000). However, we have some concerns.

The authors disclaim that their hypothesis about NMDA-hypofunction and context disorganization can explain the different symptoms associated with schizophrenia. This is a familiar caveat among schizophrenia researchers and scholars, the conundrum of heterogeneity. However, the authors also appear uncomfortable with this disclaimer when they mention Jansen and Faull's (1991) quip to the effect that it is "impossible to propose a model of schizophrenia without ignoring most of the data" (target article, sect. 7 , para. 1). We agree that schizophrenia seems maddeningly heterogeneous, but we also feel that to model the disorder successfully, a large number of findings need be accounted for, including the nature and course of a relatively full range symptoms, plus epidemiological, genetic, and neurobiological findings.

The theory of NMDA-hypofunction and context disorganization proposed by the authors explains some phenomenology of persons with schizophrenia. We have argued, however, that more than NMDA-hypofunction must be involved; and, specifically, that cortical connectivity is reduced neuroanatomically in schizophrenia (Hoffman \& Dobscha 1989; Hoffman \& McGlashan 1997; McGlashan \& Hoffman 2000; see also Feinberg 1982/ 1983). The most parsimonious explanation for such connectivity reductions, we propose, is that schizophrenia arises from an extension of synaptic pruning in association cortex that ordinarily unfolds during adolescence. These arguments have been based on computer simulations of neural networks showing how this neurodevelopmental abnormality produces a range of schizophreniclike abnormalities - including disruptions of context-based information processing such as described by $P \& S$ - and spurious attractor states that could produce hallucinations, delusions, and thought disorder (Hoffman \& Dobscha 1989; Hoffman \& McGlashan 1997; Siekmeier \& Hoffman 2002). We have also shown how synaptic pruning based on neural competition, when turned off just below the psychotogenic threshold, produces robust cognitive advantages (Hoffman \& McGlashan 1997; 2001; McGlashan \& Hoffman 2000). Therefore, genetic factors are predicted to push to the limit synaptic pruning in higher-level association cortex, to maximize these advantages in human populations. Schizophrenia, we postulate, will represent the small percentage of the population where genetically programmed synaptic pruning is pushed too far. The genetic predisposition to schizophrenia will be maintained across generations - even though reproductive success for persons with this disorder is greatly reduced (Kendler et al. 1993) - given the cognitive benefits afforded to the larger population.

Persons with schizophrenia are predicted to be especially vulnerable to NMDA-antagonists because these drugs may function as temporary disconnection agents in a condition where disconnection is already excessive. We do agree with the authors that the main locus of disconnection in schizophrenia is within cortical regions rather than involving distant connections between regions. Our reasoning is based on a recent monkey study demonstrating that "late" (i.e., peripubescent) neurodevelopment is characterized by a loss of local, within-module connectivity while distant connections remain unchanged (Woo et al. 1997). If schizophrenia is an extension of normal adolescent cortical pruning, then local connectivity would be most vulnerable to loss in this disorder (Lewis \& Gonzales-Burgos 2000). This prediction is consistent with our more recent neural network models of this disorder (Hoffman \& McGlashan 1997; Siekmeier \& Hoffman 2002).

Are NMDA-receptor hypofunction and excessive cortical pruning really the same thing? We think not. First, only the latter perspective readily accounts for a range of post mortem findings. One such study demonstrated increased packing density of prefrontal neurons in the postmortem tissue of persons with schizophrenia compared with controls (Selemon et al. 1995). Since there is little evidence of significant loss of neurons in the cerebral cortex in schizophrenia (Selemon \& Goldman-Rakic 1999), the most likely explanation is a loss of neuropil volume, that is, the dense entanglement of dendrites and axons surrounding neurons. Other postmortem studies more consistent with an anatomic pruning model of schizophrenia include those demonstrating reduced dendritic spines on pyramidal neurons (Garey et al. 1998; Glantz \& Lewis 2000), and reduced phosphoproteins utilized by synaptic terminals (Eastwood \& Harrison 1995; Glantz \& Lewis 1997; Karson et al. 1999). Second, a pruning model predicts that the molecular basis of schizophrenia will be found in processes that maintain the corresponding microanatomy, namely, synapses and neuropil, rather than reflecting disturbed function of a particular receptor.

At the time of this writing, only two genes have been specifically linked to schizophrenia in the published literature as far as we know - one coding for dysbindin and the other coding for neuroregulin I (Stefansson et al. 2002; Straub et al. 2002). Both proteins appear to be concentrated in synapses rather than being directly referable to a particular receptor. However, the second protein has been shown to alter expression and activation of glutamate receptors. Mutant mice heterozygous for this gene have fewer functional NMDA-receptors than wild-type mice, and show behavior overlapping with mouse models for schizophrenia that is partially reversible with clozapine, a drug used to treat schizophrenia. Moreover, there is now a growing body of literature indicating that NMDA-receptors play an important role in maintaining and/or eliminating synaptic connections (Bock \& Braun 1999; Hasbani et al. 2001). Thus, bets are on that NMDA-receptors are involved in the pathophysiology of schizophrenia, but where and how these receptors fit into the causal chain of antecedents and consequences of this enigmatic illness remains uncertain.

\section{ACKNOWLEDGMENT}

Simulation studies cited above were supported in part by NIMH grant RO1-MH50557. 


\section{Peeling the onion: NMDA dysfunction as a unifying model in schizophrenia}

Daniel C. Javitt

Nathan Kline Institute for Psychiatric Research, Orangeburg, NY 10962. javitt@nki.rfmh.org www.rfmh.org/nki

Abstract: $N$-methyl-d-aspartate receptor (NMDAR) dysfunction plays a crucial role in schizophrenia, leading to impairments in cognitive coordination. NMDAR agonists (e.g., glycine) ameliorate negative and cognitive symptoms, consistent with NMDAR models. However, not all types of cognitive coordination use NMDAR. Further, not all aspects of cognitive coordination are impaired in schizophrenia, suggesting the need for specificity in applying the cognitive coordination construct.

Although neuropsychological dysfunction in schizophrenia has been extensively characterized, the majority of studies have focused on anatomical, rather than neurophysiological, characterizations. The article by Phillips \& Silverstein (P\&S) highlights the critical but long neglected need to peel away the layers that separate overt behavior from underlying neurophysiological mechanisms, and then to build new conceptualizations of schizophrenia from the bottom up.

The pattern of neuropsychological dysfunction in schizophrenia, in general, fits poorly with predictions of dopamine models. The review by $\mathrm{P} \& \mathrm{~S}$ highlights an important alternative hypothesis wherein a single ongoing deficit in $N$-methyl-d-aspartate receptor (NMDAR) transmission could account for both the symptomatic and the neuropsychological features of the disorder. Deficits in perceptual organization are particularly difficult to shoehorn into dopamine theories of the disorder. In contrast, this type of deficit is an expected and anticipated consequence of NMDAR dysfunction. The review by $\mathrm{P} \& S$ points out an intermediate mechanism, impaired cognitive coordination that labels a class of interaction contributing to cognitive dysfunction in schizophrenia.

Basic support for the NMDAR models of schizophrenia comes from the observation that phencyclidine (PCP), ketamine, and other NMDAR antagonists induce symptoms that closely resemble negative and cognitive symptoms of schizophrenia. Moreover, the pattern of dysfunction induced by NMDA antagonists closely resembles the pattern observed in schizophrenia. For example, the AX-type Continuous Performance Task (AX-CPT) is a test designed to evaluate working memory/executive dysfunction (Co- hen \& Servan-Schreiber 1992). In this task, subjects respond to a specific sequence of letters ( $A$ followed by $X$ ) presented sequentially on a computer screen while ignoring all other sequences. We (Javitt et al. 2000) have observed, as have others (Cohen et al. 1999a), that stabilized patients with chronic schizophrenia show persistent deficits in the ability to inhibit responses following presentation. In a parallel study performed by Umbricht and coworkers (Umbricht et al. 2000), an identical pattern was observed following ketamine administration to normal volunteers (Fig. 1).

In both cases, deficits were observed only in the AX (correct detection) and BX (invalid cue) conditions. Neither persons with schizophrenia nor ketamine volunteers showed false alarms to invalid targets, indicating a selective deficit in executive processing rather than a generalized performance deficit. Further, in both cases, the magnitude of deficit was similar at short and long ISI (Interstimulus interval), indicating that deficits were a result of deficits of encoding the go/no-go contingency, rather than failure in information retention. The difference in pattern is critical since dopaminergic mechanisms, in general, are involved in information retention, whereas NMDAR mechanisms are involved in initial encoding. The pattern of dysfunction in schizophrenia, following stabilization, thus corresponds to the pattern induced by ketamine challenge in normals and the pattern predicted by basic conceptualizations of NMDAR function.

Most recently, Javitt et al. (2001) have also investigated the ability of an NMDAR agonist, glycine, to reverse AX-CPT deficits in schizophrenia (Fig. 2). In our study, AX-CPT performance was evaluated prior to and following 6 weeks of treatment with either glycine $(\mathrm{n}=5)$ or placebo $(\mathrm{n}=6)$ added to typical or atypical antipsychotics. Patients showed significant improvement in negative and cognitive symptoms during treatment with glycine but not placebo (Javitt et al. 2001). A parallel improvement in BX errors was observed in the AX-CPT. During glycine treatment, a significant reduction in BX errors was observed at both short $(p=.06)$ and long $(\mathrm{p}<.03)$ ISI. In contrast, rates of BX errors during placebo treatment were unaffected, leading to significant between-group difference in improvement at both short $(p<.05)$ and long $(p<.03)$ ISIs. The effects of glycine on AX-CPT were over and above any degree of improvement that had occurred during antipsychotic stabilization. Therefore, $P \& S$ are correct that deficits in working memory/executive processing, as measured with tests such as the AX-CPT, may reflect underlying NMDAR dysfunction.
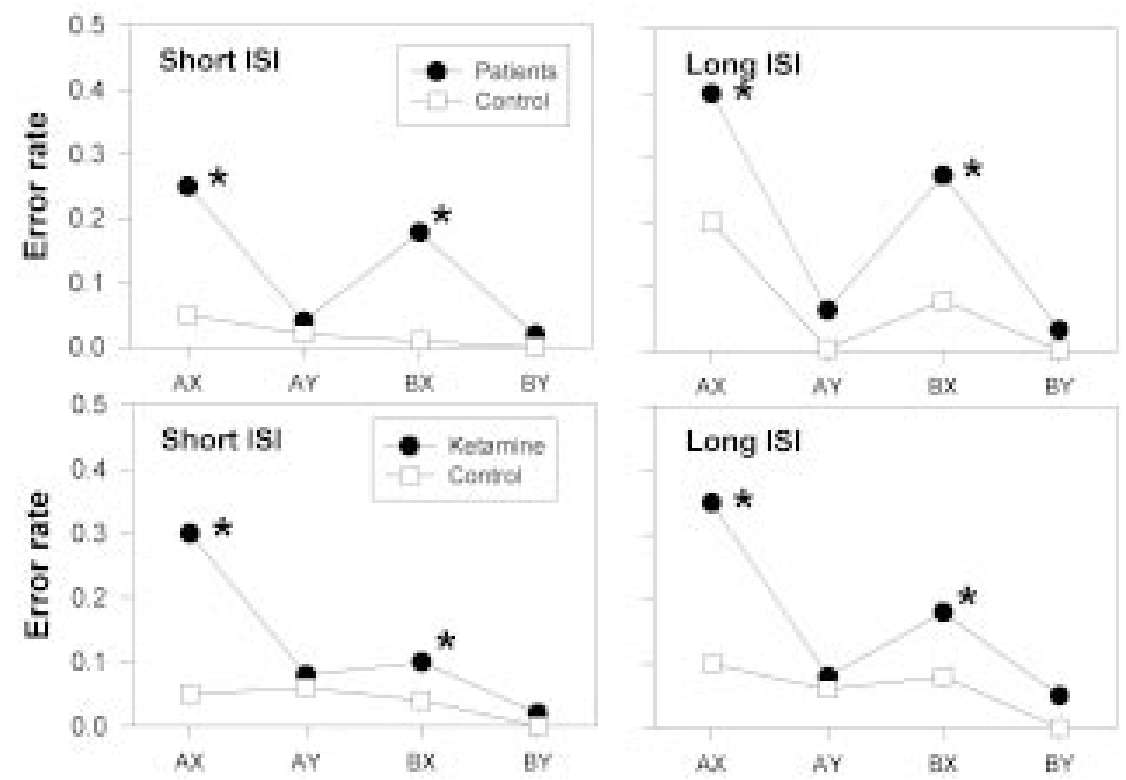

Figure 1 (Javitt). Performance of schizophrenia patients (top row) and ketamine-treated normal controls (bottom row) on the AX-type continuous performance task (AX-CPT). Note increased rates of omission (AX) errors, and increased false alarm rates only to BX-type trials in both groups. Schizophrenia data is from Javitt et al. 2000. Ketamine challenge data is from Umbricht et al. $2000 .{ }^{*} \mathrm{p}<.05$ 


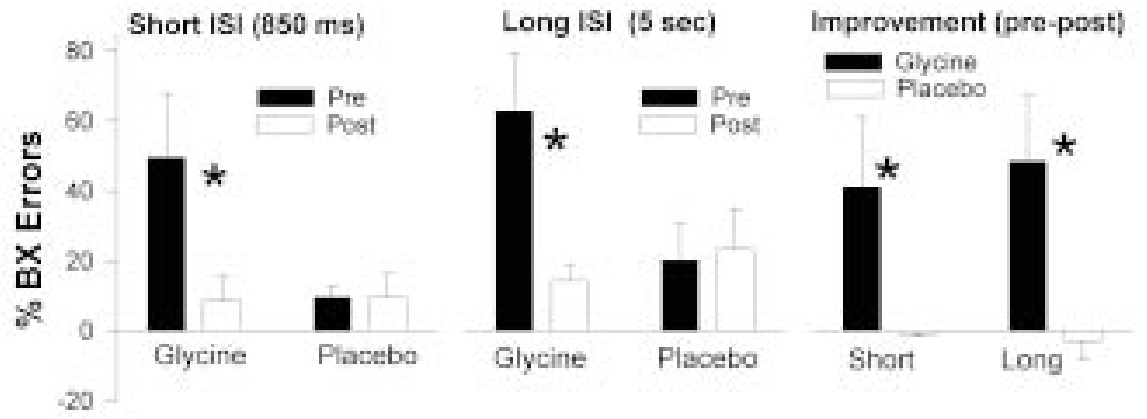

Figure 2 (Javitt). Rates of BX errors on the AX-type continuous performance task (AX-CPT) in patients with schizophrenia prior to and following treatment with glycine (an NMDA agonist) or placebo. Note decrease in BX error rate from pre- to post-treatment at both short (left panel) and long (center panel) interstimulus intervals (ISI) following treatment with glycine but not placebo. This improvement was significantly greater in the glycine, than placebo, group (right panel). ${ }^{*} \mathrm{p}<.05$

Although we agree with $P \& S$ about the role of NMDAR dysfunction in schizophrenia, we are concerned that "cognitive coordination," while broader than the previous conceptualizations such as "executive processing" or "working memory," still does not capture both the breadth and specificity of NMDAR-related phenomena in schizophrenia. For example, NMDARs play a crucial role in regulating subcortical dopamine systems (Balla et al. 2001; Kegeles et al. 2000). While this type of interaction does not fit easily within the "cognitive coordination" rubric, it is nevertheless a critical mechanism whereby a single neurochemical deficit, NMDAR dysfunction, could lead to complex dopaminergic dysfunction such as is observed in schizophrenia.

Similarly, in our studies of sensory processing, my colleagues and I have demonstrated that despite having severe bottom-up deficits, patients show minimal top-down deficits. Thus, in the auditory system, patients show elevated tone-matching thresholds but no increase in distractibility (Rabinowicz et al. 2000). Similarly, in the visual system, patients require greater detail before they can identify fragmented images but show relatively normal benefit from stimulus repetition or verbal cueing (Doniger et al. 2001). Both bottom-up and top-down elements of these tasks require cognitive coordination. Therefore, the need for coordination between brain regions, of itself, does not seem to predict which class of functions will be impaired in schizophrenia.

In the visual system, perceptual organization deficits, such as those observed by Silverstein and others, most likely arise because of impaired magnocellular input in dorsal stream visual areas (Butler et al. 2001), giving rise to impaired recurrent processing within the ventral stream object recognition areas as described by Schroeder and others (Schroeder 1995). Contrast gain within the magnocellular system is a process that explicitly depends upon NMDAR-mediated nonlinear amplification mechanisms (Kwon et al. 1992). Other aspects of cognitive coordination may or may not, depending on the specific underlying brain substrates. Current literature suggests that those aspects of cognitive coordination that depend upon NMDAR activation are impaired in schizophrenia. Those that are unaffected by NMDAR antagonists (e.g., ketamine, PCP), in contrast, appear to be unimpaired. Therefore, while the cognitive coordination construct adds to our understanding of mediating mechanisms in schizophrenia, it is NMDAR involvement that serves as the necessary and sufficient condition for predicting patterns of cognitive dysfunction in schizophrenia.

\section{Is sensory gating a form of cognitive coordination?}

\author{
Michael A. Kisley a and Deana B. Davalos ${ }^{\mathrm{b}}$ \\ a Department of Psychology, University of Colorado at Colorado Springs, \\ Colorado Springs, CO 80933-7150; ${ }^{\mathrm{b}}$ Department of Psychiatry, University of \\ Colorado Health Sciences Center, Denver, CO 80262. mkisley@uccs.edu \\ deana.davalos@uchsc.edu
}

\begin{abstract}
Neurophysiological investigations of the past two decades have consistently demonstrated a deficit in sensory gating associated with schizophrenia. Phillips \& Silverstein interpret this impairment as being consistent with cognitive coordination dysfunction. However, the physiological mechanisms that underlie sensory gating have not been shown to involve gamma-band oscillations or NMDA-receptors, both of which are critical neural elements in the cognitive coordination model.
\end{abstract}

As evidence to support a unified model of cognitive dysfunction in schizophrenia, Phillips \& Silverstein $(P \& S)$ interpret "sensory gating" abnormalities as a form of impaired cognitive coordination. To qualify as such, however, sensory gating must satisfy phenomenological and physiological requirements. It would be hard to argue that sensory gating isn't a form of cognitive coordination as delineated by $\mathrm{P} \& \mathrm{~S}$, as any sensory stimulus can modify the context of any other stimulus, provided they are proximal in space and/or time. The physiological proposition, on the other hand, is more amenable to critical evaluation.

Sensory gating has traditionally been investigated as the reduction in amplitude of middle latency auditory evoked potentials as a result of stimulus repetition. For example, Adler et al. (1982) were the first to demonstrate that wave P50 (also P1 or Pb) diminishes in size from one acoustic click to the next for healthy individuals, but not for those afflicted with schizophrenia. This was demonstrated with a "paired-click" paradigm, in which a pair of clicks (0.5-second interclick interval) was presented every $10 \mathrm{sec}$ onds. Sensory gating was quantified as the ratio of P50 amplitude evoked by the second ("test") click of the pair to that evoked by the first ("conditioning") click. The observed impairment in response-suppression, manifest as a test-response/conditioning-response ratio near 1 compared with a ratio near 0 for controls, was suggested to underlie schizophrenia patients' common complaint of an inability to ignore, or "gate out" irrelevant sensory information. The preattentive nature of this impairment has recently been supported by sensory gating measurements taken during states of sleep (Kisley et al. 2003).

Sensory gating, as measured with the paired-click paradigm, is to be distinguished from sensorimotor gating, in which a warning stimulus tends to reduce the magnitude of startle movement elicited by a loud sound ("prepulse inhibition"). Even though abnormalities in sensorimotor gating have also been reliably demonstrated in schizophrenia, the relevant neural mechanisms differ from those of P50 gating (Braff et al. 2001). Sensory gating is also 
Commentary/Phillips \& Silverstein: Convergence of perspectives on cognitive coordination in schizophrenia

to be distinguished from the preattentive processing function $(\mathrm{s})$ measured with the mismatch negativity (MMN) paradigm, the latter being associated with increased cortical activity elicited by stimulus novelty (Picton et al. 2000), as opposed to decreased activity due to stimulus redundancy.

In the terminology of $(\mathrm{P} \& S)$, the conditioning click in the traditional sensory gating paradigm modifies the salience and subsequent neural processing of the test click. This is consistent with the phenomenological constraints of cognitive coordination. However, do the neural mechanisms that mediate sensory gating involve coordinating activity of gamma-band oscillations through NMDA-receptors, as specified in the physiological portion of P\&S's cognitive coordination model?

Regarding oscillations in the gamma-band, there is substantive evidence that neuronal oscillatory activity in the $20-50 \mathrm{~Hz}$ range - which is known to be abnormal in schizophrenia patients (Kwon et al. 1999) - is involved in the generation of middle latency auditory evoked potentials (Basar et al. 1987). In the context of sensory gating, the results of a preliminary investigation by Clementz et al. (1997) involving 10 schizophrenia patients and 10 controls suggested a possible link between the magnitude of gamma-band response and P50 suppression. However, a more recent study of 20 patients and 20 controls concluded that low frequency activity $(<20 \mathrm{~Hz})$ is a more important contributor to the suppression of middle latency evoked potentials in response to repetitive stimulation (Clementz \& Blumenfeld 2001). For example, evoked potentials shown in both reports suggest that the $20-50 \mathrm{~Hz}$ oscillations evoked by the conditioning click expire before the test click occurs. Given this, it is difficult to imagine how gamma-band oscillations could serve to "coordinate" the contextual relationship between the first and second clicks.

To date, the only evidence that NMDA-channels might play a role in sensory gating comes from pharmacological studies in a rodent model. Adler et al. (1986) demonstrated that sensory gating is disrupted in rats when an NMDA-antagonist - phencyclidine (PCP) - is administered. However, a subsequent investigation concluded that this effect does not occur through NMDA-receptor blockade, but rather by an indirect pathway involving a noradrenergic mechanism (Miller et al. 1992). More recently, two studies were conducted to determine whether NMDA-channels might still be important for sensory gating in humans (Oranje et al. 2002; van Berckel et al. 1998). Both studies showed that ketamine, another NMDA antagonist, does not disrupt sensory gating as measured by the paired click paradigm. Although glutamatergic neurotransmission appears to be involved in the generation of wave P50, other neurotransmitter and receptor systems - particularly nicotinic-cholinergic - play a more central role in the suppression of this wave during the paired-click paradigm (Adler et al. 1998b).

In summary, evidence to support the claim that sensory gating is mediated by the physiological mechanisms described in the cognitive coordination model of $\mathrm{P} \& \mathrm{~S}$ is lacking. On the other hand, sensory gating deficits could still play a role in cognitive coordination dysfunction. As summarized by $P \& S$, and originally proposed by Venables (1964), the inundation that would result from an inability to filter out irrelevant sensory stimulation could lead to cognitive fragmentation. Based on this idea, a fruitful theoretical approach would be to model the mechanism by which unselected and overwhelming input signals could disrupt the subsequent formation of laterally-connected cortical networks that are central to the cognitive coordination framework.

\section{Theory of mind in schizophrenia: Damaged module or deficit in cognitive coordination?}

David Leiser and Udi Bonshtein

Department of Behavioral Sciences, Ben-Gurion University, IL 84105 Beersheva, Israel. dleiser@bgumail.bgu.ac.il http://www.bgu.ac.il/ dleiser

Abstract: Schizophrenics exhibit a deficit in theory of mind (ToM), but an intact theory of biology (ToB). One explanation is that ToM relies on an independent module that is selectively damaged. Phillips \& Silverstein's analyses suggest an alternative: ToM requires the type of coordination that is impaired in schizophrenia, whereas ToB is spared because this type of coordination is not involved.

Phillips \& Silverstein (P\&S) document how schizophrenic patients exhibit deficits that may be explained by impaired cognitive coordination. The specific phenomena they discuss involve paradigms that engage perceptual and low-level semantic processes. Can this line of analysis also account for specific deficit patterns in higher cognitive and social-cognitive functioning in schizophrenia?

We summarize here work of our own and by others on the abilities of schizophrenic patients to handle "naïve theories" in two contrasting domains: Theory of mind ( $\mathrm{ToM}$ ) and theory of biology (ToB). ToM is defined as the ability to attribute mental states to the self and to others, to predict and explain their behavior with reference to mental states (Premack \& Woodruff 1978). Several recent studies examined ToM in schizophrenia, and all indicate that ToM is damaged in the acute phase (e.g., Doody et al. 1998; Frith \& Corcoran 1996) but returns to normal in periods of remission (Drury et al. 1998). This is a specific deficit that cannot be accounted for by IQ or memory. As is well-known, a similar specific deficit is found in the case of autism, where it is also permanent (Baron-Cohen 1995). The relation between schizophrenia and autism was pointed out by Frith (1992), who speculated that there is a common cognitive failing in these two conditions: Autists never develop a ToM, whereas schizophrenics attempt to exercise a lost capability.

One way of testing ToM in schizophrenia uses a nonverbal paradigm, where subjects are presented with cartoons (Sarfati et al. 1997). Each cartoon strip contains three pictures, depicting a character performing some activity. Understanding these strips requires deriving the mental state and goals of the character. After studying the cartoon strip, subjects are presented with three additional pictures, one of which provides a suitable ending to the story. Another filler depicts a common, everyday activity, performed by the character, and the third is very similar to the last picture in the strip. Neither of them is related to the context of the mental states of the character as established by the strip. In a variant of the task which involved an absurd filler (Sarfati et al. 1999) virtually no patients selected that option. This indicates that the patients do attempt to make sense of the task, and the paradigm makes it possible to identify the compensatory strategy used by schizophrenic patients. Lacking understanding of what the character is up to, schizophrenic patients who are not disorganized tended to select the picture that is visually similar to the last picture. Disorganized patients and manic patients tend to select familiar everyday activities, regardless of their resemblance to the preceding pictures.

We replicated these findings, and complemented them with the more common set of stories used to test ToM (Frith \& Corcoran 1996). These stories involve understanding cheating and false beliefs, either "first-order beliefs," requiring distinguishing the beliefs of characters from the true state of affairs, or "second-order beliefs," about others' beliefs. These stories are read aloud, and simple drawings help the subjects follow and remember the plots. Schizophrenics performed significantly worse than the control groups (both normals and affective-disorders hospitalized patients). 
We tested the same subjects for ToB, adapting the battery designed by Johnson and Carey (1998). This battery relies on two levels of understanding biology, known to discriminate younger from older children. The lower level relies on acquired information about living beings. The higher level marks accession to the understanding of living beings as functional systems, which gives meaning to biological functions. (For example, because all organisms expand energy, they all must eat somehow, even if they have no apparent food ingesting organs.) In their study of Williams Syndrome (WS) patients, Johnson and Carey found that for all their verbal fluency, WS patients remain at the lower, childlike level of understanding.

Schizophrenic patients test normal on ToB, even during acute episodes. One explanation for this difference between ToM and ToB would be to posit a "module" or cerebral specialization for ToM (Povinelli \& Preuss 1995). The alternative, "theory-theory" view, maintains that ToM is acquired like any other naïve theory (Gopnik \& Wellman 1992). On that view, it would seem difficult to account for the dissociation of ToB and ToM.

We suggest that the challenge of ToM may be different from that encountered in other naïve theories (Leiser 2001). The tasks used to test for ToM require coordination of several pieces of information. Integration of multiple relations is a specific source of cognitive complexity (Astington et al. 2002; Halford et al. 1998; Waltz et al. 1999). In the false beliefs tasks, subjects must hold separate and coordinate the actual state of affairs, the first character's beliefs about them, and the second character's beliefs about the first one's. This coordination is evidently beyond schizophrenics. In Sarfati's paradigm tasks, selecting the right answer implies building a context for the character's actions, and this requires coordination of the successive steps. Failing this complex contextual disambiguation, subjects fall back on either familiar actions to provide meaning, or use a much reduced context, consisting of the last picture only. If this line of reasoning is correct, we would have in ToM a symptom that arises, not from a module, but from the vulnerability of ToM to a deficit in coordination. Preservation of ToB, by contrast, can be explained by the absence of such coordination once the higher level of understanding is achieved.

This conclusion remains tentative as an account of deficits specific to schizophrenia. The authors's description of the effects of "schizomimetic" drugs fit psychotic-like state in general, yet nonschizophrenic psychoses (e.g., affective psychosis) do not damage ToM to the same extent as schizophrenic psychosis (Bonshtein \& Leiser, in preparation; Sarfati et al. 1997). Equally, Sarfati et al. (1997) reported that a breakdown of schizophrenia into subtypes is unrelated to the severity of deficit in ToM, except for disorganized schizophrenia, which is associated with severe deficit in ToM. But this condition is characterized by a breakdown of personality and further traits that render cognitive collapse almost self-evident.

\section{Reconciling schizophrenic deficits in top-down and bottom-up processes: Not yet}

\section{Angus W. MacDonald \\ Department of Psychology, University of Minnesota, Minneapolis, MN 55455. angus@umn.edu}

\begin{abstract}
This commentary challenges the authors to use their computational modeling techniques to support one of their central claims: that schizophrenic deficits in bottom-up (Gestalt-type tasks) and top-down (cognitive control tasks) context processing tasks arise from the same dysfunction. Further clarification about the limits of cognitive coordination would also strengthen the hypothesis.
\end{abstract}

Phillips \& Silverstein (P\&S) put forward a hypothesis that addresses an important middle ground between a purely biological level of analysis and one that is entirely behavioral or cognitive.
Such a cognitive neuroscience approach to schizophrenia is informative because it takes advantage of constraints from each domain to build a more comprehensive (and more convincing) story about the nature of schizophrenic psychopathology. In addition to the substantive theory put forward in the target article, this is a strong example of how to build hypotheses that incorporate, rather than simply pay lip service to, multiple levels of analysis.

The authors' hypothesis is particularly ambitious in that it attempts to weave together two competing domains of psychopathological research. One tradition of research has identified abnormalities in a variety of bottom-up perceptual processes, including the Gestalt phenomena noted by the authors, as well as backward masking, mismatch negativity, and other physiological and behavioral effects associated with posterior regions of the brain. A second tradition has focused on impairments in top-down processes such as executive functioning, working memory, attentional control, and other higher cognitive processes associated with the prefrontal cortex. There is a growing body of evidence for specific deficits in both traditions of research. The authors hypothesize that the distinction between schizophrenic deficits in bottom-up holistic perceptual processes and top-down control processes is unparsimonious and misleading; both bottom-up and top-down processes are impaired by the same mechanism, NMDAhypofunction.

Science is often pushed forward by bold claims like this. For example, Cohen and Servan-Schreiber (1992) used computational modeling to demonstrate that schizophrenic impairments in attentional control and some working memory maintenance processes could be accounted for by a single mechanism (reduced gain attributable to tonic dopamine hypoactivity in prefrontal cortex). While the present authors have also developed computational models to support their claims about cognitive coordination, they have not yet taken the important steps of (1) demonstrating that the same model accounts for nontrivial, normal behavioral results in top-down control and bottom-up Gestalt tasks, both of which they refer to as "context processing" tasks, and, especially, that (2) impairments in such context processing associated with NMDA-hypofunction can reproduce impairments similar to those observed in schizophrenia patients in both domains. For example, it would be very compelling to find that the same lesion in Phillips and colleagues' models (e.g., Phillips et al. 1995) accounted for the patterns of impairment and spared performance in schizophrenia observed in both Place and Gilmore's (1980) Gestalt numerocity paradigm and in the expectancy manipulation of the AX task (MacDonald et al. 2003; Servan-Schreiber et al. 1996). Until such time, the argument that a single impairment leads to abnormal performance on both bottom-up and top-down tasks that require cognitive coordinating, is not compelling enough to reform this traditional distinction.

The task of breaking down artificial distinctions, if they exist, would be aided by greater clarity around the core construct in the hypothesis of cognitive coordination. The authors define "coordinating interactions" as "those that affect the salience or dynamic grouping of neuronal signals without changing what they mean. Such interactions do affect the interpretation of stimulus inputs, however" (sect. 1, para. 11). As it stands, the concept could be read to cover the waterfront of cognitive tasks (all of which require coordinating a task set with specific incoming stimuli). The authors have provided a number of specific examples of what they feel qualify as tasks of cognitive coordination, but it is not clear what tasks do not require cognitive coordination, or whether all tasks require this, but to varying degrees. If all tasks require cognitive coordination, the hypothesis becomes more difficult to test, as performance is confounded by other task demands generally present for cognitive tasks (including motivation, intelligence, and so on). 


\section{Context, connection, and coordination: The need to switch}

\author{
Robert D. Oades, Bernd Röpcke, and Ljubov Oknina ${ }^{1}$ \\ Clinic for Child and Adolescent Psychiatry, University of Essen, D-45147 \\ Essen, Germany. \{oades;bernd.roepcke;ljubov.oknina\}@uni-essen.de \\ http://www.biopsychology.uni-essen.de
}

Abstract: Context, connection, and coordination (CCC) describe well where the problems that apply to thought-disordered patients with schizophrenia lie. But they may be part of the experience of those with other symptom constellations. Switching is an important mechanism to allow context to be applied appropriately to changing circumstances. In some cases, NMDA-voltage modulations may be central, but gain and shift are also functions that monoaminergic systems express in CCC.

"An apple falls not far from the tree." So runs a proverb familiar to many, particularly if living in Germany. If in doubt about a diagnosis of schizophrenia, it can be helpful to ask the patient for an explanation of such a proverb. Especially, but not only, persons with a disorganised form of schizophrenia experience difficulties that may range from a shrug to profuse tangential explanations (Straube \& Oades 1992). It is reasonable, and in line with the thesis of Phillips \& Silverstein $(\mathrm{P} \& \mathrm{~S})$, to describe such problems in terms of context, connection, and coordination (CCC).

Conditioned blocking places an aspect of CCC within a laboratory setting. Normal persons experience a temporary problem with learning about the associations of a stimulus component $B$ that has just become part of a stimulus complex $A B$, where one has already started to learn about $A$. Persons with negative schizophrenic or schizotypal symptoms learn about $B$ quicker! (Bender et al. 2001; Moran et al. 2003; Oades et al. 1996). The normal interaction of events, whereby conditioning to $B$ is blocked by conditioning to $A$, is reduced in these patients. This example illustrates the contention of Hemsley et al. (1993) that such patients have difficulties in selecting current responses on the basis of stored regularities. This view is assimilated in the thesis advanced by $\mathrm{P} \& S$ here.

A change of context requires a change of coordination to effect a switch between the appropriate connections in order to mediate an adaptive response. A problem with the viewpoint of P\&S lies with the nature of the systems mediating the switches. In the case of conditioned blocking, mesocortical dopamine systems control the expression of the blocking effect (Oades et al. 1987). A parsimonious interpretation of the role of dopamine is that it facilitates switching between channels within the innervated region that controls the output that determines response (Oades 1985). Too little dopamine reduces the probability of change (perseveration, inhibit set-shift; Coull 1998), while too much jams the system (e.g., stereotypy).

However, neuroimaging studies repeatedly find marked activity related to switching elsewhere; for example, in part of the left or right intraparietal sulcus during covert or overt reversal-like shifts of attention (Beauchamp et al. 2001; Dove et al. 2000; Gurd et al. 2002). This region is not known to receive a major dopamine innervation. Indeed, these very reports also noted another patch of activity much further forward in the inferior frontal gyrus, close to one of the frontal generators of mismatch negativity (MMN: Jemel et al. 2002). The MMN is an event-related potential that has been postulated to demonstrate an involuntary switching mechanism (Näätänen 1990) and is repeatedly referred to by P\&S. (I may add that we have looked for associations of MMN with peripheral measures of dopamine metabolism in healthy and schizophrenic subjects in vain [unpublished data], and haloperidol is without effect on MMN [Kähkönen et al. 2002].)

These parietal and frontal loci of course depend much on glutamatergic and perhaps voltage-dependent NMDA-channels for the expression of their functions, even though the basis of the mechanism for a switch remains a subject for speculation. Certainly, local GABA mechanisms may inhibit/disinhibit local input and output, and some of these local circuits interacting with glutamate and dopamine are known to be dysfunctional in schizophrenia (Benes 2000a). But is it a hyper- or a hypoactive state that in these loci disrupts the glutamatergic contribution to switching in attention-related thought disorder (Shim 2002)? In the exchange of communications referred to, the question revolves around whether the inhibition of the psychiatric effects of ketamine by lamotrigine and the attenuation of glutamate release reflects downstream or upstream effects of the drug (or neither). In addition, the question is raised whether ketamine (as a model for the induction of psychosis) induces a hyperglutamatergic state (Shim) or in fact the blockade of the NMDA site induces a hypofunctional receptor that should represent the target of therapy in schizophrenia (Olney, cited in Shim 2002).

A further complication is that one of the mainstays of P\&S's thesis, the ketamine and PCP models for inducing psychotic features, is insecure. The affinities of ketamine and PCP for NMDA sites is astonishingly similar to the affinity for dopamine D2 and serotonergic 5-HT2 sites, respectively (Kapur \& Seeman 2002). The potentially nonselective effects of these substances in eliciting psychotic phenomena is disturbing for the neatly specific voltagedependent effect that forms the basis of P\&S's hypothesis. Indeed, we believe that the report in section 3 from Umbricht et al. (2000), cited in the target article to support the ketamine-glutamate model, is better viewed in these terms. The reported relationship between the psychotic-like effects of ketamine and a small MMN was based more on scores from a less widely used rather than the more commonly used rating scale, and the effect noted was but marginally more than that achieved with a serotonergic hallucinogenic agonist psilocybin.

Finally, this raises the relationship of saliency and serotonin activity, where again part of the argument of $P \& S$ relies on MMN studies. In the context of MMN and change detection care must be exerted in assuming that the saliency of a stimulus, another mainstay of the disruption of context perception in the hypothesis of $P \& S$, is crucial to change detection in schizophrenia. Although the authors relate reduced MMN to the poor ability to discriminate tone frequencies ( Javitt et al. 2000, sect. 4.2), MMN is relatively insensitive to the loudness and length of the tone (Michie 2001). Further, both Javitt and we (Oades et al. 1993) noted the strong association of MMN reduction with negative symptoms. This is not immediately consistent with the emphasis of P\&S on thought-disorder. The activity of serotonin, a transmitter that mediates gain-like effects in stimulus-processing (Reuter et al. 1997), naturally affects stimulus salience in perception (Winter et al. 1999), stimulus context in learning (latent inhibition; McDonald et al. 2003), and MMN (Ahveninen et al. 2002), and is anomalous in schizophrenia characterised by paranoid (Angelopoulos et al 2002), disorganized (Oades et al. 2000), and negative symptoms ( Jockers-Scherubl et al. 2001).

Windfalls may make a great apple pie, be poison for Dr. Spock, or represent youth following in the footsteps of their elders. It remains difficult, a priori, to determine which of these (or other) circumstances that require switches between stored regularities for an appropriate response, depends on glutamatergic or monoaminergic mechanisms. $\mathrm{P} \& \mathrm{~S}$, to their credit, recognise this and call for refinements in our understanding about monoaminergic-glutamatergic interactions in CCC.

\section{NOTES}

1. Dr. Oknina is on leave from the Institute of Higher Nervous Activity and Neurophysiology, Moscow. 
Commentary/Phillips \& Silverstein: Convergence of perspectives on cognitive coordination in schizophrenia

\section{Schizophrenia: Putting context in context}

Sohee Park, Junghee Lee, Bradley Folley, and Jejoong Kim

Department of Psychology and the Center for Integrative and Cognitive Neuroscience, Vanderbilt University, Nashville, TN 37240.

\{sohee.park; junghee.lee; brad.folley; jejoong.kim\}@vanderbilt.edu http://www.psy.vanderbilt.edu/faculty/sohee/

Abstract: Although context-processing deficits may be core features of schizophrenia, context remains a poorly defined concept. To test Phillips \& Silverstein's model, we need to operationalize context more precisely. We offer several useful ways of framing context and discuss enhancing or facilitating schizophrenic patients' performance under different contextual situations. Furthermore, creativity may be a byproduct of cognitive uncoordination.

Current neurobiological theories of schizophrenia mirror the dominant, localizationistic trends in neuroscience. Yet, after decades of "lobe-trotting" under intense scrutiny, no single region has emerged as the clear culprit. Similarly, psychological models tend to favor omnipotent single mechanisms (e.g., context processing, working memory, inhibitory control, sensory gating, etc.) to explain a bewildering range of symptoms that characterize schizophrenia. Alas, the glass slipper does not fit.

Reasonable theories of schizophrenia must address multiple cortical and subcortical interactions that can explain a wide range of symptoms under different conditions. Phillips \& Silverstein's (P\&S's) hypothesis that the disorganized syndrome is the clinical manifestation of deficits in cognitive coordination caused by NMDA-hypoactivity is a great leap forward in demystifying the heterogeneity of schizophrenia. One major contribution of the target article is a stimulating and thoughtful discussion of the concept of context. Although context-processing deficit has been proposed to be a core feature of schizophrenia (Cohen \& Servan-Schreiber 1992), it is not entirely clear what is included or excluded in this context. Extending the definitions of context presented in the target article, we will discuss several ways of framing context (see Fig. 1) and examine the hypothesis that context processing is impaired in schizophrenia.

Perceptual context. Focusing on the role of the attended versus unattended features (or stimuli) against the background is helpful in understanding the relationship between the selected target (or feature) and the background (or context). Context can, but does not necessarily, influence target processing.

1. Context represented within the target stimulus. A stimulus con- sists of multiple dimensions and features. If the task is to process one feature of the target (e.g., color), other unattended features provide the contextual environment (e.g., location, identity, size, speed, etc.). The same is true with auditory stimuli.

2. Context surrounding the target stimulus. The layout (whether visual or auditory) around the target provides contextual information and may influence the processing of the target. (e.g., perceptual grouping). Context can also influence the target processing across modalities.

3. Temporal relationship between the target event and the contextual background. The interval between the target onset and the contextual element may determine the extent of interaction. For example, lexical disambiguation depends on the temporal proximity between the target and the context. In associative conditioning, temporal contiguity is crucial.

Cognitive context. 1. Long-term memory, skills, and habits provide the context. There is an "influence of stored memories of regularities of previous input on current perception" (Gray et al. 1991). The contextual influence can be direct, explicit, and aware or indirect, implicit, and subconscious. For example, in a semantic priming task, experiences and associations play a key role in determining the speed of lexical decision. The Kamin blocking effect and latent inhibition also depend on the context.

2. Task-relevant information in working memory is identified as providing context (Cohen \& Servan-Schreiber 1992). For example, in a AX-type Continuous Performance Task (AX-CPT) task, subjects must respond to an $X$ only if it follows an $A$. In the Stroop task, the correct response depends on the inhibition of overlearned relationship in context.

Socio-affective context. A more global context is set by the affective state of the individual. The target article is focused on cognitive coordination, but affect is likely to influence all levels of perceptual and cognitive processing. Moreover, NMDA-hypoactivity or PCP-psychosis is accompanied by changes in affect. Positive affect alters a wide range of cognitive processes via moderately increasing dopaminergic action (Ashby et al.1999). Different affective states can either facilitate or reduce prefrontal functions (Gray et al. 2002).

Do schizophrenia patients have deficits in context processing? Deficits in context-processing are central to major theories of schizophrenia, but data from our laboratory suggest that context processing may depend on the context.

Perceptual context. We found that schizophrenia patients are sensitive to the context represented within the target stimulus in

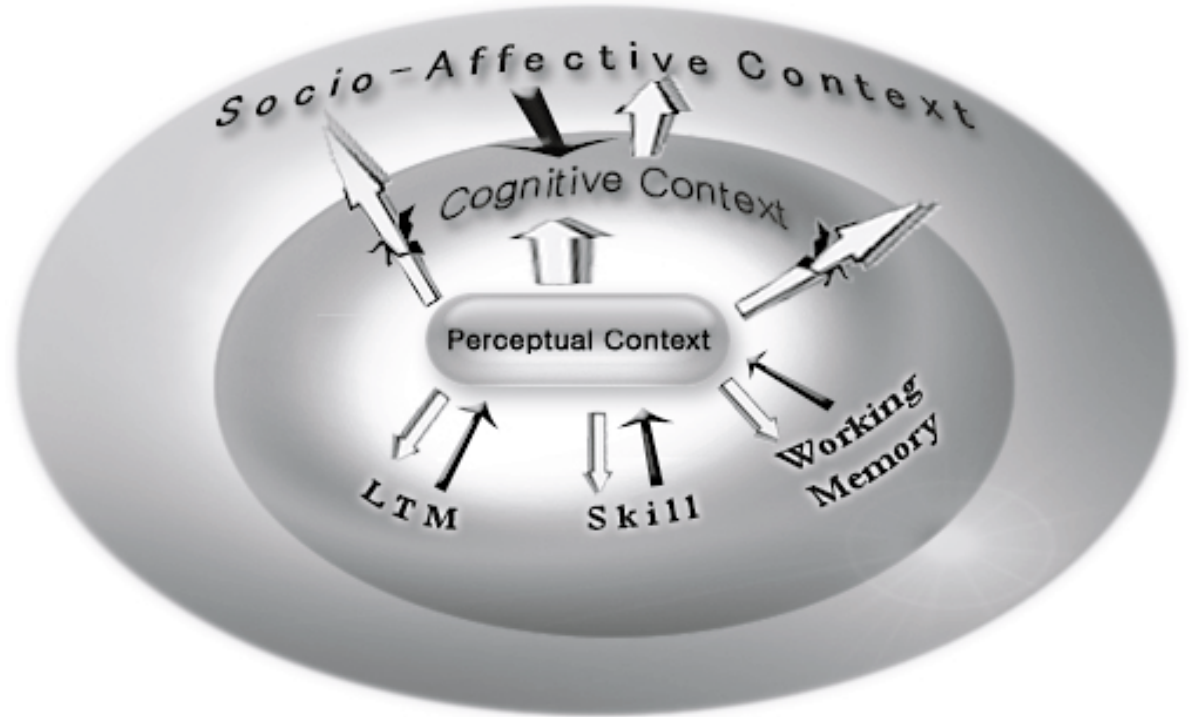

Figure 1 (Park et al.). 
a spatial working memory (SWM) task. About $80 \%$ of schizophrenia patients show profound deficits in SWM. We were able to facilitate SWM by manipulating the target type without changing the task itself. In $80 \%$ of the trials, we presented a black circle as the target. In remaining trials, we presented unusual stimuli (e.g., Korean letters or faces) and found that accuracy of schizophrenia patients on SWM was increased in these trials: Schizophrenia subjects benefited from the contextual information embedded within the target (Lee \& Park 2002a; Park et al. 2000). Schizophrenic subjects are also sensitive to the perceptual context surrounding the target.

We asked subjects to remember a random configuration of spatial locations, one of which was probed with a cue after a short delay. Performance on this task is best when the global spatial configuration of the display is preserved from the encoding to retrieval stages. Schizophrenic subjects were just as sensitive to global spatial configurations as controls. These results indicate that, at least in SWM tasks, schizophrenics may exhibit intact perceptual grouping and normal contextual processing (Lee et al. 2003a).

Cognitive context. We found that by manipulating the salience of the target to maximize the role of stimulus-driven attention at the encoding stage, we could improve AX-CPT performance in schizophrenic patients. The working memory component within the AX-CPT was manipulated by varying the interstimulus interval (ISI). Salient cue (e.g., red A) improved the performance of schizophrenia patients in AX-CPT with long ISI, relative to normal controls, but not in AX-CPT with short ISI. In other words, strengthening the context without changing the meaning of the target improved AX-CPT performance in schizophrenia. (Lee \& Park 2002b).

Socio-affective context. Our data show that SWM deficits in schizophrenic subjects can be ameliorated by manipulating socioaffective input. For example, giving positive feedback in a social context improved SWM in schizophrenic patients. SWM was also facilitated by introducing direct social interaction context prior to the memory task (Park et al. 2000).

When context is "uncoordinated." While coordination allows a conventional cognitive trajectory, lack of it may be the key to creative output. Thought disorder, which is the hallmark of disorganized syndrome, may be viewed as a creative, unconventional way of using language; however, there is little empirical evidence for the speculative relationship between creativity and madness (Prentky 1979). The concept of cognitive coordination gives us a framework for deriving concrete methodology and for explaining the relationship. Unconventional solutions may occur when coordination is fragmented or randomized, or during periods of intuition when the initial context has been temporally and semantically removed from the stimulus event (Bowers et al. 1990). The facilitation of creativity in "pathological" states may result from an inherent loosening or associative elimination of contextual relationships. Here lies a potential empirical link between madness and creativity.

In sum, we laud the target article for bringing the concepts of cognitive coordination and context into a sharp focus and thereby generating specific, testable hypotheses. Once one delves into multiple levels of context, it is possible to find pockets of intact context processing in schizophrenia. The challenge is in coordinating these performance profiles across multiple levels of context to derive a coherent model of the disorder.

\section{Inferring contextual field interactions from scalp EEG}

\author{
Mark E. Pflieger \\ Source Signal Imaging, Inc., San Diego, CA 92102. \\ mep@sourcesignal.com
}

Abstract: This commentary highlights methods for using scalp EEG to make inferences about contextual field interactions, which, in view of the target article, may be specially relevant to the study of schizophrenia. Although scalp EEG has limited spatial resolution, prior knowledge combined with experimental manipulations may be used to strengthen inferences about underlying brain processes. Both spatial and temporal context are discussed within the framework of nonlinear interactions. Finally, results from a visual contour integration EEG pilot study are summarized in view of a hypothesis that relates receptive field and contextual field processing to evoked and induced activity, respectively.

Phillips \& Silverstein (P\&S) propose that diverse impairments associated with schizophrenia may be traced to disruptions in some basic mechanisms of intraregional and interregional cortical coordination. In particular, the idealized distinction between receptive field ( $\mathrm{RF}$ ) and contextual field (CF) connections (Phillips \& Singer 1997a) may be useful, considering that CF connections function principally to coordinate multiple $\mathrm{RF}$ modules that process signals bearing primary content. Under the headings of "cortical rhythms" and "issues arising," P\&S review some relevant EEG studies, and project that EEG techniques are poised to play a role for imaging fast neuroelectric activity that reflects cognitive coordination and its impairments. This commentary discusses further considerations that arise in this context.

The RF/CF model distinguishes activity within and between cortical columns and cortical layers (see Fig. 2 of the target article). However, scalp EEG potentials (and MEG fields) are generated at each temporal instant by the net summation (weighted by sensor sensitivities) of macroscopic currents oriented throughout the gray matter volume: Currents within layers or columns cannot be distinguished directly. On the other hand, experimental manipulations combined with prior knowledge may enable some inferences about the activity of layers or columns. For example, knowledge gained from invasive studies about the local effects and mechanisms of neuromodulators (e.g., al-Amin \& Schwarzkopf 1996), particularly experiments that measure stimulus-related cortical current source density profiles as modulated by local neurochemical infusions (e.g., Javitt et al. 1996), could be combined with systemic neuropharmacological manipulations in humans (e.g. Duncan et al. 2001; Pekkone et al. 2002; van Berckel et al. 1998) to enable qualified inferences from scalp potentials about the relative contributions of activity in different cortical layers.

Potentials produced by simultaneous disjoint generators in the brain sum linearly at the scalp - a fact which enables inferences about spatial interactions in cortex based on observations of spatial nonlinearities of evoked potential responses at the scalp. If the evoked response to stimuli $A$ and $B$ presented simultaneously differs from the sum of responses to $A$ and $B$ presented separately, then the brain regions that process $A$ and $B$ interact. A prime example of this logic for making inferences about $\mathrm{CF}$ interactions based on scalp potential measurements is provided by the psychophysics-based VEP studies of Polat and colleagues (Polat \& Norcia 1996; Polat et al. 1997). Similarly, a study recently reported by Kim et al. (2002) used nonlinear interactions of partially contrast-reversing windmill-dartboard stimuli to infer that "significantly greater short-range lateral interactions and significantly less long-range lateral interactions were found in patients with schizophrenia compared to controls."

An analogous logic may be used to infer temporal interactions based on observations of temporal nonlinearities. For example, the P50 suppression effect (Boutros et al. 1995; Clementz et al. 1997 ) is a temporal nonlinearity whereby an auditory evoked component at about $50 \mathrm{msec}$ post-stimulus is modified by the precedence (within about $500 \mathrm{msec}$ ) of an identical click stimulus. In 
other words, the brain system generating the evoked response is not linearly characterized by the response to a single stimulus: Temporal context affects the evoked response to primary stimulus information. Mismatch negativity $(\mathrm{MMN})$ is another temporal context effect: Auditory cortex responds differently to the same physical stimulus as a function of its frequency or infrequency of occurrence relative to a physically different stimulus. Although both P50 and MMN may be altered in schizophrenia, MMN depends crucially on NMDA-receptors (Javitt et al. 1996), whereas P50 suppression possibly may not (van Berckel et al. 1998).

Phillips \& Pflieger (2000) hypothesized that evoked EEG responses, which are phase-locked to the stimulus, reflect RF processing; whereas induced EEG responses, which are event-related but not phase-locked to the stimulus, reflect CF processing. Grounds for thinking that this may approximately hold, are: (a) phase-locking to the stimulus naturally suggests external eventdriven processing; and (b) non-phase-locking with respect to the stimulus may reflect ongoing cortical coordinating activity with intrinsic timing. We devised a high-density EEG pilot study (unpublished) that used complex visual contour integration stimuli consisting of many small Gabor patches (Field et al. 1993), as specially modified by George Lovell (see his submitted doctoral thesis [Lovell 2002]). Pairs of stimuli were presented simultaneously to the right and left visual fields. One stimulus consisted of structured sets of Gabor patches with perceived contours embedded in a background of randomly oriented patches. The second stimulus consisted of the same configuration with 40-degree random jittering of patch orientations (which obliterated perceived contours). The subject's task was to detect "structure right" or "structure left." Average reaction times were about 400 msec with nearly $100 \%$ accuracy. Between-condition differences for the broadband average event-related potentials (about 275 epochs per condition) were subtle at best for all scalp locations up to about $250 \mathrm{msec}$. Evoked and induced responses were calculated using the method of complex demodulation for the following symmetric bands: 10 $\mathrm{Hz} \pm 5 \mathrm{~Hz}, 20 \mathrm{~Hz} \pm 10 \mathrm{~Hz}, 30 \mathrm{~Hz} \pm 15 \mathrm{~Hz}$, and $40 \mathrm{~Hz} \pm 20 \mathrm{~Hz}$. A nonparametric permutation procedure was used to convert all differences to uncorrected $p$-value waveforms, with low values (close to 0 ) indicating left > right visual field differences, and high values (close to 1 ) indicating right $>$ left visual field differences. Significant induced and evoked $40 \mathrm{~Hz}$ differences were observed: Right $>$ left at left para-occipital locations at about $32 \mathrm{msec}$ (induced) and 34 msec (evoked), and left $>$ right at right para-occipital locations at about $76 \mathrm{msec}$ (induced) and $58 \mathrm{msec}$ (evoked). Similar induced, but not evoked, $30 \mathrm{~Hz}$ response differences were observed. No induced $20 \mathrm{~Hz}$ or $10 \mathrm{~Hz}$ differences attained significance, nor did the evoked $20 \mathrm{~Hz}$ differences. Evoked $10 \mathrm{~Hz}$ differences were about $36 \mathrm{msec}$ for right $>$ left (left para-occipital) and about 26 msec for left $>$ right (right para-occipital). Therefore, both induced and evoked differences were found at the expected sides, with generally slower latencies for structure on the left.

By definition, these subtle differences reflect contextual field processing. Because evoked differences sometimes precede induced differences, this single subject pilot study does not support our hypothesis. However, the finding of relatively early $40 \mathrm{~Hz}$ band differences may be meaningful in the context of $P \& S$.

\section{ACKNOWLEDGMENTS}

The visual contour integration experiment was designed in collaboration with Bill Phillips. EEG data were acquired in collaboration with Steve Sands at Neuroscan. Preliminary data sets were acquired in the lab of Tom Nakada at Niigata University in collaboration with Shugo Suwazono.

\section{NMDA synapses can bias competition between object representations and mediate attentional selection}

\author{
Antonino Raffone ${ }^{a}$, Jaap M. J. Murre ${ }^{b}$, and \\ Gezinus Wolters ${ }^{c}$ \\ a Department of Psychology, University of Sunderland, St. Peter's Campus, \\ SR6 ODD Sunderland, United Kingdom; ${ }^{\mathrm{b}}$ Department of Psychology, \\ University of Amsterdam, 1018 WB Amsterdam, and University of Maastricht, \\ The Netherlands; ' ${ }^{\circ}$ Department of Psychology, Leiden University, 2300 RB \\ Leiden, The Netherlands. antonino.raffone@sunderland.ac.uk \\ jaap@murre.com wolters@fsw.leidenuniv.nl
}

Abstract: Phillips \& Silverstein emphasize the gain-control properties of NMDA synapses in cognitive coordination. We endorse their view and suggest that NMDA synapses play a crucial role in biased attentional competition and (visual) working memory. Our simulations show that NMDA synapses can control the storage rate of visual objects. We discuss specific predictions of our model about cognitive effects of NMDA-antagonists and schizophrenia

Phillips \& Silverstein's (P\&S’s) target article is a major step in the development of a coherent theoretical framework for understanding how spatially distributed brain activity is coordinated or integrated in normal function, and how this coordination may be impaired in schizophrenia. Their perspective reconciles localist and holist approaches to brain function into a dialectic functional scheme, in which invariant local (modular) processing operations or neural representations are flexibly integrated into larger context- or task-dependent processing or representational units.

We endorse P\&S's view on the crucial role of NMDA synapses in cognitive coordination and control, based on their amplifying voltage-dependent properties. Specifically, the gain-control properties of NMDA synapses on ongoing processing discussed in the target article may be plausibly involved in selective encoding and maintenance of task-relevant information in cortical networks of (visual) working memory.

In our neurocomputational approach to maintenance and control in visual working memory (Raffone \& Wolters 2001; Raffone et al. 2001), the neural representations of features and objects are kept active after stimulus-offset by feedback connections from prefrontal cortex to posterior cortical areas. In this model, object features are integrated by synchronizing connections (within-chunk integration), and different objects are segregated by fast mutual inhibition (between-pattern segregation). The storage capacity reflects the equilibrium point between these two neural representational forces. In a current study (Raffone et al., in preparation), cortical feedback, within-chunk integration, and between-pattern segregation are combined with NMDA synapses. In this commentary we will outline how NMDA synapses can play a crucial role in biased attentional competition (Desimone \& Duncan 1995) between neural representations of objects, and discuss specific implications and predictions from our model consistent with P\&S’s general perspective.

Competition between object representations is a fundamental aspect in many theories of visual attention. For instance, in Bundesen's (1990) Theory of Visual Attention (TVA), the units of visual representation are parallel categorizations of visual elements in limited-capacity visual short-term memory. These categorizations compete in terms of parallel processing speed (affecting the storage probability), which depends on the relative attentional weight of visual elements. Theories of attention often refer to the concept of biased competition principle between object representations (Desimone \& Duncan 1995). According to the biased competition model, the attentional weights (saliency or priority values) of different objects (saliency or priority values) are correlated with selectively biased firing rates of associated competing neural representations in the visual cortex.

It is generally assumed that attentional weights are encoded in terms of firing rate modulation by top-down cortical feedback, plausibly from prefrontal cortex. NMDA synapses, because of their voltage-dependent properties, can implement a gain mechanism in which bottom-up signals are selectively amplified, de- 
Commentary/Phillips \& Silverstein: Convergence of perspectives on cognitive coordination in schizophrenia

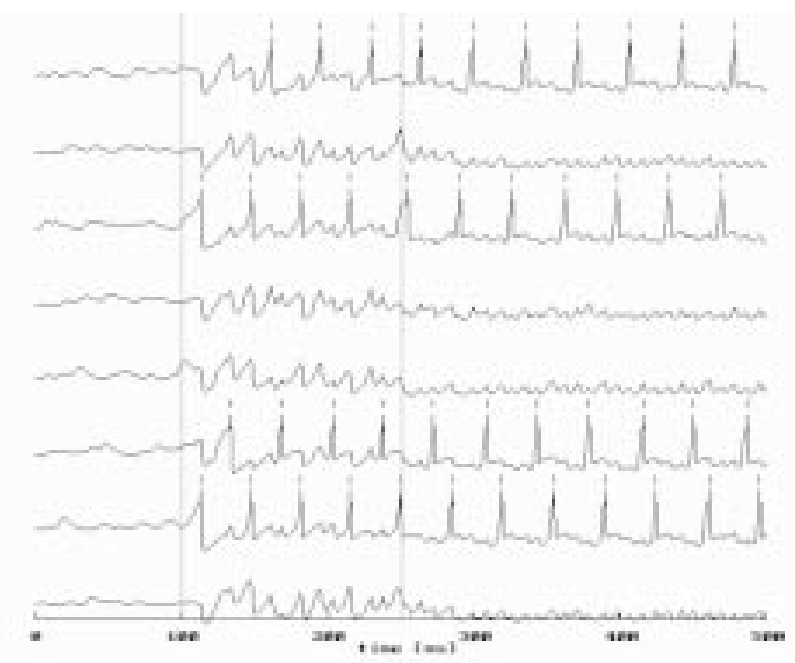

A

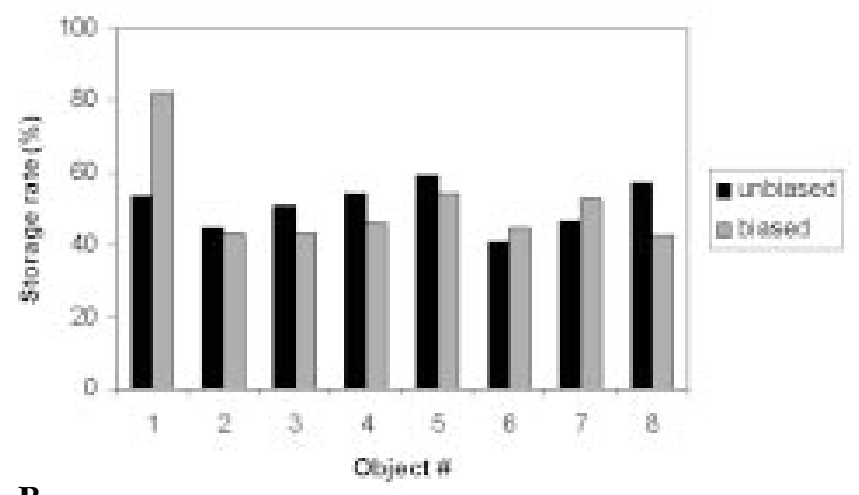

B

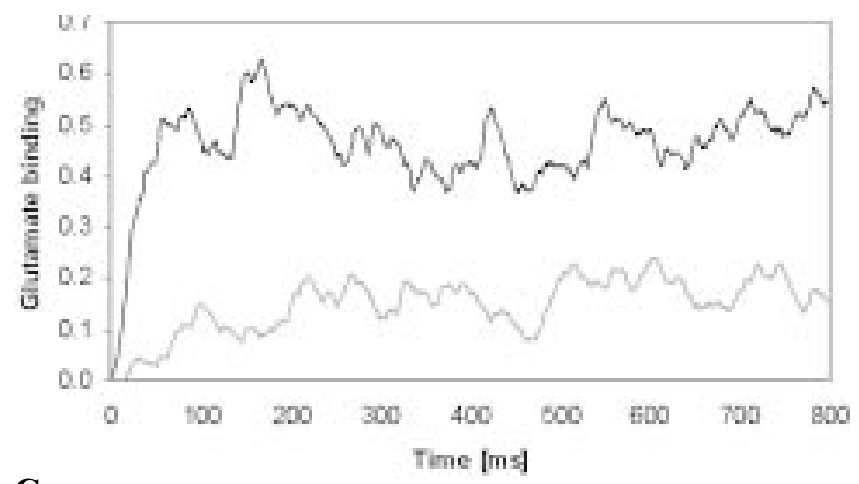

C

Figure 1 (Raffone et al.). NMDA-dependent biased competition effects in visual working memory storage. (A) Typical evolution of membrane potentials of eight model neurons from eight parietal assemblies coding for eight different objects (stimulus onset is at $100 \mathrm{msec}$, and offset at $250 \mathrm{msec}$; only four objects are maintained after stimulus offset). (B) Percent storage rate of eight objects with (grey bars) and without (black bars) bias toward Object 1 , over 200 trials with a storage capacity of about four objects. (C) Glutamate binding at NMDA synapses for attended (black curve) and unattended (grey curve) objects. See text for further explanations.

pending on their top-down saliency. They can, therefore, implement a multiplicative computational scheme in attentional processing, as in Bundesen's (1990) TVA.

We have simulated this NMDA-dependent gain effect in visual working memory storage (Raffone et al., in preparation). In the model, separate objects are encoded by disjoint sets of recur- rently-coupled neural assemblies located in the inferotemporal cortex, parietal cortex, and prefrontal cortex. Neural assemblies in parietal cortex receive top-down NMDA signals from control assemblies with a differential firing rate located in a prefrontal area.

In the simulation series shown in Figure 1, the cortical network model was presented with displays of eight objects. Given that on average only four objects are stored in terms of reverberations in the absence of any attentional bias (Raffone \& Wolters 2001), the storage rate for each object is about $50 \%$. Figure 1A shows the typical evolution of membrane potentials (firing patterns) of eight parietal neurons coding for different objects, with interneuron time-lags reflecting between-pattern segregation. In Figure 1B, the storage rate of eight objects over 200 trials is shown with and without attentional bias toward Object 1 . Note the higher storage rate of Object 1 with the amplifying effect of NMDA function (in terms of latent glutamate binding, see Fig. 3 in the target article) added to the parietal assembly coding for it, as compared with the unbiased condition. All the other structural and dynamic parameters are kept constant across the two conditions. Figure 1C shows the evolution of latent NMDA synaptic signals (amount of bound glutamate modeled in terms of a dual exponential with rise time of $5 \mathrm{msec}$ and decay time of $50 \mathrm{msec}$ ) to target and distractor assemblies in the biased attentional weight condition.

As shown by our simulations, an important property of NMDA signals is that they are voltage-dependent, and therefore are enabled only in the presence of bottom-up input (sensory evidence). In our model, this dynamic property is also crucial to prevent spurious activation spreading to irrelevant neurons by feedback connections from prefrontal cortex during maintenance. In this case, NMDA signals are generated at the level of recurrent connectivity intrinsic to neural assemblies in posterior areas, and top-down signals from prefrontal cortex selectively act on non-NMDA input-activated assemblies with a sufficient amount of intrinsic glutamate binding. Therefore, reverberations for maintenance only capture input-activated neurons in feature and location dimensions. The absence of NMDA from this circuitry would imply either reduced maintenance capacity or the emergence of hallucinatory neural representations.

If NMDA synaptic systems are impaired in schizophrenia, as suggested by $\mathrm{P} \& \mathrm{~S}$ in the target article, and if NMDA synapses play a crucial role in attentional selection and control of storage and maintenance in visual working memory, as suggested by our model, then differences between groups of schizophrenic patients and control groups are predicted in cognitive tasks with interactions between visual working memory and selective attention. Similar functional and pathological principles may be involved in auditory (verbal) working memory and selective attention. Our model also predicts hallucinations as a result of spurious activation spreading from prefrontal cortex to posterior cortical areas under the effect of NMDA-antagonists, and, following P\&S, in schizophrenia.

\section{Why do schizophrenic patients hallucinate?}

\section{Pieter R. Roelfsema ${ }^{a}$ and Hans Supèra, b \\ a The Netherlands Ophthalmic Research Institute, 1105 BA Amsterdam, The Netherlands; 'b Graduate School of Neurosciences Amsterdam, Department of Visual System Analysis, Academic Medical Center (UvA), 1100 AA Amsterdam, The Netherlands. \{p.roelfsema;h.super\}@ioi.knaw.nl}

Abstract: Phillips \& Silverstein argue that schizophrenia is a result of a deficit of the contextual coordination of neuronal responses. The authors propose that NMDA-receptors control these modulatory effects. However, hallucinations, which are among the principle symptoms of schizophrenia, imply a flaw in the interactions between neurons that is more fundamental than just a general weakness of contextual modulation.

The target article distinguishes between "primary input" and "contextual input" onto a neuron. Primary input drives the cell and 
is generally attributed to feedforward connections that propagate activity from lower to higher areas (Felleman \& Van Essen 1991). Contextual input, on the other hand, does not activate a neuron, but rather modulates its response. One source of contextual effects in vision is the stimulus context, because properties of the stimulus outside the receptive field can modify neuronal responses. These contextual effects are usually attributed to preattentive vision (Lamme \& Roelfsema 2000). Task requirements are another source of contextual effects that depend on shifts of visual attention (Roelfsema et al. 2000).

Both types of contextual influences are particularly strong for neurons that are well driven by the visual stimulus, and much weaker for cells that receive little bottom up activation (Kapadia et al. 1995; Knierim \& Van Essen 1992; McAdams \& Maunsell 1999; Zipser et al. 1996). We agree with the target article that contextual effects appear to multiply the effect of driving connections, by some factor. Here we focus on the multiplicativity of this interaction, and argue that it is important for the specificity of both preattentive and attentive response modulation.

Visual search is an example of an attention-demanding task that depends on interactions between cortical areas, and that is impaired in schizophrenia (Lubow et al. 2000). In visual search, the identity of an item is given and its location has to be determined. This implies an interaction between ventral stream areas encoding the identity of the target item, and areas in the dorsal stream, as well as early visual areas that encode locations. A scheme that incorporates the essential ingredients of contemporary models of search is indicated in Figure 1. Three areas have been indicated, at different levels of the visual cortical hierarchy. In this simplified scheme, two features (a and b) are represented in each of the areas. Feedback connections interconnect neurons with a similar feature preference. Neurons that are driven by feedforward connections are indicated as black circles. Search for a particular target feature (e.g., feature a) can be implemented by feedback from higher areas to enhance the activity neurons in lower areas that have the target feature in their receptive field (Roelfsema et al. 2000; van der Velde \& de Kamps 2001).

Figures 1B and 1C compare the effects of multiplicative (modulatory) and additive (driving) feedback. If feedback is multiplicative, as in Figure 1B, only neurons that receive bottom-up support from feedforward connections are influenced by it, and feedback does not spill over to neurons without a matching feature in their receptive field (stippled connections in Fig. 1B are disabled since there is no bottom-up input to these cells). When the search has been completed, the location of the target item is encoded by a response enhancement in early visual areas, which can be read out by areas of the dorsal stream (van der Velde \& de Kamps 2001). Figure 1C shows that this focusing of activity at the location of the target item cannot be achieved by additive feedback (driving connections), because it would also activate neurons at locations of distractors (e.g., connection 1 in Fig. 1C). Moreover, additive feedback can even activate neurons at locations where there are no objects at all (connection 2). Grossberg argued that feedback therefore has to be multiplicative, to prevent hallucinations (Grossberg 1999). Models of "preattentive" texture segregation have also exploited the virtues of multiplicative feedback, to enhance activity in early visual areas evoked by a figure over activity evoked by the background (Roelfsema et al. 2002).

The proposal of the target article that contextual effects are mediated by NMDA-receptors would account for the multiplicative interaction between contextual and driving connections (Fox et al. 1990). Current can flow only through NMDA-channels of neurons that are sufficiently depolarized by driving connections to remove the Mg-block. We note, however, that certain anesthetic agents not acting on NMDA-receptors but operating through different routes, also interfere with contextual modulation without an effect on driving connections (Lamme et al. 1998; Pack et al. 2001). Nevertheless, we agree that the possibility that NMDA-receptors are important for contextual modulation is exciting and worth testing.

But what about hallucinations? They are among the defining
A Model
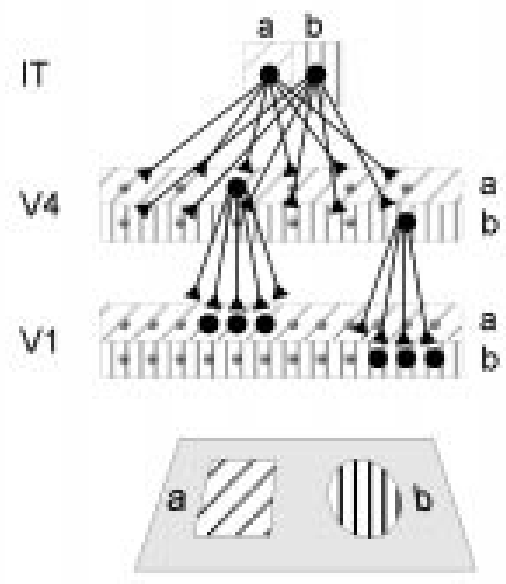

\section{B Multiplicative feedback}

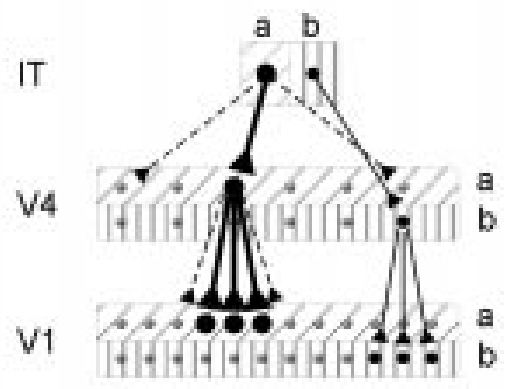

\section{Additive feedback}

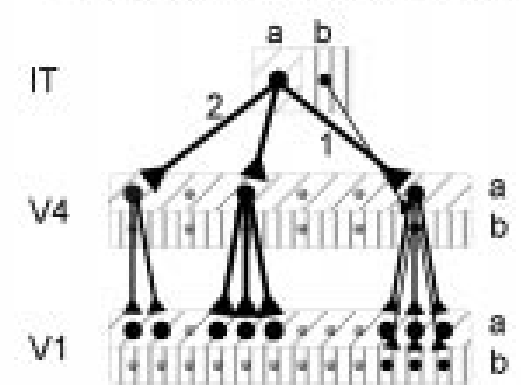

Figure 1 (Roelfsema \& Supèr). Involvement of feedback connections in visual search. (A) In models of visual search, feedback connections are assumed to interconnect neurons with a similar feature preference. Two features $(a$ and $b)$ are represented in three areas of the temporal stream of the visual cortex. In reality, more stages are interposed between the primary visual cortex (area V1) and the inferotemporal cortex (area IT). Neurons that are activated by the stimulus below (by feedforward, driving connections, not shown) have been indicated as black circles. Neurons not activated by the visual stimulus are indicated as small gray circles. (B) Multiplicative feedback. Connections to neurons not activated by the input have no effect (stippled connections). Visual search for feature $a$ can be implemented by feedback from area IT, enhancing neuronal responses to the target feature " $a$ " (thick connections) in lower visual areas. In retinotopic areas, the response enhancement occurs at the target object's location, and this solves the search task. (C) Additive feedback activates neurons at lower hierarchical levels that are not activated by bottom up. It activates neurons at the location of the distractor object (connection 1), and even at locations without an object (connection 2), causing hallucinations. 
symptoms of schizophrenia. We have just argued that the multiplicative interaction between driving and contextual connections is important to avoid hallucinations, since hallucinations may occur if feedback drives neurons in early sensory areas that are not activated by bottom-up. This multiplicativity may be lost in schizophrenia; recent imaging work demonstrates that the primary auditory cortex is activated by auditory hallucinations in schizophrenic patients (Dierks et al. 1999). This implies that feedback in schizophrenia is too strong, because it can create activity patterns in sensory areas that are not supported by the sensory organ itself - and contradicts Phillips \& Silverstein's (P\&S's) hypothesis that the main deficit in schizophrenia is a weakness of contextual modulation.

We conclude that a general weakness of contextual effects cannot be the only deficit in schizophrenia. Mechanisms other than contextual modulation must be disturbed in the communication between neurons in the same and different brain areas. Unfortunately, we still know too little about these interactions, even in normal subjects. Some hold, for example, that they are carried by synchronous neuronal rhythms (Phillips \& Singer 1997a; Singer \& Gray 1995), whereas others argue that they are rather associated with the modulation of firing rates (Desimone \& Duncan 1995; Lamme \& Roelfsema 2000), although these possibilities are not mutually exclusive. The target article is an important contribution because it pinpoints our lack of knowledge about how neurons in different cortical areas exchange information. Moreover, it provides an inspiration to study disturbances in the neuronal interactions to gain insight in the pathophysiology of schizophrenia.

\section{No blind schizophrenics: Are NMDA-receptor dynamics involved?}

\author{
Glenn S. Sanders ${ }^{\mathrm{a}}$, Steven M. Platek ${ }^{\mathrm{b}}$, and \\ Gordon G. Gallup, Jr. ${ }^{a}$
}

a Department of Psychology, State University of New York at Albany, Albany, NY 12222; ' Department of Psychology, Drexel University, Philadelphia, PA 19102. steven.m.platek@drexel.edu

http://www.pages.drexel.edu/ smp43 gallup@csc.albany.edu http://www.albany.edu/ gallup http://www.evolutionarypsych.com

\begin{abstract}
Numerous searches have failed to identify a single co-occurrence of total blindness and schizophrenia. Evidence that blindness causes loss of certain NMDA-receptor functions is balanced by reports of compensatory gains. Connections between visual and anterior cingulate NMDA-receptor systems may help to explain how blindness could protect against schizophrenia.
\end{abstract}

Phillips \& Silverstein (P\&S) have pieced together a plausible and detailed connection between NMDA-receptors and schizophrenia. Our commentary is intended to illustrate the idea that receptor hypofunction is a dynamic process that in certain cases may lead to compensatory and protective neural circuitry. We first consider implications of research on early sensory deprivation, and then provide substantiation for our claim (Sanders et al. 2002) that blind people do not become schizophrenic.

Variation in sensory experience has important effects on cerebral architecture and connectivity (overview in Geary \& Huffman 2002). Some of this impact applies directly to blindness and NMDA-receptor structure and function. For example, when marmosets undergo monocular eyelid suture, their ocular dominance columns exhibit suppressed NMDA-receptor NR1 subunit immunoreactivity (Fonta et al. 2000). This suggestion of impaired receptor integrity is consistent with reductions in NMDA-evoked, but not baseline, calcium uptake in the striate cortex of darkreared kittens (Feldman et al. 1990). Dark-rearing also increases sensitivity of rats' visual cortex NMDA-receptors to certain chemical antagonists (Fathollahi \& Salami 2001), which may be a reflection of receptor loss or abnormality.
The impact of visual deprivation on NMDA-receptors probably extends beyond the occipital cortex. Given the substantial role of the anterior cingulate cortex (ACC) in cognitive coordination and in schizophrenia (Sanders et al. 2002), it is noteworthy that ACC and primary visual cortex have strong physical (Vogt \& Miller 1983) and functional (Nishijo et al. 1997) connectivity mediated by glutamate transmission and NMDA-receptors (Sah \& Nicoll 1991). As a result, hypofunction in visual cortex may lead to diminished input for and altered structure or function of NMDAreceptors in the ACC. Consistent with this possibility, the ACC participates in the generation of visual images (Deiber et al. 1995), and the visual images of blind people suffer from deficiencies in dynamic grouping and context sensitivity (DeBeni \& Cornoldi 1988), which the target article attributes to reduced ion flow in NMDA-receptors.

Despite indications of widespread NMDA abnormality in blind people, there is no evidence that they develop the severe cognitive coordination problems characteristic of some types of schizophrenia. On the contrary, blind people seem to be completely protected. Five independent searches, varying considerably in scope, methods, and population, failed to identify even one well-defined co-occurrence of total blindness and schizophrenia (Abely \& Carton 1967; Chevigny \& Braverman 1950; Feierman 1982; Horrobin 1979; Riscalla 1980). We dedicated portions of 2000 and 2001 to e-mail and postal mail surveys of relevant professionals; e-mail and telephone discussions with officials of health, mental health, blindness, and schizophrenia organizations and research institutes; and extensive keyword probes of Medline, PsychINFO, and ScienceDirect databases. Some ambiguity was introduced by very low return rates for our surveys, but the consistent result of all these inquiries was that no instance of totally blind/schizophrenic co-occurrence was found.

How could blindness protect against schizophrenia? Our hypothesis (Sanders et al. 2002) centers on the idea that certain compensations in the ACC for loss of visual input may provide "immunity." Blindness leads to neural reorganization and restructuring (Hamilton \& Pascual-Leone 1998; Liotti et al. 1998). Psychologically, these cortical changes allow the blind to achieve normal cognitive development (Warren 1994) despite formidable challenges (e.g., Sandler \& Hobson 2001). One example of the compensatory reactions to blindness is the development of aboveaverage verbal memory (Decker \& Koole 1992).

We believe that some of these blindness-induced neural and psychological compensations involve the dynamics of NMDAreceptor function. Evidence of deprivation-induced receptor hypofunction is balanced by a report of upregulation of NMDAreceptor $1 \mathrm{~A}$ subunits in optic and intermediate gray layers of superior colliculus resulting from peripheral blindness (Vizuete et al. 2001). This upregulation led to an increase in corticocollicular synapses as a compensation for the loss of retinocollicular interaction. In the same vein, dark-rearing forestalls the normal upregulation of NMDA-receptor 2A subunits in striate cortex (Quinlan et al. 1999). This interference with normal receptor elaboration has the paradoxical, or compensatory, effect of enhancing NMDA-receptor activity, ultimately prolonging the critical period for plasticity and modulation in the visual cortex (Fox et al. 1999). Such trade-offs between loss and gain in visual system NMDA-receptors of blind people may help explain the mature viability and reorganization of a sensory region deprived of its primary afferents (Breitenseher et al. 1998).

As was the case for losses discussed above, there is reason to believe that blindness-induced NMDA-receptor gains in primary visual system are matched in other regions, particularly the ACC. A pertinent example is the report of a larger mismatch negativity (MMN) evoked-potential response to deviant auditory stimuli in blind than in sighted humans (Kujala et al. 1995). This demonstration of enhanced context sensitivity in the blind stands in stark contrast to the diminished MMN (mismatch negativity) observed in schizophrenics, and also in control samples administered the NMDA-receptor antagonist ketamine (Umbricht et al. 2000) 
Strong involvement of ketamine in ACC function is firmly established, and the ACC is a relatively late (top down?) generator of MMN (Waberski et al. 2001). It is reasonable to speculate that the dynamics of blindness-induced NMDA-receptor hypofunction in visual cortex extends to NMDA-receptor gains in the ACC, which in turn increase some types of context sensitivity, perhaps ultimately resulting in protection against schizophrenia.

In the light of these results, the answer to point 11 in P\&S's concluding section - "Is the molecular and regional diversity of NMDA receptor channels ... crucial?" (target article, sect. 8) - is likely to be "Yes!" (see de Belleroche et al. 1998 for direct evidence). The impression of dynamic, heterogeneous, and compensatory processes produced by research on visual deprivation is enhanced when deafness is added to the picture. Loss of auditory input produces a pattern of loss and gain in NMDA-receptor function in some ways similar to and in other ways different from the effects of dark-rearing or blindness (e.g., Nakagawa et al. 2000; Oleskevich \& Walmsley 2002). Most unlike the effects of blindness, deafness does not serve to prevent schizophrenia (e.g., Schonauer et al. 1999).

Many of the issues raised by P\&S may be better understood following certain comparisons among blind, deaf, schizophrenic, and control samples. For example, how might blind people respond to ketamine antagonism of their ACC NMDA-receptors? It would be especially interesting to extend to blind and deaf samples the work on cortical rhythms reviewed in section 5 of the target article. Patterns of beta and gamma frequencies, and their modulation by ketamine, may reveal differences in local and top-down cognitive coordination in blind versus deaf people, thus helping explain the dramatic difference in their susceptibility to schizophrenia.

\section{Phenomenology, context, and self-experience in schizophrenia}

\author{
Louis A. Sass ${ }^{a}$ and Peter J. Uhlhaas ${ }^{b}$ \\ araduate School of Applied and Professional Psychology, Rutgers \\ University, Piscataway, NJ 08854-8085; ' Center for Computational and \\ Cognitive Neuroscience, Department of Psychology, University of Stirling, \\ Stirling, FK9 4LA England. Isass@rci.rutgers.edu \\ uhlhaas@hotmail.com http://gsappweb.rutgers.edu/gsappweb/sass
}

\begin{abstract}
Impairments in cognitive coordination in schizophrenia are supported by phenomenological data that suggest deficits in the processing of visual context. Although the target article is sympathetic to such a phenomenological perspective, we argue that the relevance of phenomenological data for a wider understanding of consciousness in schizophrenia is not sufficiently addressed by the authors.
\end{abstract}

Phillips \& Silverstein $(\mathrm{P} \& S)$ propose an innovative model of the characteristic cognitive disorganization of schizophrenia, based on neurobiological, pharmacological, and cognitive data. The authors also refer to the classic psychiatric writings of Matussek (1952/ 1987) and Conrad (1958), who adopt a Gestaltist perspective and describe subjective experience in schizophrenia in detail. Although we applaud the mention of such Gestaltist work with its emphasis on phenomenological data, we believe that the theory of P\&S neglects important aspects of consciousness in schizophrenia that are central to the understanding of the condition.

In Anglophone psychiatry, phenomenology generally refers to the study of observable signs and symptoms. In philosophy and phenomenological psychiatry, however, phenomenology refers to the systematic study of conscious experience from a first-person perspective and is tied to certain philosophical positions regarding mind and its relationship to the world. We believe that inclusion of a phenomenological perspective (in the second sense) is essential to gain insights into the cognitive abnormalities and their wider relevance for understanding the schizophrenic condition (Sass \& Parnas, in press a).
Gurwitsch (1964) offers a phenomenological analysis that may help to clarify the subjective dimension of these disorders and the role of context in consciousness. In Gurwitsch's view, the field of consciousness always involves a "theme," "thematic field," and "margin" of awareness. Gurwitsch's first domain, the "theme," is that which engrosses the subject, stands in the focus of his attention, and "upon which his mental activity concentrates" (p. 4). The theme is characterized by the kinds of relationships classically described by Gestalt psychology: Here, each constituent "exists in the very qualifications by which it is defined and made to be that which it is in a given case, only in conjunction with, and determined by, co-constituents" (p. 139). The second domain, the "margin," is the realm of objects of awareness that are simultaneously present to consciousness but not experienced as relevant to the focal theme; it includes elements of ongoing bodily existence, the perceptual surround, and the stream of consciousness (p. 11). The third domain - a kind of middle realm - is that of the "thematic field," defined as "the totality of facts, co-present with the theme, which are experienced as having material relevancy or pertinence to the theme" (p. 55).

Constituents of the thematic field are not merely copresent; they hang together in a mutually implicative way, and have relevance for the focal theme. Thus, the way in which each of these constituents is experienced is a function of some larger unifying significance that also determines the aspect of the theme that will emerge as significant. Gurwitsch (1964) speaks here of "unity by relevancy," which is bound up (as both source and product) with the unity of context (p. 341); it can be distinguished from the even tighter form of unity inherent in "Gestalt-coherence."

The main disturbance in schizophrenia, according to $\mathrm{P} \& \mathrm{~S}$, concerns the capacity for "context-processing." From a phenomenological and experimental perspective, a disturbance in contextprocessing is most obvious at the level of context that Gurwitsch calls the "the thematic field," because schizophrenia patients are not deficient in the ability to perceive basic Gestalts (Knight et al. 2000; Sass, in press). The work of Matussek (1952/1987) and Con$\mathrm{rad}$ (1958) supports this view. Examination of Matussek's clinical illustrations shows that there is seldom, if ever, a breakdown of the basic spatial form; indeed, the integrity of the visual object may even be heightened as it stands out with special clarity while the background recedes in dullness and indifference (Matussek 1952/ 1987, p. 92). The main perceptual alteration Matussek emphasizes is a "loosening of the natural perceptual context" or of the "coherence of perception."

Impairment in context-processing at the level of the thematic field has wide implication for the understanding of consciousness in schizophrenia. According to Gurwitsch (1964), the "thematic field" determines the "perspective under which, the light and orientation in which, the point of view from which" the theme appears to consciousness (p. 359). Accordingly, weakening of the "thematic field" is associated with a loosening of perceptual context that may alter the organization or salience of the theme. The phenomenological evidence suggests that this is so. Matussek, for example, describes everyday objects that are framed in isolation, and thus come to seem strange or to acquire exaggerated and often enigmatic symbolic "weighting" as a result of the "loosening of the natural perceptual context” (Matussek 1952/1987).

Relevancy relationships (characteristic of the thematic field) are the kind that are most directly related to the ongoing projects or concerns of the subject; for this reason they may be especially closely linked to fundamental aspects of self-experience, that is, to the underlying sense of existing as a vital and unified subject of awareness or first-person perspective, which has been referred to as "ipseity" (from the Latin ipse, which means self or itself; Sass 2000; Sass \& Parnas, in press b). The typically schizophrenic alterations of the cognitive/perceptual field are, in fact, usually accompanied by disruptions in this most basic sense of selfhood (Klosterkoetter et al. 1997; Møller \& Husby 2000; Parnas et al. 1998). Such a patient may complain of an "inner void," of "painful distance to self," of being "occupied by, and scrutinizing, my own 
inner world," of not feeling himself, or even having no consciousness at all (Møller \& Husby 2000, pp. 221-23, 228).

Normal ipseity (and the normal tacit-explicit structuring of experience this implies) is a condition for the experience of appetite, vital energy, and point of orientation: It is what grounds human motivation and organizes our experiential world in accordance with needs and wishes, thereby giving objects their affordances - their significance for us as obstacles, tools, objects of desire, and the like. In the absence of this vital self-affection and the lines of orientation it establishes, there can no longer be clear differentiation of means from goal; no reason for certain objects to show up in the focus of awareness while others recede; no reason for attention to be directed outward rather than inward toward one's own body or processes of thinking (i.e., "hyperreflexivity"; Sass 2000).

Most attempts to explain the disturbances of schizophrenic cognition have assumed they are rooted in purely cognitive and often rather modular dysfunctions, such as associational disturbance, failures of attention or working memory, or an incapacity for the planning or monitoring of discourse or thought. Such functions may indeed be abnormal. It is possible, however, that these disturbances are secondary to a more fundamental distortion of psychological processes that are relevant for adopting a practical, goal-oriented stance toward the world, and for constituting a lived point of orientation and the correlated pattern of meanings that make for a coherent and significant world. We believe that P\&S's view of schizophrenia as an "impairment in cognitive coordination" may be relevant for identification of such a fundamental distortion of psychological processes in schizophrenia. However, future research would benefit from incorporating phenomenological approaches to examine relationships between disorders of cognition and self-experience.

\section{Cortical connectivity in high-frequency beta-rhythm in schizophrenics with positive and negative symptoms}

\author{
Valeria Strelets \\ Institute of Higher Nervous Activity and Neurophysiology, Russian Academy \\ of Sciences, 117485 Moscow, Russia. strelets@aha.ru
}

Abstract: In chronic schizophrenic patients with both positive and negative symptoms (see Table 1), interhemispheric connections at the high frequency beta2-rhythm are absent during cognitive tasks, in contrast to normal controls, who have many interhemispheric connections at this frequency in the same situation. Connectivity is a fundamental brain feature, evidently greatly promoted by the NMDA system. It is a more reliable measure of brain function than the spectral power of this rhythm.

Recently we have studied several EEG measures revealing some abnormalities in schizophrenia (Burgess et al., submitted; Strelets et al., under review). In our work aimed at the study of different brain rhythms' spectral power and connectivity, it was revealed that, in schizophrenic patients with positive and negative symptoms, the spectral power of all brain rhythms except the beta2rhythm was decreased in comparison with normal controls (Strelets et al. 2002). We refer to this rhythm, consisting of oscillations with a frequency of $20-40 \mathrm{~Hz}$, more precisely as $38 \mathrm{~Hz}$. Therefore, the spectral power of this rhythm in schizophrenic patients does not differ significantly from that of the normal controls, though the literature on this point is controversial. The interhemispheric connections at this rhythm, studied by the coherence method, in contrast to those in normal controls, were absent, while at other rhythms we can observe in patients normal connections or even hyperconnectivity. One can propose that the high frequency rhythms aren't well organized enough to form necessary connections during cognitive tasks in schizophrenic patients. The glutamatergic ionotropic NMDA system and other neurotransmitters evidently play a great role in this disorganization of connectivity at the beta2rhythm in schizophrenic patients.

The EEG was recorded from ten derivations using the standard scheme in the rest condition (eyes closed) and during task performance - silently counting the hours on an imaginary clock dial.

EEG traces of 100 seconds were recorded. Subsequent analysis of each EEG segment consisted of selection of 10 to 20 fivesecond EEG fragments, free from artifacts. These fragments underwent Fast Fourier Transform, the results of this procedure being used for subsequent analysis.

The study of connectivity was carried out using two methods: (1) Coherence, the most commonly used method - measure of synchronization, based on the evaluation of functional integration between the brain areas at the frequency, averaged across frequency domain for each subject; and (2) the method of typical connections analysis, developed by our group (Strelets et al. 2002), based on the analysis of the peaks, precisely coinciding in frequency in individual power spectra of different derivations for each frequency domain. The most typical connections for the group were selected by their probabilities, and their significance was tested by a Monte-Carlo method. Therefore, this method enables us to detect the real (not averaged) frequency at which the connections between cortical areas are established.

Coherence study. In the rest condition in the beta2-range (20$40 \mathrm{~Hz}$ ), in normal subjects there were two interhemispheric connections. During the task performance, the number of connections at this rhythm increased to eight in comparison with the rest condition. The connections were revealed mostly in the anterior cortical areas.

In patients with positive symptoms, in the beta2-range interhemspheric connections were absent, and the number of intrahemispheric connections was significantly more, the latter including the temporal areas. During task performance, a paradoxical reaction was observed in this group: opposite to the norm, the number of connections decreased compared to the rest condition.

In comparison with the norm, in both experimental situations patients with negative symptoms had no interhemispheric con-

Table 1 (Strelets). Demographic characteristics of three subject groups

\begin{tabular}{lccc}
\hline \hline & & Group & \\
\cline { 2 - 4 } & Normals & Patients with “positive" symptoms & Patients with "negative” symptoms \\
\hline Age (years) & $20-50(32.56 \pm 8.67)$ & $25-47(33.21 \pm 7.94)$ & $20-53(34.94 \pm 9.18)$ \\
Education (years) & $10-15(12.56 \pm 2.25)$ & $8-15(11.25 \pm 2.40)$ & $7-15(10.20 \pm 62.52)$ \\
The age of first onset (years) & - & $17-39(26.57 \pm 5.80)$ & $19-53(28.10 \pm 10.95)$ \\
Chronicity: & - & $0-9(1.42 \pm 1.31)$ & $0-4(1.05 \pm 0.93)$ \\
$\quad \begin{array}{l}\text { since the last admission (years) } \\
\text { since the first admission (years) }\end{array}$ & - & $0-23(6.43 \pm 6.01)$ & $0-29(6.68 \pm 6.16)$ \\
Number of admissions & - & $1-24(5.34 \pm 5.11)$ & $1-9(3.47 \pm 2.34)$ \\
\hline \hline
\end{tabular}


nections in the beta2-range, revealing pathological rigidity. During task performance, the differences in coherence patterns between these two groups were insignificant.

As we see from the above, the results obtained in the coherence study showed dramatic results: the absence of interhemispheric connections in both patient groups in comparison with the norm at the frequency $20-40 \mathrm{~Hz}$ (beta2) in all conditions. At the same time there were no significant differences between the two patient groups.

Typical connections study. In the normal group in rest condition, there were two interhemispheric and three intrahemispheric connections at the frequency $38 \mathrm{~Hz}$. During the task, the number of connections at this frequency increased to four interhemispheric and five intrahemispheric ones, with the predominant inclusion of areas of the left hemisphere. In patients with positive symptoms in the rest condition, in the beta2-range interhemispheric connections were absent. In comparison with the norm, in this group there were significantly more connections of temporal areas with other ones. During task performance in patients with positive symptoms, typical interhemispheric connections in the beta2-range were also absent.

In patients with negative symptoms, in the beta2-range (20-40 $\mathrm{Hz}$ ) typical connections were revealed only in the left hemisphere. The interhemispheric connections were absent in both situations.

The comparison between the patients with positive and negative symptoms during task performance revealed in the beta2range $(20-40 \mathrm{~Hz})$ a sort of "parity" in the number of significant differences between these groups, but patients with negative symptoms had more connections in medial areas, including interhemispheric ones, and patients with positive symptoms had more connections in the temporal areas.

The greatest departure of schizophrenics from the norm was revealed at the frequency 35-40 Hz: In the norm there were many typical interhemispheric connections, while in schizophrenics there were no interhemispheric connections at this frequency. Instead, a new pathological system seems to have evolved in patients - the system of connections at a frequency $(29 \mathrm{~Hz})$ significantly lower than normal.

Conclusions. 1. In both groups of schizophrenic patients there is phase shift instability, revealed by the coherence method. This instability causes functional disconnections between the hemispheres in the beta2-rhythm, this rhythm being important for cognitive functions and consciousness.

2. In both groups of patients, the number of typical connections was decreased in comparison with the norm. In patients with negative symptoms, this number was greater than in the other patient group, but on account of lower frequency connections.

3. All the abovementioned deficiencies observed in schizophrenic patients may be at least partly connected with NMDAhypofunction.

\section{Synchronous dynamics for cognitive coordination: But how?}

\section{M.-A. Tagamets ${ }^{\mathrm{a}}$ and Barry Horwitz ${ }^{\mathrm{b}}$ \\ a Functional Neuroimaging Laboratory, Maryland Psychiatric Research Center, University of Maryland School of Medicine, Baltimore, MD 21227; ${ }^{\mathrm{b} B r a i n}$ Imaging and Modeling Section, NIDCD, National Institutes of Health, Bethesda, MD 20892. mtagamet@mprc.umaryland.edu horwitzb@nidcd.nih.gov}

\footnotetext{
Abstract: Although interesting, the hypotheses proposed by Phillips \& Silverstein lack unifying structure both in specific mechanisms and in cited evidence. They provide little to support the notion that low-level sensory processing and high-level cognitive coordination share dynamic grouping by synchrony as a common processing mechanism. We suggest that more realistic large-scale modeling at multiple levels is needed to address these issues.
}

The main hypothesis advanced by Phillips \& Silverstein $(\mathrm{P} \& S)$ is that synchrony of fast rhythms (e.g., gamma or beta rhythms) can explain "cognitive coordination" throughout the cortex at multiple levels of processing, and that schizophrenia results from a breakdown of this coordination. These dynamics, in turn, are hypothesized to be regulated by NMDA-receptor activity, which is thought to be reduced in schizophrenia. The evidence presented by $P \& S$ mainly derives from studies of context in perceptual processing, and P\&S suggest that the principles at this level can be extended to higher level cognitive processing throughout the cortex. They hypothesize that synchrony of high-frequency rhythms across cell assemblies is sufficient as an explanatory mechanism for a dynamic grouping that underlies cognitive coordination at multiple levels. But is this consistent with the evidence? Furthermore, while the $\mathrm{P} \& \mathrm{~S}$ hypothesis is appealing in its parsimony, it is not clear that synchrony is necessary to explain the phenomena described. In the debate on the role of rhythms in the cortex, it has been argued, including by one of the authors (Phillips \& Singer 1997a), that no rhythmic patterns are necessary for synchronization to occur (Roy et al. 2001).

How appropriate is it to assume a unified process across multiple levels, such as low-level perception and cognitive coordination of high-level processes? In general, there is little explanation in the target article of specific mechanisms that make use of synchrony that might support the authors' view. Rather, supporting evidence is drawn for each of the parts of the argument, but little is done to induce a "Gestalt": How, exactly, do all the pieces fit together? Does "coordination" of perceiving the parts of an object as a whole equate with selecting an appropriate answer to a simple perceptual matching task, and does the latter require the same type of coordination that is needed for playing chess or for daydreaming? If so, then which components of each task are bound by the dynamic grouping, and in what order? Synchronous gamma band activity has been proposed to serve a role in binding components of a percept into a unified whole, and most of the evidence cited by $P \& S$ draws on this literature. This, in itself, is not coordination, in the more common sense of the word. Coordination implies sequences of actions in some useful order in time, and certainly, higher-order cognitive processes require coordination in this sense. No satisfactory definition is given to the term "coordination," and no explanation is given here of how the proposed synchronous mechanisms could mediate the necessary transitions between states for coordination to occur.

A large part of the authors' argument revolves around the role of NMDA both in mediating the synchronous behavior across multiple levels, and in the lack of this coordination in schizophrenia. Yet it is widely believed that dopamine plays a significant role in control of processing in the frontal cortex, but not in early sensory and association regions of the cortex. This fact already suggests potentially different basic mechanisms. With respect to schizophrenia, virtually all antipsychotics have dopamine receptor binding action. Although the evidence for NMDA-receptor involvement in schizophrenia is compelling (Holcomb et al. 2001; Medoff et al. 2001; Tamminga 1998), it is not clear that it alone can explain the disease, since NMDA partial agonists seem to be most effective in treating negative symptoms (van Berckel et al. 1996), and mainly in conjunction with other, more standard antipsychotics. Recent theories of schizophrenia have rather emphasized the likely role of multiple interacting receptor systems in this disease. One theory that might involve the type of dynamics proposed by $\mathrm{P} \& S$ is in studies of the regulation of dopamine by NMDA-receptor activity. Specifically, there is evidence that NMDA-receptor-based mechanisms may regulate the flow of dopamine from the ventral tegmental area (VTA) to the prefrontal cortex (PFC) in a reciprocal interaction which depends on a temporal pattern of activity in the VTA (Svensson 2000).

A bursting pattern of activity at $5 \mathrm{~Hz}$ (near the alpha-rhythm) in the VTA caused dopamine release to the PFC, while nonbursting spiking at the same mean rate did not. This dynamic behavior was found to depend on NMDA-receptor activation by afferents 
from the PFC. Although dynamic interactions are very much a part of this theory, both the qualitative behavior and the temporal scale are inconsistent with the fast-frequency mechanisms proposed by P\&S. Low-frequency rhythms such as alpha are thought to synchronize loosely at best (Kopell 2000). Although on the surface this could be viewed as coordination that is mediated by NMDA-induced temporal patterns of activity, it is difficult to assess whether this scheme would fit into the scheme proposed by $\mathrm{P} \& S$; there is a lack of any specific mechanism in the target article.

The proposal of $\mathrm{P} \& \mathrm{~S}$ emphasizes the critical interplay between computational modeling and experimental observation. Although we agree that combining modeling and experimental observation is essential if one is to begin to understand complex cognitive processes and their dysfunction, we have argued (Horwitz et al. 1999; Tagamets \& Horwitz 1998) that, to understand the neural substrates mediating cognitive tasks (and brain disorders), it is necessary to embed the specific assumptions one makes into a neurobiologically realistic model and to simulate neural data at multiple levels (e.g., single unit, fMRI) that can be compared with quantitative experimental data. In the case presented by $P \& S$, such a model would have defective NMDA-receptors, and one would attempt to show that the model is deficient in performing tasks on which schizophrenic patients show deficits, and also, that the model results in simulated neural activity similar to that seen in schizophrenic patients (e.g., hypofrontality in an appropriate fMRI study). It is at this level of analysis that one can start to determine the neural basis of the disruption of normal cognition that one finds in schizophrenic patients.

The type of hypothesis proposed by $\mathrm{P} \& \mathrm{~S}$ should be viewed as the beginning stage for a neurobiologically realistic physiologic theory aimed at elucidating the neural basis of the thought disorder associated with schizophrenia, not as a theory itself.

\section{ACKNOWLEDGMENT}

M.-A. Tagamets is supported by NIMH K-01 Award No. MH064622-01A1.

\section{Combating fuzziness with computational modeling}

\author{
L. M. Talaminia ${ }^{\text {, M. Meetera }}{ }^{\mathrm{a}}$ and J. M. J. Murre ${ }^{\mathrm{b}}$ \\ a Department of Psychology, Section of Psychonomics, University of \\ Amsterdam, 1018 WB Amsterdam, The Netherlands; ${ }^{\mathrm{b}}$ Department of \\ Computer Science, University of Maastricht, 6200 MD Maastricht, The \\ Netherlands. Italamini@fmg.uva.nl_m@meeter.nl \\ jaap@murre.com http://www.neuromod.org
}

Abstract: Phillips \& Silverstein's ambitious link between receptor abnormalities and the symptoms of schizophrenia involves a certain amount of fuzziness: No detailed mechanism is suggested through which the proposed abnormality would lead to psychological traits. We propose that detailed simulation of brain regions, using model neural networks, can aid in understanding the relation between biological abnormality and psychological dysfunction in schizophrenia.

In the last decades, the sciences studying brain and behavior have started to converge. The paper by Phillips \& Silverstein $(P \& S)$ is a good example of this tendency. In it, the cognitive and behavioral symptoms of schizophrenia are tied to a putative dysfunction of the NMDA-receptor. The vast differences in scale that are bridged in the process are both the strong-point and the weakness of this paper. On the one hand, such an explicit bridge is necessary to link an identified neuropathology to observed cognitive or behavioral impairments in a testable way. On the other hand, because of a shortness of relevant data, the cellular and network mechanisms that might lead from NMDA-receptor dysfunction to the symptoms associated with the disorder largely remain obscure.

Nevertheless, P\&S's viewpoint focuses attention on a thus far poorly understood issue in schizophrenia research, namely, the remarkable similarities between effects induced by NMDA-receptor blockers such as PCP and schizophrenia symptoms. The mechanisms underlying these effects are of considerable importance, both with respect to disorder pathogenesis and in relation to drug therapy. $P \& S$ present various evidence that suggest a role of NMDA-receptors in at least some of the symptoms of schizophrenia. This link is most convincing with respect to deficits on perceptual tasks. The authors discuss in detail what paradigms are affected in this domain, and how deficits in these tasks are related to the concepts of cognitive coordination and dynamic grouping. It is also in this field (in auditory perception) that they present the sole direct evidence linking NMDA-receptor blockage to a decrease in contextual modulation (Javitt et al. 1996).

With respect to the cognitive symptoms discussed in the article, the distance from the neurochemical level is so great that some vagueness is necessarily introduced. In these issues both the step from NMDA to cognitive coordination, and that from cognitive coordination to symptomatology, are not wholly transparent. For example, P\&S argue that NMDA-receptors typically carry information from outside the receptive field of the cell. However, in higher order areas, the notion of receptive field may be problematic. Still, the authors suggest a distinction can be made between what neurons "transmit information about," and information that is merely modulating. It would seem to us that such distinctions are clear-cut only in lower order sensory or motor areas, where the relation between environmental stimuli and neuronal representations is relatively straightforward. How such distinctions are to be applied to, for example, hippocampal structures with their multiple recurrent circuits, is unclear.

Another problem is that the exact nature of the putative NMDA abnormality in schizophrenia remains undefined. NMDA-receptors have been associated primarily with learning (Newcomer \& Krystal 2001). Although the authors show that some symptoms in schizophrenia cannot be caused by LTP deficits, we wonder why learning would not be affected, and how LTP deficits would fit in their story.

At the cognitive level, the concept of "cognitive coordination" is thus used in a rather loose way. It seems that anything and nothing can be a reflection of cognitive coordination. As a consequence, the predictions of the theory at the cognitive level are unclear. This suggests that it would perhaps be more fruitful to consider a link of NMDA-receptors and contextual modulation within the limited domain of sensory tasks. Although this would greatly limit the generality of the theory, it would have the benefit of making it relatively clear-cut what types of transmission are thought to be affected by NMDA abnormalities, and how that relates to behavioral effects. Loss of generality would thus be made up by gains in testability.

In more general terms, the closing of the gap between brain and behavior might benefit from models that are explicit about how an observed neuropathology, in a particular brain region, might affect network function, and how this, in turn, may result in particular symptoms. As stressed by P\&S, simulation of brain circuits using computational techniques can be a great aid in this process, provided that one is explicit about the substrate that is modeled.

There are already several examples of such an approach (Andrew 2000; Braver et al. 1999; Meeter et al. 2002; Monchi et al. 2002). The study by Andrew (2000) showed how dopamine upregulation, simulated as an increase in excitability in frontal cortex neurons, produced perseverance errors on a simulated Wisconsin Card Sort Test, as also found in patients with schizophrenia. As another example, in our own work we relate specific wiring abnormalities in the parahippocampal gyrus with the pattern of memory deficits observed in schizophrenia (Meeter et al. 2002; Talamini et al., in preparation). The architecture of our network incorporates the broad features of medial temporal lobe anatomy, while the simulated neuropathology is derived from a considerable body of evidence that suggests reduced connectivity in the input-output regions of the hippocampus (Harrison \& Eastwood 
2001; Hemby et al. 2002). The model's performance on a number of episodic memory tasks is compared with scores from patients with schizophrenia on the same tasks. Thus, the model is constrained at various levels of architecture and performance, and correspondences between both model and brain, and model and behavior are explicit. This facilitates verification or falsification of hypotheses, while derivation of new predictions can occur in an unambiguous way.

This type of analysis may also be appropriate for studying the role of NMDA currents in schizophrenia, as proposed by P\&S. As the behavioral effects of abnormalities in these currents will probably depend on the wiring architecture of the region, network characteristics in the areas underlying the function of interest will need to be considered. For example, a computational model of auditory cortex may show how NMDA-receptor anomalies could produce deficits in sensory processing. Computational modeling thus allows the investigation of concrete deficits produced by concrete abnormalities in the brain.

\section{Guarding against over-inclusive notions of "context": Psycholinguistic and electrophysiological studies of specific context functions in schizophrenia}

\author{
Debra Titone $^{\mathrm{a}}$ and J. Bruno Debruille ${ }^{\mathrm{b}}$ \\ a'Department of Psychology, McGill University, Montreal, Quebec, H3A $1 B 1$ \\ Canada; ${ }^{\mathrm{b}}$ Department of Psychiatry, Douglas Hospital Research Center, \\ McGill University, Verdun, Quebec, H4H 1 R3 Canada. \\ dtitone@hebb.psych.mcgill.ca bruno.debruille@douglas.mcgill.ca \\ http://www.psych.mcgill.ca/faculty/titone.html
}

Abstract: Phillips \& Silverstein offer an exciting synthesis of ongoing efforts to link the clinical and cognitive manifestations of schizophrenia with cellular accounts of its pathophysiology. We applaud their efforts but wonder whether the highly inclusive notion of "context" adequately captures some important details regarding schizophrenia and NMDA/glutamate function that are suggested by work on language processing and cognitive electrophysiology

Schizophrenia is an enigmatic disorder associated with impairments in the structure and function of many interdependent neurocognitive systems. The neural and cognitive manifestations of schizophrenia are wildly heterogeneous, nevertheless significant advances have been made in understanding its biology and psychology. Phillips \& Silverstein's (P\&S's) thesis builds upon recent advances in linking cognitive dysfunction in schizophrenia to the NMDA/Glutamate system, and we applaud their efforts. However, similar to other comprehensive views of schizophrenia (e.g., Cohen \& Servan-Schreiber 1992), we wonder whether their overinclusive view of "context" adequately captures some important subtleties regarding neurocognitive dysfunction in schizophrenia.

In the domain of language, for example, the channel through which thought disorder in schizophrenia is most apparent, context may affect processing at any number of functional levels, thus clouding the notion of what constitutes a "primary" or "receptive field" input. These levels include the encoding of the auditory speech signal (or printed word), the activation of lexical entries, the retrieval of semantic information, how semantic information gets bound into episodic memory, and so on. Moreover, a given context may exert independent and separable effects in normal language processing that are differentially impaired in schizophrenia patients and following ketamine administration in normals (thus interfering with the NMDA/glutamate system directly). Such examples, to be reviewed, are problematic for global notions of "context" because they suggest that context processing is not unitary but rather a collection of processes, only a subset of which may be impaired in schizophrenia or arise from NMDA/ glutamate function.
Language contexts exert facilitative and inhibitory effects on semantic activation that are differentially impaired in schizophrenia. We examined whether schizophrenia patients differentially use context to facilitate priming of contextually relevant meanings, and to inhibit priming of contextually irrelevant meanings (Titone et al. 2000) using a semantic priming method. Participants listened to moderately or strongly biased sentences that contained ambiguous words (e.g., bug), and saw targets presented for a lexical decision response (i.e., word/nonword judgment) that were semantically related to dominant (e.g., insect) or subordinate (e.g., spy) meanings. Sentence contexts always favored the subordinate, or less frequent meaning; therefore, priming to subordinate targets was always contextually appropriate and priming to dominant targets was always contextually inappropriate.

Similar to the perceptual closure data cited by P\&S, schizophrenia patients differed from controls only for moderately biased but not strongly biased contexts. For strongly biased subordinate contexts, both schizophrenia patients and controls used context to selectively prime subordinate meanings, and thus to appropriately inhibit dominant meanings. For moderately biased subordinate contexts, however, only controls selectively primed subordinate meanings. In contrast, schizophrenia patients significantly primed both meanings, which indicates that they used the context to appropriately enhance subordinate meaning activation but not to inhibit dominant meaning activation (see also Chapman et al. 1976; Done \& Frith 1984 for similar results; see Titone et al. 2002 for evidence of schizophrenia-related priming impairments for ambiguous idioms). These data suggest that context may produce multiple effects simultaneously - the activation of relevant semantic representations and the inhibition of irrelevant semantic representations. Further, these distinct effects of context may be selectively disrupted in schizophrenia.

Distinct ERPs associated with context processing are differentially impaired in schizophrenia subtypes and following ketamine administration. At least two event-related-potential (ERP) components may be relevant to context processing and NMDA accounts of schizophrenia. One deflection, the N400, is evoked by stimuli that are meaningful in everyday life, such as words, sentences, objects, or faces. For most authors, the N400 indexes the integration of stimulus meaning into an unfolding context (Halgren \& Smith 1987; Holcomb 1993; Rugg et al. 1988), or the inhibition of inappropriate knowledge (Debruille 1998; Debruille et al. 1996). A second component, the P600 (a member of the P3b family; Kutas et al. 1977), is also evoked by stimuli that are meaningful in everyday life. Its amplitude is larger for stimuli that are subsequently recalled (Kutas 1988; Neville et al. 1986), and as the amount of information that is consciously extracted from a stimulus configuration increases (Donchin \& Coles 1988). Therefore, the P600 potential may be related to the formation of an episodic memory event, or stimulus "binding."

Within P\&S's framework, both the N400 and the P600 qualify as context-sensitive potentials. The N400 appears to be abnormal in schizophrenia (Koyama et al. 1991; Niznikiewicz et al. 1999; Sitnikova et al. 2002), especially as thought disorder or cognitive disorganization increases (Andrews et al. 1993). P600 amplitude is also smaller in schizophrenia patients than in controls, although schizophrenia patients who frequently experience reality distortion (i.e., hallucinations and delusions) show significantly larger (i.e., relatively more intact) P600 amplitudes than schizophrenia patients who do not experience prominent reality distortion (Guillem et al. 2001; 2003). Thus, the P600 effect is consistent with $\mathrm{P} \& S$ 's interpretation of gamma in schizophrenia, which is also thought to reflect episodic binding and is less impaired in schizophrenia patients who frequently experience reality distortion.

Although these N400 and P600 results fit with P\&S's framework, the available evidence regarding these potentials and ketamine clouds the picture. Ketamine produces significant amplitude reductions in one of the most important sources of the N400, the antero-medial temporal lobe (AMTL) generator (Grunwald et al. 1999). Thus, the N400 findings are consistent with P\&S's notion 
of an NMDA-dependent relationship between cognitive disorganization in schizophrenia and contextual coordination. However, ketamine does not alter the AMTL generator of the P600 (Grunwald et al. 1999). Thus, context functions related to the inhibition of mental representations (i.e., N400) are impaired in schizophrenia, and associated with ketamine-induced MNDA/glutamate system damage. In contrast, context functions related to episodic memory formation and binding (i.e., P600), although impaired in schizophrenia, may not be associated with ketamine induced NMDA/glutamate system damage.

Recommendations. Although the studies reviewed here are preliminary, we hope they serve as examples of why pathophysiological models of schizophrenia should move beyond overly inclusive views of context and context-mediated effects, however tempting they may be. Only by decomposing the specific neurocognitive processes involved in any one "context" task, as well as understanding the interrelationships between critical processes involved across tasks, will the precise relationship(s) emerge between cellular and higher-level cognitive mechanisms that produce the intriguing pattern of cellular and cognitive deficits found in schizophrenia. Given the computational sophistication of P\&S's contextual coordination approach, we hope they continue to pursue more precise linkages between specific context functions, NMDA/glutamate system function, and cognitive processing abnormalities in schizophrenia.

\section{ACKNOWLEDGMENTS}

The first author is grateful for support from the Canada Research Chairs Program, the Canadian Foundation for Innovation (CFI), the National Institutes of Mental Health (NIMH), the National Alliance for Research on Schizophrenia and Affective Disorders (NARSAD), and the McGill University Internal Research Development Fund Committee. The second author is grateful for support from the Natural Science and Engineering Council of Canada (NSERC), the Canadian Institute for Health Research (CIHR) and the Douglas Hospital Foundation.

\section{High-frequency synchronisation in schizophrenia: Too much or too little?}

\author{
Leanne M. Williams ${ }^{a}$, Kwang-Hyuk Lee ${ }^{b}$, Albert Haig ${ }^{c}$, and \\ Evian Gordonc \\ a School of Psychology, (A19), University of Sydney, Camperdown, NSW, \\ 2006, Australia; ${ }^{b}$ Brain Dynamics Centre, Westmead Hospital, University of \\ Sydney, Westmead, NSW, 2145, Australia; 'Brain Dynamics Centre and \\ International Brain Resource Database, Westmead Hospital, University of \\ Sydney, Westmead, NSW, 2145, Australia. lea@psych.usyd.edu.au \\ md4khl@sheffield.ac.uk albert@brainresource.com \\ egordon@mail.usyd.edu.au http://www.brain-dynamics.net
}

\begin{abstract}
Phillips \& Silverstein's focus on schizophrenia as a failure of "cognitive coordination" is welcome. They note that a simple hypothesis of reduced Gamma synchronisation subserving impaired coordination does not fully account for recent observations. We suggest that schizophrenia reflects a dynamic compensation to a core deficit of coordination, expressed either as hyper- or hyposynchronisation, with neurotransmitter systems and arousal as modulatory mechanisms.
\end{abstract}

Phillips \& Silverstein $(\mathrm{P} \& S)$ present a welcome focus on large-scale coordinating mechanisms, as a complement to spatially localised brain functions. From this perspective, schizophrenia deficits in cognitive coordination stem primarily from reduced NMDA activity. The focus on NMDA-hypofunction suggests an associated emphasis on reduced coordination. It is noted that high frequency (40$\mathrm{Hz}$ ) Gamma activity is related to NMDA-receptor activity, as well as to dynamic organisation and coordination. From this context, the authors present the logical first-pass hypothesis that Gamma activity will be reduced in schizophrenia. Although they cite core supporting studies, $\mathrm{P} \& \mathrm{~S}$ also point to observations of abnormally enhanced Gamma activity that suggest a more complex hypothesis.
We have recently reported a series of studies on Gamma activity in schizophrenia (for a review, see Lee et al. 2003b), examining both Gamma power and Gamma phase synchrony. While we observed a consistent reduction in Gamma activity in groups of both chronic and first-episode subjects, a more specific pattern of abnormalities has emerged in relation to distinct syndromes. Disorganisation is associated with the most disturbed profile of Gamma activity across task-relevant and task-irrelevant stimuli. It is associated with widespread increases in synchrony for task-relevant stimuli (Lee et al. 2003c). By contrast, Disorganisation is associated with a reduction in Gamma power frontally for taskirrelevant stimuli (Gordon et al. 2001). An opposing and more spatially localised pattern has been revealed for Psychomotor Poverty (primarily left hemisphere hypofunction) and Reality Distortion (primarily right hemisphere hyper-function) in relation to both Gamma synchrony and Gamma power (Gordon et al. 2001; Lee et al. 2003c).

We suggest that the widespread enhancement of Gamma synchrony with Disorganisation is synonymous with the authors' observation that disturbances in perceptual organisation (reflecting a failure of cognitive 'binding') increase specifically with severity of Disorganisation. The target article does not present specific predictions concerning the other syndromes. We speculate that Psychomotor Poverty and Reality Distortion might represent an attempt to compensate for a core deficit in synchronisation associated with the clinical syndrome of Disorganisation, in terms of either under or over-binding of stimuli. By contrast, Disorganisation might reflect a fundamental inability to locate a state of compensation and is therefore expressed as the most disrupted pattern of binding, to both task-relevant and task-irrelevant stimuli. From this view, the "compensatory" syndromes may be conceptualised in terms of "hyposynchronisation" (distinct reduction in binding and information processing shut-down associated with Psychomotor Poverty) or "hypersynchronisation" (abnormal increase in binding and over-processing associated with Reality Distortion). This speculation is consistent with functional disconnection models of schizophrenia which associate positive schizophrenia symptoms with "hyperconnectivity" and negative symptoms with "hypoconnectivity."

Is such a dynamical compensation view compatible with the NMDA-hypofunction model? We propose a number of interacting mechanisms, consistent with the authors' suggestion that the consequences of both direct and indirect neurotransmitter function may produce the diversity of schizophrenia symptoms. NMDA-hypofunction could account for general hypersynchronisation in Disorganisation, via the effects of glutamatergic activity which has a widespread cerebral distribution (Javoy-Agid et al. 1989). The authors note that NMDA-antagonists such as ketamine have been shown to produce Disorganisation in a dose-dependent manner. Ketamine also produces excessive glutamatergic activity, which in turn leads to enhanced synchronous Gamma activity. Enhanced dopamine activity (associated more closely with Reality Distortion (Oades et al. 1994) has also been related to excessive Gamma activity. Dopaminergic effects appear to be more regionally localised than NMDA-glutamatergic effects, and modulate task-relevant selective attention in particular, consistent with the regionally localised and task-related hypersynchronisation in Reality Distortion (Ahveninen et al. 2000). By contrast, we speculate that hyposynchronisation in Psychomotor Poverty may reflect cholinergic activity, given that anticholinergic agents produce a reduction in Gamma activity, and that clozapine (antipsychotic medication that is a partial cholinergic agonist) improves cognitive deficits that are most apparent in this syndrome (Allen et al. 1993). Bearing in mind the complexity of receptor subtypes, these speculations raise the possibility that abnormal regulation of dopamine or cholinergic activity may represent longer time-scale neuromodulatory attempts to cope with a Disorganisation-related deficit in NMDA activity.

In addition, we suggest that arousal may act as a dynamic regulator of the interactions between these neurochemical effects and 
cognitive coordination. It has been demonstrated that the relationship between synchronous Gamma activity and cognitive coordination is modulated by phasic arousal of specific cortical regions (Sheer 1984). Grossberg (1984) argued that psychosis can be conceptualised as the expression of an opponent process in which arousal is gated by slowly accumulating neurotransmitters. In this case the inverted-U properties of under- and overarousal may be represented clinically as the compensatory syndromes of Psychomotor Poverty (hyposynchronisation, decreased cholinergic modulation) and reality distortion (hypersynchronisation, increased dopamine modulation) syndromes, respectively. P\&S suggest that computational modeling provides an important way forward in elucidating interactions of NMDA activity with other neurotransmitter systems. A numerical simulation of whole-brain neurophysiology developed by our group has demonstrated that enhanced Gamma activity emerges from an increase of gain-related parameters of the thalamo-cortical feedback loop and associated cortical arousal (Rennie et al. 2000). The reliance of this approach on physiological parameters offers a quantitative means to examine neurotransmitter function in relation to synchronous brain activity.

\section{Authors' Response}

\section{Cognitive coordination and its neurobiological bases: A new continent to explore}

\author{
Steven M. Silverstein ${ }^{a}$ and William A. Phillips ${ }^{b}$ \\ a Department of Psychiatry, Psychotic Disorders Division, Weill Medical \\ College of Cornell University, New York Presbyterian Hospital, White Plains, \\ NY 10605; 'bepartment of Psychology, Center for Cognitive and \\ Computational Neuroscience, University of Stirling, Stirling FK94LA, \\ Scotland, United Kingdom. steven.silverstein@att.net \\ wap1@stir.ac.uk
}

Abstract: The additional arguments and evidence supplied by the commentaries strengthen the hypothesis that underactivity of NMDA receptors produces impaired cognitive coordination in schizophrenia. This encourages the hope that though the distance from molecules to mind is great, it can nevertheless be traversed. We therefore predict that in this decade or the next molecular psychology will be seen to be as fundamental to our understanding of mind as molecular biology is to our understanding of life.

\section{R1. Introduction}

The commentaries raise many relevant issues, provide new and important evidence, and offer many valuable insights. The range of disciplines from which the commentators come is a testament to the generality of the issues discussed. Overall, we see them as supporting the general perspective proposed, but asking for further clarification, detail, and evidence. We strongly agree this is needed, as we see cognitive coordination, its neural bases, and their malfunctions, not as a small and well-known territory, but as a vast continent that remains largely unexplored.

Section R2 discusses comments concerning the underlying conceptual framework. Sections R3 to R8 discuss cognitive coordination, and its relation to other aspects of schizophrenia; R9 discusses NMDA hypofunction; R10 discusses the relevance of high-frequency rhythms; R11 discusses the role of computational theory and computational models. Finally, section R12 discusses implications for treatment.

\section{R2. The basic conceptual framework}

\section{R2.1. Terminology}

"Cognitive coordination" refers to the family of interactions that modify neuronal signals without changing what they mean. "Meaning" refers to features detected within perceptual systems, to particular movements within motor systems, and to the semantic contents of thoughts, plans, and memories. We assume that interactions that change the time at which signals occur do not change their meaning. Dysfunctions of timing as discussed by Condray \& Steinhauer are therefore prima facie examples of a disorder in cognitive coordination. Similarly, interactions that affect salience by modulating response amplitude do not change the meaning of the signals that they make more salient. This conception is expressed formally using information theory as cited in the target article. As Grossberg clearly saw, we emphasise two distinct but interdependent classes of cognitive coordination, that is, contextual disambiguation and dynamic grouping. "Context" is activity that affects the processing of signals without changing what they mean. Information theory has been used in psychophysical paradigms designed to distinguish modulatory interactions from those that determine the semantic content of the signals modulated (Phillips 2001; Phillips \& Craven 2000). This is one way of clarifying the core construct of cognitive coordination as requested by MacDonald. In the psychophysical experiments of Phillips and Craven (2000) evidence for driving interactions was easier to find than evidence for coordinating interactions. This should alleviate MacDonald's concern that coordination may be so ubiquitous that it is difficult to test. The central point here is that coordination is identified with interactions between variables, not with tasks as a whole. The importance of such "process-oriented" approaches to the study of cognition and its dysfunctions is discussed in detail by Knight and Silverstein (2001).

We therefore assume that "amplification" and "attenuation" must be distinguished from "excitation" and "inhibition." The former are conditional upon the latter because the latter determine whether there is something to be modulated. Though obvious to many, this distinction is often overlooked.

"Grouping" is the specification of a subset of signals on which to perform some operation. "Prespecified grouping" is that which is specified without knowing the realized values of the variables grouped. In perceptual systems it determines the features to which cells are sensitive. "Dynamic grouping" is that which can be specified only after the realized values are known, that is, after the stimulus has been received in perceptual systems (Watt \& Phillips 2000). "Dynamically embedded grouping" specifies groups within groups, that is, embedded levels of grouping where the embedding cannot be prespecified. Tagamets \& Horwitz argue that "coordination" refers to sequences of actions, and should not be used to refer to perceptual organization. They seem to be in a minority on this, as the other commentators use the term in the way we do.

At the synaptic level, we have used the terms RF (receptive field) and CF (contextual field) to distinguish inputs involved in the two classes of interaction. Local processors 
transmit information about $\mathrm{RF}$ inputs whether there is $\mathrm{CF}$ input or not. Much less information is transmitted about $\mathrm{CF}$ inputs, and that is conditional upon the RF input. Bressler, Talamini, Meeter \& Murre (henceforth Talamini et al.), and Titone \& Debruille all show that our use of this terminology can be misleading. The classical RF was originally defined as the regions in distal visual space within which small spots of light increased or decreased cellular activity. This notion has since undergone several revisions, including generalization to meaning whatever features a cell detects. We further generalized this terminology, using it to refer to whatever it is that cells transmit information about, contrasting that with contextual inputs. To some commentators, the origin of the term RF within the study of perceptual systems carries the connotation of a concrete reference to present stimulation. As what we intend is more general, some other terminology might be better. Roelfsema \& Supèr contrast “driving” with contextual or modulatory connections. As that might be a better terminology, we use it below.

Roelfsema \& Supèr suggest that hallucinations may result from too much contextual modulation, rather than too little. They may be right, but there is another alternative. The distinction that we make between driving and modulatory connections is an idealized dichotomy. We assume that, in reality, synaptic connections between cells can be a combination of both, with the balance varying across cells. The co-localization of NMDA and non-NMDA receptors is anatomical evidence for this assumption. This balance can also vary dynamically. If cells have low activity for a long time, for example, as a consequence of NMDA hypofunction, then the balance of their inputs can shift such that they become more driving (Das \& Gilbert 1995), thus restoring some functionality to the cell. So, even if hallucinations do arise from increased feedback, as Roelfsema \& Supèr suggest, then that could be due to the feedback's becoming increasingly driving, as a consequence of NMDA hypofunction, rather than through an increase in the activity of NMDA channels.

These arguments for generality do not imply that all cortical interactions are coordinating as Bressler suggests. Many interactions are driving and determine the semantic content of signals. This is particularly obvious in perceptual systems, where the features detected at each level of the hierarchy depend upon how they are driven by input from lower levels. Similarly, PFC and basal ganglia (GoldmanRakic et al. 2000) are composed of multiple parallel streams of processing, each dealing with information about different things but interacting at each stage of processing. This suggests that processing from one stage to the next within streams depends upon driving connections, and interactions between streams on contextual connections.

\section{R2.2. Is cognitive coordination relevant to higher cognitive functions as well as to perception?}

Ginsberg questions the generalization of cognitive coordination to language perception and production. Talamini et al. suggest that it may be more fruitful to limit the concept of contextual modulation to sensory tasks. Titone \& Debruille note that the notion of a receptive field is unclear outside of perception, and emphasize the need to avoid over-inclusive notions of "context."

Suggestions that we should limit the notion of cognitive co- ordination to basic perceptual functions are understandable given the origin of the concept of a receptive field within studies of perception. Nevertheless, we see cognitive coordination as even more relevant to higher cognitive functions. Contextual disambiguation and dynamic grouping are more relevant to higher functions, not less. ${ }^{1}$ NMDA receptors are more dense in higher cortical regions, not less. Higher functions are more impaired in psychoses, not less. Our emphasis on perceptual processes therefore makes the case in a functional domain where, prima facie, it is less likely.

Anatomical, physiological, and psychological evidence for cognitive coordination as a theme common to cortical activity as a whole was reviewed in detail by Phillips and Singer (1997a). In addition, there are general arguments suggesting that diverse cortical activities should not be independent, but coordinated. Coordination is necessary to acquire and use knowledge of predictive relationships, to select activity relevant to the current context, to combat noise, to make coherent choices, and to organize activity into coherent subsets. This applies to cognition in general, not just to perception.

\section{R2.3. Are there different varieties of cognitive coordination?}

Hemsley shows clearly that forms of context may differ in various ways: temporal versus spatial; inhibitory versus facilitatory; tonic versus phasic; and contextual priming versus top-down executive control. Javitt distinguishes between NMDAR-dependent and NMDAR-independent forms of cognitive coordination. Titone \& Debruille distinguish between facilitatory and inhibitory contextual effects, and between contextual encoding (as reflected by $\mathrm{N} 400$ ) and episodic binding (as reflected by P600). An obvious way to distinguish contextual effects is by the domain to which they apply. Chen relates them to integration across space; Titone \& Debruille and Condray \& Steinhauer to language; Raffone, Murre \& Wolters (henceforth Raffone et al.) to working memory, and Leiser \& Bonshtein to theory-of-mind. Park, Lee, Folley \& Kim (henceforth Park et al.) distinguish between perceptual context (unattended aspects of the target, and surrounding items in space and time), cognitive context (long term memory [LTM] and task-relevant information in working memory [WM]), and socio-affective context. Grossberg distinguishes between coordination within the "what" and "where" streams. Last, but not least, Sass \& Uhlhaas argue for forms of context that are relevant to phenomenology.

All of these distinctions may be important. Emphasis upon a general theme in no way implies the absence of variations. Themes and variations are ubiquitous throughout biology. Indeed it is because we think that there are so many variations to be discovered that we see cognitive coordination, not as something simple, but as a continent to explore.

MacDonald views our perspective as denying the importance of the distinction between "top-down" and "bottom-up" coordination. Not so. We do not claim that the same lesion produces deficits in both, but that both may be affected by the same kind of dysfunction. NMDA receptors are involved in both kinds of interaction, but they depend upon different neural pathways and different synapses. Dysfunctions affecting one sub-set but not the other are possible, particularly if they use different sub-types of NMDA receptor. $^{2}$ 
Response/Silverstein \& Phillips: Convergence of perspectives on cognitive coordination in schizophrenia

\section{R3. Is cognitive coordination impaired in schizophrenia?}

There was general agreement among commentators that the data on perceptual organization impairments in schizophrenia are evidence of a failure of coordinating mechanisms. However, Chen finds these results inconclusive, and the evidence for an impairment inconsistent. Park et al. note that whether or not a context-processing deficit is demonstrated in the laboratory may depend on a number of factors. Hemsley and Park et al. point out that there are a number of forms of context processing and that there may not be a unitary form of context processing deficit. Condray \& Steinhauer raise the issue of whether a timing mechanism needs to be added to the processes we described in order to account for language processing impairments in schizophrenia. Titone \& Debruille question whether the view of cognitive coordination we propose is relevant to language processing. Javitt highlights the compatibility of data on the mismatch negativity (MMN) component of the event-related potential as a form of cognitive coordination, and stresses the importance of NMDA receptor hypofunction in accounting for its impairment in schizophrenia. Raffone et al. highlight the importance of coordinating mechanisms for visual working memory. Leiser \& Bonshtein suggest that data on Theory of Mind (ToM) task performance in schizophrenia is consistent with our view. These comments raise several important issues.

\section{R3.1. Is perceptual organization impaired in schizophrenia?}

Chen offers a misleading picture of perceptual organization in schizophrenia. While he is technically correct that some studies demonstrate abnormalities and some do not, these differences are easily reconciled within a meaningful framework. As we have noted (in the target article, and in Knight \& Silverstein 1998 and Silverstein et al. 2000), all studies in which strong top-down input was required for perceptual grouping to occur have found that schizophrenia patients demonstrated impairments (e.g., Silverstein et al. 1996a; 1996b; 1998a). In contrast, when the stimuli to be processed consisted of closed, geometric forms consisting of visual primitives (e.g., Chey \& Holzman 1997; Knight \& Silverstein 1998), or even when these forms consist of noncontiguous elements but the overall shape was a "good form" (Silverstein et al. 1998a; 1998b), patients performed relatively normally. We view these and similar results as indicating that, when processing mainly relies on prespecified feature hierarchies, patients perform relatively normally, whereas when dynamic grouping is involved, performance deteriorates. As a whole then, the data on perceptual grouping are highly consistent with our view.

\section{R3.2. Which cognitive impairments can be usefully conceptualized as being failures of cognitive coordination?}

Hemsley and Park et al. both point out the different forms of context processing that have been described in the literature. We believe that despite the outward differences, there are common mechanisms subserving these different forms of context processing. We have argued, for example, that spatial context and temporal context effects are medi- ated via similar mechanisms ${ }^{3}$, and it is not unreasonable to suppose that context defined by information in WM overlaps with aspects of task or environment context during task performance. It is clear that further work is needed to determine the nature of the relationships, if any, between these forms of context processing. We consider it unlikely, however, that each involves a modulatory mechanism that is independent of the other; and so we again note the utility of an approach to viewing context that begins by distinguishing between driving input and target stimuli, on the one hand, and modulatory input that modifies the timing, salience, and/or perceived behavioral significance of stimuli, on the other.

Difficulties are necessarily encountered when trying to formulate a theory that encompasses functions as diverse as perception and language. Our use of the RF-CF distinction clearly reveals our predisposition to think in terms of perception, and Condray \& Steinhauer and Titone \& Debruille raise the issue of the appropriateness of our view for understanding language processing. Rather than beginning by trying to find a definition for context processing that fits the terminology used to study language (as implied by Titone \& Debruille), we think it is useful to begin with the converging evidence that impairments in perceptual organization and other forms of context processing have been consistently linked to disorganized thinking and speech in schizophrenia patients (e.g., Barch et al. 1999; Izawa \& Yamamoto 2002; Knight \& Silverstein 1998; Kuperberg et al. 1998; Silverstein et al. 1998a; 2000). While this suggests the possibility of a common mechanism, it does not prove one. However, other evidence also suggests a link might be possible. Condray \& Steinhauer suggest that oscillatory activity within the gamma range, which we propose reflects the forms of cognitive coordination we discuss, is also involved in binding information during semantic memory formation and lexical access. This suggests that similar neurophysiological mechanisms may underlie both spatial and temporal integration.

Condray \& Steinhauer mention the importance of a timing mechanism in language processing. We note in the target article that subcortical structures such as the thalamus and basal ganglia may provide such a timing function, and that they may be important in coordinating cognitive activity. Evidence in support of this comes from findings of abnormalities in NMDA receptor subunit expression in the thalamus and hippocampus among schizophrenia patients (Gao et al. 2000; Ibrahim et al. 2000; Smith et al. 2001).

Finally, the recently proposed Oscillator-based Associative Recall model of Brown et al. (2000) provides a nice example of how oscillatory and timing activities can be integrated, and how dynamic context-based models (involving internal context-states and precise timing functions) can be applied to a wide range of phenomena, including perception, short-term memory, and speech production. ${ }^{4}$

Just as our effort to include language processing abnormalities in schizophrenia under the heading of cognitive coordination failures met with some questions, our efforts to apply the concept of cognitive coordination beyond visual perception into memory, working memory-based context processing, and sensory gating phenomena also met with calls for accommodation. A separate discussion of MMN, PPI (prepulse inhibition), P50, and inhibition can be found in section R4. Here, we consider the question of whether context defined by task information held in working mem- 
ory should be included within our view of cognitive coordination.

We agree with MacDonald's call for evidence regarding the covariance of performance on tasks involving context as defined by Cohen and colleagues (Cohen \& ServanSchreiber 1992; MacDonald et al. 2003: Servan-Schreiber et al. 1996), and context as we define it. We are currently collecting such data. Other evidence already suggests that common mechanisms are operative. For example, as Javitt notes in his commentary, ketamine produces impairments on both the (pre-attentive) MMN and (post-attentive, WM based) AX-CPT paradigms. Also, Desimone and colleagues have demonstrated that attentional (top-down) and stimulus driven (bottom-up) effects in V4 operate via similar mechanisms (e.g., Reynolds \& Desimone 2003; see also sect. 2.1 in target article).

While we await further evidence on this issue, we raise the question: Does available evidence justify limiting the conception of context to one involving information held in working memory, or does it suggest that a broader definition of context is more appropriate? We do not see any a priori reason to limit the definition of context to information held in working memory. Common sense dictates that context can be defined using multiple criteria, while still having the same modulatory effects, and this was noted by Hemsley and Park et al. Furthermore, our theory predicts the findings from WM-based tasks, whereas the WM memory model of Cohen and Servan-Schreiber cannot account for the perceptual grouping effects we describe. Moreover, we demonstrated in the target article how our theory of reduced cognitive coordination in schizophrenia can account for a range of phenomena, including perceptual organization deficits and disorganized symptoms. In contrast, the Cohen and Servan-Schreiber model initially predicted (based on the assumption of reduced PFC dopamine) a relationship between their context processing deficits and negative symptoms, but data from subsequent studies indicated a relationship instead with disorganized symptoms, as we would have predicted. We see the consistent findings of relationships between disorganized symptoms and performance on diverse tasks, which we view as exemplifying cognitive coordination (e.g., perceptual organization, lexical disambiguation, working memory-based sustained attention, etc.), as strong evidence for the view we propose. If the definition of context processing is to be limited only to one involving working memory (i.e., temporal context), then any model based on this theory would have to account for the relationships between disorganized symptoms and the other types of task performance which we consider to involve context processing. Such a model would also have to address the views of Park et al., and Hemsley, as well as others in field of cognitive neuroscience (e.g., Gilbert et al. 2000; Raizada \& Grossberg 2001), who clearly see context as involving more than working memory.

Finally, recent evidence demonstrates a bridge between views of context defined by temporal or spatial information. Izawa and Yamamoto (2002) demonstrated a perceptual organization deficit in schizophrenia patients using a task that involved sequential perception of stimulus fragments. In this task, which required integration over both space and time, performance was, as in past studies of perceptual organization, related primarily to the level of disorganized symptoms. In the Silverstein et al. (1998a) study, performance involved an evolving recognition of which rapidly presented dot patterns were repeating and which were new. In that study, schizophrenic performance differed from controls both in terms of their ability to benefit from stimulus repetition (presumably reflecting the strength of the memory representation) and their ability to rapidly recognize repeating stimuli with weak configural properties (suggesting a need for greater exposure to such stimuli before a WM representation that can guide task performance is developed). Also in that study, degree of memory representation formation for nonconfigural stimuli over time was related primarily to disorganized symptoms. These data suggest, at least, that when perceptual organization is evaluated using both temporal and spatial manipulations, the results are similar to those found in studies using only spatial context. These results are consistent with the commentary of Raffone et al., who point to the importance of NMDA receptor mediated coordination in short term memory, and with findings that perceptual organization deficits and short-term visual memory deficits are found among the same subtype of schizophrenia patient (Knight 1984; Knight \& Silverstein 1998). On balance then, we would say that the evidence favors a more inclusive view of context processing.

\section{R3.3. Is the hypothesis of impaired cognitive coordination relevant for understanding theory of mind deficits in schizophrenia?}

We agree with Leiser \& Bonshtein that ToM may be relevant to our theory of cognitive coordination failure in schizophrenia, in that ToM requires the ability to coordinate several pieces of information at once. Other evidence in support of this position comes from experimental work in autism, where weak central coherence has been associated with poor ToM performance (Jarrold et al. 2000). Jarrold et al. postulate that ToM is a high level manifestation of central coherence, the latter being a concept that has significant overlap with cognitive coordination, but without specification at the computational or neurobiological levels. Jarrold et al.'s view is similar to that of Sarfati et al. (1997), who conceptualized ToM impairment in schizophrenia as being due to a failure to process context, and linked ToM deficits to disorganized symptoms, as our theory would predict (Sarfati et al. 1997). Data we have collected also support a link between ToM deficits and cognitive coordination failures. For example, Schenkel et al. (2001) observed a -.59 correlation between ToM performance (i.e., scores on Corcoran et al.'s [1995] Hinting Task) and the Cognitive/ Disorganization factor of the Positive and Negative Syndrome Scale (PANSS).

More recently, we observed correlations between Hinting Task scores and Spaulding et al.s (1999) Brief Psychiatric Rating Scale (BPRS) Psychotic Disorganization factor among long-hospital-stay schizophrenia spectrum patients $(\mathrm{N}=40, \mathrm{r}=-.31, \mathrm{p}=.05)$ and among a mixed group of inpatient and outpatients with schizophrenia $(\mathrm{N}=36, \mathrm{r}=$ $-.73, \mathrm{p}<.001)$. Also, scores on the Hinting Task correlated $.36(\mathrm{p}<.05)$ with contour integration test (Silverstein et al. 2000) scores among the long-stay group (Schenkel et al. 2001), and $.31(\mathrm{p}=.08)$ among the mixed group. Among the mixed group of patients, BPRS Psychotic Disorganization scores correlated significantly with poor premorbid social functioning, linguistic context processing, and contour integration performance (Schenkel \& Spaulding 2002). Fi- 
nally, Uhlhaas (2003) found that schizophrenia or schizoaffective disorder patients with disorganized symptoms performed more poorly on ToM tasks, and better on a visual context processing task where intact context processing hinders about target stimuli, compared to patients without disorganized features. We view all of these data as consistent with Leiser \& Bonshtein's suggestion that ToM is a form of cognitive coordination.

\section{R3.4. Cognitive coordination impairments and the symptoms of schizophrenia}

No discussion of the question of whether cognitive coordination is impaired in schizophrenia would be complete without a discussion of symptomatology. In general, there was little discussion of our view, and data, indicating that disorganized symptoms represent a "molar" manifestation of reduced cognitive coordination, exemplified in more "molecular" form in laboratory demonstrations of dysfunctions of auditory and visual perceptual organization and other forms of context processing. Williams, Lee, Haig \& Gordon (Williams et al.) provide EEG evidence consistent with our view, by demonstrating relationships between gamma frequency abnormalities and disorganization in schizophrenia. They also go beyond our focus on disorganization and provide evidence that positive and negative symptoms may represent compensatory reactions to impaired coordinating processes. The whole brain modeling approach of the Williams group, including simultaneous recording of EEG, fMRI, cognitive, and arousal indices, represents a powerful technological advance that can be applied to testing the issue of the covariance of multiple forms of disorganization (e.g., cognitive, neurophysiological), including symptoms.

\section{R4. Is our perspective relevant to an understanding of sensory gating, sensorimotor gating, and inhibition deficits?}

Kisley \& Davalos question our inclusion of sensorimotor gating (PPI) and sensory gating (P50) phenomena within the concept of cognitive coordination, noting that there is no convincing evidence that either NMDA receptor activity or gamma frequency oscillations are involved in either. Gooding \& Braun assert that "the cognitive coordination deficit described by $P \& S$ does not adequately capture the cognitive signature of schizophrenia." These comments raise the question of whether we were too inclusive in the range of phenomena we tried to account for by our theory of cognitive coordination reduction in schizophrenia.

\section{R4.1. Sensory and sensorimotor gating}

A number of studies have indicated that NMDA antagonists disrupt sensorimotor gating (PPI) in rats (see Geyer et al. 2001 for a review). However, van Berckel et al. (1998), Duncan et al. (2001), and Oranje et al. (2002), using various stimulus parameters and different doses of ketamine, did not find any effects on PPI in humans. The data on sensory gating (P50 suppression) are also mixed. As noted by Kisley \& Davalos, while early evidence suggested a link between reduced gamma activity and P50 deficits in schizophrenia, more recent work has suggested a greater role for lower frequency cortical rhythms in the generation of the normal P50 suppression effect. In addition, gamma activity in response to the conditioning stimulus appears to expire prior to onset of the test stimulus, making it difficult to see how it could be involved in coordinating the within-pair activity.

Our theory is not wedded to gamma oscillations, however. As we noted in section 5 of the target article, lower frequency rhythms may also be relevant. Van Berckel et al. (1998) and Oranje et al. (2002) indicate that ketamine does affect P50 suppression in humans, but that it does so by reducing responsivity to the conditioning stimulus, without having a significant effect on the test stimulus. These data suggest that sensory and sensorimotor gating phenomena may not involve the type of NMDA receptor mediated coordinating mechanisms we discuss. A critical question, then, is whether these phenomena involve coordinating mechanisms that are different from those we discuss, or whether they should more correctly be viewed as not involving coordinating mechanisms. Recent evidence calls into question whether P50 suppression involves coordination. For example, Blumenfeld and Clementz (2001) and Clementz and Blumenfeld (2001) found that the smaller P50 conditioning-test stimuli ratios in schizophrenia were due to smaller than normal responses to the conditioning (first) stimulus. They suggested that these data were inconsistent with the concept of "poor suppression." Perhaps then, what has been called a P50 suppression abnormality involves not coordination between two paired stimuli, but alterations in a more basic aspect of processing sensory driving input (that is affected similarly by ketamine and schizophrenia). Interestingly, recent evidence also indicates that in healthy adults, responses to the test (second) stimulus can be altered by expectancy and attentional (topdown effects) (Clementz et al. 2002). This further calls into question the idea that P50 suppression reflects a preattentive sensory gating mechanism. In short, newer studies suggest that P50 suppression may not be an example of cognitive coordination, and therefore, in future work this phenomenon may be best conceptualized from within a different perspective. The situation regarding PPI is less clear. It must be kept in mind, however, that all of the PPI studies cited examined acute effects of ketamine. The longterm effects of NMDA receptor hypofunction on PPI still need to be explored.

The evidence for NMDA effects on MMN is much stronger, and is cited in the target article. A recent study supports our view that MMN is a strong example of cognitive coordination. Umbricht et al. (2003) demonstrated that, whereas the serotonin agonist psilocybin disrupted AX-CPT performance in healthy adults, it did not alter MMN activity. Taken in combination with their earlier demonstration that ketamine disrupts performance on both tasks (and that schizophrenia patients perform abnormally on both), Umbricht et al. concluded that whereas impaired AX-CPT performance may result from secondary pharmacological effects shared by both psilocybin and ketamine, ${ }^{5}$ deficient MMN generation in schizophrenia may be a relatively distinct manifestation of NMDA receptor hypofunction (see also commentary by Javitt for further supporting evidence).

Perhaps the simplest conclusion that can be reached regarding these data is that not all forms of cognitive coordination involve the cognitive and/or neurophysiological mechanisms we discuss. This is consistent with Javitt's con- 
Response/Silverstein \& Phillips: Convergence of perspectives on cognitive coordination in schizophrenia

clusion that, while some forms of coordination rely primarily on NMDA receptor activity, others do not. This conclusion raises the following important questions: (1) Is there more than one form of cognitive coordination, and if so, how they are they similar, how do they differ, and to what extent are they independent of each other? and (2) how will the answers to these questions inform our understanding of schizophrenia?

\section{R4.2. Inhibition}

Regarding the general issue of inhibition, which Gooding \& Braun state our theory cannot accommodate, available evidence indicates that the neurophysiological mechanisms we discuss can account for both coordinating and inhibitory effects. One reason for this is that NMDA receptors are involved in projections onto inhibitory neurons, and have strong effects there. For example, NMDA receptors involved in projections onto GABAergic (inhibitory) interneurons are 10-times more sensitive to blockade by NMDA antagonists than are NMDA receptors involved in projections to excitatory neurons (McCarley et al. 1996). A result of reduced NMDA receptor activity is thus reduced inhibition. A consequence of reduced NMDA activity on inhibitory interneurons is a reduction in synchronized gamma activity (Kwon et al. 1999), which we hypothesize to be involved in cognitive coordination. Gamma range activity has been implicated in a number of tasks of perceptual organization (Keil et al. 1999; Lutz 2002) and in other forms of stimulus integration that we believe operate via the cognitive coordination mechanisms we outlined. Furthermore, in post-mortem studies of schizophrenia patients, elevated levels of $\mathrm{N}$-acetyl-aspartyl-glutamate have been observed, and this compound has been observed to preferentially block NMDA receptors mediating excitatory input on inhibitory interneurons (Tsai et al. 1995). Thus, a number of sources of evidence converge to suggest that failures in the neurophysiology underlying coordinating activity (e.g., NMDA receptor activity) can produce reductions in gamma synchrony and inhibition.

We also wish to note that a predicted result of reduced synchronization is the relative weakening of the salience of what should normally be considered "figure" and the subsequent relative increase in "ground" elements (or elements from what Sass \& Uhlhaas, following Gurwitsch, call the margin) in consciousness. Thus, reduced inhibition may be a secondary effect of the lack of a clearly defined focus on conscious awareness. As noted by Hemsley, abnormalities in inhibition and facilitation have been found in schizophrenia, suggesting that both impairments may reflect abnormal modulatory activity.

Contrary to the claim by Gooding \& Braun then, our theory can accommodate evidence of inhibitory failures in schizophrenia. That said, an important area for future efforts will be to extend our theory to account more specifically for the types of inhibitory failures seen among schizophrenia patients in laboratory tasks and during everyday behavior. Just as paradigms that would seem to involve similar mechanisms may not do so (e.g., MMN and P50), there may be forms of inhibitory failure in schizophrenia that do not involve the coordinating mechanisms we discuss, and clarification of this will help improve our theory as well as other theories of schizophrenia.

Finally, an important consideration for theory develop- ment is the relative specificity of the above phenomena to schizophrenia. For example, sensory gating deficits have been observed in mania (Franks et al. 1983), whereas perceptual organization impairments have not been observed in other psychiatric disorders (and sometimes only infrequently among good premorbid schizophrenia patients). These data add the further consideration to theory development that in the search for what is unique about schizophrenia, or about a subtype of this illness, it will be important to identify mechanisms that may be associated generally with psychotic disorders (or mood disorder subtypes with the potential for psychosis), versus those that are associated only with schizophrenia, and then to determine the effects of these multiple abnormalities on the clinical presentation of schizophrenia.

\section{R5. Can reduced cognitive coordination account for the emergence of hallucinations and delusions?}

Hallucinations and delusions are two of the most striking characteristics of schizophrenia, and we are not surprised that so many commentators raised the issue of how the coordinating mechanisms we discuss might be relevant to these symptoms. In response to Alpert \& Angrist, we note again that chronic ketamine effects provide a better model of schizophrenia than acute effects, and auditory hallucinations are common, and more prevalent than visual hallucinations, in cases of chronic ketamine administration. These and similar data have led to widespread agreement on the utility of the PCP-psychosis model of schizophrenia. Bressler's suggestion that reduced coordination could lead to both degradative and illusory phenomena provides a potentially important insight regarding hallucinations that needs to be followed up in future studies. Anecdotal reports from patients suggest that Bressler's hypothesis may also be relevant for delusion formation, as exemplified in the following statement, cited by Mattusek (1952/1987): "Out of these perceptions came the absolute awareness that my ability to see connections had been multiplied many times over" (p. 96). Such anecdotal reports are consistent with Hemsley's position that delusions result from stimuli being evaluated within inappropriate, often idiosyncratic, internal contexts.

Hoffman \& McGlashan take a different approach on this issue, noting how reduced connectivity can lead to "parasitic foci" (Hoffman \& McGlashan 1993) or spurious attractor states wherein hallucinations and delusions form and become relatively fixed phenomena. Bressler's and Hoffman \& McGlashan's varying perspectives highlight the fact that we are still far from agreement about the cause of hallucinations. We are encouraged that both views are consistent with the idea of reduced cognitive coordination, and further tests may clarify processes leading from reduced coordination to the experience of hallucinations. Both of their views are consistent with recent work of Lutz (2002), who studied healthy subjects completing an object perception task, as they moved from states of unreadiness to readiness (and as their EEG records demonstrated increasing gamma band synchronization). Lutz hypothesized that, in the rapid reorganization of mental contents that takes place during the transition from unreadiness to readiness, elements of neural networks involved in the contents of the earlier state 
of consciousness could still be resonating and could be reenslaved into the newly formed assembly. This is a possible mechanism for how, among many schizophrenia patients, where consciousness is not characterized by strong themethematic field links, irrelevant information may become integrated into ongoing thought and action, in some cases even in the form of hallucinations.

Grossberg, and Roelfsema \& Supèr suggest that hallucinations may result from a deficient coordination of bottom-up and top-down feedback. Their views also highlight the importance of viewing coordination within the context of other factors, such as affect, arousal, and significance evaluation. The EEG findings cited by Titone \& DeBruille also highlight the need to clarify whether hallucinations result from excessive sensory input, or from the presence of input which is just not normally allowed access to consciousness, but gains access when conscious awareness is not coherently organized around a specific theme. Relevant to this issue are comments by Sullivan (1956) that, while schizophrenia is characterized by an entry into consciousness of material that would normally be excluded, it is the inclusion of affect laden, "primitive" material that is especially associated with schizophrenia. This view suggests that some material is more likely to impinge on consciousness than others, and that it is the nature of the impinging material that may heighten the state of arousal. This is mere speculation at present; however, two types of data are consistent with it. First, there are excessively high rates of child abuse reported in the histories of psychiatric inpatients with hallucinations (regardless of whether the diagnosis is schizophrenia), and among patients with schizophrenia (Read et al. 2001), suggesting the presence of strong, often dissociated affect states. Second, there is evidence from the rat literature that when early development takes place in a deprived environment, NMDA receptor expression and, presumably, the potential for appropriate coordinating activity is reduced (Liu et al. 2000). One result of developmentally reduced NMDA receptor expression is reduced sensory input, which, in humans, is a potential cause of alterations in the balance of bottom-up and top-down activity. It is thus possible that under conditions of stress, excessive input from highly affectladen memory traces may lead to the emergence of hallucinations in a system that is already imbalanced.

Ginsberg stresses the need to account for the co-occurrence of disorganization with other syndromes. This is important because, while factor analytic studies consistently identify a unique disorganization syndrome, overlap with other syndromes is not zero, and in some cases, symptoms load on more than one factor (e.g., hallucinations loading on both the positive and cognitive/disorganized factors in Lindenmayer et al. 1994). The discussion above points to the possibility that certain positive symptoms can be accounted for within the perspective we propose. It is also possible, however, that symptoms such as hallucinations and delusions are not primarily examples of reduced coordination, but represent compensatory strategies. Williams et al.'s data provides support for this position, which is also consistent with the early work of Bleuler, who viewed hallucinations and delusions as secondary phenomena resulting from a core disturbance in loosening of associations (cf. Bleuler 1911/1961). Related to this question are the unexplored issues of whether cognitive coordination ability exists on a continuum within the general population, and may therefore be a modifying influence for the development of schizophrenia (in that, if one has poor coordination and develops schizophrenia, disorganization is prominent), or whether it is a core aspect of the illness itself.

\section{R6. Is our perspective consistent with developmental views of schizophrenia?}

Gooding \& Braun claim that our theory does not pay sufficient attention to factors that lead to the development of schizophrenia, and write that our perspective "is distinctly lacking in a behavioral genetics perspective." Hoffman \& McGlashan note that our focus on context processing impairments is consistent with their data and with computational models on a neurodevelopmental abnormality involving excessive synaptic pruning. They also note that the sort of local-local connectivity loss we describe is most likely to occur in adolescence, whereas distant connections between brain regions are less likely to be affected by excessive pruning during adolescence. Condray \& Steinhauer agree with our understanding of schizophrenia as a neurodevelopmental disorder, and one that may share features with other such disorders. They suggest that problems during crucial developmental periods, such as in the migration of cortical neurons during prenatal development, and/or in synaptic pruning during adolescence, may interfere with the development of synchronization (resonance) during stimulus processing, and therefore lead to fragmentation in multiple domains. Below, we address two issues related to the developmental course of schizophrenia: Onset and course of illness.

\section{R6.1. Onset of illness}

We see the hypothesis of a cognitive coordination dysfunction as entirely consistent with a neurodevelopmental view of this illness. Several points made in the target article highlight this view, and are consistent with the observations of Hoffman \& McGlashan and Condray \& Steinhauer, including: (1) consistent evidence of links between poor cognitive coordination and the poor premorbid subtype of schizophrenia, suggesting that impaired coordination may premorbidly manifest itself only in more complex situations such as social interactions, and then, with further clinical deterioration, be evident in more basic functions such as perception, thought, and language; (2) the overlap of our view with the theory and data of Hoffman \& McGlashan, suggesting the potential involvement of pruning-related connectivity loss as a potential cause of cognitive coordination reduction; and (3) the presence of cognitive coordination failures, including disorganized symptoms, in other neurodevelopmental disorders, such as autism and some nonverbal learning disabilities.

A critical question for future research involves whether changes on laboratory measures of cognitive coordination precede clinical signs and symptoms, occur simultaneously with them, or occur later. Data on this issue would allow for more refined theorizing about how cognitive coordination failures give rise to psychotic episodes in schizophrenia, and how and why the severity of these failures waxes and wanes over time.

Gooding \& Braun question the relevance of our hypotheses for behavior genetic studies. We find this comment curious, especially in light of the evidence which they believe meet criteria for a behavior genetics approach (see 
Response/Silverstein \& Phillips: Convergence of perspectives on cognitive coordination in schizophrenia

below, next paragraph). Our concept of impaired cognitive coordination is useful in this regard for several reasons. (1) It links biology and behavior. (2) It is rooted in much evidence on the neurobiology and cognitive impairments in schizophrenia. (3) Its most global behavioral manifestation (i.e., disorganized symptoms) has been found to be highly heritable in twin studies of schizophrenia (Cardno et al. 2001). (4) Disorganization among high-risk individuals was found to be one of the few significant predictors of the development of diagnosable schizophrenia within a 12-month period (Yung et al. 2003). (5) Context processing deficits have been found among unaffected siblings of schizophrenia patients (MacDonald et al. 2003). (6) Reduced EEG coherence, which was interpreted as reflecting reduced synchronization, has been found among unaffected siblings of schizophrenia patients (Winterer et al. 2001). (7) Impairments consistent with our view have been linked to a distinct subtype of schizophrenia (patients with poor premorbid social functioning) that is thought to be particularly high in genetic loading for schizophrenia. (8) Mice that have been genetically altered to express only $5 \%$ of the obligatory NMDAR1 subunit of the NMDA receptor demonstrate social deficits (Mohn et al. 1999).

Gooding \& Braun cite a number of their own studies as examples of research useful in clarifying the nature of the development of schizophrenia. These studies are examples of the psychometric high-risk approach, in which selfreport questionnaires that assess Meehl's (1962) behavioral manifestations of schizotypy (thought to reflect the underlying neural integrative defect that he called schizotaxia) are given to young adults (typically college students). High scorers on these scales are thought to be at high-risk for schizophrenia, and are studied further by giving them various tasks to perform to see whether they perform like patients. There are several problems with this approach. For example, by defining the state (schizotypy) solely in terms of behavioral indicators, and neglecting other levels of analysis in the choice of independent variables, the likelihood of generating enough data to develop a comprehensive theory of schizophrenia is reduced. Stated differently, although the psychometric high-risk approach grew out of Meehl's seminal work, its proponents have done little to clarify the nature of the neural integrative deficit, instead focusing on behavioral manifestations of schizotypy and its correlates. Perhaps this is why, after many years of research, the evidence indicates that high scorers on the schizotypy scales rarely progress to develop schizophrenia (Chapman et al. 1994), that many of the deficits found among high scorers are not specific to schizophrenia (e.g., in verbal memory deficits, thought disorder, WCST [Wisconsin Card Sorting Test] performance, etc.) (Clark et al. 2001), and that characteristics found among schizophrenia patients are sometimes not found among high scorers (Silverstein et al. 1992). We believe that any approach that focuses solely on behavior or symptoms is bound to produce little progress, in contrast to approaches that can model and integrate phenomena at multiple levels of analysis.

The theory we propose is consistent with the work of Williams as well as the modeling work of Wright, Edelman, Grossberg and others, in indicating that the nature of the hypothesized integrative deficit is amenable to exploration, and, as noted, above, can be reconciled with developmental models of schizophrenia. We agree with Gooding \& Braun that it would be useful for researchers working within the psychometric high-risk approach to examine NMDA receptor functioning, and related phenomena, among high scorers on the schizotypy scales. Indeed, reduced cognitive coordination, or, as described earlier by Conrad (1958), a neurophysiological abnormality in "differential and integral Gestalt functions" (p. 161) is a candidate mechanism for Meehl's integrative neural defect (schizotaxia). Incorporating these "schizotaxia-close" indicators into subject selection procedures in schizotypy research may prove useful. This sort of focused, theory-driven approach could help increase the yield from schizotypy research by beginning to test some of the core assumptions of Meehl's theory of schizotaxia.

It is important to add here that even within a neurodevelopmental view of schizophrenia, non-genetic factors can be important. A number of studies indicate that altered developmental environments can affect expression of NMDA receptors and increase risk for schizophrenia. Liu et al. (2000) demonstrated that positive maternal care in rats is related to increased expression of genes encoding NMDA receptor subunits, which enhances hippocampal sensitivity to glutamate as well as hippocampal development. This suggests that lower than normal maternal or environmental care may lead to reduced NMDA receptor expression, and possibly, in humans, to an increased risk for schizophrenia. In humans, physical and sexual abuse in childhood has been linked to an increased risk of schizophrenia (Read et al. 2001). While there is not enough evidence at this point to determine whether adverse environmental experiences during development are associated with the development of impaired NMDA receptor expression and cognitive coordination in humans, we suggest that this may be as fruitful an area to explore as that of the behavior genetics of cognitive coordination in schizophrenia.

\section{R6.2. Course of illness after first psychotic episode}

Consistent with findings that cognitive coordination is more related to chronic than acute $\mathrm{PCP} /$ ketamine exposure, two studies indicate that forms of cognitive coordination in schizophrenia are impaired in chronic, but not first-episode patients. Salisbury et al. (2002) found this to be true for MMN, while Parnas et al. (2001) found this to be true for three visual binding tasks. In contrast, other studies have found coordination deficits in unaffected relatives (e.g., MacDonald et al. 2003), and clinical disorganization can be detected in high-risk individuals who develop schizophrenia within a year (Yung et al. 2003). Other studies have found coordination deficits to covary with disorganization among chronic patients (Knight \& Silverstein 1998; Silverstein et al. 1998a; 2000), and to covary with inpatient versus outpatient status (Silverstein et al. 1996a). Clarifying the issue of the relationships between onset of specific coordination deficits, onset and course of clinical features, and course of the illness thus remains a major unsolved task.

\section{R7. Is our perspective relevant to understanding the characteristic self-disturbances of schizophrenia?}

Hemsley notes that disturbances in the operation of context have been invoked to explain disruptions in the "sense of self," which has been viewed as a core disturbance in 
schizophrenia. However, Sass \& Uhlhaas claim that our view "neglects important aspects of consciousness" and offer insights from phenomenological philosophers that they believe "may help clarify the subjective dimension" of schizophrenia, and the role of context in consciousness.

Hemsley's discussion of Epstein and James echoes Sass \& Uhlhaas's discussion of Gurwitsch's (1964) concept of "the margin." Each view posits that in schizophrenia, there is an intrusion into conscious awareness of material that normally would not be included, leading to a reduced ability to organize behavior and cognition in terms of a unified motivational state. These ideas are similar to Sullivan's (1956) observation that in schizophrenia there can be an "escape of attention from its ordinary field," and a subsequent loss of control of the contents of consciousness, leading to the emergence into consciousness of material that is normally excluded. Sullivan saw this as a factor in the failure of the self-system in schizophrenia, because such intrusions have the effect of interfering with a sense of a consistent and coherent self from moment to moment. This is similar to Hemsley's claim that a failure to integrate sensory input with memories of similar experiences leads to a disturbed sense of self.

We are encouraged that a convergence of clinical and theoretical work suggests that the concept of reduced cognitive coordination may be useful in explaining self disturbances in schizophrenia. We also agree with Sass \& Uhlhaas's implication, however, that a comprehensive theory of schizophrenia needs to be informed by first-person data, and be able to describe phenomena at the level of subjective experience. In this way, we see a difference between a disturbed sense of self, as accounted for by a lack of integration of sensory input with memory, and a disturbed sense of self as described by a patient actually having the experience. Ultimately, a strong theory of schizophrenia should be able to describe the latter type of experience at the phenomenological level, and account for its underlying mechanisms.

If our theory is to be relevant for explaining disturbances of consciousness and self-experience in schizophrenia, it will have to be refined and extended to demonstrate that the coordinating mechanisms we discuss are relevant for these phenomena. The suggestion that it is relevant is consistent with the work of the Gestalt psychologist Köhler (1947) who noted that the Gestalt laws apply not only to perception, but to phenomena such as thinking and feeling as well. Similarly, Conrad (1958) believed that a disturbance in integrative gestalt functions, especially involving a loss of figure-ground distinctions between internally generated mental events and external input, causes some of the characteristic experiences of schizophrenia, such as hallucinations, thought disorder and thought broadcasting. More recently, Lehar (2002; in press) proposed a quantitative model in which consciousness itself is viewed as a form of mental representation that involves gestalt properties. Moreover, the hypothesis that binding processes involving synchronization are involved in the generation and maintenance of conscious states of awareness has been proposed (Crick 1994; Varela 1995), and has received experimental support (Lutz 2002). The importance of this recent work is that it can account not only for basic perceptual or other cognitive functions, but for the integrated state of consciousness that is the foundation of conscious and selfexperience. Lehar (in press) notes that the biggest problem with most contemporary neurophysiological theories of vision is that they do not go beyond basic feature recognition to explain the subjective sense of visual consciousness. He also notes that this is a factor in the relative inattention that has been given to gestalt phenomena, which requires theories to go beyond neurophysiological activity in order to account for the subjective experiences described in the gestalt "laws." The recent modeling work of Lehar, Grossberg, Edelman, and others suggests that it is possible to bridge the gap between underlying mechanisms and subjective experience. Therefore, extensions of our theory of cognitive coordination into the realm of consciousness and its disturbances should be possible, and this is exciting territory to explore.

The relevance of disturbed coordinating processes for disturbances at the level of the self is supported by evidence indicating reduced autonoetic awareness in schizophrenia. In two recent studies of nonverbal (Danion et al. 1999) and verbal (Tendolkar et al. 2002) memory, it was demonstrated that schizophrenia patients' disturbances in memories for events could be explained as a function of a reduced ability to integrate recall of events with a subjective sense of having experienced those events. Among patients, and in contrast to controls, recall was based more on a sense of knowing that something occurred, rather than on being able to generate a recollection that included a sense of self-awareness of having experienced the event. ${ }^{6}$ In commenting on the Danion et al. paper, Tien (1999) noted that "events are located in perceptual space and time, and perhaps a basic space-time mental framework is needed to index and organize perceptions as occurring within this framework" (p. 637). Tien also suggested that an internal time code may be needed to form associations. This comment suggests that the sort of timing mechanism that Condray \& Steinhauer noted is involved in language processing may also be relevant for binding together elements of consciousness as a whole.

Sass \& Uhlhaas cite the work of Gurwitsch (1964) as an example of how insights from phenomenology can be applied to schizophrenia. His concepts of the theme, thematic field, and margin are indeed useful, as they extend notions about context and coordination into the realm of everyday behavior, social cognition, and scene perception. These are areas where the concept of cognitive coordination has not yet been explored.

Sass \& Uhlhaas note that, in schizophrenia, there is seldom a breakdown of spatial form (the theme), which they interpret as indicating that it is coordination of the theme with the thematic field that is impaired in schizophrenia. According to Sass \& Uhlhaas, this "loosening of the perceptual context" from the theme (or main focus of awareness) impairs the appreciation of the relevance of material in focal awareness. Stated differently, objects and events in the environment may come to be experienced as if not connected to the intentions, goals, motivations, and affects of the person doing the experiencing. Data suggesting impairments in the processing of basic gestalt information do exist, however (e.g., Cox \& Leventhal 1978; Place \& Gilmore 1980; Silverstein et al. 1996a; 1996b; 1998a; 2000). ${ }^{7}$ Therefore, we suggest, using Gurwitsch's terminology, that disorders of cognitive coordination occur within the theme, as well as between the theme and thematic field. However, we also note, in agreement with Sass \& Uhlhaas, that Gurwitsch's concept of relevancy relationships (characteristic of 
Response/Silverstein \& Phillips: Convergence of perspectives on cognitive coordination in schizophrenia

the thematic field), is useful for understanding schizophrenia. Phenomenological reports from patients (e.g., Sass 1992) certainly do suggest that the normal contextual relationships between objects in the environment, and between a person's ongoing concerns and the environment, are reduced in many people with schizophrenia. To date, while much attention has been paid to integrative functions in perception, the topic of connections between aspects of consciousness has been addressed primarily by phenomenological philosophers. If concepts such as relevancy relationships are to be addressed from a neuroscientific perspective, then scientists will have to begin to address issues such as meaning, intentions, and so on, in their theories of cognition and consciousness. We view this as an important direction, and see Sass \& Uhlhaas's focus on disorders of coordination between the theme and thematic field as deserving of further study. It points the way to a psychology of schizophrenia that views cognitive impairments in this illness as more than merely an isolated dysfunctional circuit or module. Indeed, their commentary highlights the importance of viewing patients' cognitive distortions within the broader context of their experience of the world.

An important issue arising from Sass \& Uhlhaas's commentary involves the methodological approach that would be used to determine whether disorders of consciousness fit within our view of cognitive coordination. While it would certainly be simple enough to determine whether self-fragmentation experiences and/or self-environment dissociation experiences are correlated with biological or laboratory cognitive evidence of impaired coordination, Lutz (2002) has noted that such demonstrations cannot satisfactorily address the real issue, because this approach has as an implicit premise the separation of phenomenal description and scientific explanation. Only third person data are collected, and this approach does not address the phenomenological claim that experience can be manifested to, or affected by, itself.

Stated differently, Lutz sees such designs as unable to account for the emergence of particular phenomenal states and neurobiological signatures in response to external events. To do this successfully, he argues that the study design must be mutually constrained by both phenomenological and biological data. As an example of the latter approach, which he calls generative passages, subjects trained in reporting phenomenal experiences, using the bracketing method of Husserl, described their state of consciousness (e.g., awareness, readiness) prior to each trial in a task in which random dot stereograms were fused, leading to the emergence of a 3-D image of a Necker cube (subject descriptions were given after the trial finished). Subject descriptions naturally grouped into three categories, which were called steady readiness, fragmented readiness, and unreadiness. ERP data from each trial were analyzed as a function of these first-person description categories. In this way, the dynamical neural signature was studied as a function of the phenomenological reports generated by subjects. The results supported the idea that conscious states of readiness, or feelings of continuity with the pre-trial moment, were associated with early, pre-target-onset synchronization within the gamma band. States of unreadiness were characterized by no pre-target gamma synchrony, and a late onset of synchronization, which was interpreted as reflecting an initial breakdown of the task-irrelevant state (accompanied by feelings of discontinuity with the former moment) and then a late onset re-attunement to the task at hand. The implication of Lutz's epistemological argument and data for our theory is that, in order to approach the type of phenomenologically-informed theorizing about schizophrenia suggested by Sass \& Uhlhaas, it will be necessary to generate experimental designs that incorporate the conscious experience of the subject into the study's independent variables. This is an exciting challenge, but one which any strong theory of schizophrenia needs to be able to meet.

\section{R8. Cognitive coordination and creativity}

Both Hoffman \& McGlashan and Park et al. note that factors affecting cognitive coordination and creativity may be related. Hoffman \& McGlashan note that creativity may occur at high levels of synaptic pruning, just before the psychotogenic threshold is crossed. Park et al. note that a disorder of cognitive coordination would allow for mental contents to be experienced outside of their normal context, allowing for novel groupings and relationships. If it is true that reduced connectivity and/or widespread reductions in cognitive coordination are specific to schizophrenia, and if the above hypotheses on creativity are valid, then creativity should be associated with schizophrenia, and this should be a specific form of creativity that is distinct from what is found to be associated with other conditions such as depression and bipolar disorder. This is because existing evidence suggests that among psychiatric disorders, reduced cognitive coordination is relatively specific to schizophrenia. Two sources of evidence suggest that this may be the case. First, past studies have identified differences in thought disorder among schizophrenic and bipolar disorder patients, with the former group demonstrating more bizarre, disorganized speech and the latter demonstrating more playful, combinatory oddities (Solovay et al. 1987). This suggests that when thinking is disordered in these different conditions, it may result from different mechanisms. Second, Sass (1992) has cogently argued that the forms of selffragmentation characteristic of schizophrenia give rise to a particular aesthetic. He cites a wealth of first-person examples of well-known writers, poets, painters, and schizophrenia patients (some of whom are also artists/writers/poets) demonstrating similarities between the phenomenological experiences of schizoid and schizophrenic individuals and aspects of modernist art. We believe that the connection between reduced cognitive coordination and creativity is worth exploring. As noted by Park et al., despite years of hypothesizing about creativity and psychopathology, the research evidence on this issue is mixed, and the positive evidence paints a complex picture involving contributions of psychopathology, childhood experience, and many other factors. While it is unlikely that factors related to schizophrenia are major determinants of the creativity of most artists, it is nevertheless possible, as hypothesized by Sass \& Uhlhaas, that impaired context processing may be common both to schizophrenia spectrum disorders and certain forms of creativity.

\section{R9. Is NMDA activity impaired in disorganized patients?}

No commentary explicitly argued that NMDA dysfunction is not involved, and many explicitly agreed that it is. Several 
asked for further evidence, however, The most important new evidence reported in the commentaries is Javitt's finding that glycine, an NMDA agonist, reduces patient's characteristic errors in the AX-CPT task. This task assesses coordinating interactions, such as those involved in executive control and WM. This complements findings showing that ketamine, an NMDA antagonist, increases healthy volunteer's errors in this task (Umbricht et al 2000).

Oades, Röpcke \& Oknina (hereafter Oades et al.) cite findings of Kapur and Seeman (2002) that are interpreted as evidence that PCP and ketamine have an affinity for D2 and 5HT-2 receptors that is similar to that for NMDA receptors. We have three responses to this. (1) The most important is that Kapur and Seeman's estimate of affinity for NMDA receptors is inadequate, for instance, it was made in the absence of both NMDA and glycine (Javitt, personal communication). ${ }^{8}$ Thus, if their measures of affinity for D2 and 5HT-2 are correct, the findings of Kapur and Seeman do not weaken the NMDA hypothesis, but rather strengthen it, because these findings indicate binding to D2 and 5HT2 receptors similar to Kapur and Seeman's measure of binding to NMDA receptors, which was an underestimate. (2) Dopaminergic and seretonergic systems cannot themselves directly coordinate cognitive activities. Our primary grounds for supposing that NMDA receptors have a coordinating function is that information specifying what we are perceiving, thinking, and doing must be conveyed by the glutamatergic system. What needs to be done is to coordinate these activities with each other. No other system has anywhere near the connectional specificity required to convey the necessary information. On these grounds we expected the glutamatergic system to have both driving and coordinating components. The physiological properties of AMPA and NMDA receptors provide strong support for this prediction. (3) Glycine directly affects NMDA receptors, but not D2 or 5HT-2 receptors. Its therapeutic effects (Javitt) can therefore be more directly explained via the former than the latter.

Oades et al. also ask whether NMDA hypofunction produces too much or too little glutamatergic activity. This is a complex issue requiring further study, but the NMDA hypofunction hypothesis is not a hypoglutamatergic hypothesis as some have claimed (e.g., Kapur \& Seeman 2002). It is compatible with an increase, a decrease, or even with no overall change at all in glutamatergic activity. This is because the hypothesis focuses on the balance between modulation via NMDA receptors and driving activity via non-NMDA receptors, not on the sum total of glutamatergic activity.

Alpert \& Angrist suggest that NMDA dysfunction may be a risk factor that is observable prior to acute onset. We agree, and have data from several studies showing that configural face perception and context sensitivity in size perception are both impaired in that subset of the general population who rate themselves as disorganized on the Schizotypal Personality Questionnaire (e.g., Uhlhaas et al., submitted). This is strong evidence because reduced context-sensitivity in the size perception task (based on the Ebbinghaus illusion) produces better performance in conditions where context is misleading. ${ }^{9}$

Talamini et al. note that NMDA receptors have long been associated primarily with learning, and ask how that fits with our story. As noted in section 3 of the target article, it fits well. We assume that learning is facilitated by coherence and synchronization. Patterns of activity that are internally coherent are learned better than those that are not. The memory deficits outlined in section 4.3. of the target article support the prediction that NMDA hypofunction will impair learning. Hoffman \& McGlashan emphasize this aspect of NMDA function when citing evidence that NMDA receptors may play an important role in maintaining and/or eliminating synaptic connections. We agree that this is well worth further study, but emphasize again that from our perspective effects on learning are a consequence of effects on processing, not independent of them.

Interactions between glutamatergic, dopaminergic, and serotonergic systems are complex. Furthermore, we assume that, in addition to the short-term interactive consequences of a primary malfunction in any one of them, there will be various long-term interactive adaptations. We agree with several commentaries suggesting that there is much undiscovered territory here.

The most unexpected commentary was that by Sanders, Platek \& Gallup (Sanders et al.) suggesting that compensatory adaptation of NMDA functions, for example, in anterior cingulate cortex (ACC), may help explain why blind schizophrenia patients are so rare. This is an intriguing and important idea. We strongly agree with their suggestion that dynamic adaptation of NMDA receptor channels and their sub-unit composition may be of central importance to an understanding of cognitive coordination and its dysfunctions in general.

\section{R10. EEG and high-frequency rhythms}

Several commentaries discuss the relevance of EEG and high-frequency rhythms. Tagamets \& Horwitz see the synchrony of fast rhythms as our main hypothesis. It is not, and no other commentary said that it is. Section 5 , where this is discussed, was added to the target article only after feedback from referees. As Phillips and Singer (1997a) note, it is obviously the case that synchrony does not require periodicity. When the signals synchronized are periodic, however, as they will often be, then synchronized oscillations result.

Titone \& Debruille outline evidence for the relevance of cognitive coordination to language processing and associated ERPs. They note that two potentials thought to reflect contextual interactions, N400 and P600, are impaired in schizophrenia, and particularly in disorganized and nonhallucinating patients. This fits well with our perspective. However, they also note that, although ketamine reduces $\mathrm{N} 400$, it has not yet been shown to reduce P600. We agree that this is well worth further study, but do not see it as greatly weakening our theory. First, a failure to find an effect is not strong evidence. Second, the relation between P600 and context processing is not yet clearly known. Third, acute effects of ketamine are less schizomimetic than chronic ketamine psychosis. Condray \& Steinhauer also cite evidence that processes such as lexical access and semantic memory function are associated with high-frequency rhythms, and argue that timing relations in the processing of rapid sequential stimuli may also be important. Both commentaries suggest that relations between contextsensitive language processing, EEG data, and psychosis are worthy of much further study. We agree.

Crucial issues concerning the relevant analyses to use when interpreting EEG data are raised by Pflieger and by 
Strelets. Pflieger reports that visual patterns presented simultaneously to left and right hemispheres, and differing only in the perceptibility of groupings within those patterns, produce ERPs that differ in some frequency bands, and particularly in the gamma band. These differences were located over the visual cortex, and occurred rapidly, particularly when the pattern with perceptible structure was in the right visual field. Evoked and induced responses were analyzed separately, and were shown to be differently affected by grouping. Though a predicted association between grouping and induced activity was not found, these results show that there are many ways to analyze such data, and that we do not yet know which are the most appropriate. This is also shown clearly by the results outlined by Strelets. She found that spectral power within the gamma band did not distinguish schizophrenic patients, but that coherence of gamma across different hemispheres and different cortical regions did. This fits well with Bressler's perspective, and again highlights the necessity of using appropriate analyses when interpreting EEG data.

As Williams et al. rightly note, the hypothesis of reduced gamma is only a simple first-pass prediction from our perspective. Emphasis upon phase synchrony within and across regions must also be added, as must the relevance of lower-frequency rhythms, for example, as discussed by Kopell (2000). Furthermore, the distinction between induced and evoked rhythms may also be important. Williams et al. outline evidence that distinctions between schizophrenic syndromes should be taken into account when analysing EEG data. We agree that there is much unexplored territory here. Their finding of disturbed gamma activity, particularly in disorganized patients, greatly encourages such further exploration.

\section{R11. Computational theories and computational models}

Seven commentaries call for our perspective to be developed and tested through modelling (Barch \& Braver, Grossberg, Hoffman \& McGlashan, Raffone et al., Tagamets \& Horwitz, Talamini et al., Williams et al.). This was the most frequent suggestion made, so we must take it seriously. We do. The commentaries vary in the amount claimed for modelling. Talamini et al. argue that cognitive neuroscience "might benefit from models." Grossberg argues that they are "needed." Most is claimed by Tagamets \& Horwitz, who say that models are "necessary." In response, we reiterate our distinction between theories and models, which was clearly understood by Barch \& Braver. What we have offered is primarily a theory, not a model (Kay et al. 1998; Phillips \& Singer 1997a; 1997b; Phillips et al. 1995). We did cite both large-scale (e.g., Sporns et al. 1989) and small-scale models (e.g., Lisman et al. 1998) in support of our theory, however. More are now cited by Raffone et al. and by Williams et al.

\section{R11.1. Computational theory}

Science progresses by discovering abstract simplifications that reveal some underlying order in the endless details of existence. In relation to the study of information processing systems, we find Marr's (1982) concept of "computational theory" useful. ${ }^{10}$ This refers to an understanding of the goals of any computation, and of the strategy or strategies by which they can be met. This is clearly distinguished from the representations and algorithms used to specify procedures by which the strategy might be instantiated, and of the hardware in which they may be implemented. Phillips and Singer (1997a, sect. R7.2) try to make this clear using the example of flight. The goal is to stay up in the air. A strategy by which this may be achieved is to use the aerodynamic lift provided by wings with certain cross-sectional profiles, as formalized in the appropriate equations. They say nothing about the materials from which the wings are made, nor about a host of other details concerning any real wing. The theory can be tested using wings with various cross-sections in wind tunnels. They can be used as models of birds' wings, but they are valid tests of the theory of aerodynamic lift whether any organism flies using this strategy or not. Understanding the strategy transforms our understanding of biological flight, however. It tells us what to measure to see whether any biological wing is capable of generating lift in this way. This shows that birds' wings can and insects' wings cannot. The aerodynamic theory can therefore help us understand biological flight without requiring us to build models of either bird or insect wings.

The role of computational theory in the study of brain function is much the same, except for the very important difference that there the goals are far from obvious. (We assume that the computational goals of local cortical circuits are very different from the goals of the organism as a whole.) The concept of "cognitive coordination" begins with the hypothesis that coordinating diverse activities is a general and fundamental goal. This is formalized using information theory. A strategy by which this may be achieved is by the combined use of driving and modulatory connections, together with a learning algorithm for adapting the strengths of these connections based on predictive relationships between stimuli and events. This strategy has been tested by studying the capabilities and limitations of algorithms that instantiate the theory (e.g., Kay et al. 1998; Phillips et al. 1995). Given some understanding of these goals and strategies we can ask whether the theory is of relevance to cortical function. NMDA-receptors and disorganization in psychosis supply evidence that it is.

Barendregt suggests that the $\mathrm{Pi}(\boldsymbol{\pi})$ calculus, developed for the specification and analysis of concurrent communicating processors within computing science may be of use in formalizing the basic concepts of our approach. This is because "communication" in that theory is closely analogous to "coordination" in ours. We very much agree, and are surprised to find that so relevant a formalism has not yet been used by those working on either brain function or artificial neural nets.

\section{R11.2. Models}

There is much disagreement about use of the term "model" (see Webb 2001). One reason for this may be that it is much over-used. There seems to be general agreement that it involves two things, however: the model and whatever is modelled. To build or select a good model, the modeller must know enough about the thing modelled to build or choose something similar. It seems to us that this does not apply to the scientific enterprise in general. When searching for some underlying order we are trying to discover 
something that we do not have, not model something that we do have. Furthermore, the theoretical understanding that we seek is very different from the data to which it relates. Theories try to be simple, general, and final. The data are complex, particular, and endless. ${ }^{11}$

Models can help move us towards a theoretical understanding, however (Webb 2001). Building things, whether physically or as computer simulations of physical things, requires the specification of things that theories can ignore. This tests the relevance of the theory to physically real things. More fundamentally, both Webb and Barch \& Braver argue that it can force us to refine, elaborate, or add to the concepts and hypotheses on which the theory is based. We agree with them. Models that may be relevant to the current concerns are discussed in the following two subsections.

R11.2.1. Models that include analogies to NMDA-receptors. In support of our case, we cited a large-scale model that includes NMDA-receptors (Sporns et al. 1989). This group has since reported several even larger simulations, all supporting the hypotheses we advocate. Williams et al. now cite a large-scale simulation that is being used to study the effects of NMDA-receptors on the emergence of highfrequency cortical EEG rhythms, amongst other things. Smaller and more functionally focussed simulations were also cited in the target article (e.g., Lisman et al. 1998). Raffone et al. now cite others. We agree that their work strengthens the approach we advocate.

R11.2.2. Models that do not yet include analogies to NMDA-receptors. Though modulatory receptors are common throughout the cortex, they are rare in connectionist models of cognition, which usually rely on excitation and inhibition alone. Furthermore, in keeping with our perspective, these models are also rarely concerned with the coordination of diverse activities. The simulations cited by Grossberg, Hoffman \& McGlashan, and by Talamini et al. do not yet include connections analogous to NMDAreceptors. All note that their models could be used to study the effects of such connections, however. We hope that they are. In response to Grossberg, we must note that the evidence shows that NMDA-receptors play a role in processing, not just learning. In response to Hoffman \& McGlashan we must note that although we agree that NMDAhypofunction is likely to affect pruning, we do not see how this could explain the immediate schizomimetic effects of NMDA-antagonists.

If any simulations not including analogs of modulatory connections are shown to be capable of producing adequately coherent percepts, thoughts, and actions, then that will weaken our perspective. It would not disprove the theory, however, nor explain the ubiquity of NMDA-receptors and the effects of NMDA-antagonists. If extending existing models to include analogies to NMDA-receptors can be shown to aid coherence, then that would strengthen the theory, but would of course not prove it.

R11.2.3. Are large-scale biologically realistic models necessary? We have argued that models can help us develop theories. But are they more than that? Are they necessary? Tagamets \& Horwitz claim that large-scale biologically realistic models are necessary to an understanding of the neural substrates of cognition. We agree that large- scale models can often show properties that are unlikely to be discovered in any other way. This does not imply that theories can only be related to the evidence via modelling, however. A comprehensive discussion of the role of models is provided by Webb (2001) and commentators. Overall, they make a strong case for using models. None of them claimed that models are necessary, however, and several explicitly argued that they are not. Biorobotic models have been useful to biology (Webb 2001), but so far not much (Niebur 2001), and engineering has gained more from biology than biology from engineering (Selverston 2001). The discovery of the structure of DNA and its relevance to genetics is an oft-cited example of the usefulness of models to biology. By itself the structure built by Watson from tin and cardboard was far from adequate, however. It certainly did not provide something "from which a compelling deduction of cause and effect can be derived" as requested by Grossberg. Watson's model was of use, nevertheless, and this example shows that useful models need not be large-scale and detailed. On the contrary, his was small and crude. Simple models may be especially useful in the early stages of trying to understand some complex system. Overly detailed models can be as incomprehensible as the system itself (Churchland et al. 1990; Giere 2001; Reeke 2001). In any case, a detailed working model of the cognitive functions impaired in schizophrenia would have to be capable of generating coherent percepts, thoughts, and actions, and of distinguishing fantasy from reality. No such system is likely to be built in the near future.

Thus, though we think that models can be useful, we do not agree that they are necessary. Furthermore, as largescale models can confuse, rather than enlighten, it is important to ask whether they help make the complex comprehensible. If the models advocated by Tagamets \& Horwitz do so then they will make a valuable contribution. If they throw light on cognitive coordination, NMDA-receptors, or cognitive disorganization in psychoses, then they will be of relevance to the issues being discussed here.

\section{R12. Treatment implications of our perspective}

\section{R12.1. Implications for pharmacological interventions}

New data cited by Javitt, indicating that glycine reverses impairments on the MMN and AX-CPT paradigms suggests that agents that enhance NMDA receptor activity can improve cognitive coordination. It remains to be seen whether pharmacologic interventions at glutamate receptors can improve multiple manifestations of reduced cognitive coordination, and whether these improvements covary. The finding that glycine also improves scores on the cognitive factor of the PANSS (which includes conceptual disorganization) suggests that they may (Heresco-Levy et al. 1999). Because glycine also improves negative symptoms, however, this raises the issue of why levels of disorganized and negative symptoms should covary, and whether our theory can account for this, as noted by Ginsberg. Williams et al. suggest reasons why this might be so, and significant zero-order or time-lagged correlations between reductions in disorganized symptoms (or other manifestations of reduced coordination) and reductions in negative symptoms would provide a strong test of their theory.

Goff and Coyle (2001) discussed several pharmacologic intervention strategies to improve NMDA receptor func- 
tioning, including the use of co-agonists such as glycine, partial agonists such as the antitubercular drug D-cycloserine, and ampakines such as CX516 that potentiate AMPA receptor-induced polarization, thereby enhancing NMDA receptor functioning. To date, only glycine has been studied in terms of improving cognitive coordination, and this is an important area for future study.

\section{R12.2. Implications for psychological interventions}

The use of psychological techniques to improve cognitive coordination in schizophrenia provides much opportunity for exploration. Park et al. demonstrated in several studies that altering stimulus and/or task contexts can modify performance on cognitive tasks where performance deficits are considered characteristic of schizophrenia. Our group has demonstrated similar effects (Silverstein et al. 1996a). Such data support the idea that cognitive task performance (or real-world behavior) should not be thought of only in modular fashion. A fuller understanding of the performance strengths and weaknesses in schizophrenia is likely to include an understanding of social, affective, selfrepresentation, and cognitive factors during ongoing behavior.

In general, improved performance among schizophrenia patients on tests thought to tap "hard-wired" deficits has been observed following two types of interventions: (1) the use of monetary incentives; and (2) the use of specific instructions or task manipulations designed to highlight relevant material or reduce complexity (Silverstein et al. 2001; 1996a). Translated into the language of Gurwitsch (1964), discussed by Sass \& Uhlhaas, this suggests that theme-thematic field connections can be strengthened by increasing motivation to perform the task, or by eliminating the presence of information that is likely to interfere with focus on the theme.

Park et al.'s modification of spatial working memory performance in schizophrenia through the use of social manipulations raises questions about what mechanism is involved. Specifically, is improvement due to increased levels of motivation secondary to the social interaction; to improvements in self-esteem secondary to the interaction that subsequently enhance motivation; to a reduction in selffragmentation or self-disturbance provided by the positive interaction, leading to an enhanced readiness state during subsequent cognitive task performance, and/or to other factors? All of these suggestions involve the question of whether addressing the concerns or projects (borrowing from Sass \& Uhlhaas) of the person leads to improved cognitive functioning. Preliminary evidence suggests that this is the case in general, including in cases of cognitive coordination. However, many questions remain about how this works.

These principles may be operative in the use of token economies with so-called "treatment-refractory" schizophrenia patients. The simplification of the environment, the increased feedback about positive and negative consequences of behavior, and the linkage of appropriate behaviors to positive social interactions and secondary reinforcers has led to dramatic improvements in the functioning of patients who did not respond to any other form of treatment (Paul \& Lentz 1977; Silverstein et al. 2002). One way of conceptualizing this is to say that a token economy, through its use of positive (motivating) reinforcers and enhanced stim- ulus salience, improves context processing in schizophrenia patients, allowing for behavior to become more adaptive.

The implications of this idea for cognitive rehabilitation of schizophrenia is that, assuming the presence of relevant treatment content, only treatments that address motivation are likely to succeed. Cognitive rehabilitation approaches that directly aim to promote task engagement through the use of intrinsic (Medalia et al. 1998) or extrinsic (Silverstein et al. 2001) reinforcers appear to be the only forms of cognitive rehabilitation for schizophrenia that demonstrate clinically meaningful real-world improvements in neuropsychological functioning. ${ }^{12}$ "Disembodied" approaches, that conceptualize the work as an attempt to modify an aspect of a computational system, often fail to show any benefit (Benedict et al. 1994). We suggest that in developing new cognitive and psychosocial rehabilitative treatments for schizophrenia, clinicians build in motivational and other coordination-enhancing features. We are currently testing several methods for enhancing awareness of response readiness (as defined by Lutz 2002), to see if these methods improve performance on laboratory (perceptual) measures of cognitive coordination. Recent research indicating that schizophrenia patients can self-regulate their own EEG activity (Gruzelier 2000) also suggests that neurophysiological phenomena such as gamma band synchrony may also be amenable to psychological influence. In developing interventions to enhance cognitive coordination, it will be important to determine which patients benefit from them, and what can be done for patients who do not benefit (e.g., development of assistive devices to improve binding; Tien 1999). Ultimately, it will be important to determine if any techniques that can be used to improve functioning in the laboratory also assist patients in their ability to organize their own experience and behavior in the real world.

\section{ACKNOWLEDGMENTS}

We thank Dan Javitt, Barbara Webb, Nancy Kopell, Adam Savitz, Peter Uhlhaas, Lindsay Schenkel, and Louis Sass for their helpful insights and discussions of unpublished data and manuscripts during the preparation of this response.

\section{NOTES}

1. Ambiguity and its resolution through context is central to language (e.g., Altmann 1998), as is the perception and production of syntactic structure, which involves dynamic grouping. Our concept of dynamic grouping is much the same as Fodor and Pylyshyn's (1988) notion of "compositionality," which applies primarily to higher functions such as language and working memory. Recent evidence also shows that higher perceptual functions, such as the perception of causality, are highly context sensitive. Executive functions may be so commonly impaired in schizophrenia, as emphasised by Cohen and Servan-Schreiber (1992), because they are highly dependent on context-sensitivity and dynamic organisation.

2. Notions of "top-down" and "bottom-up" need to be improved, however. Contextual modulation of activity in primary visual cortex by texture segregating processes within higher visual cortex is both "top-down" and stimulus driven (Zipser et al 1996). As this is contradictory, given normal usage, the terminology should either be improved or abandoned. In the meantime, when we mean "task-based effects" we will say just that.

3. This view is consistent with the "force-field" theory of Aksentijeviç et al. (2001). They proposed a view of perceptual space based in analytical geometry, and on Köhler's (1929) "minimum principle" (or law of prägnanz), in which visual grouping (across space) and auditory grouping (across time and involving memory 
processes) are seen as reflecting a common mechanism. Aksentijeviç et al. (2001) argue that the spatio-temporal equivalence of visual and auditory grouping, as an example of "supramodal invariants of organization" can provide clues to the neural correlates of perception, and to the dependence of perceptual events on their contexts.

4. A recent model in support of the widespread importance of oscillatory mechanisms is the OSCAR (OSCillator-based Associative Recall) model of Brown et al. (2000). The OSCAR model, which is based on both computational modeling and real-world data, demonstrates that the internal representation of sequential information involves the formation of links between internal cognitive states and events in the external world. In this model, internal cortical representations become bound to each other by synchronized oscillations at various time scales. Internal and external events are bound by the association of external events with an endogenous, dynamic learning context signal/vector, which represents the changing state of an array of several endogenous oscillators (operating over a range of fast, medium, and slow frequencies), with each modeled as a signal that varies sinusoidally over time. The changing output of the oscillators causes the internal learning context to change over time, but the predictable relationships between the oscillators (like the relationships between the second, minute, and hour hands of a clock) allow for sequential order to be encoded and for facilitation of order information during recall. In this way, successive states of the system can be reproduced from knowledge of an initial state. In the OSCAR model, encoding involves the formation of associations between successive list items and successive internal dynamic states (modeled as the product of the outputs of the oscillators at any given time point). Recall involves reinstatement of the dynamic learning context, which, of course, assumes the ability to generate/recreate oscillatory activity.

Brown et al.'s model is consistent with our thesis, in that they note that oscillatory activity underlies performance in a variety of areas, including visual perception, spelling, variable binding in reasoning, motor control, short term memory, and time estimation. Brown et al. suggest that oscillators can be viewed as adaptively rational mechanisms that allow for representation of the dynamic statistical properties of the external environment in such a way that optimal behavior can result. The OSCAR model provides a nice example of how a timing mechanism can be reconciled within the view we propose (although, whereas we focus on synchronization within the gamma band, they focus on synchronization of oscillations across varying time scales). It is likely, however, that oscillations in other bands are involved in some of the effects we discuss. Spontaneous transitions between gamma and beta oscillations have been recently reported (Olufsen et al. 2003), interactions between oscillations in several frequency bands have been observed during top-down and bottom-up processing interactions, and the frequency bands in which oscillations occur may depend in part on prior experience with the stimuli (Von Stein et al. 2000). Our theory would have predicted the sort of grouping effects in memory that are addressed by the OSCAR model, but the generation of their model specifically from data on sequential processing highlights the importance of a timing mechanism, as suggested by Condray \& Steinhauer. We agree that if in fact various forms of context processing (simultaneous and temporal/ sequential) are mediated by the same mechanisms, then a unified theory and model will have to account for both forms. We are encouraged that this issue has been noted by several commentators, and that available data (e.g., those of Condray \& Steinhauer) and recent modeling work can be reconciled with the theory we propose.

5. The serotonergic and glutamatergic systems interact considerably. Many effects of NMDA antagonists involve 5-HT2A receptors, and involve excess activity at non-NMDA glutamatergic receptors (Aghajanian \& Marek 2000). Also, 5-HT2A antagonists can block certain behavioral effects of NMDA antagonists (Carlsson 1995; Yamaguchi et al. 1987). In the PFC, 5-HT2A receptor stimulation increases the release of glutamate and the rate of activity at non-NMDA glutamatergic receptors. NMDA antagonists can also lead to increased glutamate release and activity at nonNMDA receptors (Mathe et al. 1998). The effects of this would be to increase feedforward processing of sensory information while reducing modulatory activity at NMDA receptors.

The first pharmacological models of schizophrenia were based on effects of hallucinogenic drugs. It was thought that these drugs produced their effects through antagonism of 5-HT receptors (Gaddum \& Hammeed 1954; Wooley \& Shaw 1954). More recently, electrophysiological studies have demonstrated that activation of 5-HT2A receptors produces large increases in glutamatergic excitatory postsynaptic potentials in the apical dendritic region of layer $\mathrm{V}$ pyramidal cells through a presynaptic mechanism (Aghajanian \& Marek 1999). The effects of NMDA antagonists such as MK-801 are strongly potentiated by LSD, which acts on 5-HT2A receptors (Carlsson 1995). This is thought to cause a deficiency in thalamic filtering, an increase in sensory information being sent to the cortex, and a breakdown of integrative cortical functions (Aghajanian \& Marek 2000). All of this has led to the suggestion that it would be useful to achieve an integration of the psychedelic hallucinogen and glutamatergic theories of schizophrenia (Aghajanian \& Marek 2000).

An important distinction in this discussion is that between local and global or systemic effects. This is important because while the local circuitry that we propose to be abnormal in schizophrenia may rely primarily on one mechanism, which we propose to be NMDA receptor activity, the systemic effects of NMDA receptor antagonists go beyond the local modulatory circuit and involve interactions with other neurotransmitter systems, including dopamine, serotonin, GABA, and acetylcholine (Javitt \& Zukin 1991; Jentsch \& Roth 1999). Thus, it is not the case that all effects of NMDA receptor antagonists will be analogous to those produced at the local circuit level. For example, MK-801, an NMDA receptor antagonist, has effects on dopaminergic and serotonergic activity, which are also involved in cognition. NMDA receptor antagonists thus can be expected to produce both widespread dysfunctions in modulatory activity (because the local circuitry on which we focus is widely distributed throughout the brain), as well as widespread effects on other neurotransmitter systems that are affected by NMDA receptor activity.

6. In the Danion et al. (1999) study of nonverbal memory, recall was impaired at two levels. At one level, associations between pairings of objects was impaired. This was interpreted as a deficit in relational binding, in the sense that patients were not able to combine all aspects of events into a cohesive, memorable, and distinctive whole. This finding is consistent with our hypothesis on the role of cognitive coordination in memory, on the role of NMDA functioning in memory organization (such as that involved in transitive inference), and on the role of oscillatory activity in grouping and order effects in memory (Brown et al. 2000). At another level, schizophrenic patients' recall disturbances in the Danion et al. study were viewed as a failure to bind event-related information with self-representation information, or, stated differently, a failure to bind self-awareness with information about the external world during ongoing experience.

Studies such as those of Danion et al. and Tendolkar et al. (2002) provide a link between concepts of binding that address neural circuitry at the stimulus feature level, and phenomenological concerns about self cohesiveness and fragmentation, which some consider to be core phenomena in schizophrenia (see Sass \& Uhlhaas commentary).

Such studies also extend past discussions of the directionality of cognitive coordination failures. For example, while basic coordination failures may be responsible for disturbances ranging from gestalt perception to subjective feelings of experiencing the environment as a sequence of single objects without an integrated context, it is unknown at this point how failures in binding self-awareness with ongoing experience may alter the motivational and affective stance toward the environment so as to produce further 
alterations in basic perceptual processes. All of this stresses the need to explore whether our cognitive coordination theory can be extended to account for autonoetic awareness, and the level of binding necessary to achieve a cohesive self-representation and integration of this representation with external events during ongoing experience.

7. In many cases, however, these impairments are only evident under conditions of rapid stimulus exposure. While under normal circumstances patients may not see objects as fragmented, slowness in the processing of gestalts, the formation of noisy representations, or unusually high amounts of competition between perceived objects and other objects and features in the environment for attention, are likely to have serious consequences for the schizophrenia patient (Silverstein et al. 1998a; 2000). These include slowness or interference in accessing lexical information linked to objects, stimulus overload, the capture of attention by irrelevant information, and so on. Moreover, while there are reports of patients seeing objects as fragmented (Arieti 1979; Chapman 1966; McGhie \& Chapman 1961), it appears to be the case that under normal circumstances, these patients can use compensatory strategies to normalize the perception of the object. This is demonstrated in the following examples: (1) "I have to put things together in my head. If I look at my watch I see the watch, watchstrap, face, hands, and so on, then I have got to put them together to get it into one piece" (Chapman 1966); and (2) "Everything is in bits. You put the picture up bit by bit into your head. It's like a photograph that's torn in bits and put together again" (McGhie \& Chapman 1961).

8. Javitt (personal communication) provides more detail on this as follows: "Kapur and Seeman (2002) reported affinities of 2, 5 , and $37 \mu \mathrm{M}$ for PCP binding to the NMDA, 5-HT2 and D2 sites, respectively. Although the 5-HT2 and D2 affinities may be correct, the NMDA affinities are substantially higher than the affinities reported by others, which are generally in the range of 35$250 \mathrm{nM}(0.035-0.25 \mu \mathrm{M})$ (e.g., Nadler et al. 1990; Zukin \& Zukin 1979; Zukin et al. 1983). Further, the apparent affinity of PCP and other noncompetitive antagonists is known to depend upon the ability of these compounds to gain access to the open channel. When assays are conducted in the absence of glutamate and glycine (as in Kapur \& Seeman 2002) long incubation times are required to permit accurate affinity determination. In the Kapur study, the $2 \mathrm{hr}$ incubation time is substantially below the minimum time for the reaction to achieve equilibrium (Javitt \& Zukin 1989). Conducting binding studies under non-equilibrium conditions is well known to lead to a substantial overestimate in affinity, as apparently occurred. Kapur also reported an EC50 of $4 \mu \mathrm{M}$ for interaction of PCP with D2 receptors on CHO neurons. This concentration is 4 time higher than serum and CSF levels associated with lethality during PCP overdose ( Javitt \& Zukin 1991), and unlikely to be of relevance physiologically."

9. A pre-existing cognitive style that is biased toward local processing rather than toward global coordination in no way prevents exacerbation of that bias during acute episodes, however, and may well make it more likely.

10. In relation to the analysis developed by Webb (2001), computational theory may be seen as a source of ideas that can be used to develop a model of some target system.

11. Some say that theories are models. If so, we have a model. We think that this terminology is confusing, however, so do not claim to have a model. Computational instantiations of the Coherent Infomax theory show that the concepts are well specified, but we have not shown that it applies to simulated neural nets that are constrained by relevant measures made on real brains. Indeed, our computational studies suggest that various approximations would be needed if applied to large networks and complex environments. After extensive discussion, Webb (2001) concludes that something is a model only if used as such. According to this view, our theory it is not a model, as we do not use it as one.

12. The discussion here focuses on cognitive rehabilitation of neuropsychological processes, as opposed to cognitive behavioral treatment of symptoms. These two forms of treatment have, to date, had distinct aims. The recent demonstrations of success of cognitive behavioral treatment for symptoms such as hallucinations and delusions is impressive (Dickerson 2000; Pilling et al. 2002). Thus far, however, there have been no studies which have looked at the neuropsychological effects of cognitive behavior therapy for schizophrenia.

\section{References}

Letters " $a$ " and " $r$ " appearing before authors' initials refer to target article and response, respectively.

Abely, P. \& Carton, C. (1967) The man of night and the man of silence: Prologue to a report on a comparative psychopathological and psychiatric study of the blind and deaf. Annals Medico Psychologiques 2:111-25. [GSS]

Abi-Saab, W. M., D’Souza, D. C., Moghaddam, B. \& Krystal, J. H. (1998) The NMDA model for schizophrenia: Promise and pitfalls. Pharmacopsychiatry 31:104-109. [aWAP]

Adler, C. M., Goldberg, T. E., Malhotra, A. K., Pickar, D. \& Breier, A. (1998a) Effects of ketamine on thought disorder, working memory, and semantic memory in healthy volunteers. Biological Psychiatry 43:811-16. [aWAP]

Adler, C. M., Malhotra, A. K., Elman, I., Goldberg, T. E., Egan, M., Pickar, D. \& Breier, A. (1999) Comparison of ketamine-induced thought disorder in healthy volunteers and thought disorder in schizophrenia. American Journal of Psychiatry 156:1646-49. [aWAP]

Adler, L. E., Olincy, A., Waldo, M., Harris, J. G., Griffith, J., Stevens, K., Flach, K., Nagamoto, H., Bickford, P., Leonard, S. \& Freedman, R. (1998b) Schizophrenia, sensory gating, and nicotinic receptors. Schizophrenia Bulletin 24:189-202. [MAK, aWAP]

Adler, L. E., Pachtman, E., Franks, R. D., Pecevich, M., Waldo, M. C. \& Freedman, R. (1982) Neurophysiological evidence for a defect in neuronal mechanisms involved in sensory gating in schizophrenia. Biological Psychiatry 17:639-54. [MAK]

Adler, L. E., Rose, G. \& Freedman, R. (1986) Neurophysiological studies of sensory gating in rats: Effects of amphetamine, phencyclidine, and haloperidol. Biological Psychiatry 21:787-98. [MAK]

Aghajanian, G. K. \& Marek, G. J. (1999) Serotonin 5-HT2A receptors increase ESPCs in layer V pyramidal cells of prefrontal cortex by an asynchronous mode of glutamate release. Brain Research 825:161-71. [rSMS]

(2000) Serotonin model of schizophrenia: Emerging role of glutamate mechanisms. Brain Research Reviews 31:302-12. [rSMS]

Ahveninen, J., Kähkönen, S., Pennanen, S., Liesivuori, J., Ilmoniemi, R. J. \& Jääskiläinen, I. P. (2002) EEG and MEG measurements after tryptophan depletion suggest serotonergic modulation of auditory involuntary attention. Neuroimage 16:1052-61. [RDO]

Ahveninen, J., Kahkonen, S., Tiitinen, H., Pekkonen, E., Huttunen, J., Kaakkola, S., Ilmoniemi, R. J. \& Jaaskelainen, I. P. (2000) Suppression of transient 40$\mathrm{Hz}$ auditory response by haloperidol suggests modulation of human selective atttention by dopamine D2 receptors. Neuroscience Letters 292:29-32. [LMW]

Aksentijeviç, A., Elliott, M. A. \& Barber, P. (2001) Dynamics of perceptual grouping: Similarities in the organization of visual and auditory groups. Visual Cognition 8:349-58. [rSMS]

al-Amin, H. A. \& Schwarzkopf, S. B. (1996) Effects of the PCP analog dizocilpine on sensory gating: Potential relevance to clinical subtypes of schizophrenia. Biological Psychiatry 40:744-54. [MEP]

Alexander, G. E. \& Crutcher, M. D. (1990) Functional architecture of basal ganglia circuits: Neural substrates of parallel processing. Trends in Neuroscience 13:266-71. [aWAP]

Allen, H. A., Liddle, P. F. \& Frith, C. D. (1993) Negative features, retrieval processes and verbal fluency in schizophrenia. British Journal of Psychiatry 163:769-75. [LMW]

Allen, R. M. \& Young, S. J. (1978) Phencyclidine-induced psychosis. American Journal of Psychiatry 135:1081-84. [aWAP]

Allman, J. M., Miezin, F., \& McGuinness, E. (1985) Direction- and velocityspecific responses from beyond the classical receptive field in the middle temporal visual area (MT). Perception 14(2):105-26. [YC]

Alpert, M., ed. (1985) Controversies in schizophrenia: Changes and constancies. Erlbaum. [MA]

Alpert, M., Angrist, B., Diamond, F. \& Gershon, S. (1970) Comparison of ditran intoxication and acute alcohol psychoses. In: Origins and mechanisms of hallucinations, ed. W. Keup. Plenum Press. [MA] 
Alpert, M. \& Bogorad, D. D. (1975) Reduction of sensory sharpening processes associated with chronic alcoholism. In: Alcohol intoxication and withdrawal. Experimental Studies II, ed. M. M. Gross. Advances in experimental medicine and biology, vol. 59. Plenum Press. [MA]

Alpert, M. \& Friedhoff, A. J. (1980) An undopamine hypothesis of schizophrenia. Schizophrenia Bulletin 6:387-90. [MA]

Alpert, M. \& Martz, M. J. (1977) Cognitive views of schizophrenia in light of recent studies of brain asymmetry. In: Psychopathology and brain dysfunction, ed. C. Shagass, S. Gershon \& A. J. Friedhoff. Raven Press. [MA]

Alpert, M., Rosenberg, S. D., Pouget, E. R \& Shaw, R. (2000) Prosody and lexical accuracy in flat affect schizophrenia. Psychiatry Research 97:107-18 [MA]

Alpert, M., Rubinstein, H. \& Kesselman, M. (1976) Asymmetry of information processing in hallucinators and nonhallucinators. Journal of Nervous and Mental Diseases 162(4):258-65. [MA]

Alpert, M. \& Silvers, K. N. (1970) Perceptual characteristics distinguishing auditory hallucinations in schizophrenia and acute alcoholic psychoses. American Journal of Psychiatry 127(3):298-302. [MA]

Altmann, G. T. M. (1998) Ambiguity in sentence processing. Trends in Cognitive Sciences 2:146-52. [rSMS]

American Psychiatric Association (1989) Treatment of psychiatric disorders: A task force report of the American Psychiatric Association, vol. 2. American Psychiatric Association. [aWAP]

Andreasen, N. C. (1999) A unitary model of schizophrenia. Archives of General Psychiatry 56:781-93. [aWAP]

Andreasen, N. C., Arndt, S., Alliger, R., Iller, D. \& Flaum, M. (1995) Symptoms of schizophrenia: Methods, meanings, and mechanisms. Archives of General Psychiatry 52:341-51. [aWAP]

Andreasen, N. C., Paradiso, S. \& O’Leary, D. S. (1998) “Cognitive dysmetria” as an integrative theory of schizophrenia: A dysfunction in cortical-subcorticalcortical circuitry? Schizophrenia Bulletin 24:203-18. [RC]

Andrew, A. (2000) A computational model of information processing in the frontal cortex and basal ganglia. Journal of Cognitive Neuroscience 12:505-19. [LMT]

Andrews S., Shelley, A. M., Ward, P. B., Fox, A., Catts, S. V. \& McConaghy, N. (1993) Event-related potential indices of semantic processing in schizophrenia. Biological Psychiatry 34:443-58. [DT]

Angelopoulos, E. K., Markianos, M., Daskalopoulou, E. G. Hatzimanolis, J. \& Tzemos, J. (2002) Changes in central serotonergic function as a correlate of duration of illness in paranoid schizophrenia. Psychiatry Research 110:9-18. [RDO]

Angrist, B. (1972) Amphetamine psychosis: Clinical variations of the syndrome. In Amphetamine and its Analogs, ed. A. K. Cho \& D. S. Segal. Academic Press 387-414. [MA]

Angrist, B. \& Gershon, S. (1970) The phenomenology of experimentally induced amphetamine psychosis: Preliminary observations. Biological Psychiatry 2:95-107. [MA]

Anscombe, R. (1987) The disorder of consciousness in schizophrenia. Schizophrenia Bulletin 11:241-60. [DRH]

Arieti, S. (1979) Interpretation of schizophrenia, $2^{\text {nd }}$ edition. Basic Books. [rSMS]

Arnsten, A. F. T. (1998) Catecholamine modulation of prefrontal cortical cognitive function. Trends in the Cognitive Sciences 2:436-47. [aWAP]

Ashby, F. G., Isen A. M. \& Turken A. U. (1999) A neuropsychological theory of positive affect and its influence on cognition. Psychological Review 106(3):529-50. [SP]

Astington, J. W., Pelletier, J. \& Homer, B. (2002) Theory of mind and epistemological development: The relation between children's second-order false-belief understanding and their ability to reason about evidence. New Ideas in Psychology 20(2-3):131-44. [DL]

Baddeley, A. D. (1996) Exploring the central executive. Quarterly Journal of Experimental Psychology 49A:5-28. [aWAP]

Bakker, C. B. \& Amini, F. B. (1961) Observations on the psychotomimetic effects of Serynl. Comprehensive Psychiatry 2:269-80. [aWAP]

Baldeweg, T., Spence, S., Hirsch, S. R. \& Gruzelier, J. (1998) Gamma-band electroencephalographic oscillations in a patient with somatic hallucinations. The Lancet 352:620-21. [aWAP]

Balla, A., Hashim, A., Burch, S., Javitt, D. C., Lajtha, A. \& Sershen, H. (2001) Phencyclidine-induced dysregulation of dopamine response to amphetamine in prefrontal cortex and striatum. Neurochemical Research 26:1001-1006. [DCJ]

Barch, D. M. \& Berenbaum, H. (1997) The effect of language production manipulations on negative thought disorder and discourse coherence disturbances in schizophrenia. Psychiatry Research 71:115-27. [aWAP]

Barch, D. M., Carter, C. S., Hachten, P. C., Usher, M. \& Cohen, J. D. (1999) The "benefits" of distractability: Mechanisms underlying increased Stroop effects in Schizophrenia. Schizophrenia Bulletin 25:749-62. [aWAP, rSMS]
Barch, D. M., Carter, C. S., MacDonald, A., Braver, T. S. \& Cohen, J. D. (2003) Context processing deficits in schizophrenia: Diagnostic specificity, four-week course, and relationships to clinical symptoms. Journal of Abnormal Psychology 112:132-43. [DMB]

Baron-Cohen, S. (1995) Mindblindness: An essay on autism and Theory of Mind. MIT Press. [DL]

Baron-Cohen, S. \& Hammer J. (1997) Parents of children with Asperger syndrome: What is the cognitive phenotype? Journal of Cognitive Neuroscience 9:548-54. [aWAP]

Basar, E., Rosen, B., Basar-Eroglu, C. \& Greitschus, F. (1987) The associations between $40 \mathrm{~Hz}-\mathrm{EEG}$ and the middle latency response of the auditory evoked potential. International Journal of Neuroscience 33:103-17. [MAK]

Beauchamp, M. S., Petit, L., Ellmore, T. M., Ingeholm, J. \& Haxby, J. V. (2001) A parametric fMRI study of overt and covert shifts of visuospatial attention. Neuroimage 14:310-21. [RDO]

Beaumont, J. G. \& Dimond, S. J. (1973) Brain disconnection and schizophrenia. British Journal of Psychiatry 123(577):661-62. [YC]

Bender, S., Müller, B., Oades, R. D. \& Sartory, G. (2001) Conditioned blocking and schizophrenia: A replication and study of the role of symptoms, age, onset-age of psychosis and illness-duration. Schizophrenia Research 49: 157-70. [RDO]

Benedict, R. H., Harris, A. E., Markow, T., McCormick, J. A., Nuechterlein, K. H. \& Asarnow, R. F. (1994) Effects of attention training on information processing in schizophrenia. Schizophrenia Bulletin 20:537-46. [rSMS]

Benes, F. M. (2000a) Alterations of neural circuitry within layer II of the anterior cingulate cortex in schizophrenia. Journal of Psychiatric Research 33:511-12. [RDO]

(2000b) Emerging principles of altered neural circuitry in schizophrenia. Brain Research Reviews 31:251-69. [aWAP]

Benes, F. M. \& Coyle, J. T. (1998) Déja-vu all over again. Biological Psychiatry 43:781-82. [aWAP]

Blackwell, S. L., McIntyre, C. W. \& Murray, M. E. (1983) Information processed from brief visual displays by learning disabled boys. Child Development 54:927-40. [aWAP]

Blaney, P. H. (1999) Paranoid conditions. In: Oxford textbook of psychopathology ed. T. Millon, P. H. Blaney \& R. D. Davis. Oxford Unversity Press. [JPG]

Bleuler, E. (1911/1961) Dementia praecox oder die Gruppe der Schizophrenia. In: Handbuch der psychiatrie (Handbook of psychiatry), ed. G. Ascheffenburg. Deuticke. [rSMS] English translation: Dementia Praecox or the Group of Schizophrenias.] International Universities Press. (English edition, 1961). [YC]

Blumenfeld, L. D. \& Clementz, B. A. (2001) Response to the first stimulus determines reduced auditory evoked response suppression in schizophrenia Single trials analysis using MEG. Clinical Neurophysiology 112:1650-59. [rSMS]

Bock, J. \& Braun, K. (1999) Blockade of N-methyl-D-aspartate receptor activation suppresses learning-induced synaptic elimination. Proceedings of the National Academy of Sciences USA 96:2485-90. [REH]

Born, R. T. (2000) Center-surround interactions in the middle temporal visual area of the owl monkey. Journal of Neurophysiology 84(5):2658-69. [YC]
[Y.

Boutros, N. N., Torello, M. W., Barker, B. A., Tueting, P. A., Wu, S. C. \& Nasrallah, H. A. (1995) The P50 evoked potential component and mismatch detection in normal volunteers: Implications for the study of sensory gating. Psychiatry Research 57:83-88. [MEP]

Bowers, K. S., Regher, G., Balthazard, C. \& Parker, K. (1990) Intuition in the context of discovery. Cognitive Psychology 22:72-110. [SP]

Bradski, G., Carpenter, G. A. \& Grossberg, S. (1994) STORE working memory networks for storage and recall of arbitrary temporal sequences. Biological Cybernetics 71:469-80. [SG]

Braff, D. L., Geyer, M. A. \& Swerdlow, N. R. (2001) Human studies of prepulse inhibition of startle: Normal subjects, patient groups, and pharmacological studies. Psychopharmacology (Berlin) 156:234-58. [MAK]

Braff, D. L., Saccuzzo, D. P. \& Geyer, M. A. (1991) Information processing dysfunctions in schizophrenia: Studies of visual backward masking, sensorimotor gating, and habituation. In: Handbook of schizophrenia, vol. 5 Neuropsychology, psychophysiology and information processing, ed. S. R. Steinhauer, J. H. Gruzelier \& J. Zubin. Elsevier Science. [RC]

Braff, D. L., Swerdlow, N. R. \& Geyer, M. A. (1999) Symptom correlates of prepulse inhibition deficits in male schizophrenic patients. American Journal of Psychiatry 156:596-602. [DCG, DRH]

Braitenberg, V. \& Schüz, A. (1991) Anatomy of the cortex. Springer-Verlag. [aWAP]

Braver, T. S., Barch, D. M. \& Cohen, J. D. (1999) Cognition and control in schizophrenia: A computational model of dopamine and prefrontal function. Biological Psychiatry 46:312-28. [DMB, aWAP, LMT]

Breier, A., Malhotra, A. K., Pinals. D. A., Weisenfeld. N. I. \& Pickar, D. (1997) Association of ketamine induced psychosis with frontal activation of the prefrontal cortex in healthy volunteers. American Journal of Psychiatry 154:805-11. [aWAP] 
Breitenseher, M., Uhl, F., Wimberger, D., Deecke, L., Trattnig, S. \& Kramer, J. (1998) Morphological dissociation between visual pathways and cortex: MRI of visually-deprived patients with congenital peripheral blindness. Neuroradiology 40:424-27. [GSS]

Breitmeyer, B. G. \& Ganz, L. (1976) Implications of sustained and transient channels for theories of visual pattern masking, saccadic suppression, and information processing. Psychological Review 83:1-36. [RC]

Bressler, S. L. (1996) Interareal synchronization in the visual cortex. Behavioral Brain Research 76:37-49. [SLB]

(1999) The dynamic manifestation of cognitive structures in the cerebral cortex In: Understanding representation in the cognitive sciences, ed. A. Riegler, M. Peschl \& A. von Stein. Kluwer Academic. [SLB]

(2002) Understanding cognition through large-scale cortical networks. Current Directions in Psychological Science 11:58-61. [SLB]

(2003) Cortical coordination dynamics and the disorganization syndrome in schizophrenia. Neuropsychopharmacology 28:535-539. [SLB]

Bressler, S. L., Coppola, R. \& Nakamura, R. (1993) Episodic multiregional cortical coherence at multiple frequencies during visual task performance. Nature 366:153-56. [aWAP]

Bressler, S. L. \& Kelso, J. A. S. (2001) Cortical coordination dynamics and cognition. Trends in Cognitive Sciences 5:26-36. [SLB, aWAP]

Broadbent, D. E. (1971) Decision and stress. Academic Press. [DRH]

Brown, G. D. A., Preece, T. \& Hulme, C. (2000) Oscillator-based memory for serial order. Psychological Review 107:127-81. [rSMS]

Bundesen, C. (1990) A theory of visual attention. Psychological Review 97:523-47. [AR]

Burgess, A., Strelets, V., Golicova, J., Novototsky-Vlasov, V., Lehman, D. \& Gruzelier, J. (submitted) Neural complexity and integration in unmedicated schizophrenia. International Journal of Psychophysiology. [VS]

Butler, P. D, DeSanti, L. A., Maddox, J., Harkavy-Friedman, J. M., Amador, X. F., Goetz, R. R., Javitt, D. C. \& Gorman, J. M. (2003) Visual backward-masking deficits in schizophrenia: Relationship to visual pathway function and symptomatology. Schizophrenia Research 59:199-209. [RC]

Butler, P. D., Schechter, I., Zemon, V., Schwartz, S. G., Greenstein, V. C., Gordon, J., Schroeder, C. E. \& Javitt, D. C. (2001) Dysfunction of early stage visual processing in schizophrenia. American Journal of Psychiatry 158:1126-33. [DCJ]

Cadenhead, K. S., Geyer, M. A., Butler, R. W., Perry, W., Sprock, J. \& Braff, D. L. (1997) Information processing deficits of schizophrenia patients: Relationship to clinical ratings, gender, and medication status. Schizophrenia Research 28:51-62. [RC]

Cardno, A. G., Pak, C. S., Murray, R. M. \& McGuffin, P. (2001) Twin study of symptom dimensions in psychoses. British Journal of Psychiatry 179:39-45. [aWAP, rSMS]

Carlsson, A. (1995) The selective 5- $\mathrm{HT}_{2 \mathrm{~A}}$ receptor antagonist MDL 100, 907 counteracts the psychomotor stimulation ensuing manipulations with monoaminergic, glutamatergic, or muscarinic transmission in the mouse: Implications for psychosis. Journal of Neural Transmission 100:225-37. [rSMS]

Carr, V. \& Wale, J. (1986) Schizophrenia: An information processing model. Australian and New Zealand Journal of Psychiatry 20:136-55. [aWAP]

Catts, S. V., Shelley, A., Ward, P. B., Liebert, B., McConaghy, N., Andrews, S. \& Michie, P. T. (1995) Brain potential evidence for an auditory sensory memory deficit in schizophrenia. American Journal of Psychiatry 152:213-19. [aWAP]

Chapman, J. (1966) The early symptoms of schizophrenia. British Journal of Psychiatry 112:225-51. [rSMS]

Chapman, L. J., Chapman, J. P. \& Daut, R. L. (1976) Schizophrenic inability to disattend from strong aspects of meaning. Journal of Abnormal Psychology $85: 35-40 . \quad$ [DT]

Chapman, L. J., Chapman, J. P., Kwapil, T. R., Eckblad, M. \& Zinser, M. C. (1994) Putatively psychosis-prone subjects 10 years later. Journal of Abnormal Psychology 103:171-83. [rSMS]

Chen, E. Y. H. \& McKenna, P. J. (1996) Memory dysfunction in schizophrenia. In Schizophrenia: A neuropsychological perspective, ed. C. Pantelis, H. E. Nelson \& T. R. E Barnes. Wiley. [aWAP]

Chen, Y., Nakayama, K., Levy, D. L., Matthysse, S. W. \& Holzman, P. S. (1999a) Psychological isolation of motion processing deficits in schizophrenic patients and their relatives and its relation to eye tracking deficits. Proceeding of National Academy Sciences USA 96:4724-29. [YC]

(2001) Global and local motion processing in schizophrenia. Schizophrenia Research 49:213. [aWAP]

(2003) Processing of global, but not local, motion direction is deficient in schizophrenia processing. Schizophrenia Research 61:215-27. [YC]

Chen, Y., Palafox, G., Nakayama, K., Levy, D. L., Matthysse, S. W. \& Holzman, P. S. (1999b) Motion perception in schizophrenia. Archives of General Psychiatry 56:149-54. [YC]

Chevigny, H. \& Braverman, S. (1950) The adjustment of the blind. Yale University Press. [GSS]
Chey, J., Grossberg, S. \& Mingolla, E. (1997) Neural dynamics of motion grouping: From aperture ambiguity to object speed and direction. Journal of the Optical Society of America 14:2570-94. [SG]

Chey, J. \& Holzman, P. S. (1997) Perceptual organization in schizophrenia: The employment of gestalt principles. Journal of Abnormal Psychology 106:530 38. [YC, aWAP, rSMS]

Churchland, P. S., Koch, C. \& Sejnowski, T. J. (1990) What is computational neuroscience? In: Computational neuroscience, ed. E. L. Schwartz. MIT Press. [rSMS]

Clark, L., Iversen, S. D. \& Goodwin, G. M. (2001) A neuropsychological investigation of prefrontal cortex involvement in acute mania. American Journal of Psychiatry 158:1605-11. [rSMS]

Clementz, B. A., Barber, S. K. \& Dzau, J. R. (2002) Knowledge of stimulus repetition affects the magnitude and spatial distribution of low-frequency event-related brain potentials. Audiology and Neurootology 7:303-14. [rSMS]

Clementz, B. A. \& Blumenfeld, L. D. (2001) Multichannel electroencephalographic assessment of auditory evoked response suppression in schizophrenia. Experimental Brain Research 139:377-90. [MAK, rSMS]

Clementz, B. A., Blumenfeld, L. D. \& Cobb, S. (1997) The gamma band response may account for poor P50 suppression in schizophrenia. NeuroReport 8: 3889-93. [MAK, MEP, aWAP]

Cohen, J. D., Barch, D. M., Carter, C. S. \& Servan-Schreiber, D. (1999a) Contextprocessing deficits in schizophrenia: Converging evidence from three theoretically motivated tasks. Journal of Abnormal Psychology 108:120-33. [DCJ, aWAP]

(1999b) Schizophrenic deficits in the processing of context: Converging evidence from three theoretically motivated cognitive tasks. Journal of Abnormal Psychology 108:120-33. [DMB]

Cohen, J. D., Braver, T. S. \& O’Reilly, R. (1996) A computational approach to prefrontal cortex, cognitive control and schizophrenia: Recent developments and current challenges. Philosophical Transactions of the Royal Society of London 351:1515-27. [DRH]

Cohen, J. D. \& Servan-Schreiber, D. (1992) Context, cortex, and dopamine: A connectionist approach to behavior and biology in schizophrenia. Psychological Review 99:5-77. [DCJ, AWM, SP, aWAP, rSMS, DT]

Condray, R., Steinhauer, S. R., van Kammen, D. P. \& Kasparek, A. (2002) The language system in schizophrenia: Effects of capacity and linguistic structure. Schizophrenia Bulletin 28:475-90. [RC]

Conrad, K. (1958) Die Beginnende Schizophrenie. Versuch einer Gestalt Analyse des Wahns, $3^{\text {rd }}$ edition. G. Thieme. [aWAP, LAS, rSMS]

Contestabile, A. (2000) Roles of NMDA receptor activity and nitric oxide production in brain development. Brain Research Reviews 32:476-509. [REH]

Corcoran, R., Mercer, G. \& Frith, C. D. (1995) Schizophrenia, symptomatology, and social inference: Investigating "theory of mind" in people with schizophrenia. Schizophrenia Research 17:5-13. [rSMS]

Cosgrove, J. \& Newell, T. G. (1991) Recovery of neuropsychological functions during reduction in use of phencyclidine. Journal of Clinical Psychology 47:159-69. [aWAP]

Coull, J. T. (1998) Neural correlates of attention and arousal: Insights from electrophysiology, functional neuroimaging and psychopharmacology. Progress in Neurobiology 55:343-61. [RDO]

Cox, M. D. \& Leventhal, D. N. (1978) A multivariate analysis and modification of a preattentive perceptual dysfunction in schizophrenia. Journal of Nervous and Mental Disease 166:709-18. [aWAP, rSMS]

Coyle, J. T. (1996) The glutamatergic dysfunction hypothesis for schizophrenia. Harvard Review of Psychiatry 3:241-53. [aWAP]

Crick, F. (1994) The astonishing hypothesis. Touchstone. [rSMS]

Cromwell, R. (1975) Assessment of schizophrenia. Annual Review of Psychology 26:593-619. [aWAP]

Csibra, G., Davis, G., Spratling, M. W. \& Johnson, M. H. (2000) Gamma oscillations and object processing in the infant brain. Science 290:582-85. [aWAP]

Curtis, C., Calkins, M. E., Grove, W. M., Feil, K. J. \& Iacono, W. G. (2001) Saccadic disinhibition in patents with acute and remitted schizophrenia and their first-degree biological relatives. American Journal of Psychiatry 158:100-106. [aWAP]

Cutting, J. (1989) Gestalt theory and psychiatry: Discussion paper. Journal of the Royal Society of Medicine 82:429-32. [aWAP]

Cutting, J. \& Dunne, F. (1989) Subjective experience of schizophrenia. Schizophrenia Bulletin 15:217-31. [aWAP]

Damasio, A. R. (1994) Descartes' error: Emotion, reason and the human brain. Putnam. [aWAP]

Danion, J.-M., Rizzo, L. \& Bryant, A. (1999) Functional mechanisms underlying recognition memory and conscious awareness in patients with schizophrenia. Archives of General Psychiatry 56:639-44. [rSMS]

Das, A. \& Gilbert, C. D. (1995) Long-range horizontal connections and their role in cortical reorganization revealed by optical recording of cat primary visual cortex. Nature 375:780-84. [rSMS] 
Davis, K. L., Kahn, R. S., Ko, G. \& Davidson, M. (1991) Dopamine in schizophrenia: A review and reconceptualization. American Journal of Psychiatry 148:1474-86. [aWAP]

Daw, N. W., Stein, P. S. G. \& Fox, K. (1993) The role of NMDA receptors in information processing. Annual Review of Neuroscience 16:207-22. [aWAP]

DeBelleroche, J., Humphries, C., Durnin, A., Mortimer A. \& Hirsch, S. (1998) Regional specificity of changes in the expression of NMDA receptor and cholecystokinin mRNA in schizophrenia. Schizophrenia Research 29:96. [GSS]

DeBeni, R. \& Cornoldi, C. (1988) Imagery limitations in totally congenitally blind subjects. Journal of Experimental Psychology: Learning, Memory, and Cognition 14:650-55. [GSS]

Debruille, J. B. (1998) N400 and knowledge inhibition: Tests with words that look like common words. Brain and Language 62:202-20. [DT]

Debruille, J. B., Pineda, J. \& Renault, B. (1996) N400-like potentials elicited by faces and knowledge inhibition. Brain Research: Cognitive 4:133-44. [DT]

Decker, R. \& Koole, F. D. (1992) Visually impaired children's visual characteristics and intelligence. Developmental Medicine and Child Neurology 34:123-33. [GSS]

Deiber, M. P., Ibanez, V., Honda, M., Sadato, N., Raman, R. \& Hallett, M. (1995) Cerebral processes related to visuomotor imagery and generation of simple finger movements studied with positron emission tomography. NeuroImage 7:73-85. [GSS]

Desimone, R. \& Duncan, J. (1995) Neural mechanisms of selective attention. Annual Review of Neuroscience 18:193-222. [aWAP, AR, PRR]

Dickerson, F. B. (2000) Cognitive behavioral psychotherapy for schizophrenia A review of recent empirical studies. Schizophrenia Research 16:71-90. [rSMS]

Dierks, T., Linden, D. E. J., Jandl, M., Formisano, E., Goebel, R., Lanfermann, H. \& Singer, W. (1999) Activation of Heschl's gyrus during auditory hallucinations. Neuron 22:615-21. [PRR]

Doheny, H. C., Faulkner, H. J., Gruzelier, J. H., Baldeweg, T. \& Whittington, M. A. (2000) Pathway-specific habituation of induced gamma oscillations in the hippocampal slice. NeuroReport 11:2629-33. [aWAP]

Dolan, R. J. Fletcher, P. C. McKenna, P., Friston, K. J. \& Frith, C. D. (1999) Abnormal neural integration related to cognition in schizophrenia. Acta Psychiatrica Scandinavica 99:58-67. [HPB, aWAP]

Donchin, E. \& Coles, M. G. H. (1988) Is the P300 component a manifestation of context updating. Behavioral and Brain Sciences 11:355-72. [DT]

Done, D. J. \& Frith, C. D. (1984) The effect of context during word perception in schizophrenic patients. Brain and Language 23:318-36. [DT]

Doniger, G. M., Silipo, G., Rabinowicz, E. F., Snodgrass, J. G. \& Javitt, D. C. (2001) Impaired sensory processing as a basis for object-recognition deficits in schizophrenia. American Journal of Psychiatry 158:1818-26. [DCJ]

Doody, G. A., Goetz, M., Johnson, E. C., Frith, C. D. \& Cunningham-Owens, D. G. (1998) Theory of mind and psychoses. Psychological Medicine 28:397-405. [DL]

Douglas, R. J. \& Martin, K. A. C. (1990) Neocortex. In: The synaptic organization of the brain, ed. G. M. Shepherd. Oxford University Press. [aWAP]

Dove, A., Pollmann, S., Schubert, T., Wiggins, C. J. \& von Cramon, Y. (2000) Prefrontal cortex activation in task switching: An event-related fMRI study. Cognitive Brain Research 9:103-109. [RDO]

Drury, V. M., Robinson, E. J. \& Birchwood, M. (1998) "Theory of mind" skills during acute episode of psychosis and following recovery. Psychological Medicine 28:1,101-12. [DL]

Duffy, L. \& O'Carroll, R. (1994) Memory impairment in schizophrenia - a comparison with that observed in the alcoholic Korsakoff syndrome. Psychological Medicine 24:155-65. [aWAP]

Duncan, E. J., Madonick, S. H., Parwani, A., Angrist, B., Rajan, R., Chakravorty, S., Efferen, T. R., Szilagyi, S., Stephanides, M., Chappell, P. B., Gonzenback, S., Ko, G. N. \& Rotrosen, J. P. (2001) Clinical and sensorimotor gating effects of ketamine in normals. Neuropsychopharmacology 25:72-83. [MEP, rSMS]

Duncan, G. E., Sheitman, B. B. \& Lieberman, J. A. (1999) An integrated view of pathophysiological models of schizophrenia. Brain Research Reviews 29:250_ 64. [aWAP]

Eastwood, S. L. \& Harrison, P. J. (1995) Decreased synaptophysin in the medial temporal lobe in schizophrenia demonstrated using immunoautoradiography. Neuroscience 69:339-43. [REH]

Edelman, G. M. (1989) The remembered present. Basic Books. [aWAP]

Ellison, G. (1995) The N-methyl-D-aspartate antagonists phencyclidine, ketamine and dizocilpine as both behavioral and anatomical models of the dementias. Brain Research Review 20:250-67. [aWAP]

Elvevag, B., Duncan, J. \& McKenna, P. (2000) The use of cognitive context in schizophrenia: An investigation. Psychological Medicine 30:885-97. [DRH]

Engel, A. K. \& Singer, W. (2001) Temporal binding and the neural correlates of sensory awareness. Trends in Cognitive Sciences 5:16-25. [aWAP]

Epstein, R. (2000) The neural-cognitive basis of the Jamesian stream of thought. Consciousness and Cognition 9:550-75. [DRH]
Fathollahi, Y. \& Salami, M. (2001) The role of N-methyl-D-aspartate receptors in synaptic plasticity of rat visual cortex in vitro: Effect of sensory experience. Neuroscience Letters 306:149-52. [GSS]

Faulkner, H. J., Traub, R. D. \& Whittington, M. A. (1998) Disruption of synchronous gamma oscillations in the rat hippocampal slice: A common mechanism of anaesthetic drug action. British Journal of Pharmacology 125:483-92. [aWAP]

(1999) Anaesthetic/amnesic agents disrupt beta frequency oscillations associated with potentiation of excitatory synaptic potentials in the rat hippocampal slice. British Journal of Pharmacology 128:1813-25. [aWAP]

Feierman, J. (1982) Nocturnalism: An ethological theory of schizophrenia. Medical Hypotheses 9:455-79. [GSS]

Feinberg, I. (1982/1983) Schizophrenia: Caused by a fault in programmed synaptic elimination? Journal of Psychiatric Research 17:319-34. [REH]

Feldman, D., Sherif, J. E., Press, W. A. \& Bear, M. F. (1990) N-methyl-Daspartate-evoked calcium uptake by kitten visual cortex maintained in vitro. Experimental Brain Research 80:252-59. [GSS]

Feldman, R. S., Meyer, J. S. \& Quenzer, L. F. (1997) Principles of neuropsychopharmacology. Sinauer. [aWAP]

Felleman, D. J. \& Van Essen, D. C. (1991) Distributed hierarchical processing in the primate cerebral cortex. Cerebral Cortex 1:1-47. [aWAP, PPR]

Field, D. J., Hayes, A. \& Hess, R. F. (1993) Contour integration by the human visual system: Evidence for a local “association field." Vision Research 33:173-93. [MEP]

Fisher, R. (1976a) On creative, psychotic, and ecstatic states. In: Readings in abnormal psychology: Contemporary perspectives, ed. L. R. Allman \& D. T. Jaffe. Harper and Row. (Original article published 1969). [aWAP] (1976b) On creative, psychotic, and ecstatic states: Update. In: Readings in abnormal psychology: Contemporary perspectives, ed. L. R. Allman \& D. T. Jaffe. Harper and Row. [aWAP]

Fodor, J. A. \& Pylyshyn, Z. W. (1988) Connectionism and cognitive architecture: A critical analysis. Cognition 28:3-72. [aWAP, rSMS]

Fonta, C., Chappert, C. \& Imbert, M. (2000) Effect of monocular deprivation on NMDAR1 immunostaining in ocular dominance columns of the marmoset Callithrix jacchus. Visual Neuroscience 17:345-52. [GSS]

Fox, K., Henley, J. \& Issac, J. (1999) Experience-dependent development of NMDA receptor transmission. Nature Neuroscience 2:297-99. [GSS]

Fox, K., Sato, H. \& Daw, N. W. (1990) The effect of varying stimulus intensity on NMDA-receptor activity in cat visual cortex. Journal of Neurophysiology 64:1413-28. [aWAP, PPR]

Franks, R. D., Adler, L. E., Waldo, M. C., Alpert, J. \& Freedman, R. (1983) Neurophysiological studies of sensory gating in mania: Comparison with schizophrenia. Biological Psychiatry 18:989-1005. [aWAP, rSMS]

Fries, P., Reynolds, J. H., Rorie, A. E. \& Desimone, R. (2001) Modulation of oscillatory neuronal synchronization by selective visual attention. Science 291:1560-63. [aWAP]

Friston, K. J. (1998) The disconnection hypothesis. Schizophrenia Research 30(2):115-25. [YC, DRH]

(1999) Schizophrenia and the disconnection hypothesis. Acta Psychiatrica Scandinavica 99:68-79. [HPB, aWAP]

Friston, K. J., Ungerleider, L. G., Jezzard, P. \& Turner R. (1995) Characterizing modulatory interactions between areas V1 and V2 in human cortex: A new treatment of functional MRI data. Human Brain Mapping 2:211-24. [aWAP]

Frith, C. D. (1992) The cognitive neuropsychology of schizophrenia. Erlbaum/ Taylor and Francis. [DL, aWAP]

Frith, C. D. \& Corcoran, R. (1996) Exploring theory of mind in people with schizophrenia. Psychological Medicine 26:521-30. [DL]

Frith, C. D., Stevens, M., Johnstone, E. C., Owens, D. G. C. \& Crow, T. (1983) Integration of schematic faces and other complex objects in schizophrenia. Journal of Nervous and Mental Disease 171:34-39. [aWAP]

Frith, U. \& Happé, F. (1994) Autism: Beyond "theory of mind." Cognition 50: 115-32. [aWAP]

Funahashi, M. \& Stewart, M. (1998) Properties of gamma-frequency oscillations initiated by propagating population bursts in retrohippocampal regions of rat brain slices. Journal of Physiology 510:191-208. [aWAP]

Fuster, J. M. (1997) The prefrontal cortex. Lippincott-Raven. [aWAP]

Gaddum, J. \& Hammeed, K. A. (1954) Drugs which antogonize 5hydroxytriptamine. British Journal of Pharmacology 9:240-48. [rSMS]

Gao, X-M., Sakai, K., Roberts, R. C., Conley, R. R., Dean, B. \& Tamminga, C. A. (2000) Ionotropic glutamate receptors and expression of N-methyl-daspartate receptor subunits in subregions of human hippocampus: Effects of schizophrenia. American Journal of Psychiatry 157:1141-49. [aWAP, rSMS]

Garey, L. J., Ong, W. Y., Patel, T. S., Kanani, M., Davis, A., Mortimer, A. M., Barnes, T. R. \& Hirsch, S. R. (1998) Reduced dendritic spine density on cerebral cortical pyramidal neurons in schizophrenia. Journal of Neurology, Neurosurgery and Psychiatry 65:446-53. [REH] 
Geary, D. C. \& Huffman, K. J. (2002) Brain and cognitive evolution: Forms of modularity and functions of mind. Psychological Bulletin 128:667-98. [GSS]

Geyer, M. A., Krebs-Thomson, K., Braff, D. L. \& Swerdlow, N. R. (2001) Pharmacological studies of prepulse inhibition models of sensorimotor gating deficits in schizophrenia: A decade in review. Psychopharmacology (Berlin) 156:117-54. [rSMS]

Ghonheim, M. M., Hinrichs, J. V., Mewaldt, S. P. \& Petersen, R. C. (1985). Ketamine: behavioral effects of subanesthetic doses. Journal of Clinical Psychopharmacology 5:70-77. [aWAP]

Giere, R. N. (2001) The nature and function of models. Behavioral and Brain Sciences 24:1060. [rSMS]

Gilbert, C. D. (1992) Horizontal integration and cortical dynamics. Neuron 9:1-13. [aWAP]

Gilbert, C. Ito, M., Kapadia, M. \& Westheimer, G. (2000) Interactions between attention, context and learning in primary visual cortex. Vision Research 40:1217-26. [aWAP, rSMS]

Glantz, L. A. \& Lewis, D. A. (1997) Reduction of synaptophysin immunoreactivity in the prefrontal cortex of subjects with schizophrenia. Regional and diagnostic specificity. Archives of General Psychiatry 54:660-69. [REH]

(2000) Decreased dendritic spine density on prefrontal cortical pyramidal neurons in schizophrenia. Archives of General Psychiatry 57:65-73. [REH]

Goff, D. C. \& Coyle, J. T. (2001) The emerging role of glutamate in the pathophysiology and treatment of schizophrenia. American Journal of Psychiatry 158:1367-77. [aWAP, rSMS]

Goff, D. C., Tsai, G., Levitt, J., Amico, E., Manoach, D., Schoenfeld, D. A., Hayden, D. L., McCaley, R. \& Coyle, J. T. (1999) A placebo-controlled trial of Dcycloserine added to conventional neuroleptics in patients with schizophrenia. Archives of General Psychiatry 56:21-27. [aWAP]

Gold, J. M., Carpenter, C. J., Randolph, C., Goldberg, T. \& Weinberger, D. R. (1997) Auditory working memory and Wisconsin Card Sort Test in schizophrenia. Archives of General Psychiatry 54:159-65. [aWAP]

Goldberg, T. E., Aloia, M. S., Gourovitch, M. L., Missar, D., Pickar, D., \& Weinberger, D. R. (1998) Cognitive substrates of thought disorder, I: The semantic system. American Journal of Psychiatry 155:1671-76. [aWAP]

Goldman-Rakic, P. S., Sclaidhe, S. P. O. \& Chafee, M. V. (2000) Domain specificity in cognitive systems. In: The new cognitive neurosciences, ed. M. S Gazzaniga. MIT Press. [aWAP, rSMS]

Goodarzi, M. A., Wykes, T. \& Hemsley, D. R. (2000) Cerebral lateralization of global-local processing in people with schizotypy. Schizophrenia Research 45:115-21. [aWAP]

Gooding, D. C. (1999) Antisaccade task performance in questionnaire-identified schizotypes. Schizophrenia Research 35:157-66. [DCG]

Gooding, D. C., Kwapil, T. R. \& Tallent, K. A. (1999) Wisconsin Card Sorting Test deficits in schizotypic individuals. Schizophrenia Research 40:201-209. [DCG]

Gooding, D. C., Miller, M. D. \& Kwapil, T. R. (2000) Smooth pursuit and visual fixation in psychosis-prone individuals. Psychiatry Research 93:41-54. [DCG]

Gooding, D. C., Tallent, K. A. \& Hegyi, J. V. (2001) Cognitive slippage in schizotypic individuals. Journal of Nervous and Mental Disease 189:750-56. [DCG]

Gopnik, A. \& Wellman, H. M. (1992) Why the child's theory of mind really is a theory. Special Issue: Mental simulation: Philosophical and psychological essays. Mind and Language 7:145-71. [DL]

Gordon, E., Williams, L. M., Haig, A. R., Wright, J. \& Meares, R. A. (2001) Symptom profile and "gamma" processing in schizophrenia. Cognitive Neuropsychiatry 6:7-19. [arWAP, LMW]

Gottesman, I. I. (1991) Schizophrenia genesis. W. H. Freeman. [DCG]

Grace, A. A. (1991) Phasic vs. tonic dopamine release and the modulation of dopamine system responsivity: A hypothesis for the etiology of schizophrenia. Neuroscience 41:1-24. [JPG]

Grafman, J., Partiot, A. \& Hollnagel, C. (1995) Fables of the prefrontal cortex. Behavioral and Brain Sciences 18:349-58. [aWAP]

Gray, C. M. (1999) The temporal correlation hypothesis of visual feature integration: Still alive and well. Neuron 24:31-47. [aWAP]

Gray, J. A., Feldon, J., Rawlins, J. N. P., Hemsley, D. R. \& Smith, A. D. (1991) The neuropsychology of schizophrenia. Behavioral and Brain Sciences 14:1-84. [DRH, SP, aWAP]

Gray, J. R., Braver, T. S. \& Raichle, M. E. (2002) Integration of emotion and cognition in the lateral prefrontal cortex. Proceedings of the National Academy of Sciences, USA 99(6):4115-20. [SP]

Graybiel, A. M. (1997) The basal ganglia and cognitive pattern generators Schizophrenia Bulletin 23:459-69. [aWAP]

Green, M. F., Nuechterlein, K. H., Breitmeyer, B. \& Mintz, J. (1999) Backward masking in unmedicated schizophrenic patients in psychotic remission: Possible reflection of aberrant cortical oscillation. American Journal of Psychiatry 156:1367-73. [RC, aWAP]

Green, M. F., Nuechterlein, K. H. \& Mintz, J. (1994) Backward masking in schizophrenia and mania: II. Specifying the visual channels. Archives of General Psychiatry 51:945-51. [RC]

Green, M. J., Williams, L. M. \& Hemsley, D. R. (2000) Cognitive theories of delusion formation: The contribution of visual scan path research. Cognitive Neuropsychiatry 5:63-74. [DRH]

Greengard, P., Nairn, A. C., Girault, J. A., Ouimet, C. C., Snyder, G. L., Fisone, G. Fienberg, A. \& Nishi, A. (1998) The DARPP-32/protein phosphatase-1 cascade: A model for signal integration. Brain Research Reviews 26:274-84 [aWAP]

Grossberg, S. (1976) Adaptive pattern classification and universal recoding, II: Feedback, expectation, olfaction, and illusions. Biological Cybernetics 23: 187-202. [SG]

(1978) Behavioral contrast in short-term memory: Serial binary memory models or parallel continuous memory models? Journal of Mathematical Psychology 3:199-219. [SG]

(1984) Some normal and abnormal behavioral syndromes due to transmitter gating of opponent processes. Biological Psychiatry 19:1075-118 [SG, LMW]

(1999) How does the cerebral cortex work? Learning, attention, and grouping by the laminar circuits of visual cortex. Spatial Vision 12:163-85. [SG, PRR]

(2000a) How hallucinations may arise from brain mechanisms of learning, attention, and volition. Journal of the International Neuropsychological Society 6:583-92. [SG]

(2000b) The complementary brain: Unifying brain dynamics and modularity. Trends in Cognitive Sciences 4:233-46. [SG]

(2000c) The imbalanced brain: From normal behavior to schizophrenia Biological Psychiatry 48:81-98. [SG]

Grossberg, S. \& Grunewald, A. (1997) Cortical synchronization and perceptual framing. Journal of Cognitive Neuroscience 9:117-32. [SG]

Grossberg, S., Mingolla, E. \& Viswanathan, L. (2001) Neural dynamics of motion integration and segmentation within and across apertures. Vision Research 41:2521-53. [SG]

Grossberg, S. \& Raizada, R. (2000) Contrast-sensitive perceptual grouping and object-based attention in the laminar circuits of primary visual cortex. Vision Research 40:1413-32. [SG]

Grossberg, S. \& Somers, D. (1991) Synchronized oscillations during cooperative feature linking in a cortical model of visual perception. Neural Networks 4:453-66. [SG]

Grunwald, T., Beck, H., Lehnertz, K., Blumcke, I., Pezer, N., Kurthen, M., Fernandez, G., Van Roost, D., Heinze, H. J., Kutas, M. \& Elger, C. E. (1999) Evidence relating human verbal memory to hippocampal N-methyl-Daspartate receptors. Proceedings of the National Academy of Sciences USA 96:12085-89. [DT]

Grunze, H. C. R., Rainnie, D. G., Hasselmoe, M. E., Barkai, E. Hearn, E. F., McCarley, R. W. \& Greene, R. W. (1996) NMDA-dependent modulation of CA1 local circuit inhibition. Journal of Neuroscience 16:2034-43. [aWAP]

Gruzelier, J. (2000) Self-regulation of electrocortical activity in schizophrenia and schizotypy: A review. Clinical Electroencephalography 31:23-29. [rSMS]

Gruzelier, J., Seymour, K., Wilson, L., Jolley, A. \& Hirsch, S. (1988) Impairments on neuropsychological tests of temporohippocampal and frontohippocampal functions and word fluency in remitting schizophrenia and affective disorders. Archives of General Psychiatry 45:623-29. [aWAP]

Guillem, F., Bicu, M., Hooper, R., Bloom, D., Wolf, M-A., Messier, J., Desautels, R. \& Debruille. J. B. (2001) Memory impairment in schizophrenia: A study using event-related potentials in implicit and explicit tasks. Psychiatry Research 104:157-173. [DT]

Guillem, F., Bicu, M., Hooper, R., Bloom, D., Wolf, M.-A., Messier, J. Desautels, R., Todorov, C., Lalonde, P., Debruille, J. B. (2003) The cognitive and anatomo-functional basis of reality distortion in schizophrenia: A view from memory event-related potentials. Psychiatry Research 111:137-58. [DT]

Gurd, J. M., Amunts, K., Weiss, P. H., Zafiris, O., Zilles, K., Marshall, J. C. \& Fink, G. R. (2002) Posterior parietal cortex is implicated in continuous switching between verbal fluency tasks: An fMRI study with clinical implications. Brain 125:1024-38. [RDO]

Gurwitsch, A. (1964) The field of consciousness. Duquesne University Press. [LAS, rSMS]

Habeck, C. G. \& Srinivasan, R. (2000) Neural solutions to the problem of functional integration. Behavioral and Brain Sciences 23:402-403. [aWAP]

Hachem, D. G., Vivian, P. J. \& Azouri, E. F. (1997) Retrospective study of 6,988 involuntary schizophrenic admissions and readmissions over a decade (19801990). Biological Psychiatry 42(1) (Suppl. 2):193S. [JPG]

Haenschel, C., Baldeweg, T., Croft, R. J., Whittington, M. \& Gruzelier, J. (2000) Gamma and beta frequency oscillations in response to novel auditory stimuli: A comparison of human electroencephalogram (EEG) data with in vitro models. Proceedings of the National Academy of Sciences USA 97:7645-50. [aWAP]

Halford, G., Wilson, W. H. \& Phillips, S. (1998) Relational complexity metric is effective when assessments are based on actual cognitive processes. 
Behavioral and Brain Sciences 21(6):848-64. http://www.bbsonline.org/ documents/a/00/00/05/50/bbs00000550-00/bbs.halford.html [DL]

Halgren, E. \& Smith, M. E. (1987) Cognitive evoked potentials as modulatory processes in human memory formation and retrieval. Human Neurobiology 6:129-39. [DT]

Hamilton, R. \& Pascual-Leone, A. (1998) Cortical plasticity associated with Braille learning. Trends in Cognitive Sciences 2:168-74. [GSS]

Happé, F. (1997) Central coherence and theory of mind in autism: Reading homographs in context. British Journal of Developmental Psychology 15:1-12 [aWAP]

Happé, F. (1999) Autism: Cognitive deficit or cognitive style? Trends in the Cognitive Sciences 3:216-22. [aWAP]

Harborne, G. C., Watson, F. L., Healy, D. T. \& Groves, L. (1996) The effects of subanesthetic doses of ketamine on memory, cognitive performance and subjective experience in healthy volunteers. Journal of Psychopharmacology 10:134-40. [aWAP]

Harrison, P. J. \& Eastwood, S. L. (2001) Neuropathological studies of synaptic connectivity in the hippocampal formation in schizophrenia. Hippocampus 11(5):508-19. [LMT]

Hasbani, M. J., Schlief, M. L., Fisher, D. A. \& Goldberg, M. P. (2001) Dendritic spines lost during glutamate receptor activation reemerge at original sites of synaptic contact. Journal of Neuroscience 21:2393-403. [REH]

Heinrichs, R. W. (1993) Schizophrenia and the brain. American Psychologist 48:221-33. [aWAP]

Hemby, S. E., Ginsberg, S. D., Brunk, B., Arnold, S. E., Trojanowski, J. Q. \& Eberwine, J. H. (2002) Gene expression profile for schizophrenia: Discrete neuron transcription patterns in the entorhinal cortex. Archives of General Psychiatry 59(7):631-40. [LMT]

Hemsley, D. R. (1975) A two stage model of attention in schizophrenia research. British Journal of Social and Clinical Psychology 14:81-88. [DRH]

(1994) Cognitive disturbance as the link between schizophrenic symptoms and their biological bases. Neurology, Psychiatry and Brain Research 2:163-70. [DRH]

(1998) The disruption of the "sense of self" in schizophrenia: Potential links with disturbances of information processing. British Journal of Medical Psychology 71:115-24. [DRH]

Hemsley, D. R., Rawlins, J. N. P., Feldon, J., Jones, S. H. \& Gray J. A. (1993) The neuropsychology of schizophrenia: Act 3. Behavioral and Brain Sciences 16:209-15. [DRH, RDO, aWAP]

Hemsley, D. R. \& Richardson, P. H. (1980) Shadowing by context in schizophrenia. Journal of Nervous and Mental Disease 168:141-45. [DRH]

Heresco-Levy, U., Javitt, D. C., Ermilov, M., Mordel, C., Silipo, G. \& Lichtenstein M. (1999) Efficacy of high-dose glycine in the treatment of enduring negative symptoms of schizophrenia. Archives of General Psychiatry 56:29-36. [aWAP, rSMS]

Hertzman, M., Reba, R. C. \& v.Kotlyarov, E. (1991). Single photon emission computed tomography in phencyclidine and related drug abuse. American Journal Psychiatry 147:256-57. [aWAP]

Hirsch, S. R., Das, I., Garey, L. J. \& Debelleroche, J. (1997) A pivotal role for glutamate in the pathogenisis of schizoprenia and its cognitive dysfunction. Pharmacology, Biochemistry and Behavior 56:797-802. [aWAP]

Hoffman, R. E., Buchsbaum, M. S., Jensen, R. V., Guich, S. M., Tsai, K. \& Nuechterlein, K. H. (1996) Dimensional complexity of EEG waveforms in neuroleptic-free schizophrenic patients and normal control subjects. Journal of Neuropsychiatry and Clinical Neurosciences 8:436-41. [aWAP]

Hoffman, R. E. \& Dobscha, S. (1989 Cortical pruning and the development of schizophrenia: A computer model. Schizophrenia Bulletin 15:477-90. [REH]

Hoffman, R. E. \& McGlashan, T. H. (1993) Parallel distributed processing and the emergence of schizophrenic symptoms. Schizophrenia Bulletin 19:119-40. [aWAP, rSMS]

(1997) Synaptic elimination, neurodevelopment and the mechanism of hallucinated "voices" in schizophrenia. American Journal of Psychiatry 154:1683-89. [REH]

(2001) Neural networks models of schizophrenia. The Neuroscientist 7:441-54. [REH]

Holcomb, H. H., Lahti, A. C., Medoff, D. R., Weiler, M. \& Tamminga, C. A (2001) Sequential regional cerebral blood flow brain scans using PET with $\mathrm{H} 2(15) \mathrm{O}$ demonstrate ketamine actions in CNS dynamically. Neuropsychopharmacology 25:165-72. [MAT]

Holcomb, P. J. (1993) Semantic priming and stimulus degradation: Implications for the role of the N400 in language processing. Psychophysiology 30:47-61. [DT]

Holzman, P. S. (1994) Parsing cognition. The power of psychology paradigms. Archives of General Psychiatry 51(12):952-54. [YC]

Horrobin, D. (1979) Schizophrenia: Reconciliation of the dopamine, prostaglandin, and opioid concepts and the role of the pineal. Lancet 1 (Issue 8115):529-31. [GSS]
Horwitz, B., Tagamets M. A. \& McIntosh A. R. (1999) Neural modeling, functional brain imaging, and cognition. Trends in Cognitive Sciences 3:91-98. [MAT]

Ibrahim, H. M., Hogg, A. J., Healy, D. J., Haroutunian, V., Davis, K. L. \& MeadorWoodruff, J. H. (2000) Ionotropic glutamate receptor binding and subunit mRNA expression in the schizophrenic thalamus. Biological Psychiatry 47: 22-28. [rSMS]

Ito, M. \& Gilbert, C. D. (1999) Attention modulates contextual influences in the primary visual cortex of alert monkeys. Neuron 22:593-604. [aWAP]

Izawa, R. \& Yamamoto, S. (2002) Spatio-temporal disintegration of visual perception in schizophrenia as revealed by a novel cognitive task, the Searchlight Test. Schizophrenia Research 52:67-74. [rSMS]

James, W. (1890) The principles of psychology. Macmillan. [DRH]

Janowsky, J. S., Shimamura, A. P. \& Squire, L. R. (1989) Source memory impairment in patients with frontal lobe lesions. Neuropsychologia 27 1043-56. [aWAP]

Jarrold, C., Butler, D. W., Cottington, E. M. \& Jiminez, F. (2000) Linking theory of mind and central coherence bias in autism and in the general population. Developmental Psychology 36:126-38. [rSMS]

Javitt, D. C., Shelley, A.-M. \& Riter, W. (2000) Associated deficits in mismatch negativity generation and tone matching in schizophrenia. Clinical Neurophysiology 111:1733-37. [RDO]

Javitt, D. C., Silipo, G., Cienfuegos, A., Shelley, A. M., Bark, N., Park, M. Lindenmayer, J. P., Suckow, R. \& Zukin, S. R. (2001) Adjunctive high-dose glycine in the treatment of schizophrenia. International Journal of Neuropsychopharmacology 4:385-92. [DCJ]

Javitt, D. C., Steinscheider, M., Schroeder, C. E. \& Arezzo, J. C. (1996) Role of cortical N-methyl-D-aspartate receptors in auditory sensory memory and mismatch negativity generation: Implications for schizophrenia. Proceedings of the National Academy of Science USA 93:11962-67. [aWAP, LMT, MEP]

Javitt, D. C. \& Zukin, S. R. (1989) Biexponential kinetics of $\quad[3 \mathrm{H}] \mathrm{MK}-801$ binding: Evidence for access to closed and open N-methyl-D-aspartate receptor channels. Molecular Pharmacology 35:387-93. [rSMS]

(1991) Recent advances in the phencyclidine model of schizophrenia. American Journal of Psychiatry 148:1301-308. [aWAP, rSMS]

Javoy-Agid, F., Scatton, B, Ruberg, M., L'Heureux, R., Cervera, P., Raisman, R., Maloteaux, J. M., Beck, H. \& Agid, Y. (1989) Distribution of monoaminergic, cholinergic, and GABAergic markers in the human cerebral cortex. Neuroscience 29:251-59. [LMW]

Jemel, B., Achenbach, C., Müller, B. W., Röpcke, B. \& Oades R. D. (2002) Mismatch negativity results from bilateral asymmetric dipole sources in the frontal and temporal lobes. Brain Topography 15:13-27. [RDO]

Jensen, O., Idiart, M. A. P. \& Lisman, J. E. (1996) Physiologically realistic formation of autoassociative memory in networks with theta/gamma oscillations: Role of fast NMDA channels. Learning and Memory 3:243-56. [aWAP]

Jensterle, J., Mlakar, J., Vodusek, D. B. \& Frith, C. D. (2000) Disorganization in schizophrenia need not result from a failure to inhibit dominant response tendencies. Cognitive neuropsychiatry 5:105-21. [DRH]

Jentsch, J. D., Redmond, Jr. D. E., Elsworth, J. D., Taylor, J. R., Youngren, K. D. \& Roth, R. H. (1997a) Enduring cognitive deficits and cortical dopamine dysfunction in monkeys after long-term administration of phencyclidine. Science 277:953-55. [aWAP]

Jentsch, J. D. \& Roth, R. H. (1999) The neuropsychopharmacology of phencyclidine: From NMDA receptor hypofunction to the dopamine hypothesis of schizophrenia. Neuropsychopharmacology 20:201-25. [aWAP, rSMS]

Jentsch, J. D., Tran, A., Le, D., Youngren, K. D. \& Roth, R. H. (1997b) Subchronic phencyclidine administration reduces mesoprefrontal dopamine utilization and impairs prefrontal cortical dependent cognition in the rat. Neuropsychopharmacology 17:92-99. [aWAP]

Jockers-Scherubl, M. C., Godeman, F. \& Pitzcker, A. (2001) Negative symptoms of schizophrenia are improved by paroxetine added to neuroleptics: A pilot study. Journal of Clinical Psychiatry 62:573. [RDO]

John, C. H. \& Hemsley, D. R. (1992) Gestalt perception in schizophrenia. European Archives of Psychiatry Clinical Neuroscience 241(4):215-21. [YC]

John, E. R. (2001) A field theory of consciousness. Consciousness and Cognition 10:184-213. [RC]

Johnson, S. C. \& Carey, S. (1998) Knowledge enrichment and conceptual change in folkbiology: Evidence from Williams syndrome. Cognitive Psychology $37(2): 156-200 . \quad[\mathrm{DL}]$

Jones, S. H., Hemsley, D. R. \& Gray, J. A. (1991) Contextual effects on choice reaction time and accuracy in acute and chronic schizophrenics. British Journal of Psychiatry 159:415-21. [aWAP]

Judd, L. L., McAdams, L., Budnick, B. \& Braff, D. L. (1992) Sensory gating deficits in schizophrenia: New results. American Journal of Psychiatry 149:488-93. [aWAP]

Kähkönen, S., Ahveninen, J., Pekkonen, E., Kaakkola, S., Huttunen, J., Ilmoniemi, R. J. \& Jääskiläinen, I. P. (2002) Dopamine modulates involuntary attention 
shifting and reorienting: An electromagnetic study. Clinical Neurophysiology 113:1894-1902. [RDO]

Kandel, E. R. (1998) A new intellectual framework for psychiatry. American Journal of Psychiatry 155:457-69. [aWAP]

Kapadia, M. K., Ito, M., Gilbert, C. D. \& Westheimer, G. (1995) Improvement in visual sensitivity by changes in local context: Parallel studies in human observers and in $\mathrm{V} 1$ of alert monkeys. Neuron 15:843-56. [aWAP, PPR]

Kaplan, R. D. (1974) The crossover phenomenon: Three studies of the effect of training and information on process schizophrenic reaction time. Unpublished doctoral dissertation, University of Waterloo, Ontario, Canada. [aWAP]

Kapur, S. \& Seeman, P. (2002) NMDA receptor antagonists ketamine and PCP have direct effects on the dopamine D2 and serotonin 5-HT2 receptors: Implications for models of schizophrenia. Molecular Psychiatry 7:837-44. [RDO, rSMS]

Karmiloff-Smith, A. (1998) Development itself is the key to understanding developmental disorders. Trends in the Cognitive Sciences 2:389-98. [aWAP]

Karp, H. N., Kaufman, N. D. \& Anand, S. K. (1980) Phencyclidine poisoning in young children. Journal of Pediatrics 97:1006-1009. [aWAP]

Karson, C. N., Mrak, R. E., Schluterman, K. O., Sturner, W. Q., Sheng, J. G. \& Griffin, W. S. (1999) Alterations in synaptic proteins and their encoding mRNAs in prefrontal cortex in schizophrenia: A possible neurochemical basis for "hypofrontality." Molecular Psychiatry 4:39-45. [REH]

Kay, J., Floreano, D. \& Phillips, W. A. (1998) Contextually guided unsupervised learning using local multivariate binary processors. Neural Networks 11:11740. [aWAP, rSMS]

Kay, S. R., Opler, L. A. \& Fiszbein, A. (1987) The positive and negative syndrome scale (PANNS) for schizophrenia. Schizophrenia Bulletin 13:261-76. [aWAP]

Kegeles, L. S., Abi-Dargham, A., Zea-Ponce, Y., Rodenhiser-Hill, J., Mann, J. J., Van Heertum, R. L., Cooper, T. B., Carlsson, A. \& Laruelle, M. (2000) Modulation of amphetamine-induced striatal dopamine release by ketamine in humans: Implications for schizophrenia. Biological Psychiatry 48:627-40. [DCJ]

Keil, A., Müller, M. M., Ray, W. J., Gruber, T. \& Elbert, T. (1999) Human gamma band activity and perception of a gestalt. Journal of Neuroscience 19:7152-61. [aWAP, rSMS]

Kelso, J. A. S. (1995) Dynamic patterns: The self-organization of brain and behavior. MIT Press. [aWAP]

Kendler, K. S., McGuire, M., Gruenberg, A. M., O’Hare, A., Spellman, M. \& Walsch D. (1993) The Roscommon family study. I. Methods, diagnosis of probands, and risk of schizophrenia in relatives. Archives of General Psychiatry 50:527-40. [REH]

Kim, D., Zemon, V., Saperstein, A., Butler, P. D. \& Javitt, D. C. (2002) Lateral cortical interactions in schizophrenia: A visual evoked potential study. Program No. 705.13. 2002 Abstract Viewer/Itinerary Planner. Society for Neuroscience, 2002. CD-ROM. [MEP]

Kisley, M. A., Olincy, A., Robbins, E., Polk, S. D., Adler, L. E., Waldo, M. C. \& Freedman, R. (2003) Sensory gating impairment associated with schizophrenia persists into REM sleep. Psychophysiology, 40:29-38. [MAK]

Kissler, J., Muller, M. M., Fehr, T., Rockstruh, B. \& Elbert, T. (2000) MEG gamma band activity in schizophrenia patients and healthy subjects in a mental arithmetic task and at rest. Clinical Neurophysiology 111:2079-87. [aWAP]

Klosterkötter, J., Schultze-Lutter, F., Gross, G., Huber, G. \& Steinmeyer, E. M. (1997) Early self-experienced neuropsychological deficits and subsequent schizophrenic diseases: An 8-year average follow-up prospective study. Acto Psychiatrica Scandinavica 95:396-404. [LAS]

Knierim, J. J. \& Van Essen, D. C. (1992) Neuronal responses to static texture patterns in area V1 of the alert macaque monkey. Journal of Neurophysiology 67:961-80. [PRR]

Knight, R. A. (1984) Converging models of cognitive deficit in schizophrenia. In: Theories of schizophrenia and psychosis. Nebraska Symposium on Motivation 1983, ed. W. D. Spaulding \& J. K. Cole. University of Nebraska Press. [aWAP, rSMS]

(1992) Specifying cognitive deficiencies in poor premorbid schizophrenics. In Progress in experimental personality and psychopathology research, vol. 15, ed. E. F. Walker, R. H. Dworkin \& B. A. Cornblatt. [aWAP, rSMS]

Knight, R. A., Elliot, D. S. \& Freedman, E. G. (1985) Short-term visual memory in schizophrenics. Journal of Abnormal Psychology 94:427-42. [aWAP]

Knight, R. A. \& Roff, J. D. (1985) Affectivity in schizophrenia: The predictive and discriminant validity of affectivity deficits. Chapter 15:280-317. In Controversies in schizophrenia: Changes and constancies, ed. M. Alpert. Erlbaum. [MA]

Knight, R. A. \& Silverstein, S. M. (1998) The role of cognitive psychology in guiding research on cognitive deficits in schizophrenia. In: Origins and development of schizophrenia: Advances in experimental psychopathology, ed. M. Lenzenweger \& R. H. Dworkin. APA Press. [aWAP, rSMS]

(2001) A process-oriented approach for averting confounds resulting from general performance deficiencies in schizophrenia. Journal of Abnormal Psychology 110:15-30. [rSMS]

Knight, R., Manoach, D. S, Elliott, D. S. \& Hershenson, M. (2001) Perceptual organization in schizophrenia: The processing of symmetrical configurations. Journal of Abnormal Psychology 109:575-87. [LAS]

Köhler, W. (1929) Gestalt psychology. Liveright. [rSMS]

(1947) Gestalt psychology: An introduction to new concepts in modern psychology. Liveright. [rSMS]

Konstantareas, M. M. \& Hewitt, T. (2001) Autistic disorder and schizophrenia: Diagnostic overlaps. Journal of Autism and Developmental Disorders 31:1928. [aWAP]

Kopell, N. (2000) We got rhythm: Dynamical systems of the nervous system. Notices of the AMS 47:6-16. [aWAP, rSMS, MAT]

Körding, K. P. \& König, P. (2000) Learning with two sites of synaptic integration. Network: Computation in Neural Systems 11:25-40. [aWAP]

Kovács, I. (1996) Gestalten of today: Early processing of visual contours and surfaces. Behavioral Brain Research 82:1-11. [aWAP]

Koyama, S., Nageishi, Y., Shimokochi, M., Hokama, H., Miyazato, Y., Miyatani, M. \& Ogura, C. (1991) The N400 component of event-related potentials in schizophrenic patients: A preliminary study. Electroencephalography and Clinical Neurophysiology 78:124-32. [DT]

Kreiter, A. K., \& Singer, W. (1996) Stimulus-dependent synchronization of neuronal responses in the visual cortex of the awake Macaque monkey. Journal of Neuroscience 16:2381-96. [aWAP]

Krystal, J. H., Bennett, A., Abi-Saab, W. M., Belger, A., Karper, L. P., D’Souza, D. C. Lipschitz, D., Abi-Dargham, A. \& Charney, D. S. (2000) Dissociation of ketamine effects on rule acquisition and rule implementation: Possible relevance to NMDA receptor contributions to executive cognitive functions. Biological Psychiatry 47:137-43. [aWAP]

Krystal, J. H., Karper, L. P., Seibyl, J. P., Freeman, G. K., Delaney, R., Bremner, J. D., Heninger, G. R., Bowers, M. B. \& Charney, D. S. (1994) Subanesthetic effects of the noncompetitive NMDA anatagonist, ketamine, in humans. Archives of General Psychiatry 51:199-214. [aWAP]

Kujala, T., Alho, K., Kekoni, J., Hamalainen, H., Rainikainen, K., Salonen, O., Standertskjold-Nordenstam, C. G. \& Naatanen, R. (1995) Auditory and somatosensory event-related brain potentials in early blind humans. Experimental Brain Research 104:519-26. [GSS]

Kuperberg, G. R., McGuire, P. K. \& David, A. S. (1998) Reduced sensitivity to linguistic context in schizophrenic thought disorder. Journal of Abnormal Psychology 107:423-34. [aWAP, rSMS]

Kutas, M. (1988) Review of event-related potentials studies of memory. In: Perspectives of memory research, ed. M. S. Gazzaniga. MIT Press. [DT]

Kutas M, McCarthy G, Donchin E. (1977) Augmenting mental chronometry: The P300 as a measure of stimulus evaluation time. Science 197(4305):792-95. [DT]

Kutsuwada, T., Kashiwabuchi, N., Mori, H., Sakimura, K., Kushiya, E., Araki, K. Meguro, H., Masaki, H., Kumanisis, T., Arakawa, M. \& Mishina, M. (1992) Molecular diversity of the NMDA receptor channel. Nature 358:36-41. [aWAP]

Kwapil, T. R. (1998) Social anhedonia as a predictor of the development of schizophrenia-spectrum disorders. Journal of Abnormal Psychology 107:55865. [DCG]

Kwon, J. S., O’Donnell, B. F., Wallenstein, G. V., Greene, R. W., Hirayasu, Y., Nestor, P. G., Hasselmo, M. E., Potts, G. F., Shenton, M. E. \& McCarley, R. W. (1999) Gamma-frequency range abnormalities to auditory stimulation in schizophrenia. Archives of General Psychiatry 56:1001-1005. [MAK, aWAP, rSMS]

Kwon, Y. H., Nelson, S. B., Toth, L. J. \& Sur, M. (1992) Effect of stimulus contrast and size on NMDA receptor activity in cat lateral geniculate nucleus. Journal of Neurophysiology 68:182-96. [DCJ]

Lahti, A. C., Holcomb, H. H., Medoff, D. R. \& Tamminga, C. A. (1995a) Ketamine activates psychosis and alters limbic blood-flow in schizophrenia. NeuroReport 6:869-72. [aWAP]

Lahti, A. C., Koffel, B., Laporte, D. \& Tamminga, C. A. (1995b) Subanesthetic doses of ketamine stimulate psychosis in schizophrenia. Neuropsychopharmacology 13:9-19. [aWAP]

Lamme, V. A. F. \& Roelfsema, P. R. (2000) The distinct modes of vision offered by feedforward and recurrent processing. Trends in Neuroscience 23:571-79. [PRR]

Lamme, V. A. F. \& Spekreijse, H. (2000) Contextual modulation in primary visual cortex and scene perception. In: The new cognitive neurosciences, ed. M. S. Gazzaniga. MIT Press. [aWAP]

Lamme, V. A. F., Zipser, K. \& Spekreijse, H. (1998) Figure-ground activity in primary visual cortex is suppressed by anesthesia. Proceedings of the National Academy of Sciences USA 95:3263-68. [PRR]

Lee, J., Chun, M. M., Jiang, Y. \& Park, S. (2003a) Spatial context effects on visuospatial working memory (VSWM) in schizophrenia. Schizophrenia Research 605:143. [SP] 
Lee, J. \& Park, S. (2002a) The role of stimuli-driven attention in spatial working memory in schizophrenia. Schizophrenia Research 53(3):131. [SP]

(2002b) The effect of target salience on the Continuous Performance Task (CPT-AX) performance in schizophrenia patients. Proceedings of the Seventeenth Annual Meeting of Society for Research in Psychopathology, San Francisco, CA p. 20. [SP]

Lee, K.-H., Williams, L. M., Breakspear, M. \& Gordon, E. (2003b) Synchronous Gamma activity: A review and contribution to an integrative neuroscience model of schizophrenia. Brain Research Reviews 41:57-78. [aWAP, LMW]

Lee, K.-H., Williams, L. M., Haig A. \& Gordon, E. (2003c) “Gamma (40Hz) phase synchronicity" and symptom dimensions in schizophrenia. Cognitive Neuropsychiatry 8:57-71. [LMW]

Lehar, S. (2002) The world in your head: A gestalt view of the mechanism of conscious experience. Erlbaum. [rSMS]

(in press) Gestalt isomorphism and the primacy of subjective conscious experience: A gestalt bubble model. Behavioral and Brain Sciences. [rSMS]

Leiser, D. (2001) Scattered naive theories: Why the human mind is isomorphic to the internet web. New Ideas in Psychology 19(3):175-202 http://www.bgu.ac.il/ dleiser/docs/naive.pdf [DL]

Lewis, D. A. \& Gonzalez-Burgos, G. (2000) Intrinsic excitatory connections in the prefrontal cortex and the pathophysiology of schizophrenia. Brain Research Bulletin 52:309-17. [REH]

Lezak, M. (1995) Neuropsychological assessment, 3rd edition. Oxford University Press. [aWAP]

Liddle, P. F. (1987) The symptoms of chronic schizophrenia. A re-examination of the positive-negative dichotomy. British Journal of Psychiatry 151:145-51. [aWAP]

Liddle, P. F., Friston, K. J., Frith, C. D., Hirsch, S. R., Jones, T. \& Frakowiak, R. S. J (1992) Patterns of cerebral blood flow in schizophrenia. British Journal of Psychiatry 160:179-86. [aWAP]

Liddle, P. F. \& Morris, D. L. (1991) Schizophrenic syndromes and frontal lobe performance. British Journal of Psychiatry 158:340-45. [aWAP]

Lindenmayer, J.-P., Bernstein-Hyman, R. \& Grochowski, S. (1994) A new five factor model of schizophrenia. Psychiatric Quarterly 65:299-322. [aWAP rSMS]

Liotti, M., Ryder, K. \& Woldorff, M. G. (1998) Auditory attention in the congenitally blind: Where, when and what gets reorganized? NeuroReport 9:1007-12. [GSS]

Lisman, J. E., Fellous, J. M. \& Wang, X. J. (1998) A role for NMDA-receptor channels in working memory. Nature Neuroscience 1:273-75. [aWAP, rSMS]

Lisman, J. E. \& Idiart, M. A. P. (1995) Storage of 7+/- 2 short-term memories in oscillatory subcycles. Science 267:1512-14. [aWAP]

Liu, D., Diorio, J., Day, J. C., Francis, D. D. \& Meaney, M. J. (2000) Maternal care, hippocampal synaptogenesis, and cognitive development in rats. Nature Neuroscience 3:799-806. [rSMS]

Llinas, R. (1993) Is dyslexia a dyschronia? Annals of the New York Academy of Sciences 682:48-56. [RC]

Lovell, P. G. (2002) Human contour integration: Evaluating the association field theory using psychophysical and computational methods. Submitted doctoral dissertation, University of Stirling, UK. Available via Internet at: http://www.stir.ac.uk/Departments/HumanSciences/Psychology/Postgrads/ pgll/downloads/PhDThesis/Thesis_MaxCompression.pdf [MEP]

Lubow, R. E. (1989) Latent inhibition and conditioned attention theory. Cambridge University Press. [DRH]

Lubow, R. E., Kaplan, O., Abramovich, P., Rudnick, A. \& Laor, N. (2000) Visual search in schizophrenia: Latent inhibition and novel pop-out effects. Schizophrenia Research 45:145-56. [PRR]

Luby, E. D., Gottlieb, J. S., Cohen, B. D. \& Rosenbaum, G. (1962) Model psychoses and schizophrenia. American Journal of Psychiatry 119:61-67. [aWAP]

Luck, S. J. \& Vogel, E. K. (1997) The capacity of visual working memory for features and conjunctions. Nature 390:279-81. [aWAP]

Lutz, A. (2002) Toward a neurophenomenology as an account of generative passages: A first empirical case study. Phenomenology and the Cognitive Sciences 1:133-67. [rSMS]

MacDonald, A. W., III, Pogue-Geile, M. F., Johnson, M. K. \& Carter, C. S. (2003) A specific deficit in context processing in the unaffected siblings of patients with schizophrenia. Archives of General Psychiatry 60:57-65. [AWM, rSMS]

Magaro, P. A. (1980) Cognition in schizophrenia and paranoia: The integration of cognitive processes. Erlbaum. [aWAP]

Maher, B. A., Manschreck, T. C. \& Rucklos, M. E. (1980) Contextual constraint and the recall of verbal material in schizophrenia: The effect of thought disorder. British Journal of Psychiatry 137:69-73. [aWAP]

Malhotra, A. K., Pinals, D. A., Adler, C. M., Elman, I., Clifton, A., Pickar, D. \& Breier, A. (1997) Ketamine-induced exacerbation of psychotic symptoms and cognitive impairment in neuroleptic-free schizophrenics.

Neuropsychopharmacology 17:141-50. [aWAP]
Malhotra, A. K., Pinals, D. A., Weingartner, H., Sirocco, K., Missar, C. D., Pickar, D. \& Breier, A. (1996) NMDA receptor function and human cognition - The effects of ketamine in healthy volunteers. Neuropsychopharmacology 14:301307. [aWAP]

Marenco, S. \& Weinberger, D. R. (2000) The neurodevelopmental hypothesis of schizophrenia: Following a trail of evidence from cradle to grave. Development and Psychopathology, 12:501-27. [RC]

Marr, D. (1982) Vision. W. W. Freeman. [rSMS]

Mathe, J. M., Nomikos, G. G., Schilstrom, B. \& Svensson, T. H. (1998) NonNMDA excitatory amino acid receptors in the ventral tegmental area mediate systemic dizocilpine (MK-801) induced hyperlocomotion and dopamine release in the nucleus accumbens. Journal of Neuroscience Research 51:58392. [rSMS]

Matussek, P. (1952/1987) Untersuchungen über die Wahnwahrnehmung [Studies in delusional perception]. Archiv für Psychiatry und Zeitschrift Neurologie 189:279-318. Translated and condensed English edition, 1987. In: Clinical roots of the schizophrenia concept. Translations of seminal European contributions on schizophrenia, ed. J. Cutting \& M. Sheppard. Cambridge University Press. [aWAP, LAS, rSMS]

Maunsell, J. H. R. (1995) The brain's visual world: Representations of visual targets in cerebral cortex. Science 270:764-68. [aWAP]

Mayes, A. R., Meudell, P. R. \& Pickering, A. D. (1985) Is organic amnesia caused by a selective deficit in remembering contextual information? Cortex 21:167202. [DRH]

McAdams, C. J. \& Maunsell, J. H. R. (1999) Effects of attention on orientationtuning functions of single neurons in macaque area V4. Journal of Neuroscience 19:431-41. [PRR]

McBain, C. J. \& Mayer, M. L. (1994) N-Methyl-D-Aspartic acid receptor structure and function. Physiological Review 74:723-63. [aWAP]

McCarley, R. W., Hsiao, J., Freedman, R., Pfefferbaum, A. \& Donchin, E. (1996) Neuroimaging and the cognitive neuroscience of schizophrenia. Schizophrenia Bulletin 22:703-26. [rSMS]

McCarley, R. W., Niznikiewicz, M. A., Salisbury, D. F., Nesotr, P. G., Salisbury, D. F., O’Donnell, B. F., Hirayasu, Y., Grunze, H., Greene, R. W. \& Shenton, M. E. (1999) Cognitive dysfunction in schizophrenia: Unifying basic research and clinical aspects. European Archives of Psychiatry and Clinical Neuroscience 249(Suppl. 4):69-82. [DCG]

McDonald, L. M., Moran, P. M., Vythelingum, M. H., Joseph, J. H., Stephenson, J. D \& Gray, J. A. (2002) Latent inhibition is attenuated by noise and restored by a 5-HT2A receptor antagonist. Behavioural Pharmacology 13:663-67. [RDO]

McGhie, A. \& Chapman, J. (1961) Disorders of attention and perception in early schizophrenia. British Journal of Medical Psychology 34:103-16. [rSMS]

McGlashan, T. H. \& Hoffman, R. E. (2000) Schizophrenia as a disorder of developmentally-reduced synaptic connectivity. Archives of General Psychiatry 57:637-48. [aWAP, REH]

Meador-Woodruff, J. H. \& Healy, D. J. (2000). Glutamate receptor expression in schizophrenic brain. Brain Research Reviews 31:288-94. [aWAP]

Mechri, A., Saoud, M., Khiari, G., d'Amato, T., Dalery, J. \& Gaha, L. (2001) Glutaminergic hypothesis of schizophrenia: studies with ketamine. Encephale 27:53-59. [aWAP]

Medalia, A., Aluma, M., Tryon, W. \& Merriam, A. E. (1998) Effectiveness of attention training in schizophrenia. Schizophrenia Bulletin 24:147-52. [rSMS]

Mednick, S. A. \& Silverton, L. (1988) High-risk studies of the etiology of schizophrenia. In: Handbook of schizophrenia: Nosology, epidemiology, and genetics of schizophrenia, vol. 3, ed. M. T. Tsuang \& J. C. Simpson. Elsevier. [aWAP]

Medoff, D. R., Holcomb, H. H., Lahti, A. C. \& Tamminga, C. A. (2001) Probing the human hippocampus using RCBF: Contrasts in schizophrenia. Hippocampus 11:543-50. [MAT]

Meehl, P. E. (1962) Schizotaxia, schizotypy, schizophrenia. American Psychologist 17:827-38. [DCG, rSMS]

(1989) Schizotaxia revisited. Archives of General Psychiatry 46:935-44. [DCG]

Meeter, M., Murre, J. M. J. \& Talamini, L. M. (2002) A computational approach to memory deficits in schizophrenia. Neurocomputing 44:929-36. [LMT]

Menninger, K., Mayman, M. \& Pruyser P. (1963) The vital balance. The Viking Press. [HPB]

Michie, P. T. (2001) What has MMN revealed about the auditory system in schizophrenia? International Journal of Psychophysiology 42:177-94. [RDO]

Miller, C. L., Bickford, P. C., Luntz-Leybman, V., Adler, L. E., Gerhardt, G. A. \& Freedman, R. (1992) Phencyclidine and auditory sensory gating in the hippocampus of the rat. Neuropharmacology 31:1041-48. [MAK]

Miller, E. K. \& Cohen, J. D. (2001) An integrative theory of prefrontal cortical function. Annual Review of Neuroscience 24:167-202. [aWAP]

Milner, R. (1999) Communicating and mobile systems: The $\pi$ calculus. Cambridge University Press. [HPB] 
Mintz, S. \& Alpert, M. (1972) Imagery vividness, reality testing and schizophrenic hallucinations. Journal of Abnormal Psychology 79(3):310-16. [MA]

Miyake, A., Friedman, N. P., Emerson, M. J., Witzki, A. H. \& Howerter, A. (2000) The unity and diversity of executive functions and their contributions to complex "frontal lobe" tasks: A latent variable analysis. Cognitive Psychology 41:49-100. [DCG]

Moghaddam, B., Adams, B., Verma, A. \& Daly, D. (1997) Activation of glutamatergic neurotransmission by ketamine - A novel step in the pathway from NMDA receptor blockade to dopaminergic and cognitive disruptions associated with the prefrontal cortex. Journal of Neuroscience 17:2921-27. [aWAP]

Mohn, A. R., Gainetdinov, R. R., Caron, M. G. \& Koller, B. H. (1999) Mice with reduced NMDA receptor expression display behaviors related to schizophrenia. Cell 98:427-36. [rSMS]

Møller, P. \& Husby, R. (2000) The initial prodrome in schizophrenia: Searching for naturalistic core dimensions of experience and behavior. Schizophrenia Bulletin 26:217-32. [LAS]

Monaghan, D. T., Bridges, R. J., \& Cotman, C. (1989) The excitatory amino acid receptors: Their classes, pharmacology, and distinct properties in the function of the central nervous system. Annual Review of Pharmacology and Toxicology 29:365-402. [aWAP]

Monchi, O., Taylor, J. G. \& Dagher, A. (2002) A neural model of working memory processes in normal subjects, Parkinson's disease and schizophrenia for fMRI design and predictions. Neural Networks 13:953-73. [LMT]

Monyer, H., Burnashev, N., Laurie, D. J., Sakmann, B. \& Seeburg, P. H. (1994) Developmental and regional expression in the rat brain and functional properties of four NMDA receptors. Neuron 12:529-40. [aWAP]

Moran, P. M., Al-Uzri, M. M., Watson, J. \& Reveley, M. A. (2003) Reduced Kamin blocking in non-paranoid schizophrenia: Associations with schizotypy. Journal of Psychiatric Research 37:155-63. [RDO]

Murthy, V. N. \& Fetz, E. E. (1996) Synchronization of neurons during local field potential oscillations in sensorimotor cortex of awake monkeys. Journal of Neurophysiology 76:3968-82. [aWAP]

Näätänen, R. (1990) The role of attention in auditory information processing as revealed by event-related potentials and other brain measures of cognitive function. Behavioral and Brain Sciences 13:201-88. [RDO]

Nadler, V., Kloog, Y. \& Sokolovsky M. (1990) Distinctive structural requirement for the binding of uncompetitive blockers (phencyclidine-like drugs) to the NMDA receptor. European Journal of Pharmacology 188:97-104. [rSMS]

Nakagawa, H., Sato, K., Shiraishi, Y., Kuriyama, H. \& Altschuler, R. A. (2000) NMDAR 1 isoforms in the rat superior olivary complex and changes after unilateral cochlear ablation. Brain Research. Molecular Brain Research 77:246-57. [GSS]

Neville, H., Kutas, M., Chesney, G. \& Schmidt, A. L. (1986) Event-related brain potentials during initial encoding and recognition memory of congruous and incongruous words. Journal of Memory and Language 25:75-92. [DT]

Newcomer, J. W., Farber, N. B., Jevtovic-Todorovic, V., Selke, G., Melson, A. K., Hershley, T., Craft, S. \& Olney, J. W. (1998) Ketamine-induced NMDA receptor hypofunction as a model of memory impairment and psychosis. Neuropsychopharmacology 20:106-18. [aWAP]

Newcomer, J. W. \& Krystal, J. H. (2001) NMDA receptor regulation of memory and behavior in humans. Hippocampus 11:529-42. [LMT]

Niebur, E. (2001) Research, robots, and reality: A statement on current trends in biorobotics. Behavioral and Brain Sciences 24:1072-73. [rSMS]

Nishijo, H., Yamamoto, Y., Ono, T., Uwano, T., Yamashita, J. \& Yamashita, T. (1997) Single neuron responses in the monkey anterior cingulate cortex during visual discrimination. Neuroscience Letters 227:79-82. [GSS]

Niznikiewicz, M. A., Voglmaier, M., Shenton, M. E., Seidman, L. J., Dickey, C. C. Rhoads, R., Teh, E. \& McCarley, R. W. (1999) Electrophysiological correlates of language processing in schizotypal personality disorder. American Journal of Psychiatry 56:1052-58. [DT]

Nuechterlein, K. H. (1977). Reaction time and attention in schizophrenia: A critical evaluation of the data and theories. Schizophrenia Bulletin 3:373-428. [aWAP]

Nuechterlein, K. H., Dawson, M. E., Gitlin, M., Ventura, J., Goldstein, M. J., Snyder, K. S., Yee, C. M. \& Mintz, J (1992). Developmental processes in schizophrenic disorders: Longitudinal studies of vulnerability and stress. Schizophrenia Bulletin 18:387-424. [aWAP]

Nunez, P. L. (2000) Towards a quantitative description of large-scale neocortical dynamic function and EEG. Behavioral and Brain Sciences 23:371-473. [aWAP]

Oades, R. D. (1985) The role of noradrenaline in tuning and dopamine in switching between signals in the CNS. Neuroscience and Biobehavioral Reviews 9:261-83. [RDO]

Oades, R. D., Müller, B., Schall U., Bender, S. \& Wolstein, J. (2000) Automatic vs. controlled attention in schizophrenia: Conditioned blocking and sensory gating. Behavioural Pharmacology 11:346. [RDO]

Oades, R. D., Rivet, J.-M., Taghzouti, K., Kharouby, M., Simon, H. \& Le Moal, M. (1987) Catecholamines and conditioned blocking: Effects of ventral tegmental, septal and frontal 6-hydroxydopamine lesions in rats. Brain Research 406:136-46. [RDO]

Oades, R. D., Ropcke, B. \& Eggers, C. (1994) Monoamine activity reflected in urine of young patients with obsessive compulsive disorder, psychosis with and without reality distortion and healthy subjects: An explorative analysis. Journal of Neural Transmission - General Section 96:143-59. [LMW]

Oades R. D., Zerbin, D. \& Eggers, C. (1993) Stimulus-Vergleichsprozesse bei psychotischen Jugendlichen mit paranoiden und nicht paranoiden Symptomen: Mismatch Negativity deutet auf differenzierte Beeinträchtigungen hin. In: Biologische Psychiatrie der Gegenwart, ed. P. Baumann. Springer-Verlag. [RDO]

Oades, R. D., Zimmermann, B. \& Eggers, C. (1996) Conditioned blocking in patients with paranoid, non-paranoid psychosis or obsessive compulsive disorder: Associations with symptoms, personality and monoamine metabolism. Journal of Psychiatric Research 30:369-90. [RDO]

Oleskevich, S. \& Walmsley, B. (2002) Synaptic transmission in the auditory brainstem of normal and congenitally deaf mice. The Journal of Physiology 540:447-55. [GSS]

Olney, J. W. \& Farber, N. B. (1995) Glutamate receptor dysfunction and schizophrenia. Archives of General Psychiatry 52:998-1007. [aWAP]

Olney, J. W., Newcomer, J. W. \& Farber, N. B. (1999) NMDA receptor hypofunction model of schizophrenia. Journal of Psychiatric Research 33:523-33. [aWAP]

Olufsen, M. S., Whittington, M. A., Camperi, M. \& Kopell, N. (2003) New roles for the gamma rhythm: Population tuning and preprocessing for the beta rhythm. Journal of Computational Neuroscience 14:33-54. [rSMS]

Oranje, B., Gispen-de Weid, C. C., Verbaten, M. N. \& Kahn, R. S. (2002) Modulating sensory gating in healthy volunteers: The effects of ketamine and haloperidol. Biological Psychiatry 52:887-95. [MAK, rSMS]

O’Reilly, R. C. \& Rudy, J. W. (2001) Conjunctive representations in learning and memory: Principles of cortical and hippocampal function. Psychological Review 108:311-45. [DRH]

Osborn, L. M., Silverstein, S. M., Matteson, S., West, L., Kamin, D. \& Schwarzkopf, S. B. (1994) Visual organization, memory, and thought disorder in schizoprenia. Biological Psychiatry 35:633. [aWAP]

Owen, A. M. (2000) The role of the lateral frontal cortex in mnemonic processing: The contribution of functional neuroimaging. Experimental Brain Research 133:33-43. [aWAP]

Pack, C. C., Berezovskii, V. K. \& Born, R. T. (2001) Dynamic properties of neurons in cortical area MT in alert and anaesthetized macaque monkeys. Nature 414:905-908. [PRR]

Pani, J. R., Mervis, C. B. \& Robinson B. F. (1999). Global spatial organization by individuals with Williams Syndrome. Psychological Science 10:453-58. [aWAP]

Park, S. \& Holzman, P. S. (1992) Schizophrenics show spatial working memory deficits. Archives of General Psychiatry 49:975-82. [aWAP]

Park, S., Jefferis, V., Knurek, E., Pitiyanuvath, N. \& Brown, N. (2000) Facilitation of prefrontal function in schizophrenia by increasing socioaffective input. Biological Psychiatry 47(8):S67-68. [SP]

Parnas, J., Jansson, L., Sass, L. A. \& Handest, P. (1998) Self-experience in the prodromal phases of schizophrenia: A pilot study of first admissions. Neurology, Psychiatry, and Brain Research 6:97-106. [LAS]

Parnas, J., Vianin, P., Saebye, D., Jansson, L., Volmer-Larsen, A. \& Bovet, P. (2001) Visual binding abilities in the initial and advanced stages of schizophrenia. Acta Psychiatrica Scandinavica 103:171-80. [rSMS]

Passingham, R. E., Stephan, K. E. \& Kotter R. (2002) The anatomical basis of functional localization in the cortex. Nature Reviews Neuroscience 3:606-16. [SLB]

Patterson, T. (1987) Studies toward the subcortical pathogenesis of schizophrenia. Schizophrenia Bulletin 13:555-76. [aWAP]

Patterson, T., Spohn, H. E., Bogia. D. P. \& Hayes, K. (1986) Thought disorder in schizophrenia: Cognitive and neuroscience approaches. Schizophrenia Bulletin 12:460-72. [aWAP]

Paul, G. L. \& Lentz, R. J. (1977) Psychosocial treatment of chronic mental patients Milieu versus social learning programs. Harvard University Press. [rSMS]

Pekkone, E., Hirvonen, J., Ahveninen, J., Kahkonen, S., Kaakola, S., Huttunen, J. \& Jaaskelainen, I. P. (2002) Memory-based comparison process not attenuated by haloperidol: A combined MEG and EEG study. NeuroReport 13:177-81. [MEP]

Perlstein, W. M., Carter, C. S., Barch, D. M. \& Baird, J. W. (1998) The Stroop task and attention deficits in schizophrenia. Neuropsychology 12:414-25. [aWAP]

Perlstein, W. M., Carter, C. S., Noll, D. C. \& Cohen, J. D. (2001) Relation of prefrontal cortex dysfunction to working memory and symptoms in schizophrenia. American Journal of Psychiatry 158:1105-13. [aWAP]

Perry, W., Geyer, M. A. \& Braff, D. L. (1999) Sensorimotor gating and thought disturbance measured in close temporal proximity in schizophrenic patients. Archives of General Psychiatry 56:277-81. [aWAP] 
Petrides, M. (1994) Frontal lobes and behavior. Current Opinion in Neurobiology 4:207-11. [aWAP]

Phillips, W. A. (1974) On the distinction between sensory storage and short-term visual memory. Perception and Psychophysics 16:283-90. [aWAP]

(2001) Contextual modulation and dynamic grouping in perception: Response. Trends in Cognitive Sciences 5:95-96. [rSMS]

Phillips, W. A. \& Christie, D. F. M. (1977) Interference with visualization. Quarterly Journal of Experimental Psychology 29:637-50. [aWAP]

Phillips, W. A. \& Craven, B. J. (2000) Interactions between coincident and orthogonal cues to texture boundaries. Perception and Psychophysics 62:1019-38. [rSMS]

Phillips, W. A., Kay, J. \& Smyth, D. (1995) The discovery of structure by multistream networks of local processors with contextual guidance. Network 6:22546. [AWM, aWAP, rSMS]

Phillips, W. A. \& Pflieger, M. E. (2000) Interactions that coordinate cortical activity. In: Integrated human brain science: Theory, method, application (music), ed. T. Nakada. Elsevier. [MEP, aWAP]

Phillips, W. A. \& Singer, W. (1997a) In search of common foundations for cortical computation. Behavioral and Brain Sciences 20:657-722. [DRH, MEP, aWAP, PPR, rSMS, MAT]

(1997b) Progress towards an understanding of cortical computation. Behavioral and Brain Sciences 20:703-22. [aWAP, rSMS]

Pickering, A. D. (1993) Schizophrenia: In context or in the garbage can? Behavioral and Brain Sciences 16:205-206. [aWAP]

Picton, T. W., Alain, C., Otten, L., Ritter, W. \& Achim, A. (2000) Mismatch negativity: Different water in the same river. Audiology and Neurootology 5:111-39. [MAK

Pigache, R. M. (1999) Vigilance in schizophrenia and its disruption by impaired preattentive selection: A dysintegration hypothesis. Cognitive Neuropsychiatry 4:119-44. [aWAP]

Pilling, S., Bebbington, P., Kuipers, E., Garety, P., Geddes, J., Orbach, G. \& Morgan, C. (2002) Psychological treatments in schizophrenia: I. Meta-analysis of family intervention and cognitive behaviour therapy. Psychological Medicine 32:763-82. [rSMS]

Place, E. J. S. \& Gilmore, G. C. (1980) Perceptual organization in schizophrenia Journal of Abnormal Psychology 89:409-18. [YC, AWM, aWAP, rSMS]

Polat, U., Mizobe, K., Pettet, M. W., Kasamatsu, T. \& Norcia, A. M. (1998) Collinear stimuli regulate visual responses depending on cell's contrast threshold. Nature 391:580-84. [aWAP]

Polat, U. \& Norcia, A. M. (1996) Neurophysiological evidence for contrast dependent long range facilitation and suppression in the human visual cortex. Vision Research 36:2099-109. [MEP]

Polat, U. \& Sagi D. (1993) Lateral interactions between spatial channels: Suppression and facilitation revealed by lateral masking experiments. Vision Research 33:993-99. [aWAP]

Polat, U., Sagi, D. \& Norcia, A. M. (1997) Abnormal long-range spatial interactions in amblyopia. Vision Research 37:737-44. [MEP, aWAP]

Posner, M. I. \& Raichle, M. E. (1994) Images of mind. Freeman. [aWAP]

Povinelli, D. J. \& Preuss, T. M. (1995) Theory of mind: Evolutionary history of a cognitive specialization. Trends in Neurosciences. Special Issue: Evolution and Development of the Cerebral Cortex 18(9):418-24. [DL]

Premack, D. \& Woodruff, G. (1978) Does the chimpanzee have a theory of mind? Behavioral and Brain Sciences 4:515-26. [DL]

Prentky, R. A. (1979) Creativity and psychopathology. A neurocognitive perspective. In: Progress in experimental personality research, vol. 9, ed. B. A. Maher. Academic Press. [SP]

Pulvermuller, F. (1999) Words in the brain's language. Behavioral and Brain Sciences 22:253-336. [RC]

Quinlan, E. M., Philpot, B. D., Huganir, R. L. \& Bear, M. F. (1999) Rapid, experience-dependent expression of synaptic NMDA receptors in visual cortex in vivo. Nature Neuroscience 2:352-57. [GSS]

Rabinowicz, E. F., Knight, R. A., Bruder, G., Owen, D. R. \& Gorman, J. (1995) Short-term visual memory deficits in schizophrenia: Medication effects and electrophysiological correlates. Poster session presented at the meeting of the Society for Research in Psychopathology, October, 1995, Iowa City, IA. [aWAP]

Rabinowicz, E. F., Opler, L. A., Owen, D. R. \& Knight, R. A. (1996) The dot enumeration perceptual organization task (DEPOT): Evidence for a shortterm visual memory deficit in schizophrenia. Journal of Abnormal Psychology 105:336-48. [aWAP]

Rabinowicz, E. F., Silipo, G., Goldman, R. \& Javitt, D. C. (2000) Auditory sensory dysfunction in schizophrenia: Imprecision or distractibility? Archives of General Psychiatry 57:1149-55. [DCJ, aWAP]

Raffone, A., Murre, J. M. J. \& Wolters, G. (in preparation) Cortical mechanisms for maintenance and control in visual working memory. [AR]

Raffone, A. \& Wolters, G. (2001) A cortical mechanism for binding in visual working memory. Journal of Cognitive Neuroscience 13:766-85. [aWAP, AR]

Raffone, A., Wolters, G. \& Murre J. M. J. (2001) A neurophysiological account of working memory limited capacity: Within-chunk integration and betweenitem segregation. Behavioral and Brain Sciences 24:139-41. [AR]

Raizada, R. D. S. \& Grossberg, S. (2001) Context-sensitive binding by the laminar circuits of V1 and V2: A unified model of perceptual grouping, attention, and orientation contrast. Visual Cognition 8:431-46. [rSMS]

(2003) Towards a theory of the laminar architecture of cerebral cortex: Computational clues from the visual system. Cerebral Cortex 13:100-13. $[\mathrm{SG}]$

Read, J., Perry, B. D., Moskowitz, A. \& Connolly, J. (2001). The contribution of early traumatic events to schizophrenia in some patients: A traumagenic neurodevelopmental model. Psychiatry 64:319-45. [rSMS]

Reeke, G. N. (2001) Modeling criteria: Not just for robots. Behavioral and Brain Sciences 24:1074-75. [rSMS]

Rennie, C. J., Wright, J. J. \& Robinson, P. A. (2000) Mechanisms of cortical electrical activity amd emergence of gamma rhythm. Journal of Theoretical Biology 205:17-35. [LMW]

Reuter, L. E., Fornal, C. A. \& Jacobs, B. L. (1997) A critical review of 5-HT brain microdialysis and behavior. Reviews in the Neurosciences 8:117-37. [RDO]

Reynolds, J. H. \& Desimone, R. (2003) Interacting roles of attention and visual salience in V4. Neuron 37:853-63. [rSMS]

Rief, W. (1991) Visual perceptual organization in schizophrenic patients. British Journal of Clinical Psychology 30 (Pt 4):359-66. [YC]

Riley, E. M., McGovern, D., Mockler, D. C, Doku, V. C. K., OiCeallaigh, S. \& Fannon, D. (2000) Neuropsychological functioning in first-episode psychosis: Evidence of specific deficits. Schizophrenia Research 43:47-55. [aWAP]

Riscalla, L. (1980) Blindness and schizophrenia. Medical Hypotheses 6:1327-28. [GSS]

Robbins, T. W. (1990) The case of frontostriatal dysfunction in schizophrenia Schizophrenia Bulletin 16:391-402. [aWAP]

(1996) Dissociating executive functions of the prefrontal cortex. Philosophical Transactions of the Royal Society of London B 351:1463-71. [aWAP]

Rochester, S. R. (1978) Are language disorders in acute schizophrenics actually information processing problems? Journal of Psychiatric Research 14:275-83. [aWAP]

Roelfsema, P. R., Engel, A. K., König, P. \& Singer, W. (1997) Visuomotor integration is associated with zero time-lag synchronization among cortical areas. Nature 385:157-61. [aWAP]

Roelfsema, P. R., König, P., Engel, A. K., Sireteanu, R. \& Singer, W. (1994 Reduced synchronization in the visual cortex of cats with strabismic amblyopia. European Journal of Neuroscience 6:1645-55. [aWAP]

Roelfsema, P. R., Lamme, V. A. F. \& Spekreijse, H. (2000) The implementation of visual routines. Vision Research 40:1385-411. [PRR]

Roelfsema, P. R., Lamme, V. A. F., Spekreijse, H. \& Bosch, H. (2002) Figureground segregation in a recurrent network architecture. Journal of Cognitive Neuroscience 14:525-37. [PRR]

Rosenbaum, G., Cohen, B. D., Luby, E. D., Gottlieb, J. S. \& Yelen, D. (1959) Comparison of sernyl with other drugs. Archives of General Psychiatry 1:65156. [aWAP]

Rourke, B. P. (1982) Central processing deficiencies in children: Toward a developmental neuropsychological model. Journal of Clinical Neuropsychology 4:1-18. [aWAP]

Roy, S. A., Dear S. P. \& Alloway K. D. (2001) Long-range cortical synchronization without concomitant oscillations in the somatosensory system of anesthetized cats. Journal of Neuroscience 21(5):1795-808. [MAT]

Rudy, J. W. \& Sutherland, R. J. (1989) The hippocampal formation is necessary for rats to learn and remember configural discriminations. Behavioral Brain Research 34:97-109. [JPG]

Rugg, M. D., Furda, J. \& Lorist, M. (1988) The effects of the task on the modulation of event-related potentials by word repetition. Psychophysiology 25:55-63. [DT]

Sah, P. \& Nicoll, R. A. (1991) Mechanisms underlying potentiation of synaptic transmission in rat anterior cingulate cortex in vitro. The Journal of Physiology 433:615-30. [GSS]

Salin, P. \& Bullier, J. (1995) Corticocortical connections in the visual system: Structure and function. Physiological Reviews 75:107-54. [aWAP]

Salisbury, D. F., Shenton, M. E., Griggs, C. B., Bonner-Jackson, A. \& McCarley, R. W. (2002) Mismatch negativity in chronic schizophrenia and first-episode schizophrenia. Archives of General Psychiatry 59:686-94. [rSMS]

Salt, T. E. \& Eaton, S. A. (1996) Functions of ionotropic and metabotropic glutamate receptors in sensory transmission in the mammalian thalamus. Progress in Neurobiology 48:55-72. [aWAP]

Sanders, G. S., Gallup, G. G., Heinsen, H., Hof, P. R. \& Schmitz, C. (2002) Cognitive deficits, schizophrenia, and the anterior cingulate cortex. Trends in Cognitive Sciences 6:190-92. [GSS]

Sandler, A. \& Hobson, R. (2001) On engaging with people in early childhood: The case of congenital blindness. Clinical Child Psychology and Psychiatry 6:20522. $[\mathrm{GSS}]$

Sanislow, C. A. \& Carson, R. C. (2001) Schizophrenia: A critical examination. In: 
Comprehenive handbook of psychopathology, ed. P. B. Sutker \& H. E. Adams. Kluwer Academic/Plenum. [JPG]

Sarfati, Y. \& Hardy-Bayle, M. (1999) How do people with schizophrenia explain the behaviour of others? A study of theory of mind and its relationship to thought and speech disorganization in schizophrenia. Psychological Medicine 29:613-20. [DL]

Sarfati, Y., Hardy-Bayle, M. C., Besche, C. \& Wildlöcher, D. (1997) Attribution of intentions to others in people with schizophrenia: A non-verbal exploration with comic strips. Schizophrenia Research 25:199-209. [DL]

Sarfati, Y., Hardy-Bayle, M. C., Nadel, J., Chevalier, J.-F. \& Widlöcher, D. (1997) Attribution of mental states to others by schizophrenic patients. Cognitive Neuropsychiatry 2:1-17. [rSMS]

Sass, L. (1992) Madness and modernism: Insanity in the light of modern art, literature, and thought. Basic Books. [rSMS]

(2000) Schizophrenia, self-experience, and the so-called "negative symptoms" (Reflections on hyperreflexivity). In: Exploring the self: Philosophical and psychopathological perspectives on self-experience, ed. D. Zahavi. John Benjamins. [LAS]

Sass, L. (in press). Schizophrenia: A disturbance of the thematic field. In: Gurwitsch's relevance for cognitive science, ed. L. Embree. Kluwer. [LAS]

Sass, L. \& Parnas, J. (in press a) Explaining schizophrenia: The relevance of phenomenology. In: The philosophical understanding of schizophrenia, ed. M. Chung, W. Fulford \& G. Graham. Oxford University Press. [LAS]

(in press b). Schizophrenia, consciousness, and the self. Schizophrenia Bulletin. [LAS]

Schenkel, L. S., Meredith, C., Purcell, L., Ritchie, A. J. \& Spaulding, W. D. (2001) Cognitive correlates of perceptual context processing in schizophrenia. Poster session presented at the meeting of the Society for Research in Psychopathology, Madison, WI, September 2001. [rSMS]

Schenkel, L. S. \& Spaulding, W. D. (2002) Social inference in schizophrenia: Relationships with childhood functioning and later cognitive impairment. Poster session presented at the meeting of the Society for Research in Psychopathology, San Francisco, CA, September 2002. [rSMS]

Schmidt, K. E., Löwel, S., Goebel, R. \& Singer, W. (1997) The perceptual grouping criterion of colinearity is reflected by anisotropies of connections in primary visual cortex. European Journal of Neuroscience 9:1083-89. [aWAP]

Schonauer, K., Achtergarde, D., Suslow, T. \& Michael, N. (1999) Comorbidity of schizophrenia and prelingual deafness: Its impact on social network structures. Social Psychiatry and Psychiatric Epidemiology 34:526-32. [GSS]

Schroeder, C. E. (1995) Defining the neural bases of visual selective attention Conceptual and empirical issues. International Journal of Neuroscience 80:65-78. [DCJ]

Selemon, L. D. \& Goldman-Rakic, P. S. (1999) The reduced neuropil hypothesis: A circuit based model of schizophrenia. Biological Psychiatry 45:17-25. [REH]

Selemon, L. D., Rajkowski, G. \& Goldman-Rakic, P. S. (1995) Abnormally high neuronal density in schizophrenic cortex: A morphometric analysis of prefrontal area 9 and occipital cortex area 17. Archives of General Psychiatry 52:805-18. [REH]

Selverston, A. I. (2001) Biomimetic robots and biology. Behavioral and Brain Sciences 24(6):1077. [rSMS]

Serper, M., Alpert, M., Richardson, N. A. \& Dickson, S. (1995) Clinical effects of recent cocaine use in patients with acute schizophrenia. American Journal of Psychiatry 152:1464-69. [MA]

Serper, M., Alpert, M. \& Trujillo, M. (1996) Recent cocaine use decreases negative signs in acute schizophrenia. Biological Psychiatry 39:816-18. [MA]

Servan-Schreiber, D., Cohen, J. D. \& Steingard, S. (1996) Schizophrenic deficits in the processing of context: A test of a theoretical model. Archives of General Psychiatry 53:1105-12. [DMB, AWM, aWAP, rSMS]

Shakow, D. (1962) Segmental set: A theory of formal psychological deficit in schizophrenia. Archives of General Psychiatry 6:1-17. [aWAP]

Shallice, T. (1988) From neuropsychology to mental structure. Cambridge University Press. [DRH, aWAP]

Shallice, T. \& Burgess, P. (1996) The domain of supervisory processes and temporal organization of behaviour. Philosophical Transactions of the Royal Society of London B 351:1405-11. [aWAP]

Shallice, T., Fletcher, P., Frith, C. D. Grasby, P. M., Frackowiak, R. S. J. \& Dolan, R. J. (1994) Brain regions associated with acquisition and retrieval of verbal episodic memory. Nature 368:633-35. [aWAP]

Sheer, D. E. (1984) Focused arousal, 40-Hz EEG, and dysfunction. In: Selfregulation of the brain and behavior, ed. T. Elbert, B. Rockstroh, W. Lutzenberger \& N. Birbaumer. Springer-Verlag. [LMW]

Shim, S. S. (2002) Is NMDA receptor hypofunction in schizophrenia associated with a primary hyperglutamatergic state? Archives of General Psychiatry 59:466-67. [RDO]

Siegel, S. J.,Brose, N.,Jansenn, W. G., Gasic, G. P., Jahn, R. \& Heinemann, S. F. (1994) Regional, cellular,and ultrastructural distribution of N-methyl-Daspartate receptor subunit 1 in monkey hippocampus. Proceedings of the National Academy of Science USA 91:564-68. [aWAP]
Silverstein, S. M. (1988) A study of religious conversion in North America. Genetic, Social, and General Psychology Monographs 114:261-305. [aWAP]

Silverstein, S. M., Baksi, S., Chapman, R. M. \& Nowlis, G. (1998a) Perceptual organization of configural and nonconfigural visual patterns in schizophrenia: Effects of repeated exposure. Cognitive Neuropsychiatry 3:209-23. [aWAP, rSMS]

Silverstein, S. M., Hatashita-Wong, M. \& Bloch, A. (2002) A second chance for people with "treatment-refractory" psychosis. Psychiatric Services 53:480. [rSMS]

Silverstein, S. M., Knight, R. A., Schwarzkopf, S. B., West, L. L., Osborn, L. M. \& Kamin, D. (1996a) Stimulus configuration and context effects in perceptual organization in schizophrenia. Journal of Abnormal Psychology 105:410-20. [aWAP, rSMS]

Silverstein, S. M., Kovács, I., Corry, R. \& Valone, C. (2000) Perceptual organization, the disorganization syndrome, and context processing in schizophrenia. Schizophrenia Research 43:11-20. [YC, aWAP, rSMS]

Silverstein, S. M., Light, G. A. \& Palumbo, D. R. (1998b) The sustained attention test: A measure of cognitive dysfunction. Computers in Human Behavior 14:463-75. [aWAP, rSMS]

Silverstein, S. M., Matteson, S. \& Knight, R. A. (1996b) Reduced top-down influence in auditory perceptual organization in schizophrenia. Journal of Abnormal Psychology 105:663-67. [aWAP, rSMS]

Silverstein, S. M., Menditto, A. A. \& Stuve, P. (2001) Shaping attention span: An operant conditioning procedure to improve neurocognition and functioning in schizophrenia. Schizophrenia Bulletin 27:247-57. [rSMS]

Silverstein, S. M., Osborn, L. M. \& Palumbo, D. R. (1998c) Rey-Osterreith Complex Figure Test performance in acute, chronic, and outpatient schizophrenia patients. Journal of Clinical Psychology 54:1-10. [aWAP]

Silverstein, S. M., Osborn, L. M., West, L. L. \& Knight, R. A. (1998d) Perceptual organization in schizophrenia: Evidence for intact processing of configural patterns. Cognitive Neuropsychiatry 3:225-35. [aWAP]

Silverstein, S. M. \& Palumbo, D. R. (1995). Nonverbal perceptual organization output disability and schizophrenia spectrum symptomatology. Psychiatry 58:66-81. [aWAP]

Silverstein, S. M. \& Schenkel, L. S. (1997) Schizophrenia as a model of contextdeficient cortical computation. Behavioral and Brain Sciences 20:696-97. [aWAP]

Silverstein, S. M., Schenkel, L. S. \& Valone, C. (1998e) Cognitive deficits and psychiatric rehabilitation outcomes in schizophrenia. Psychiatric Quarterly 69:169-91. [aWAP]

Singer, W. (1994) Coherence as an organizing principle of cortical functions. International Review of Neurobiology 37:153-83. [aWAP]

(1995) Development and plasticity of cortical processing architectures. Science 270:758-64. [aWAP]

(1999) Neuronal synchrony, a versatile code for the definition of relations? Neuron 24:49-64. [aWAP]

Singer, W. \& Gray, C. M. (1995) Visual feature integration and the temporal correlation hypothesis. Annual Review of Neuroscience 18:555-86. [aWAP, PPR]

Sitnikova, T., Salisbury, D. F., Kuperberg, G. \& Holcomb, P. I. (2002) Electrophysiological insights into language processing in schizophrenia. Psychophysiology 39:851-60. [DT]

Smith, R. E., Haroutunian, V., David, L. \& Meador-Woodruff, J. H. (2001) Expression of excitatory amino acid transporter transcripts in the thalamus of subjects with schizophrenia. American Journal of Psychiatry 158:1393-99. [rSMS]

Solovay, M. R., Shenton, M. E., Gasparetti, C., Coleman, M., Kestnbaum, E., Carpenter, J. T. \& Holzman, P. S. (1986) Scoring manual for the Thought Disorder Index. Schizophrenia Bulletin 12:483-96. [aWAP]

Solovay, M. R., Shenton, M. E. \& Holzman, P. S. (1987) Comparative studies of thought disorders. I. Mania and schizophrenia. Archives of General Psychiatry 44:13-20. [rSMS]

Spaulding, W. D., Reed, D., Sullivan, M., Richardson, C. \& Weiler, M. (1999) Effects of cognitive treatment in psychiatric rehabilitation. Schizophrenia Bulletin 25:657-76. [rSMS]

Spitzer, M. (1997) A cognitive neuroscience view of schizophrenic thought disorder. Schizophrenia Bulletin 23:29-50. [DRH]

Spitzer, M., Beuckers, J., Beyer, S., Maier, S. \& Hermle, L. (1994) Contextual insensitivity in thought-disordered schizophrenic patients: Evidence from pauses in spontaneous speech. Language and Speech 37:171-85. [aWAP]

Spitzer, M., Braun, U., Hermle, L. \& Maier, S. (1993) Associative semantic network dysfunction in thought-disordered schizophrenia patients: Direct evidence from indirect semantic priming. Biological Psychiatry 34:864-77. [aWAP]

Sporns, O., Gally, J. A., Reeke, G. N. \& Edelman, G. M. (1989) Reentrant signaling among simulated neuronal groups leads to coherency in their oscillatory activity. Proceedings of the National Academy of Science USA 86:7265-69. [aWAP, rSMS]

Stoet, G. \& Hommel, B. (1999) Action planning and the temporal binding of 
response codes. Journal of Experimental Psychology: Human Perception and Performance 25:1625-40. [aWAP]

Stone, J. V. \& Kotter, R. (2002) Making connections about brain connectivity. Trends in Cognitive Sciences 6:327-28. [SLB]

Strandburg, R. J., Marsh, J. T., Brown, W. S., Asarnow, R. F., Guthrie, D., Harper, R., Ylee, C. M. \& Nuechterlein, K. H. (1997) Event related potential correlates of linguistic information processing in schizophrenics. Biological Psychiatry 42:596-608. [DRH]

Stratta, P., Daneluzzo, E., Bustini, M., Casacchia, M. \& Rossi, A. (1998) Schizophrenic deficits in the processing of context. Archives of General Psychiatry 55:186-87. [DMB]

Straube, E. R. \& Oades, R. D. (1992) Schizophrenia: Empirical research and findings. Academic Press. [RDO]

Strelets, V., Faber, P. L., Golicova, J., Novototsky-Vlasov, V., Koenig, T., Gianotti, L. R. R., Gruzelier, J. H. \& Lehman, D. (under review) Chronic schizophrenics with positive symptomatology have shortened EEG microstate durations. Clinical Neurophysiology. [VS]

Strelets V. B., Novototsky-Vlasov V. Y. \& Golikova J. V. (2002) Cortical connectivity in high frequency beta-rhythm in schizophrenics with positive and negative symptoms. International Journal of Psychophysiology 44:101-15. [VS]

Sullivan, H. S. (1956) The schizophrenic dynamism: A tripartite view. In: H. S. Sullivan, Clinical studies in psychiatry. W. W. Norton. [rSMS]

Sutherland, R. J. \& Rudy, J. W. (1989) Configural association theory: The role of the hippocampal formation in learning, memory, and amnesia. Psychobiology 17:129-44. [JPG]

Svensson, T. H. (2000) Dysfunctional brain dopamine systems induced by psychotomimetic NMDA-receptor antagonists and the effects of antipsychotic drugs. Brain Research - Brain Research Reviews 31:320-29. [MAT]

Tagamets, M. A. \& Horwitz, B. (1998) Integrating electrophysiological and anatomical experimental data to create a large-scale model that simulates a delayed matchto-sample human brain imaging study. Cerebral Cortex 8:310-20. [MAT]

Talamini, L. M., Meeter, M., Murre, J. M. J., Elvevåg, B. \& Goldberg, T. E. (in preparation) Integration of parallel input streams in parahippocampal model circuits; implications for schizophrenia. [LMT]

Tallent, K. A. \& Gooding, D. C. (2000) Working Memory and Wisconsin Card Sorting Test performance in schizotypic individuals: A replication and extension. Psychiatry Research 89:161-70. [DCG]

Tallon-Baudry, C., \& Bertrand, O. (1999) Oscillatory gamma activity in humans and its role in object representation. Trends in the Cognitive Sciences 3:15162. [aWAP]

Tamminga, C. A. (1998) Schizophrenia and glutamatergic transmission. Critical Review of Neurobiology 12:21-36. [aWAP, MAT]

Tamminga, C. A., Holcomb, H. H., Gao, X. M. \& Lahti, A. C. (1995) Glutamate pharmacology and the treatment of schizophrenia: Current status and future directions. International Clinical Psychopharmacology 10 (Suppl. 3):29-37. [aWAP]

Tarnowski, K. J., Prinz, R. J. \& Nay, S. M. (1986). Comparative analysis of attentional deficits in hyperactive and learning-disabled children. Journal of Abnormal Psychology 95:341-45. [aWAP]

Tellegen, A. \& Atkinson, G. (1974) Openness to absorbing and self-altering experiences ('absorption'), a trait related to hypnotic susceptibility. Journal of Abnormal Psychology 83:268-277. [aWAP]

Tendolkar, I., Ruhrman, S., Brockhaus, A., Pukrop, R. \& Klosterkotter, J. (2002) Remembering or knowing: Electrophysiological evidence for an episodic memory deficit in schizophrenia. Psychological Medicine 32:1261-71. [rSMS]

Thagard, P. (2000) Coherence in thought and action. MIT Press. [SLB]

Tien, A. Y. (1999) Deficits of information management associated with schizophrenia: Awareness and associated integrative cognitive functions. Archives of General Psychiatry 56:637-38. [rSMS]

Tiitinen, H., Sinkkonen, J., Reinikainen, K., Alho, K., Lavikainen, J. \& Näätänen R. (1993) Selective attention enhances the auditory $40-\mathrm{Hz}$ transient response in humans. Nature 364:59-60. [aWAP]

Titone, D., Levy, D. \& Holzman, P. S. (2000) Contextual insensitivity in schizophrenic language processing: Evidence from lexical ambiguity. Journal of Abnormal Psychology 109:761-67. [DT]

Titone, D., Holzman, P. S. \& Levy, D. L. (2002) Idiom processing in schizophrenia: Literal implausibility saves the day for idiom priming. Journal of Abnormal Psychology 111:313-20. [DT]

Tononi, G. \& Edelman, G. M. (2000) Schizophrenia and the mechanisms of conscious integration. Brain Research Reviews 31:391-400. [aWAP]

Tononi, G., McIntosh, A. R., Russell, D. P. \& Edelman, G. M. (1998a) Functional clustering: Identifying strongly interactive brain regions in neuroimaging data. Neuroimage 7:133-49. [aWAP]

Tononi, G., Sporns, O. \& Edelman, G. M. (1994) A measure for brain complexity: Relating functional segregation and integration in the nervous system. Proceedings of the National Academy of Science USA 91:5033-37. [aWAP]

(1996) A measure for selective matching of signals by the brain. Proceedings of the National Academy of Science USA 93:3422-27. [aWAP] (1998b) Complexity and coherency: Integrating information in the brain. Trends in the Cognitive Sciences 2:474-84. [aWAP]

Tost, H., Wolf, I. G., Ruf, M., Henn, F. A. \& Braus, D. F. (2001) Disturbed visual information processing in neuroleptic-naive schizophrenia patients: A fMRI study. Schizophrenia Research 49:187. [aWAP]

Treisman, A. (1999) Solutions to the binding problem: progress through controversy and convergence. Neuron 24:105-10. [aWAP]

Treue, S. \& Tujillo, J. C. M. (1999) Feature-based attention influences motion processing gain in macaque visual cortex. Nature 399:575-79. [aWAP]

Tsai, G., Passani, L. A., Slusher, B. S., Carter, R., Baer, L., Kleinman, J. E. \& Coyle, J. T. (1995) Abnormal excitatory neurotransmitter metabolism in schizophrenic brains. Archives of General Psychiatry 52:829-36. [rSMS]

Turvey, M. T. (1977) Preliminaries to a theory of action with reference to vision. In Perceiving, acting and knowing, ed. R. Shaw \& J. Bransford. Erlbaum. [aWAP]

Uhlhaas, P. (2003) Gestalt perception in schizophrenia spectrum disorders. Doctoral dissertation, University of Stirling, UK. [rSMS]

Uhlhaas, P. J., Silverstein, S. M., Phillips, W. A. \& Lovell, P. G. (submitted) Evidence for impaired visual context processing in schizotypy with thought disorder. [rSMS]

Umbricht, D., Schmid, L., Koller, R., Vollenweider, F. X., Hell, D. \& Javitt, D. C. (2000) Ketamine-induced deficits in auditory and visual context-dependent processing in healthy volunteers. Implications for models of cognitive deficits in schizophrenia. Archives of General Psychiatry 57:1139-47. [DCJ, RDO, aWAP, GSS, rSMS]

Umbricht, D., Vollenweider, F. X., Schmid, L., Grubel, C., Skrabo, A., Huber, T. \& Koller, R. (2003) Effects of the 5-HT(2A) agonist psilocybin on mismatch negativity generation and AX-continuous performance task: Implications for the neuropharmacology of cognitive deficits in schizophrenia. Neuropsychopharmacology 28:170-181. [rSMS]

Van Berckel, B. N., Hijman, R., van der Linden, J. A., Westenberg, H. G., van Ree, J. M. \& Kahn, R. S. (1996) Efficacy and tolerance of D-cycloserine in drugfree schizophrenic patients. Biological Psychiatry 40:1298-300. [MAT]

Van Berckel, B. N., Oranje, B., van Ree, J. M., Verbaten, M. N. \& Kahn, R. S. (1998) The effects of low dose ketamine on sensory gating, neuroendocrine secretion and behavior in healthy human subjects. Psychopharmacology (Berlin) 137:271-81. [MAK, MEP, rSMS]

Van der Velde, F. \& de Kamps, M. (2001) From knowing what to knowing where: Modeling object-based attention with feedback disinhibition of activation. Journal of Cognitive Neuroscience 13:479-91. [PRR]

Van Hoof, J. J. M. (2002) The abnormal development of drive and guidance mechanisms in the brain: The pathogenesis of schizophrenia, Acta Neuropsychiatrica 14:134-46. [HPB]

Varela, F. (1995) Resonant cell assemblies: A new approach to cognitive functions and neuronal synchrony. Biological Research 28:81-95. [rSMS]

Varela, F. J. (1996) Neurophenomenology; a methodological remedy for the hard problem. Journal of Consciousness Studies 3:330-49. [LAS]

Varela, F., Lachaux, J. P., Rodriguez, E. \& Martinerie, J. (2001) The brainweb: Phase synchronization and large-scale integration. Nature Reviews Neuroscience 2:229-39. [SLB]

Venables, P. (1964) Input dysfunction in schizophrenia. Progress in Experimental Personality Research 1:1-47. [MAK]

Vizuete, M. L., Venero, J. L., Vargas, C., Revuelta, M., Machado, A. \& Cano, J. (2001) Potential role of endogenous brain-derived neurotrophic factor in longterm neuronal reorganization of the superior colliculus after bilateral visual deprivation. Neurobiology of Disease 8:866-80. [GSS]

Vogt, B. A. \& Miller, M. W. (1983) Cortical connections between rat cingulate cortex and visual, motor, and postsubicular cortices. The Journal of Comparative Neurology 216:192-210. [GSS]

Vollenweider, F. X., Leenders, K. L., Oye, I., Hell, D. \& Angst, J. (1997a) Differential psychopathology and patterns of cerebral glucose utilization produced by $(\mathrm{S})$-ketamine and $(\mathrm{R})$-ketamine in healthy volunteers using positron emision tomography (Pet). European Neuropsychopharmacology 7:25-38. [aWAP]

Vollenweider, F. X., Leenders, K. L., Scharfetter, C., Antonini, A., Macguire, P., Missimer, J. \& Angst, J. (1997b) Metabolic hyperfrontality and psychopathology in the ketamine model of psychosis using positron emission tomography (Pet) and (F-18) fluorodexyglucose (Fdg). European Neuropsychopharmacology 7:9-24. [aWAP]

Von der Malsburg, C. \& Schneider, W. (1986) A neural cocktail-party processor. Biological Cybernetics 54:29-40. [aWAP]

Von Stein, A., Chiang, C. \& Konig, P. (2000) Top-down processing mediated by interareal synchronization. Proceedings of the National Academy of Sciences USA 97:14748-53. [rSMS]

Waberski, T. D., Kreitschmann-Andermahr, I., Kawohl, W., Darvas, F., Ryang, Y. Gobbele, R. \& Buchner, H. (2001) Spatio-temporal source imaging reveals subcomponents of the human auditory mismatch negativity in the cingulum and right inferior temporal gyrus. Neuroscience Letters 308:107-10. [GSS] 
References/Phillips \& Silverstein: Convergence of perspectives on cognitive coordination in schizophrenia

Walker, E. F. \& DiForio, D. (1996) Schizophrenia: A neural diathesis-stress model. Psychological Review 104:667-85. [DCG]

Waltz, J. A., Knowlton, B. J., Holyoak, K. J., Boone, K. B., Mishkin, F. S., Santos, M. D., Thomas, C. R. \& Miller, B. L. (1999) A system for relational reasoning in human prefrontal cortex. Psychological Science 10(2):119-25. [DL]

Wang, X. J. (1999) Synaptic basis of cortical persisent actyivity; the importance of NMDA receptors to working memory. Journal of Neuroscience 19:9587-603. [aWAP]

Warren, D. (1994) Blindness and children: An individual differences approach. Cambridge University Press. [GSS]

Watanabe, M., Inoue, Y., Sakimura, K. \& Mishina, M. (1992) Developmental changes in distribution of NMDA receptor channel subunit mRNAs. NeuroReport 3:1138-40. [aWAP]

Watt, R. J. \& Phillips, W. A. (2000) The function of dynamic grouping in vision. Trends in Cognitive Sciences 4:447-454. [aWAP, rSMS]

Weiler, M. A., Thaker, G. K., Lahti, A. C. \& Tamminga, C. A. (2000). Ketamine effects on eye movements. Neuropsychopharmacology 23:645-53. [aWAP]

Whittington, M. A., Stanford, I. M., Colling, S. B., Jefferys, J. G. \& Traub, R. D. (1997) Spatiotemporal patterns of gamma frequency oscillations tetanically induced in the rat hippocampal slice. Journal of Physiology 502:591-607. [aWAP]

Whittington, M. A., Traub, R. D. \& Jefferys, J. G. R. (1995) Synchronized oscillations in interneuron networks driven by metabotropic glutamate receptor activation. Nature 373:612-15. [aWAP]

Winter, J. C., Fiorella, D. J., Timinieri, D. M., Filipink, R. A., Helsley, S. E. \& Rabin, R. A. (1999) Serotonergic receptor subtypes and hallucinogen-induced stimulus control. Pharmacology, Biochemistry and Behavior 64:283-93. [RDO]

Winterer, G., Egan, M. F., Radler, T., Hyde, T., Coppola, R. \& Weinberger, D. R. (2001) An association between reduced interhemispheric EEG coherence in the temporal lobe and genetic risk for schizophrenia. Schizophrenia Research 49:129-43. [rSMS]

Wooley, D. W. \& Shaw, W. (1954) A biochemical and pharmacological suggestion about certain mental disorders. Proceedings of the National Academy of Sciences USA 40:228-31. [rSMS]

Wörgötter, F. \& Eysel, U. F. (2000) Context, state and the receptive fields of striatal cortex cells. Trends in the Neurosciences 23:497-503. [aWAP]

Wright, J. J., Robinson, P. A., Rennie, C. J., Gordon, E., Bourke, P. D., Chapman, C. J., Hawthorn, N., Lees, G. J. \& Alexander, D. (2001) Toward an integrated continuum model of cerebral dynamics: The cerebral rhythms, synchronous oscillation and cortical stability. Biosystems 63:71-88. [aWAP]

Xing J. \& Heeger, D. J. (2000) Center-surround interactions in foveal and peripheral vision. Vision Research 40(22):3065-72. [YC]

Yamaguchi, K., Nabeshima, T., Ishikawa, K., Yoshida, S. \& Kameyama, T. (1987) Phencylidine-induced head-weaving and head-twitch through interaction with 5- $\mathrm{HT}_{2 \mathrm{~A}}$ receptors in reserpinized rats. Neuropharmacology 26:1489-97. [rSMS]

Yung, A. R., Phillips, L. J., Yuen, H. P., Francey, S. M., McFarlane, C. A., Hallgren, M. \& McGorry, P. D. (2003) Psychosis prediction: 12-month follow up of a high-risk ("prodromal") group. Schizophrenia Research 60:21-32. [rSMS]

Zipser, K., Lamme, V. A. F. \& Schiller, P. H. (1996) Contextual modulation in primary visual cortex. Journal of Neuroscience 16:7376-89. [aWAP, PPR, rSMS]

Zukin, S. R., Fitz-Syage, M. L., Nichtenhauser, R. \& Zukin, R. S. (1983) Specific binding of $[3 \mathrm{H}]$ phencyclidine in rat central nervous tissue: Further characterization and technical considerations. Brain Research 258:277-84. [rSMS]

Zukin, S. R. \& Zukin, R. S. (1979) Specific $[3 \mathrm{H}]$ phencyclidine binding in rat central nervous system. Proceedings of the National Academy of Sciences USA 76:5372-76. [rSMS] 